University of Tennessee Health Science Center

UTHSC Digital Commons

\title{
Discoveries of Targets and Novel Agents for the Treatment of Ischemic Retinopathy and Neovascular Disease
}

Jordan Javad Toutounchian

University of Tennessee Health Science Center

Follow this and additional works at: https://dc.uthsc.edu/dissertations

Part of the Pharmaceutics and Drug Design Commons

\section{Recommended Citation}

Toutounchian, Jordan Javad (http://orcid.org/0000-0003-3075-7680), "Discoveries of Targets and Novel Agents for the Treatment of Ischemic Retinopathy and Neovascular Disease" (2016). Theses and Dissertations (ETD). Paper 405. http://dx.doi.org/10.21007/etd.cghs.2016.0409. 


\title{
Discoveries of Targets and Novel Agents for the Treatment of Ischemic Retinopathy and Neovascular Disease
}

\begin{abstract}
Diabetic retinopathy (DR) and age-related macular degeneration (AMD) are among the most common causes of blindness in adults. Vision loss can occur during the advanced stages of DR and AMD as a consequence of unregulated and dysfunctional growth of new blood vessels, or neovascularization (NV) in the retina or choroid. NV can also be triggered by numerous other ocular insults and diseases including radiation retinopathy (RR) and retinal vein occlusion. These latter cases are generally less common but, like DR and AMD, they are characterized by an initial injury, chronic inflammation, and ischemia which perpetuates episodes of retinal neovascularization (RNV).

Current targets for RNV include vascular endothelial growth factor, VEGF which is achieved through antiVEGF protein therapeutics aimed at sequestering the growth factor and preventing the activation of its receptor. However, prospective studies show that anti-VEGF resistance has become a major clinical concern in patients receiving long-term therapy. Thus, targeting downstream signaling proteins linked to pathological RNV represents an alternative or adjunctive approach to approved anti-VEGF treatments, which may provide better patient outcomes through enhanced efficacy of antiangiogenic therapy.

Our first goal was to understand how RNV progresses from early stage injury to proliferative ischemic retinopathy, in order to justify protein targets for drug discovery. We first began with an investigation into the causality of radiation injury itself to identify mechanisms of radiation sensitivity and/or resistance in the genetically diverse, murine BXD strains using a total-body irradiation (TBI) model. Our studies suggested mean survival time (MST) over 30 days may in fact be related to genetic variation in genes associated with endothelial progenitor cells (EPC) localization, wound healing, and focal adhesion (FA) dynamics involving both the hematopoietic and gastrointestinal systems. We targeted these mechanisms of tissue repair by blocking the homing of hematopoietic-derived cells to sites of irradiation (IR) injury which proved fatal to mice treated with an integrin-paxillin inhibitor, 6-B345TTQ. In a physiological flowbased assay, we inhibited circulating leukocytes from interacting with an inflamed endothelium, in vitro. These results suggested that the reparative/inflammatory angiogenic response triggered by radiation could be blocked by targeting FA signaling, a central process of RNV progression in ischemic retinopathies.
\end{abstract}

Findings in BXD studies linked tissue reparative processes involving ischemia- induced angiogenesis with mortality. We hypothesized that by targeting early injury in retinal endothelial cells (REC), we could prevent late-stage RNV. Thus, we first explored how RECs respond to radiation injury at levels high enough to cause significant vision impairments in RR. Previously identified radioprotectant, KZ-41, was used in these studies to ameliorate IR-induced injury to RECs through decreased inflammatory stress kinase activation, cell death, and subsequent IR-induced proliferation, in vitro. FA activation through paxillin was found to be a crucial mechanism by which KZ-41 inhibited ischemia-induced RNV in the murine oxygen-induced retinopathy (OIR) model.

Targeting stress kinase activation of FA signaling post-IR injury served as a way to prevent the pathological progression of RNV, in vivo. However, it is difficult to predict when or how to treat the inflammation early in ischemic retinopathy, especially in chronic conditions such as diabetes, when the injury has already occurred. Therefore, we sought to target the common focal point of ischemic disease by focusing on drivers of late stage RNV, the focal adhesion signaling complex. Using VEGF as the driver of in vitro angiogenesis, we explored growth factor-induced FA signaling in RECs to validate target proteins Src, focal adhesion kinase (FAK), and paxillin as crucial to RNV progression. Our work helped to identify a novel paxillin modulator, JP-153 which afforded excellent antiangiogenic activity, in vitro. JP-153 
achieved potent inhibition of RNV in the OIR model through topical application by disrupting paxillin activation. Together, these data suggested paxillin is a key driver of RNV and may serve as a viable target for the treatment of neovascular eye disease.

In Chapter 6, we characterized the pharmacokinetic profile of JP-153 with regard to its absorption, distribution, metabolism, and elimination (ADME) after both oral and intravenous administration. We found that JP-153 exhibited rapid metabolism in rats with an oral bioavailability of approximately $30 \%$. During these studies, we successfully developed a sensitive and selective analytical method using mass spectrometry in order to detect JP-153 concentrations in rat plasma. JP-153 possessed a relatively rapid clearance profile, which is an ideal characteristic for ocular therapeutics. Lower systemic exposures decrease the risk of cardiovascular side effects, a common concern with antiangiogenic therapies. Though, further work to characterize its ocular pharmacokinetic profile is needed to identify the proper dosing regimen in future studies. Thus, these data herein have served as a basis for further development of JP-153 series analogs, used either as a topical or systemic therapeutic for in vivo efficacy studies and pre-clinical work.

In conclusion, our work has successfully provided rationales for new drug targets and clinically relevant pharmacological agents to halt RNV. The following chapters describe and discuss novel ways in which we target inflammatory signaling and protein-protein interactions related to FA protein paxillin to effectively stop angiogenesis in the retina. Importantly, targeting paxillin has much broader implications in treating angiogenesis in general, and work studying paxillin modulation in cancer cells represents interesting hypotheses for future work in our laboratory.

\section{Document Type}

Dissertation

Degree Name

Doctor of Philosophy (PhD)

Program

Pharmaceutical Sciences

Research Advisor

Charles R. Yates, Pharm.D./Ph.D.

\section{Keywords}

Angiogenesis, BXD, Focal Adhesion, Neovascularization, Paxillin, Retinopathy

\section{Subject Categories}

Medicine and Health Sciences | Pharmaceutics and Drug Design | Pharmacy and Pharmaceutical Sciences

\section{Comments}

Two year embargo expires August 2018. 


\title{
Discoveries of Targets and Novel Agents for the Treatment of Ischemic Retinopathy
} and Neovascular Disease

\author{
A Dissertation \\ Presented for \\ The Graduate Studies Council \\ The University of Tennessee \\ Health Science Center
}

\author{
In Partial Fulfillment \\ Of the Requirements for the Degree \\ Doctor of Philosophy \\ From The University of Tennessee
}

By

Jordan Javad Toutounchian

August 2016 
Portions of Chapter 4 (C) 2014 by Toutounchian, J.J., et al. . All other material (C) 2016 by Jordan J. Toutounchian. All rights reserved. 


\section{DEDICATION}

For my parents. 


\section{ACKNOWLEDGEMENTS}

The journey towards obtaining my $\mathrm{PhD}$ would not have been possible without the support and constant encouragement from many people. To my advisor and mentor, Dr. Ryan Yates, I owe you a tremendous amount of gratitude for the years you have put into my education at the University of Tennessee and the hours you have spent discussing everything and anything that came to mind. Your critical insight, clever retorts and hours of philosophical discussions have aided in my growth as a scientist, a leader and a future mentor. For those reasons, I will always be grateful. Thank you for your kindness and more importantly, your unfettered enthusiasm.

To my committee members, Dr. Bernd Meibohm, Dr. Duane Miller, Dr. Frank Park, Dr. David Rogers, and Dr. Jerome Baudry, I thank you for your involvement in my graduate years; it was a pleasure working with all of you. Your critical advice and wealth of knowledge has instilled in me the makings of a true pharmaceutical scientist.

I extend my sincerest appreciation to my colleagues, both past and present for their interest in me as a person and as a scientist. The years have gone by much too quickly, but the memories I now have will always remain the centerpiece of my graduate experience. Thank you for the wonderful moments and levity you have brought.

To my parents, Darlene and Javad, you are the reason I dedicate my life to academia and science. Through your endearing support and unwavering commitment to my education, I am able to think, breathe, rationalize, and love, but most importantly appreciate this life and the beauty it has to offer. All of my achievements, and all of my future endeavors will always be attributed to your eternal belief in my potential. And to the rest of my family, especially my sister Monica, your care and support has never gone unnoticed. All of your kindness, love and empathy has created my quiet temperament that has afforded me poise and grace in every aspect of my life. I hope that I have made you all proud.

Devlin, your thunderous disposition and beautiful nature shook the very foundation for which I stood and have resided all of these years. Your magnetism was/is insurmountable and our future is amazingly clear. The world awaits us, you and me, and on it we will make our mark. Thank you for always supporting me.

Funding for these projects was provided by the National Eye Institute's Vision Core Grant: PHS 3P30 and EY013080. Other support was kindly provided by an unrestricted grant from Department of Ophthalmology and from Research to Prevent Blindness. This work was also graciously supported by internal funds from the University of Tennessee College of Pharmacy and the University of Tennessee Research Foundation. And a special thanks to Mark Erickson from JirehDesign.com for illustrations used in Figure 1-2. 


\begin{abstract}
Diabetic retinopathy (DR) and age-related macular degeneration (AMD) are among the most common causes of blindness in adults. Vision loss can occur during the advanced stages of DR and AMD as a consequence of unregulated and dysfunctional growth of new blood vessels, or neovascularization (NV) in the retina or choroid. NV can also be triggered by numerous other ocular insults and diseases including radiation retinopathy $(\mathrm{RR})$ and retinal vein occlusion. These latter cases are generally less common but, like DR and AMD, they are characterized by an initial injury, chronic inflammation, and ischemia which perpetuates episodes of retinal neovascularization (RNV).

Current targets for RNV include vascular endothelial growth factor, VEGF which is achieved through anti-VEGF protein therapeutics aimed at sequestering the growth factor and preventing the activation of its receptor. However, prospective studies show that anti-VEGF resistance has become a major clinical concern in patients receiving longterm therapy. Thus, targeting downstream signaling proteins linked to pathological RNV represents an alternative or adjunctive approach to approved anti-VEGF treatments, which may provide better patient outcomes through enhanced efficacy of antiangiogenic therapy.
\end{abstract}

Our first goal was to understand how RNV progresses from early stage injury to proliferative ischemic retinopathy, in order to justify protein targets for drug discovery. We first began with an investigation into the causality of radiation injury itself to identify mechanisms of radiation sensitivity and/or resistance in the genetically diverse, murine BXD strains using a total-body irradiation (TBI) model. Our studies suggested mean survival time (MST) over 30 days may in fact be related to genetic variation in genes associated with endothelial progenitor cells (EPC) localization, wound healing, and focal adhesion (FA) dynamics involving both the hematopoietic and gastrointestinal systems. We targeted these mechanisms of tissue repair by blocking the homing of hematopoieticderived cells to sites of irradiation (IR) injury which proved fatal to mice treated with an integrin-paxillin inhibitor, 6-B345TTQ. In a physiological flow-based assay, we inhibited circulating leukocytes from interacting with an inflamed endothelium, in vitro. These results suggested that the reparative/inflammatory angiogenic response triggered by radiation could be blocked by targeting FA signaling, a central process of RNV progression in ischemic retinopathies.

Findings in BXD studies linked tissue reparative processes involving ischemiainduced angiogenesis with mortality. We hypothesized that by targeting early injury in retinal endothelial cells (REC), we could prevent late-stage RNV. Thus, we first explored how RECs respond to radiation injury at levels high enough to cause significant vision impairments in RR. Previously identified radioprotectant, KZ-41, was used in these studies to ameliorate IR-induced injury to RECs through decreased inflammatory stress kinase activation, cell death, and subsequent IR-induced proliferation, in vitro. FA activation through paxillin was found to be a crucial mechanism by which KZ-41 inhibited ischemia-induced RNV in the murine oxygen-induced retinopathy (OIR) model. 
Targeting stress kinase activation of FA signaling post-IR injury served as a way to prevent the pathological progression of $\mathrm{RNV}$, in vivo. However, it is difficult to predict when or how to treat the inflammation early in ischemic retinopathy, especially in chronic conditions such as diabetes, when the injury has already occurred. Therefore, we sought to target the common focal point of ischemic disease by focusing on drivers of late stage RNV, the focal adhesion signaling complex. Using VEGF as the driver of in vitro angiogenesis, we explored growth factor-induced FA signaling in RECs to validate target proteins Src, focal adhesion kinase (FAK), and paxillin as crucial to RNV progression. Our work helped to identify a novel paxillin modulator, JP-153 which afforded excellent antiangiogenic activity, in vitro. JP-153 achieved potent inhibition of RNV in the OIR model through topical application by disrupting paxillin activation. Together, these data suggested paxillin is a key driver of RNV and may serve as a viable target for the treatment of neovascular eye disease.

In Chapter 6, we characterized the pharmacokinetic profile of JP-153 with regard to its absorption, distribution, metabolism, and elimination (ADME) after both oral and intravenous administration. We found that JP-153 exhibited rapid metabolism in rats with an oral bioavailability of approximately $30 \%$. During these studies, we successfully developed a sensitive and selective analytical method using mass spectrometry in order to detect JP-153 concentrations in rat plasma. JP-153 possessed a relatively rapid clearance profile, which is an ideal characteristic for ocular therapeutics. Lower systemic exposures decrease the risk of cardiovascular side effects, a common concern with antiangiogenic therapies. Though, further work to characterize its ocular pharmacokinetic profile is needed to identify the proper dosing regimen in future studies. Thus, these data herein have served as a basis for further development of JP-153 series analogs, used either as a topical or systemic therapeutic for in vivo efficacy studies and pre-clinical work.

In conclusion, our work has successfully provided rationales for new drug targets and clinically relevant pharmacological agents to halt RNV. The following chapters describe and discuss novel ways in which we target inflammatory signaling and proteinprotein interactions related to FA protein paxillin to effectively stop angiogenesis in the retina. Importantly, targeting paxillin has much broader implications in treating angiogenesis in general, and work studying paxillin modulation in cancer cells represents interesting hypotheses for future work in our laboratory. 


\section{TABLE OF CONTENTS}

\section{CHAPTER 1. RETINAL NEOVASCULARIZATION - CURRENT STATE OF TREATMENT AND STRATEGIES FOR NOVEL DRUG TARGETS .........................1}

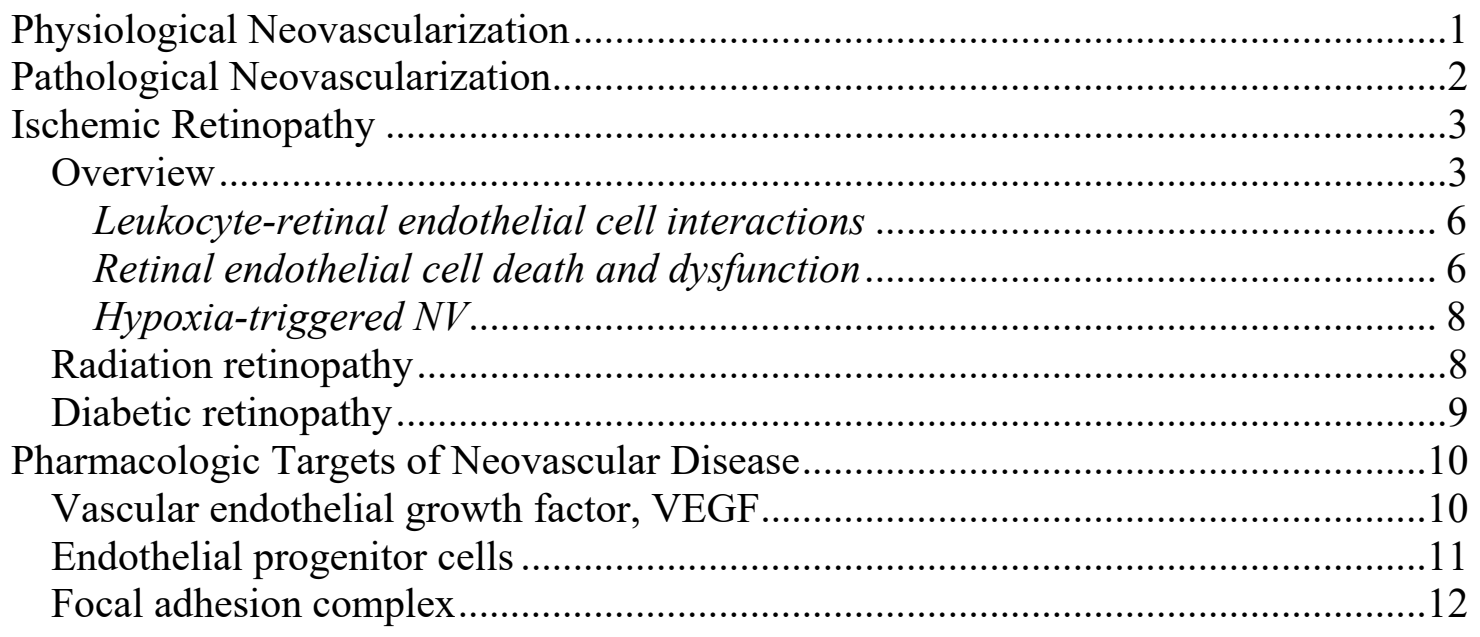

CHAPTER 2. CENTRAL HYPOTHESIS .......................................................................15

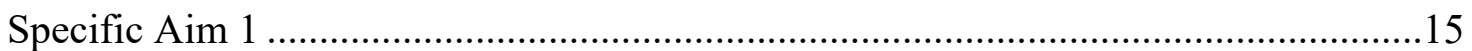

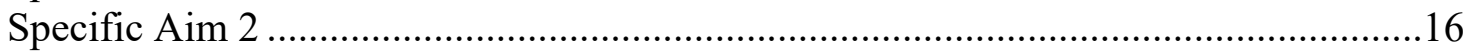

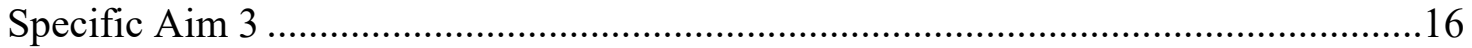

\section{CHAPTER 3. IDENTIFICATION OF CANDITATE GENES THAT CONFER RADIATION INJURY SUSCEPTIBILITY IN BXD MICE ....................................17}

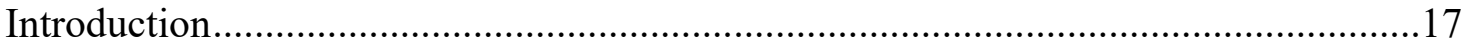

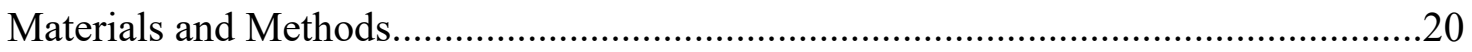

The murine total-body irradiation model ...............................................................20

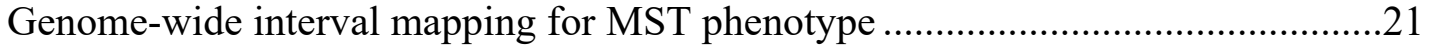

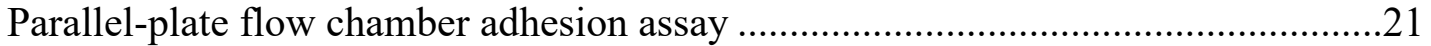

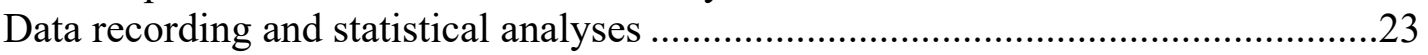

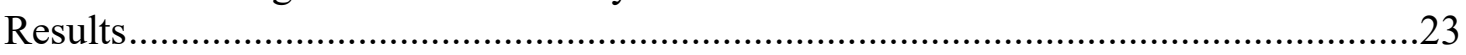

MST data shows differential susceptibility to TBI in BXD mice...........................23

Phenotypic correlations, QTL identification, and marker regression analysis ..........23

Affymetrix gene correlates reveal associations with genes of both hematopoietic

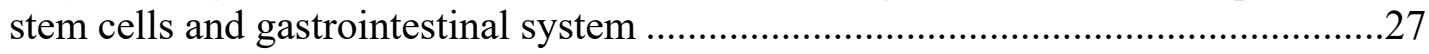

Targeting integrin-paxillin in C57BL/6J reduces survival following TBI ................32

6-B345TTQ prevents leukocyte adhesion to irradiated retinal endothelial cells.......32

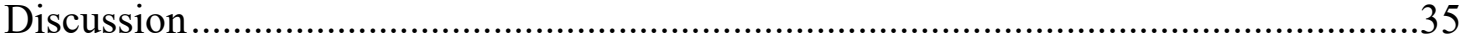

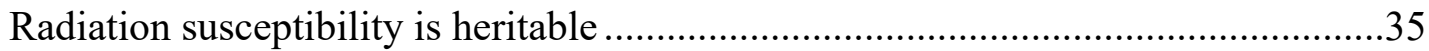

MST is linked to endothelial stem cells, inflammatory signal regulators and

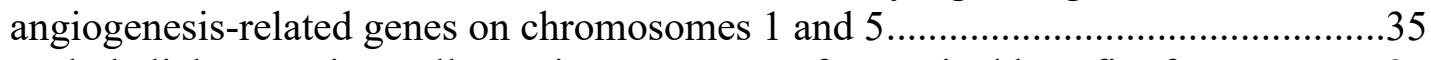

Endothelial progenitor cell recruitment may confer survival benefit of C57 ............37

Affymetrix mRNA correlations reveal genes associated with an angiogenicreparative response. 
Conclusions and perspectives

\section{CHAPTER 4. MODULATION OF RADIATION INJURY RESPONSE IN RETINAL ENDOTHELIAL CELLS BY QUINIC ACID DERIVATIVE KZ-41 ....39}

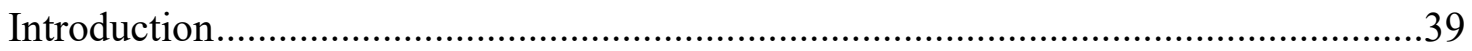

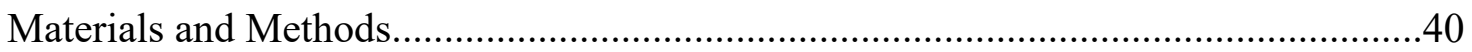

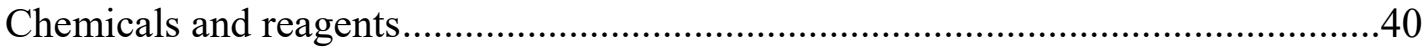

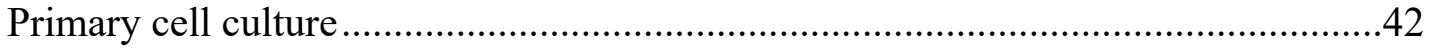

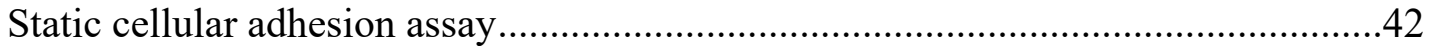

Parallel-plate flow chamber adhesion assay ....................................................42

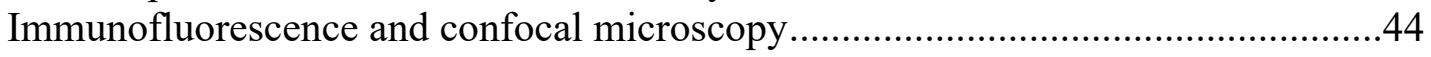

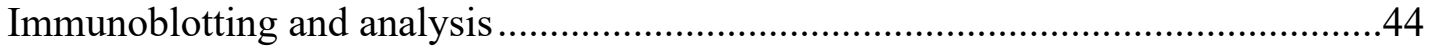

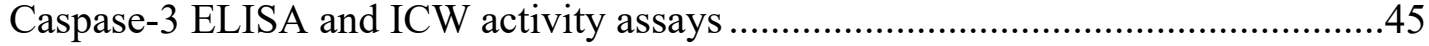

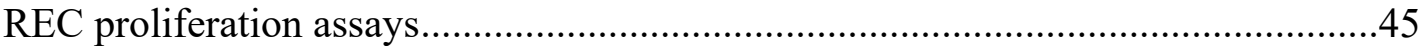

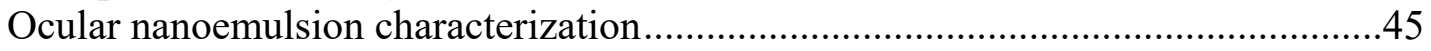

Murine oxygen-induced retinopathy model ...................................................46

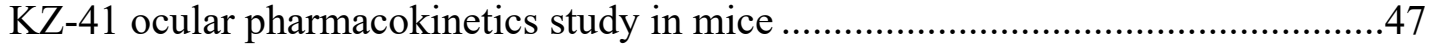

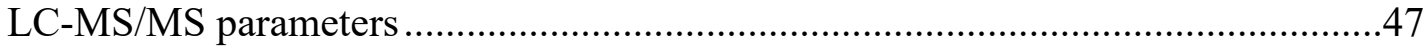

Preparation of calibration standards and quality control samples...........................48

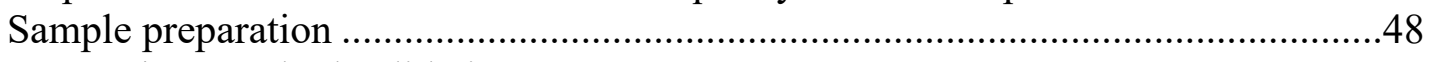

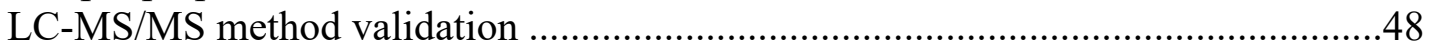

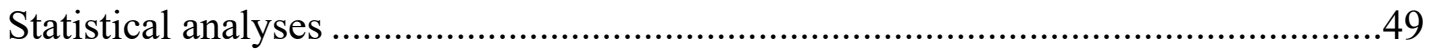

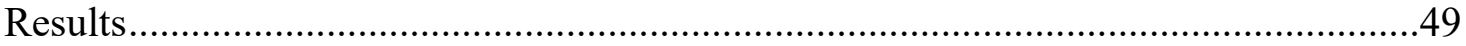

Radiation induces adhesion of U937 cells under static and dynamic flow

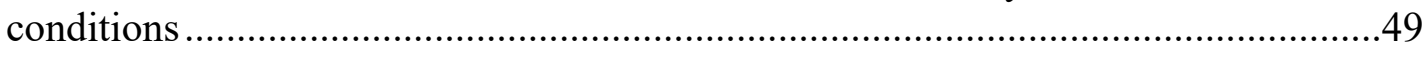

Radiation enhances ICAM-1 and sICAM-1 levels in RECs...................................51

KZ-41 inhibits radiation-induced ICAM-1 expression through a p38 MAPK-

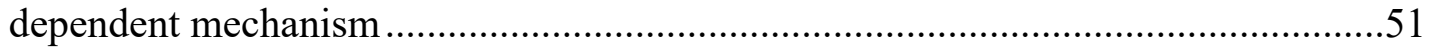

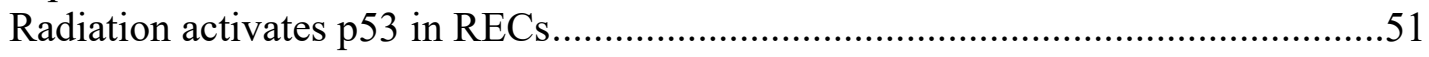

Radiation-induced REC death prevented with KZ-41 .....................................56

Radiation induces a paxillin-dependent proliferative phenotype............................56

KZ-41-loaded nanoemulsion delivered effective concentrations to mouse eyes.......56

KZ-41 reduces avascular area and neovascularization in the murine OIR model .....62

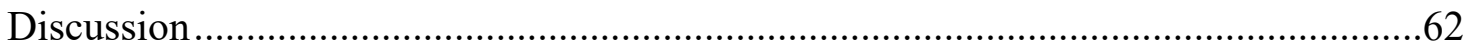

\section{CHAPTER 5. DEVELOPMENT OF PAXILLIN MODULATOR, JP-153 FOR} THE TREATMENT OF NEOVASCULAR EYE DISEASE ...................................71

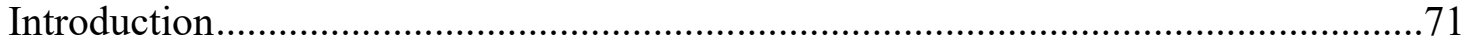

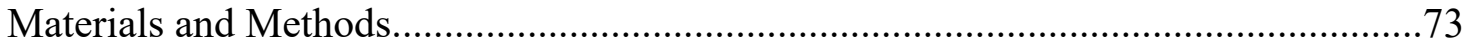

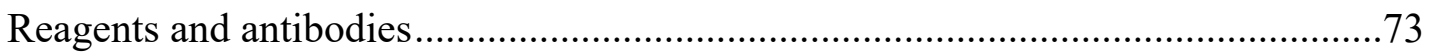

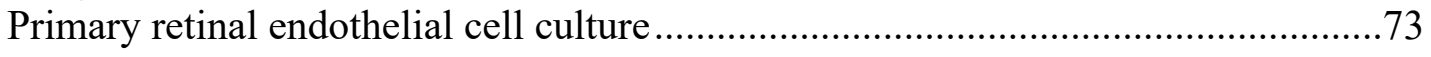

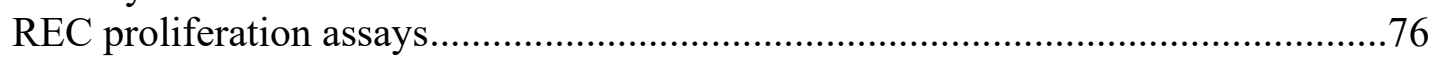

Annexin-V/FITC staining and flow cytometry analysis for apoptosis ....................76

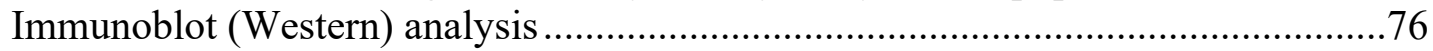

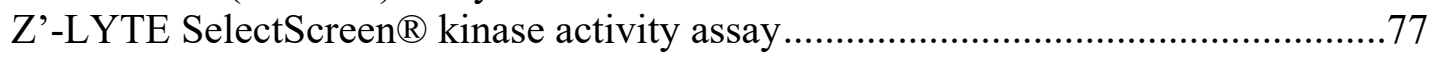

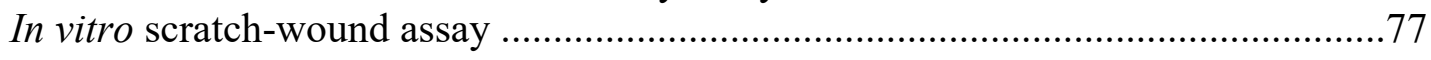


Transwell cellular migration assays........................................................................78

Retinal angiogenesis: murine oxygen-induced retinopathy (OIR) model ..................78

Retinal whole-mount imaging and analysis .........................................................79

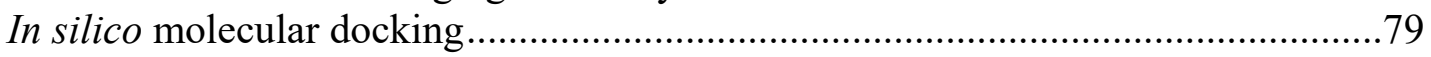

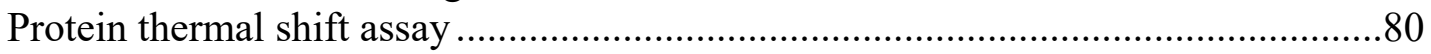

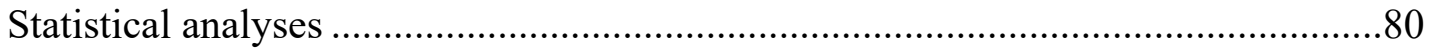

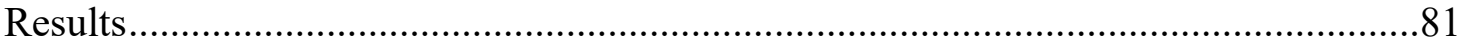

Src/FAK-paxillin signaling pathway in REC proliferation .......................................

Discovery of JP-153 as a potent inhibitor of VEGF-induced proliferation ................81

Effector signaling through an activated Src/FAK-paxillin complex is Akt-

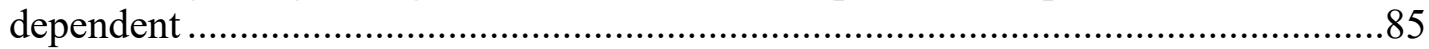

Paxillin modulation with JP-153 inhibits VEGF-induced migration of retinal

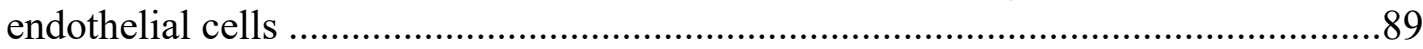

Molecular docking calculations suggest that JP-153 binds preferentially to FAT,

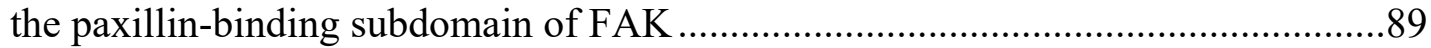

JP-153 in vitro target engagement of the FAT domain inconclusive .......................94 Disruption of Src/FAK/paxillin complex by JP-153 in vivo inhibits RNV in the murine oxygen-induced retinopathy model ..........................................................94

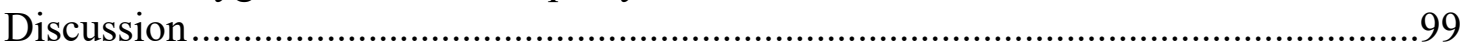

CHAPTER 6. PHARMACOKINETICS OF JP-153 ..................................................104

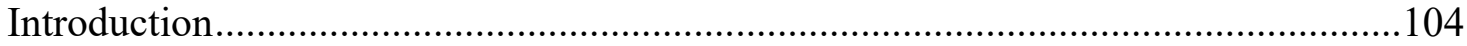

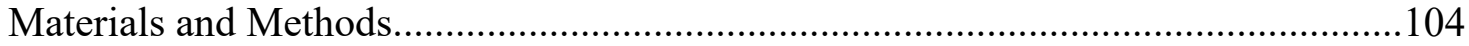

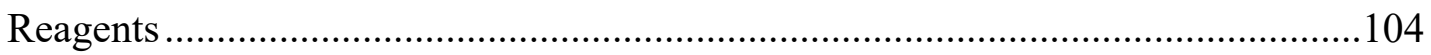

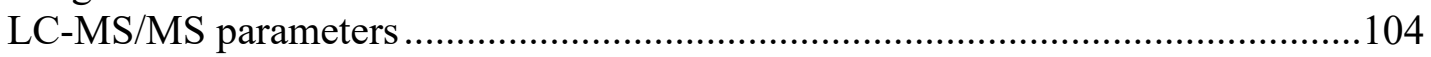

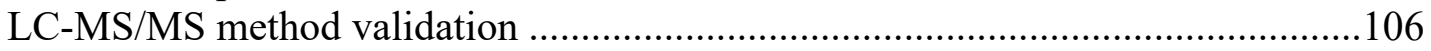

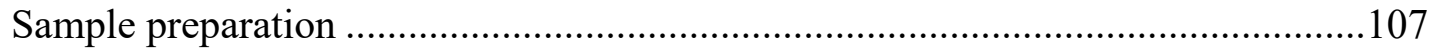

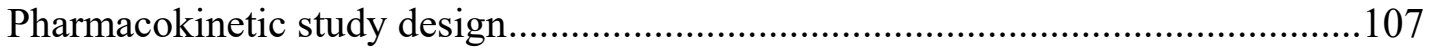

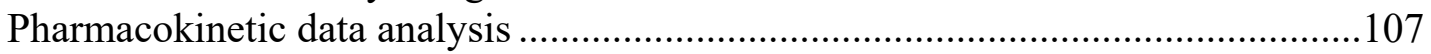

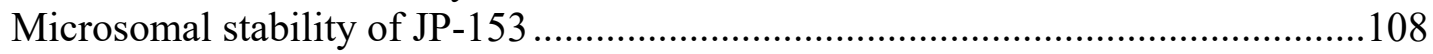

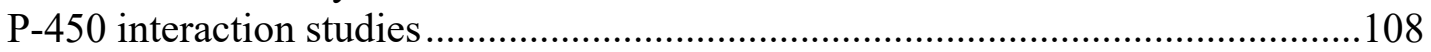

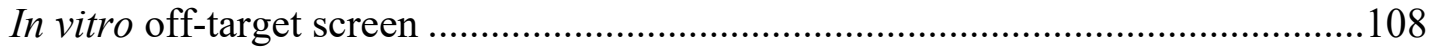

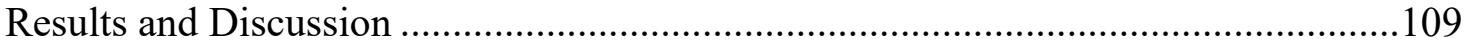

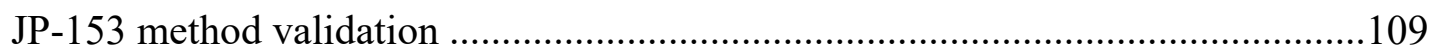

Intravenous and oral in vivo pharmacokinetics of JP-153 in Lewis rats...................109

In vitro ADME and off-target screening for JP-153 ……….................................109

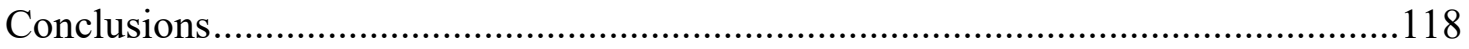

CHAPTER 7. SUMMARY ..............................................................................................119

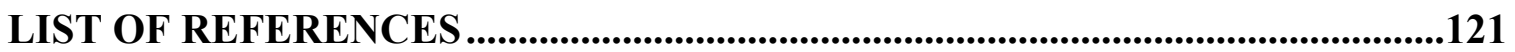

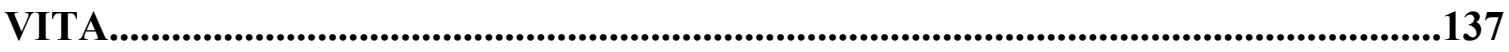




\section{LIST OF TABLES}

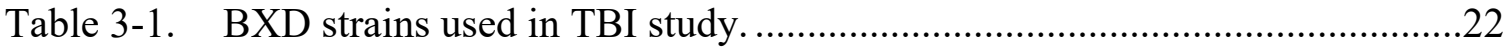

Table 3-2. BXD genes associated with suggestive QTL located on chromosome 1.....29

Table 3-3. BXD genes associated with suggestive QTL located on chromosome 5.....29

Table 3-4. Marker regression correlation analysis and additive effect. ........................30

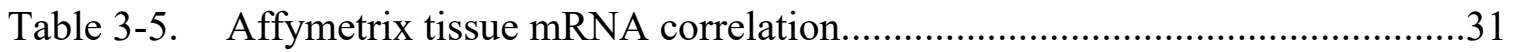

Table 4-1. Ophthalmic nanoemulsion characterization...........................................61

Table 4-2. Eye and plasma concentrations after ocular administration of KZ-41 ........65

Table 5-1. Primary antibodies used in the present chapter........................................74

Table 5-2. Z Z-LYTE SelectScreen ${ }^{\circledR}$ kinase inhibitor assay. .......................................8

Table 5-3. Melting temperatures (Tm) of increasing concentrations of FAT..............96

Table 5-4. Tm of the FAT peptide with increasing concentrations of JP-153 .............96

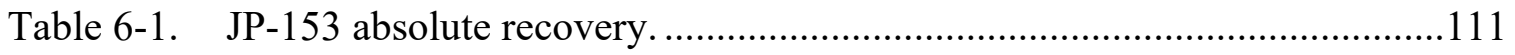

Table 6-2. Pharmacokinetic parameters of JP-153 after IV administration.................113

Table 6-3. JP-153 in vitro liver microsomal studies in human, mouse, and rat..........113

Table 6-4. CYP450 enzyme interaction studies: JP-153 versus control inhibitors......115

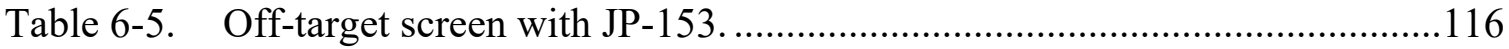




\section{LIST OF FIGURES}

Figure 1-1. Causative mechanisms of neovascularization in ischemic retinopathy.........4

Figure 1-2. Neovascularization in diabetic retinopathy and 'wet' AMD .......................5

Figure 1-3. Leukocyte-endothelial cell interactions. .............................................

Figure 1-4. Focal adhesion complex activity and downstream signaling.....................14

Figure 3-1. BXD recombinant inbred (RI) strain breeding schematic. .........................18

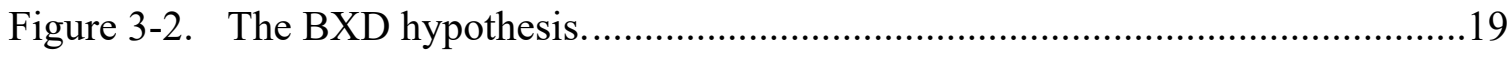

Figure 3-3. K-M survival plot of BXD parental strains C57BL/6J and DBA/2J..........24

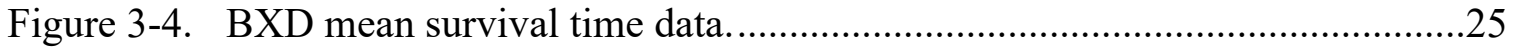

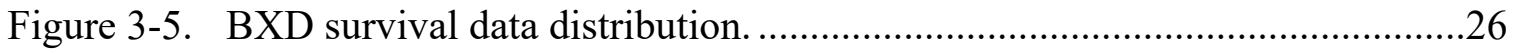

Figure 3-6. Preliminary QTL mapping of MST data among 40 BXD strains...............28

Figure 3-7. K-M survival curves of $\mathrm{C} 57 \mathrm{Bl} / 6 \mathrm{~J}$ mice exposed to $6.53 \mathrm{~Gy}$ radiation.........33

Figure 3-8. 6-B345TTQ reduced adhesion of U937 cells to irradiated endothelium......34

Figure 4-1. Chemical structures of KZ-41 and internal standard (IS) KZ-39. .............41

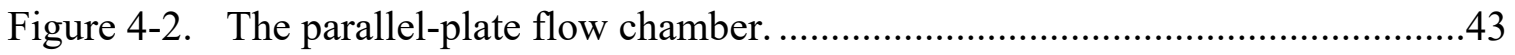

Figure 4-3. Radiation-induced U937-REC adhesion inhibited by KZ-41.....................50

Figure 4-4. Soluble ICAM-1 elevated by high-dose irradiation in RECs. .....................52

Figure 4-5. KZ-41 decreased levels of ICAM-1 following radiation in RECs. ..............53

Figure 4-6. KZ-41 inhibits IR-induced p38 phosphorylation in RECs. .........................54

Figure 4-7. KZ-41 reduced p53 protein levels in irradiated RECs.............................55

Figure 4-8. KZ-41 reduces IR-induced cleavage of apoptotic marker caspase-3...........57

Figure 4-9. VEGF secretion in REC media post-IR as measured by ELISA.................58

Figure 4-10. KZ-41 prevents IR-induced proliferative activity via paxillin. ..................59

Figure 4-11. Characterization of ocular nanoemulsion. ...........................................60

Figure 4-12. LC-MS/MS chromatograms of KZ-41.................................................63 
Figure 4-13. Intra-ocular and plasma concentrations of topical KZ-41.

Figure 4-14. KZ-41 reduces avascular area............................................................66

Figure 4-15. KZ-41 reduces neovascularization................................................67

Figure 4-16. Summary model of KZ-41 radioprotective mechanism-of-action...............70

Figure 5-1. VEGF-induced focal adhesion activation of endothelial cells....................72

Figure 5-2. 6-B345TTQ and JP-153 chemical structures and estimated Log P............75

Figure 5-3. VEGF-induced FA signaling in RECs............................................... 82

Figure 5-4. Src-dependent activation of FAK and paxillin in RECs............................83

Figure 5-5. Discovery of JP-153 as an inhibitor of VEGF-induced proliferation...........84

Figure 5-6. JP-153 inhibits VEGF-induced activation of paxillin Y118.......................86

Figure 5-7. JP-153 inhibits proliferation through Src/FAK/paxillin FA complex. ........87

Figure 5-8. JP-153 inhibited VEGF-induced REC migration. .................................90

Figure 5-9. JP-153 inhibited VEGF-induced REC invasion. ....................................91

Figure 5-10. JP-153 in silico docking between the FAT domain and LD motifs.............92

Figure 5-11. In silico interactions of JP-153 R and S enantiomers with FAT.................93

Figure 5-12. Protein thermal shift assay of the FAT domain.....................................95

Figure 5-13. JP-153 inhibited retinal angiogenesis in the murine OIR model................97

Figure 5-14. JP-153 does not affect vascularization in developing retinas. ....................98

Figure 5-15. Summary diagram depicting JP-153's proposed target of action. ..............103

Figure 6-1. Structures of JP-153 and internal standard (IS), BA-126 ...................... 105

Figure 6-2. Chromatograms of blank plasma and JP-153/IS spiked plasma................110

Figure 6-3. Average plasma concentrations of JP-153 in Lewis (LEW/Crl) rats..........112

Figure 6-4. JP-153 EC50 and IC50 analysis against $\mathrm{MT}_{1}$ and 5-HT2B receptors..........117 


\section{LIST OF ABBREVIATIONS}

AMD

ATCC

AUC

$\mathrm{AUC}_{\mathrm{IV}}$

AUCPO

$\mathrm{CL}$

CLF

$\mathrm{C}_{\max }$

DPBS

DME

DR

ELISA

EPC

$\mathrm{F}$

$\mathrm{IC}_{50}$

ICW

IR

IS

IV

LC-MS/MS

MAPK

MOE

MVGS

NE

NCA

NV

OD

OIR

PBS

PK

PO

QD

REC

RNV

RPE

RPMI

RR

SD

SEM

SNP

US FDA

USP

Vd
Age-related macular degeneration

American type culture collection

Area under the curve

Area under the curve, IV-dose

Area under the curve, PO-dose

Clearance

Oral clearance

Maximum plasma concentration

Dulbecco's phosphate-buffered saline

Diabetic macular edema

Diabetic retinopathy

Enzyme-linked immunosorbent assay

Endothelial progenitor cell

Oral bioavailability

Concentration required to produce half-maximal effect

In-cell western

Gamma $(\gamma)$-ionizing radiation

Internal standard

Intravenous

Liquid chromatography-mass spectrometry

Mitogen activated protein kinase

Molecular operating environment

Microvascular growth supplement

Nanoemulsion

Non compartmental analysis

Neovascularization

Optical density

Oxygen-induced retinopathy

Phosphate-buffered saline

Pharmacokinetic

Per oral

Once daily

Retinal endothelial cell

Retinal neovascularization

Retinal pigment epithelium

Roswell park memorial institute medium

Radiation retinopathy

Standard deviation

Standard error of mean

Single nucleotide polymorphisms

United states food and drug administration

United states pharmacopoeia

Volume of distribution 


\section{CHAPTER 1. RETINAL NEOVASCULARIZATION - CURRENT STATE OF TREATMENT AND STRATEGIES FOR NOVEL DRUG TARGETS}

Neovascularization (NV) is an essential process in embryonic, early post-natal development, and tissue repair, but can also contribute to pathological disease in adult humans including metabolic disorders, inflammation, cardiovascular abnormalities, and tumor development. In the most specific sense, NV is the growth of new blood vessels into avascular or ischemic tissue, which is distinct from angiogenesis where sprouting and growth from pre-existing vessels is requisite. However, both are characterized by the expanse of a vascular plexus that supplies hypoxic tissue with oxygenated blood under the tight regulation of signaling molecules, both temporally and spatially [1]. When signaling molecules are in abundance, as in pathologic disease, it is difficult to regain control over the angiogenic response, the major causative factor in ocular NV diseases, as the eye was not meant to be further vascularized during adulthood.

In the following sections, the distinctions and similarities between physiological and pathological neovascularization are discussed. Subsequently, we describe the clinical presentations of neovascular eye diseases associated with ischemic and degenerative retinopathies, along with mediators of their progression. We then rationalize how therapeutic interventions arose from pivotal studies and discuss their current limitations. We compare and contrast these therapies with special focus on alternatives entering the pre-clinical and clinical landscape, i.e., downstream signaling components of angiogenesis and anti-inflammatory targets.

\section{Physiological Neovascularization}

Physiological neovascularization represents an important component to both embryogenesis and post-natal development. Angiogenesis, similarly, is a rarity in adult humans, mainly being associated with the female reproductive system during menstrual cycles and pregnancy. In the adult retina, the vasculature is a mature network with very little endothelial cell turnover (frequencies measured in years). The stabilization and remodeling of the retinal vasculature occurs after early events of vasculogenesis where blood vessels are created de novo from endothelial progenitor cell (EPCs) precursors, or angioblasts. Through tightly controlled processes involving pro-angiogenic factors, the retina is supplied with an intricate framework of capillaries and pericytes (mural cells) which traverse the neural retina to support its function, integrity and overall health.

Endothelial cell growth and sprouting during development are sequential events that must occur in a step-wise manner to provide the necessary stability needed to ensure proper blood flow in tissues. Initially, pericytes which are cells that form a barrier between the endothelium and surrounding tissues, are shed from the branched endothelium to allow for vessel sprouting. New vessel projections first require a proteolytic degradation of the existing basement membrane for which a new matrix foundation must be laid down. Stromal cell-derived matrix then allows for the 
proliferation of endothelial cells. Soluble factors in and around the area activate and regulate endothelial cell proliferation, lead migration and help form a new monolayer into tube-like structures. Pericytes are once again recruited to stabilize these new formations and allow for the restoration of blood flow to anoxic tissues. Pericyte-mediated stabilization has been described as the end of the so-called "plasticity window" where blood flow is finally restored and the vasculature is fine-tuned to meet the oxygen demands [2].

The de novo establishment of endothelial cells from EPCs arise to generate the vascular plexus in a process called vasculogenesis, which occurs early in embryonic development. In adulthood, the bone marrow contains stores of precursor stem cells and aides in the tissue repair and regeneration following an injury. Mobilization of bone marrow-derived EPCs to uninjured organs and stromal tissues provides a sort of "maintenance reservoir" to help aide in reparative vascular homeostasis [3]. In fact, it is known that EPCs comprise up to $25 \%$ of endothelial cells in new vessels in animal models. Thus, EPCs represent an important component to vasculogenesis both during development and in adult hood as a way to maintain organ health and facilitate tissue repair [4].

Vasculogenesis, and angiogenesis are processes that are tightly regulated by numerous pro- and anti-angiogenic factors, and is effectively halted under nonpathological circumstances once the oxygen demands of tissues are met. It is believed that there are over 50 interdependent growth factors, cytokines, and enzymes that contribute to the regulation of vessel growth [5]. Many of these factors are considered to be a part of the families of the vascular endothelial growth factors, VEGF, angiopoietins, and ephrins [6,7]. VEGF is the most notable of all angiogenesis inducers, and its absence in knockout mice leads to early embryonic death within 10 days [5]. However, proper balance of both inducers and inhibitors of angiogenesis is a necessary component in a well-defined and organized vasculature.

\section{Pathological Neovascularization}

Pathological neovascularization and angiogenesis are processes by which the balance of pro- and anti-angiogenic signals is shifted and the necessary regulatory mechanisms are ignored. NV diseases circumvent these feedback checkpoints, either through disruption in the strict regulation of angiogenic factors, or through injury that can disrupt the normal homeostasis of endothelial cell and pericyte-dependent regulation. These events are consistently linked to disease progression in diabetic patients occurring due to loss of endothelial cells and pericytes stemming directly from injuries associated with high levels of blood glucose. Acute injury stemming from radiation exposure also exhibits similar pathological tendencies. Moreover, failures of endothelial protection and pericyte support in diabetics can increase the likelihood of radiation retinopathy in patients undergoing radiotherapy. It seems likely then that the role of the strict regulation and health of the microvasculature is inherently linked to survival of endothelial cells and pericytes. Injury that causes loss of endothelial cells and pericytes leads to an ischemic 
retina which triggers immune cell adhesion, EPC homing and repair of hypoxic tissues. In diabetic retinopathy, it is widely accepted that altered homeostasis and dysfunction of EPCs contribute to the pathogenesis [8-10]. There is still some debate as to how EPCs contribute to the pathogenesis of ischemic retinopathy, as the relationship between numbers/function of EPCs with the progression of DR is unclear [8]. However, it is believed that injuries which activate repair responses in the retina incite further damage, leading to an endless cycle of inflammation, cellular death, and ischemia (Figure 1-1).

In later stages of ischemic retinopathy and retinal degenerative diseases, growthfactor induced angiogenesis ultimately contributes to the loss of visual function. In diabetic retinopathy and radiation retinopathy, inflammation-mediated capillary occlusion generates areas of ischemic tissue. In age-related macular degeneration, AMD, a balance of growth factors in and around the retinal pigment epithelium (RPE) is disrupted by breaches in the Bruch's membrane leading to deregulated vessel growth (Figure 1-2) [11]. Pathological retinal angiogenesis occurs at such a rapid rate in these diseases, as compared to normal vasculogenesis, that vessels are more prone to leakage and hemorrhages which further promotes the ischemic/hypoxic injury and continues the chronic cycle of damage and dysfunctional repair.

While blindness from NV eye disease is detrimental to quality of life, tumor angiogenesis represents a life-or-death scenario when tumors hijack the body's own angiogenic response, using similar mechanisms of unregulated vascular development seen in retinal diseases $[12,13]$. Tumors are inherently hypoxic once they reach a certain threshold for nutrient demands. Promoting vascularization in cancerous tissue, in effect provides tumors with undefined growth potential, a prognosis of the grimmest kind. While these chapters do not discuss tumor angiogenesis in much detail, it still remains one of the most important aspects of cancer treatment.

\section{Ischemic Retinopathy}

\section{Overview}

Ischemic retinopathies and related degenerative eye diseases, such as age-related macular degeneration (AMD) are the most common causes of adult-age blindness. Stemming from an inflammatory insult(s), ischemia affects the retinal tissues by inciting a cascade of events that trigger growth of new blood vessels in the pre-retinal space and the choroid. It is ischemia that is thought to perpetuate a life-long condition of cellular death $\rightarrow$ inflammation and leukostasis $\rightarrow$ capillary closure and dropout $\rightarrow$ hypoxia and pathological neovascularization. These events are hallmark features seen in diabetic patients and those undergoing radiotherapy for various ocular and head/neck cancers. 


\section{Radiation AMD Diabetes}

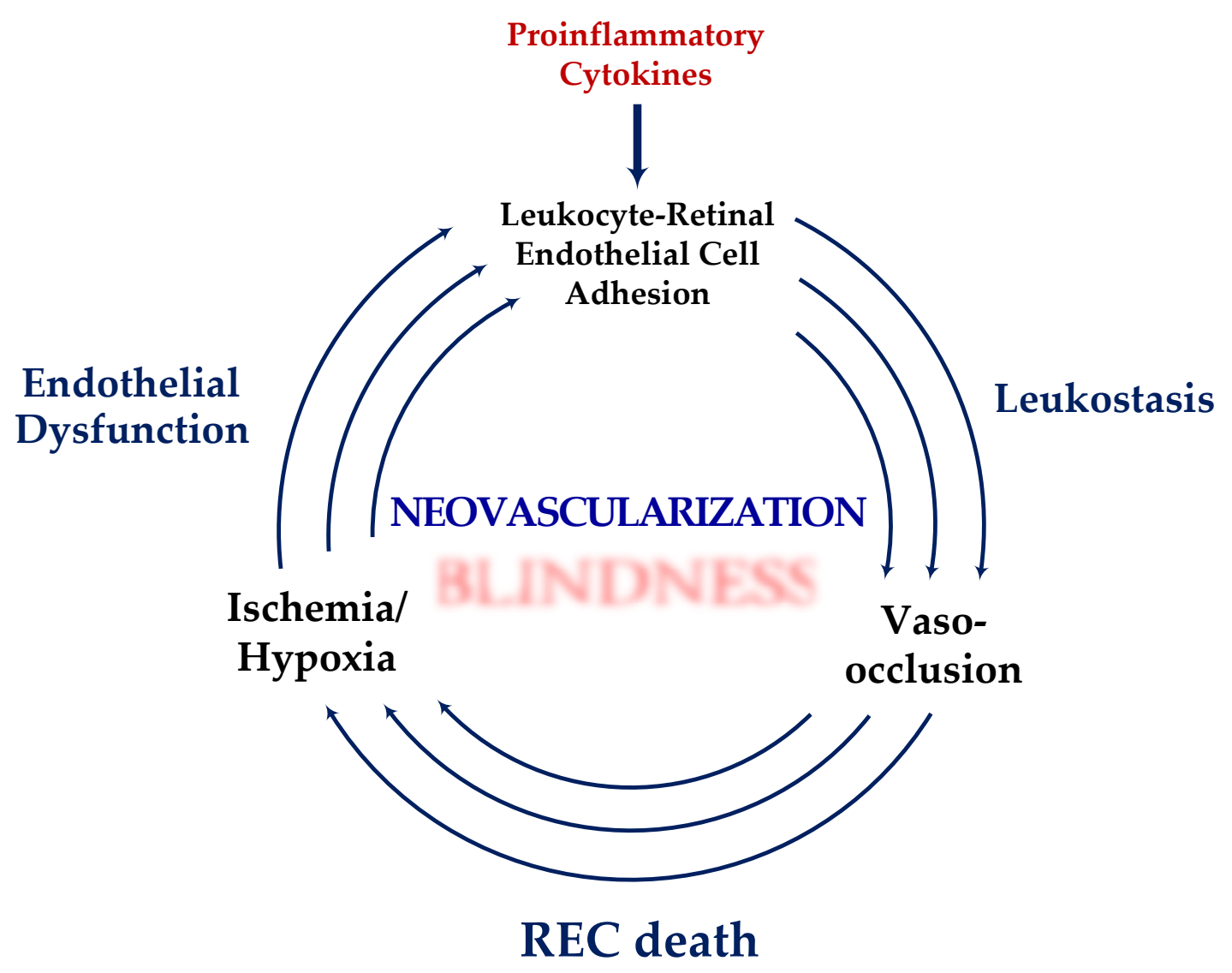

Figure 1-1. Causative mechanisms of neovascularization in ischemic retinopathy.

Damage inflicted by either diabetes, age or radiation exposure to the vascularized retina triggers an exuberant pro-inflammatory response resulting in leukostasis, vessel occlusion, retinal endothelial cell (REC) death, and subsequent hypoxia. A hypoxic retina then triggers neovascularization to try and bring oxygen supplies back to the starved, dying tissue. Neovascularization occurs hastily and without the proper organization that leads to vascular vulnerabilities, including hemorrhages and neuroretinal damage leaving many adult patients with severe vision loss. 


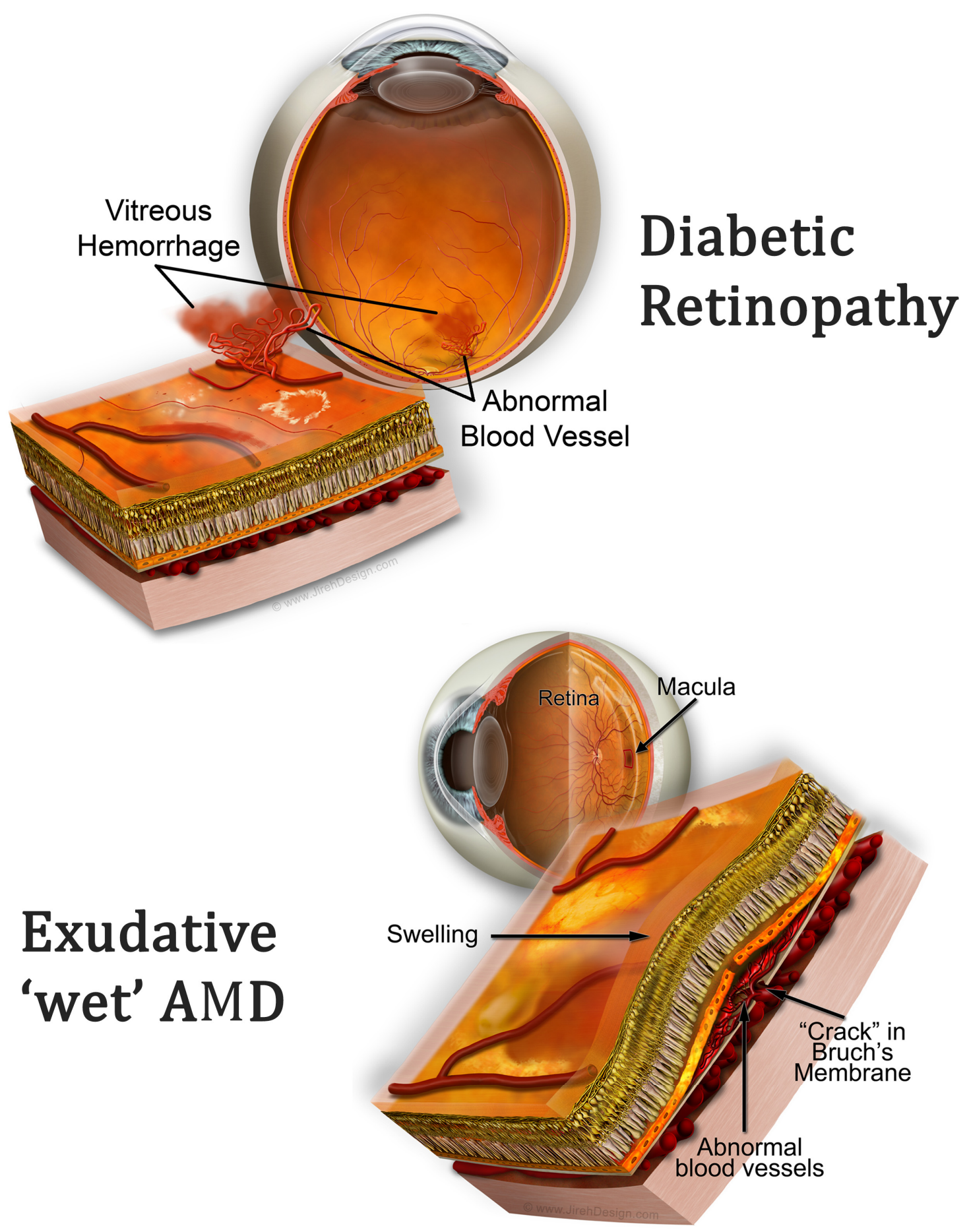

Figure 1-2. Neovascularization in diabetic retinopathy and 'wet' AMD.

Figure modified with permission from Erickson, M., Medical Eye Illustrations. 2016: jirehdesign.com. 


\section{Leukocyte-retinal endothelial cell interactions}

Initial inflammatory response to vascular injury activates adhesion molecules on surfaces of endothelial cells which allows for immune cells to localize to damaged tissues. Severe microvascular injury has been touted as the primary mechanism in the pathogenesis of ischemic tissue damage in diabetic retinopathy (DR) and radiation retinopathy (RR) [14]. Specifically, studies have emphasized mechanisms of retinal injury as comprising leukocyte entrapment, accumulation within microvessels, and capillary closure [15]. Compared to non-diabetic retinas, leukocyte adherence is substantially increased in venules, arterioles, and capillaries [16]. Under high shear blood flow, tethering and rolling are initiated by selectins (e.g., L-, P- and E-selectin) which are rapidly presented to the cell surface in response to inflammatory stimuli [17]. Initial activation of an inflamed endothelium relies upon selectins to initiate the "rolling" phenomena of circulating immune cells. The act of tethering allows the rolling cells to slow down and intercept immunoglobin superfamily of cellular adhesion molecules, ICAM, VCAM, PECAM, etc. to promote firm adhesive-like interactions causing immune cells to become stationary (Figure 1-3) [18]. ICAM-1 is one of the most recognizable initiators of leukocyte-endothelial cell adhesion [19] and has been highly correlated with ocular inflammatory disorders [20-24]. ICAM-1 together with focal adhesion and celljunction proteins facilitates the adhesion and transmigration of circulating leukocytes into the site of tissue damage.

\section{Retinal endothelial cell death and dysfunction}

Leukocyte adhesion can trigger endothelial cell dysfunction and even cell death in diabetic patients. It is also the primary injury associated with acute radiation-induced vascular damage and permeability [25]. However, pathological mechanisms of leukocytemediated endothelial cell death in ischemic retinopathy are not completely understood in humans, as they can occur without clinical detection for years before vision loss occurs. It is thought though, that the frequency and extent of leukocyte adhesion triggers irreparable breakdown of the blood-retinal barrier, loss of supporting pericytes, and deterioration of gap-junctions of the endothelium through leukocyte-dependent secretion of permeability factor, VEGF. Ultimately, these events lead to acellular "ghost" capillaries, or basement membrane tubes devoid of pericytes and viable endothelial cells. In rodents, the absence of ICAM-1 drastically reduced leukocyte adherence to the endothelium in long-term diabetic studies and almost completely suppressed the BRB breakdown associated with leukocyte-endothelial cell interactions [26].

Pivotal studies in diabetic animal models, showed that interacting leukocytes can trigger apoptosis of endothelial cells directly though the Fas/FasL pathway. Fas receptors, also known as Apo-1 or CD95, are a part of tumor necrosis factor (TNF) and nerve growth factor (NGF) receptor families [27, 28]. Activation of this pathway in microvascular cells cause widespread apoptosis that is believed to be the mechanism behind the BRB breakdown in DR [29]. Additionally, diabetes-induced retinal ganglion cell and astrocyte death was prevented with antibodies reactive against Fas/FasL pathway, further implicating the Fas/FasL pathway in vision loss in ischemic disease. 


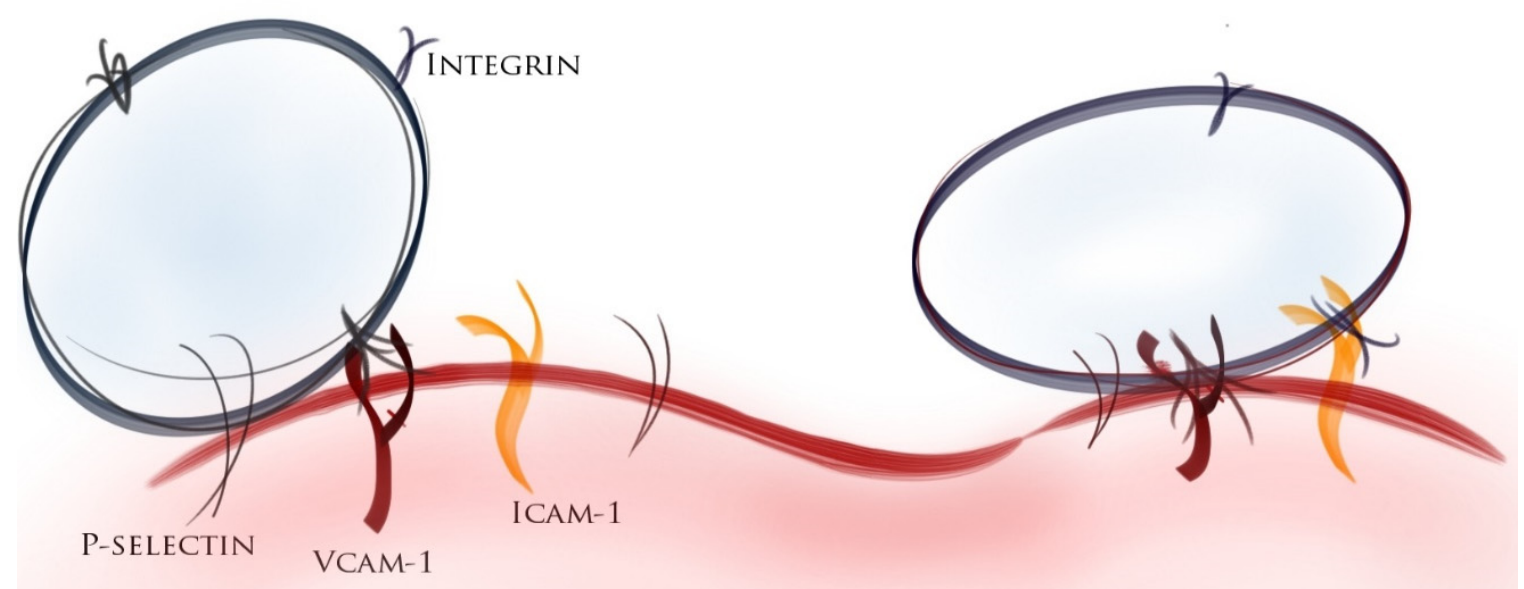

\section{Figure 1-3. Leukocyte-endothelial cell interactions.}

Leukocyte rolling is initiated by surface selectins, which slows down the circulating cell to allow for tethering and subsequent firm adhesion through leukocyte integrins and immunoglobin surface proteins, ICAM-1 and VCAM-1. A firmly adhered immune cell will transmigrate towards the damaged or inflamed tissue, the so-called extravasation process. These phenomena are touted as the primary pathological event that occurs during ischemic retinopathies. 


\section{Hypoxia-triggered $\mathrm{NV}$}

There is an active debate in the literature as to whether or not ischemic DR begins with abnormalities in the neuronal and glial cells of the retina or in the retinal blood vessels. Regardless of the initiating event(s), it is clear that microaneurysms in the retina represent the first clinically observable manifestation of disease [30]. Microaneurysms are associated with REC and pericyte loss, which leads to capillary dropout and development of retinal ischemia. Infiltration of leukocytes to injured tissues contribute to and even promote NV in ischemic disease [31].

Damage to the vascular retina results in vessel blockage, decreased oxygen supply, and retinal endothelial cell (REC) death [14-16]. Incidences of occlusion and diminished oxygen initiate aberrant vessel growth, or retinal neovascularization due to a barrage of secretory inflammatory cytokines. Clinical presentation of ischemic retinopathies comprises hemorrhages, microaneurysms, and other consequences of a leaky microvasculature. Hallmark features of ischemic retinopathy are quite similar to other chronic degenerative retinal diseases (e.g., diabetic macular edema, age-related macular degeneration, AMD). In fact, recent reports cite that the risk of developing RR are significantly higher in patients with comorbid diabetes, suggesting an additive component in the pathology of ischemic retinopathy [32].

\section{Radiation retinopathy}

Radiation retinopathy (RR) is a chronic degenerative disease that leads to significant visual impairment [33, 34]. RR results from exposure of the eye to various radiotherapy interventions (e.g., external beam, plaque brachytherapy, and gamma knife) [35-39]. RR is also prevalent in patients undergoing treatment for nasopharyngeal or brain cancers due to incidental exposure during treatment [40]. The progression of RR was recently reported as $5.8 \%$ at five years and $7 \%$ at ten and fifteen years, and in 3,841 eyes treated with plaque radiotherapy for uveal melanoma [41]. Consequently, RR incidence is currently estimated between $3-20 \%[42,43]$.

Radiation exposure to delicate tissue, such as the retina is of major concern in patients receiving radiotherapy. Consequently, exposures of ionizing radiation to ocular tissue is associated with high risks of irreversible damage to vision and can severely impact a patient's quality of life. Nevertheless, radiotherapy is still currently the primary treatment options for intraocular and intracranial tumors due to lower recurrence rates and limited acute side-effects. Radiotherapy also represents an important alternative in the treatment of these tumors, since it provides equivalent or enhanced survival compared to enucleation (eye removal) [44, 45]. There are no treatment options, before or after, for ameliorating the initial damage associated with radiation injury. It is therefore imperative to find solutions for patients; one, that can protect their eyes from incidental damage, and two, help resolve the inflammation upon such an exposure. 


\section{Diabetic retinopathy}

Diabetic retinopathy (DR), the most frequently occurring microvascular complication of diabetes, is a leading cause of vision loss. The clinical severity of DR is characterized by retinal vascular occlusion and has been attributed, in part, to impaired collateral vessel development and leukostasis. Retinal microaneurysms, an early disease manifestation, are associated with REC death, capillary dropout, and macular edema [30]. The resultant ischemia triggers hypoxia-induced factor-1 (HIF-1) driven VEGF expression, which is a biomarker of retinal neovascularization (RNV) [46]. Acellular capillary formation in response to hypoxia exacerbates vascular leakage thus propagating a cycle of capillary death, ischemia and pathological RNV.

Prolonged high glucose exposure inactivates pro-survival signaling leading to reduced endothelial cell viability [47]. Inflammation and subsequent leukocyte invasion contribute to the breakdown of the blood-retinal barrier, the thickening of the basement membrane, and ultimately contributes to the dysfunction and death of RECs, pericytes and vascular smooth muscle cells in progressive DR. Mechanisms of glucose injury comprise several interrelated pathways involving oxidative stress, PKC activation, stressactivated p38 MAPK, nuclear factor-kB (NFkB), and the NH2-terminal Jun kinases, in addition to the enhanced secretion of pro-inflammatory cytokines TNF $\alpha$ and VEGF.

Diabetic retinopathy is strongly associated with the pro-survival effects of Akt on REC death in early stages of high-glucose mediated retinal injury. Additionally, it makes sense that this gene is of potential importance in IR-induced injury, an acute insult akin to that of chronic hyperglycemia. In fact, through growth factors, Akt regulates endothelial cell survival which plays an important role in tissue repair, but can also contribute to pathological neovascular disease [48]. However, during angiogenesis, Akt is a vital component to focal adhesion signaling which contributes to the later stages of pathological neovascularization in DR and other ischemic/neovascular eye diseases [49].

REC dysfunction and injury are also attributed to various other mediators, one being the insulin-like growth factor 1 (IGF-1)/IGFBP (IGF binding protein) autocrine system [50]. IGF binding proteins consists of seven different binding protein isoforms that are thought to control the release and distribution of IGF-1 into interstitial fluids. IGFBP-3 binds directly to IGF-1 to facilitate its delivery and concentration to sites of ischemic injury. However, there is still some debate as to whether IGF-1 itself contributes to the pathology of DR since it has been shown to be co-expressed with that of IGFBP levels in diabetic retinas. In diabetic rats, subcutaneous IGF-1 administration decreases retinal apoptosis as evidenced by a reduction in TUNEL-positive cells in the photoreceptor, inner nuclear, and ganglion cell layers [51]. IGF-1 bound by IGFBPs is unable to activate its receptor system, essentially being trapped in complex. Therefore, the assertion that IGF-1 contributes to the disease is somewhat misleading, since "free" IGF-1 is potentially unavailable for injured cells in a ratio that would benefit the retina. A better understanding of the mechanisms contributing to glucose-induced REC death may provide more insight into how diabetic disease progresses which may shed light on new targets for DR. 


\section{Pharmacologic Targets of Neovascular Disease}

Pathological angiogenesis is a primary driver of many diseases, ranging from ocular disorders to cancer. Like an ischemic retina in DR and RR, tumors are also hypoxic and starved for supplies of nutrients and oxygen. In order to sustain their high metabolic demands, they hijack the normal processes of angiogenesis to feed their expanse. Historically, many strategies to eliminate cancer have been to target cancer cells specifically, but due to numerous failed clinical trials, it became apparent that such a strategy was giving rise to unique resistance mechanisms, allowing tumors to evade targeted therapy [52]. Strategies soon evolved to incorporate a new, indirect strategy of antiangiogenic therapy which focused on nutrient supply chains, or the vasculature. With this approach, oncologists no longer needed to limit their attention to the individual cancer cell but could instead target the essentials of tumor development through angiogenesis.

The notion that tumors could be treated by targeting blood vessels started in the 1970's by angiogenesis pioneer and clinician scientist, Judah Folkman. He posited that solid tumors could nourish themselves by hijacking the body's blood supplies. This notion was met with great skepticism and at times, visceral objections from his peers. Nevertheless, his hypothesis soon paved the way for the advances in antiangiogenic research and has led to numerous patents, FDA approved therapeutics, and a new frontier in treating neovascular disease in humans.

It is virtually impossible to discuss angiogenesis without referencing the eye. It was Folkman himself who asserted, "In order to prove that a tumor could make a protein that could diffuse from the tumor and stimulate new capillaries, the only way we could show that was in the eye" [53]. Today, retinal development and neovascular disease models are essential to study how tumors develop blood vessels and these studies have led to a more comprehensive understanding of the mechanisms driving angiogenesis.

\section{Vascular endothelial growth factor, VEGF}

VEGF is crucial to normal vascular development, and has been the subject of extensive studies for its contribution to NV disease. Largely studied in this vein, VEGF is the target of many therapeutics aimed at sequestering its activity during angiogenesis, especially in ocular disease and cancers; though, it has also been considered for off-label use to facilitate surgical operations as a way to limit bleeding and reduce the length of surgery time [54]. Recent developments in VEGF research have begun to reconcile the seemingly paradoxical physiological and pathological relevance of VEGF as at least 12 different VEGF splice-isoforms comprising the major families, VEGF121, VEGF164, and VEGF188 have been discovered, which possess both pro- and anti-angiogenic activity [55].

VEGF isoforms have led to more selective therapeutics targeting VEGF-A 164, which is considered the primary driver of pathological angiogenesis, while VEGF 121, 
185 are believed to be more relevant in physiological angiogenesis. Each isoform plays a distinct role in vascular development where their ratios are shifted depending on the nature of disease and development of spatial proximity in tissues. These distinctions opened up a new series of approaches investigating how and why these isoforms differ in their activities, giving researchers and clinicians a much clearer picture for strategies to modulate angiogenesis in health and disease [56].

VEGF is known to induce the expression of inflammatory markers and its presence in other hyper-proliferative diseases is strongly correlated with enhanced tumor invasion and metastasis. However, targeting VEGF through antibodies or other protein therapeutics has been met with challenges in recent years. FDA approved anti-VEGF protein therapeutics are commonly used to treat complications of neovascular disease, including diabetic macular edema and wet AMD. Unfortunately, NV disease is not curable with these agents and therefore requires long-term therapy. Prospective studies indicate as many as $50 \%$ of patients treated with anti-VEGF agents fail to respond to initial treatments, and those that do, eventually lose efficacy over the years. In neovascular eye disease, for example, studies show a decline in visual improvement with long-term anti-VEGF treatments. This is thought to be due to failures in addressing the influence and expression of other growth factors and cytokines that activate similar signaling cascades $[52,57]$. Moreover, deterioration in vision can also be linked to the removal of the necessary support mechanisms for the neural retina. Although VEGF receptors have been identified in neuronal cells indicating the potential importance in maintaining proper visual function, the neuroprotective role of VEGF is not fully understood [58, 59]. The RPE-derived VEGF support system was found to be crucial for the survival and protection of photoreceptors and müller cells, giving more weight to the notion that long-term anti-VEGF treatments may have a negative impact on vision [60, $61]$.

\section{Endothelial progenitor cells}

In the developing embryo and in adult tissues responding to ischemic injury, mobilization of bone marrow (BM) derived endothelial progenitor cells facilitates the birth of new vascular networks (vasculogenesis/neovascularization) which replenish anoxic tissues with nutrient supplies. After the basic foundation of vascular labyrinths are created, angiogenesis takes over generating a cooperative landscape of pericytes, endothelial cells, and smooth muscle cells that innervate tissue.

During embryogenesis, there are thought to exist two subsets of vascular progenitor cells that vary in their angiogenic capacity. This notion led to the discovery of two vascular progenitor cell lineages, one comprising only an angioblastic capacity and the other which can actually differentiate into endothelial cells or hematopoietic cells [62]. A common lineage among endothelial cells of various tissues is supported by the shared expression of markers, including VEGFR-2, CD34, AC133 (prominin 1), PECAM-1, c-Kit and Sca-1. An intriguing finding was discovered in that when VEGF signaling was silenced in these progenitor cells, differentiation still occurred [5]. In fact, 
when vessels are mature, the expression of EPC marker AC133 is lost, making this marker ideal in distinguishing between EPCs and matured endothelial cells.

EPC mobilization from BM, as well as the recruitment to ischemic injury in adult vasculogenesis $[63,64]$ is initiated by numerous factors, including VEGF, FGF-2, PIGF and recently discovered platelet-derived growth factor, PDGF, in addition to the angiopoietin family (Ang-1 and Ang-2) [65]. In diabetic retinopathy, for example, studies have linked the pathogenesis of NV disease to the dysfunctional regulation of EPCs [8]. The ability of EPCs to mobilize from BM and localize to ischemic tissue regulates the presence of endothelial cell integrins. Notably, integrin $\alpha 4$ plays a highly specific role in $\mathrm{BM}$ retention and mobilization, as well as proper homing and adhesion to angiogenic sites through interactions with its receptor, VCAM-1 [66, 67]. Functional blockade of integrins is thought to enhance neovascularization in ischemic animal models, as enhanced mobilization of EPCs is promoted [68]. However, these studies showed that inhibition of integrins did not halt angiogenesis induced by mobilized EPCs. Therefore, targeting subcellular mechanisms related to integrins and growth factor signaling may be a solution. Focal adhesion proteins regulate the cooperative signals induced by integrins or growth factor receptors and are currently being investigated for their antiangiogenic properties. It was recently discovered that the expression levels of FA protein, paxillin is upregulated in AC133 expressing cells [69], though no studies targeting this protein exists to date. We explore this hypothesis in later chapters of this dissertation.

As neovascularization in human disease is commonly treated with anti-VEGF therapeutics, failures that arise in long-term treatments could be explained by the fact that EPCs are still able to inhabit, differentiate and form aberrant blood vessels in the human retina. Mouse models of pathological angiogenesis in different backgrounds have considered the incorporation of EPCs in growing vessels as being extremely high, or negligibly low $[70,71]$. Thus, variable genetics among mouse strains may serve as a way to decipher the primary mechanism of EPCs in contributing the pathological disease. In recent years, the role of EPCs in developing tumor microvasculature has gained traction, suggesting these may be useful biomarkers for monitoring the antiangiogenic efficacy of cancer therapeutics [72]. Targeting EPC directly may serve as a way to both combat antiVEGF resistance and effectively halt progression of $\mathrm{NV}$ disease.

\section{Focal adhesion complex}

Targeting kinase activity of FA proteins is an effective way to reduce angiogenesis as well as cancer growth in numerous pre-clinical and clinical studies, but there appears to be accumulating evidence of resistance as the cell circumvents druginduced deficits [57]. Thus, there is a critical need to identify drug targets that serve as "interface points" shared by multiple FA signaling proteins in angiogenesis.

Crosstalk between growth factor receptors and cell surface integrins finely regulate FA assembly and disassembly at the leading and trailing edge of the cell, respectively. Growth factors transmit signals through focal adhesion complexes or 
mechanotransduction "signalsomes" consisting of adaptor proteins and kinases, e.g., Srcfamily kinases, paxillin, and focal adhesion kinase (FAK) [73-75]. Paxillin, together with Src and FAK, recruit other proteins to the cell's leading edge where actin filaments coalesce around integrins, or "anchors" to provide contractile forces needed to pull the cell forward. Cells without paxillin show dysfunctional cell spreading and stunted migration and cells without FAK also show similar deficits [76-78].

Autophosphorylation of FAK at tyrosine 397 (Y397) facilitates the binding of the SH2 domain of Src, a non-receptor tyrosine kinase [76, 79]. The Src/FAK complex in turn further phosphorylates FAK in order to target downstream binding partners for FA assembly, including paxillin [77]. FAK brings Src in proximity to paxillin leading to Srcmediated paxillin Y118 phosphorylation, which targets the complex for recruitment to nascent FAs [80, 81]. These events occur within a tightly regulated nascent FA and disruption of any aspect of binding can greatly affect the complex's behavior and signaling mechanisms during angiogenesis (Figure 1-4) [82].

Paxillin, a multi-domain adaptor protein, can bind to multiple proteins including integrins and the four-helix bundle-containing FA proteins, FAK, proline-rich tyrosine kinase-2 (PYK2/FAK2), G-protein couple receptor-kinase interacting protein-1 (GIT-1), vinculin, and actopaxin [83]. Numerous studies characterizing these protein-protein interactions at the structural level have identified highly conserved sequences, or so called paxillin-binding subdomains (PBS) that co-crystallize with paxillin's leucine-rich domains (LD) $[84,85]$. The C-terminal end of FAK, or so-called focal adhesion targeting (FAT) domain, directly binds paxillin LD2/LD4 [86]. It is suggested that LD-PBS interactions promote localization of the complex to integrins at the cell surface during angiogenesis. It was recently shown that cells deficient in FAK protein were able to counter this loss by inducing the expression of PYK2 - also containing a FAT-like domain at its $\mathrm{C}$-terminal end, inferring a potential drug-resistance/rescue mechanism in anti-angiogenic therapies [87]. Therefore, targeting FAT-associated PBS with small molecules may be the key to overcoming anti-angiogenic resistance for the treatment of $\mathrm{NV}$ disease. In Chapter 5, we explore paxillin as a viable anti-angiogenesis target and show crucial evidence for its role in processing angiogenic signaling through VEGF. 


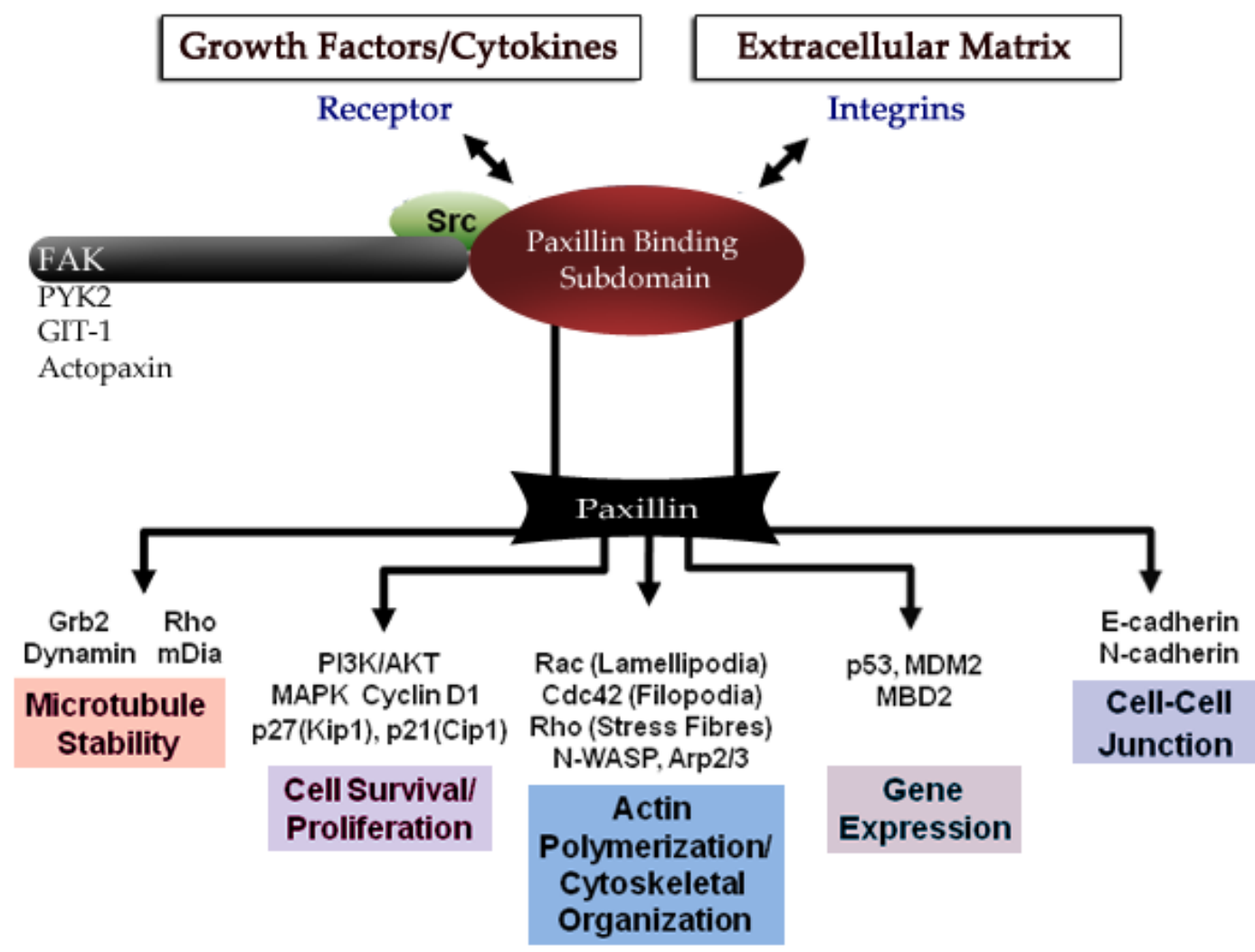

Figure 1-4. Focal adhesion complex activity and downstream signaling.

Focal adhesion kinase, FAK; Proline-rich tyrosine kinase-2, PYK2; GPCR-kinase interacting protein-1, GIT-1; Integrin-linked kinase, ILK; Paxillin-kinase linker, PKL 


\section{CHAPTER 2. CENTRAL HYPOTHESIS}

The overall objective of these studies was to identify pathophysiological targets in ischemic retinopathy and subsequent pathological retinal angiogenesis. We approached three specific aims with the central hypothesis that targeting inflammatory and angiogenic pathways related to endothelial cell focal adhesion (FA) protein paxillin serves to ameliorate both early and late stage progression of ischemic retinopathy - a disease associated with aberrant retinal neovascularization (RNV), the leading cause blindness. Targeting late stage RNV with anti-VEGF protein therapeutics currently serves as the only means of mitigating neovascular eye disease. As such, these treatment modalities do not address the early pathogenesis of ischemic retinopathy (i.e., inflammation), nor do they overcome the long-term challenges of therapeutic resistance due to significant contribution of other pro-angiogenic factors. We therefore explore potential new drug targets using a variety of stressors, confirmation assays and relevant animal models to develop new rational treatment strategies for ischemic retinopathy and neovascular eye disease in humans.

\section{Specific Aim 1}

Angiogenesis is centric to the mitigation of radiation-induced damaged and ischemia. However, the reparative mechanisms that allow for tissue regeneration after total body irradiation are what also contribute to the pathogenesis of neovascular eye disease. We theorized that the mobilization of endothelial progenitor cells (EPCs) depend on integrin and focal adhesion signaling to mitigate radiation injury in total body irradiation (TBI) models. We hypothesized that genetic predisposition to radiation susceptibility and survival is heritable and correlates with gene products that regulate proper mobilization, homing, and adhesion of bone marrow-derived precursor and inflammatory cells to sites of tissue damage. In Aim 1 (Chapter 3), our strategy to test this hypothesis included the examination of the genetic predisposition to high-dose ionizing radiation-induced injury in BXD mice, which served as a genetically complex human "population model". BXD studies allowed us to 1) determined how sequence variation contributes to differential susceptibility to radiation injury, 2) examined gene expression levels as they correlated with radio-sensitivity or -resistance and 3) identified novel targets/pathways to be exploited for development of new antiangiogenic agents.

We identified cell markers, activators and regulators of hematopoietic stem/precursor cell mobilization that correlated with enhanced survival time in BXD mice whose genetic makeup more closely resembled the radioresistant C57BL/6J background. Thus, we hypothesized that inhibition of FA protein paxillin in $\mathrm{C} 57 \mathrm{BL} / 6 \mathrm{~J}$ mice prevents homing and adhesion of mobilized reparative cells to sites of tissue injury and enhances mortality following TBI. We tested this hypothesis using a known paxillin inhibitor, 6-B345TTQ in the TBI model, and confirmed its in vitro activity in a physiological co-culture cellular adhesion assay. 


\section{Specific Aim 2}

Inflammation of and damage to microvessels leads to inflammatory and reparative cell homing and adhesion, which is touted as the primary mechanism of early ischemic injury in both diabetic retinopathy (DR) and radiation retinopathy (RR). Previous work identified KZ-41 as a radiomitigant/protectant by providing a significant survival benefit in the murine TBI model, as well as restoring microvascular flow in combined radiation and vascular injury paradigms $[88,89]$. In an in vitro model of genotoxic stress using the alkylating agent melphalan, we showed KZ-41 specifically counteracts p38 MAPKdependent pro-apoptotic and inflammatory signaling in primary human retinal endothelial cells (REC) [90]. Thus, we hypothesized that KZ-41 prevents ischemic retinopathy through direct modulation of $\mathrm{p} 38$-dependent inflammatory cell adhesion and aberrant proliferation. In Aim 2 (Chapter 4), we tested this hypothesis by delineating the mechanisms of p38-dependent acute radiation injury to human primary RECs exposed to high doses of gamma $\gamma$-irradiation. We used KZ-41 to block p38-dependent signaling in vitro and in the murine oxygen-induced retinopathy (OIR) model of ischemic retinopathy.

\section{Specific Aim 3}

We have identified pathological mechanisms involving p38-mediated regulation and activation of FA protein paxillin, which contributed to both acute injury and ischemia-mediated angiogenesis in the mouse retina. In Aim 3 (Chapter 5), we explicitly target paxillin as an antiangiogenic strategy to mitigate neovascular eye disease. We hypothesized that inhibiting interaction between paxillin and FAK prevents retinal angiogenesis by mechanisms involving the deactivation of the focal adhesion complex (FAC) comprising Src, FAK, and paxillin. In order to test this hypothesis, we developed a novel small-molecule modulator of paxillin signaling, JP-153, and attempt to validate its targeting mechanisms using both in vitro and in vivo models of retinal angiogenesis. We tested target specific interactions between binding partners of paxillin using computational in silico docking protocols.

JP-153 was effective in preventing angiogenesis in the OIR model when administered topically. Therapeutics with antiangiogenic properties, especially those targeting aberrant proliferation have value in their use as systemic therapeutic agents in numerous diseases, i.e., polycystic kidney disease or invasive cancers. Therefore, we developed a selective and sensitive bioanalytical method for the detection of JP-153 in rat plasma (Chapter 6). We characterized the pharmacokinetics with regard to absorption, distribution, metabolism and elimination (ADME) of JP-153 after oral and intravenous administration and performed in vitro liver enzyme metabolism/interaction studies to determine its specific metabolic activity and stability. These efficacies and PK descriptors now serve as the basis for the future development of more potent and more metabolically stable JP-153 analogs for the treatment of ocular and systemic neovascular disease. 


\section{CHAPTER 3. IDENTIFICATION OF CANDITATE GENES THAT CONFER RADIATION INJURY SUSCEPTIBILITY IN BXD MICE}

\section{Introduction}

Vascular endothelial growth factor (VEGF) is the primary driver of angiogenesis and is centric to the pathologies of diabetic retinopathy (DR), cancer and other hyperproliferative diseases. FDA approved anti-VEGF therapeutics are commonly used to treat complications of retinal neovascularization (RNV). Unfortunately, neovascularization in general is not curable and thus requires long-term anti-VEGF therapy. Prospective studies indicate as many as $50 \%$ of patients treated with anti-VEGF agents fail to respond to initial treatments, and those that do, eventually lose efficacy over the years. This decline in efficacy is thought to be due compensatory expression of other growth factors, in addition to the induction of pro-inflammatory cytokines that trigger similar angiogenic signaling cascades $[52,57]$. Thus, there is a critical need to identify new targets for angiogenesis, either in conjunction or as an alternative to anti-VEGF monotherapy

Endothelial progenitor cell (EPC) mobilization from BM and homing to ischemic tissue is a natural reparative processes in response to vascular injury [63, 64]. Vascular injury response mechanisms trigger EPC homing which is regulated by numerous factors, including VEGF, fibroblast growth factor (FGF)-2, platelet-derived growth factor (PDGF), and the angiopoietin family (Ang-1 and Ang-2) [65]. In DR dysfunctional EPC recruitment and aberrant activation is known to be primary mechanisms driving pathological RNV in vivo $[8,91]$. We hypothesized that the same reparative processes governing tissue repair after radiation injury is the very process that leads to visual impairment in neovascular eye disease. Our objective herein, was to decipher potential genetic mechanisms of radiation-induced ischemic injury/repair processes in order to identify and validate novel cellular pathways or targets critical for angiogenesis.

Animal models are crucial to our understanding of the genetic underpinnings of many disease phenotypes. Recombinant inbred (RI) mice, in particular BXDs have been instrumental in mapping polygenic traits specifically by identifying regions of a chromosome that governs each phenotype, or so-called quantitative trait loci (QTL) [92, 93]. BXD (B-by-D) mice were generated from C57ㅌL/6J (C57) and DBA/2J (D2) parental strains and have since been expanded to yield increased precision and statistical power [92, 94]. Figure 3-1 summarizes the breeding scheme. The main utility of BXD strains is in determining how common germline sequence differences of the types found in humans (SNPs, indels, and copy number variants) contribute to differential susceptibility to disease [92]. All BXD strains $(\mathrm{n}=102$, at the time of these studies) have been genotyped at 580,000 SNPs, which means the BXD family is extraordinarily wellcharacterized at the genomic level. We explored genetic predisposition to radiation injury to systematically quantify the baseline liability and susceptibility of young adult BXD mice to high dose radiation in order to identify QTL, or target genes associated with survival phenotype (Figure 3-2). 


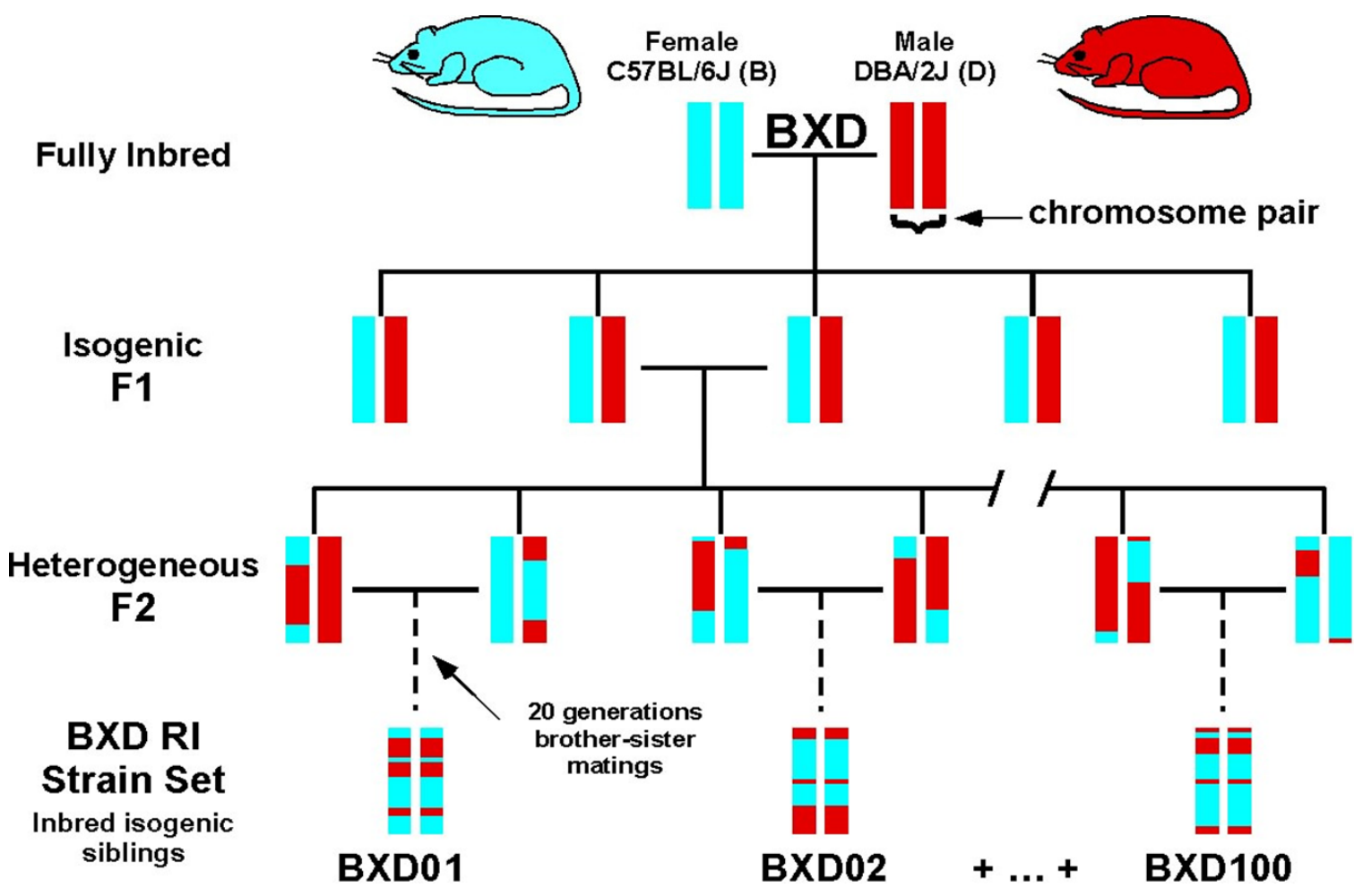

Figure 3-1. BXD recombinant inbred (RI) strain breeding schematic. 


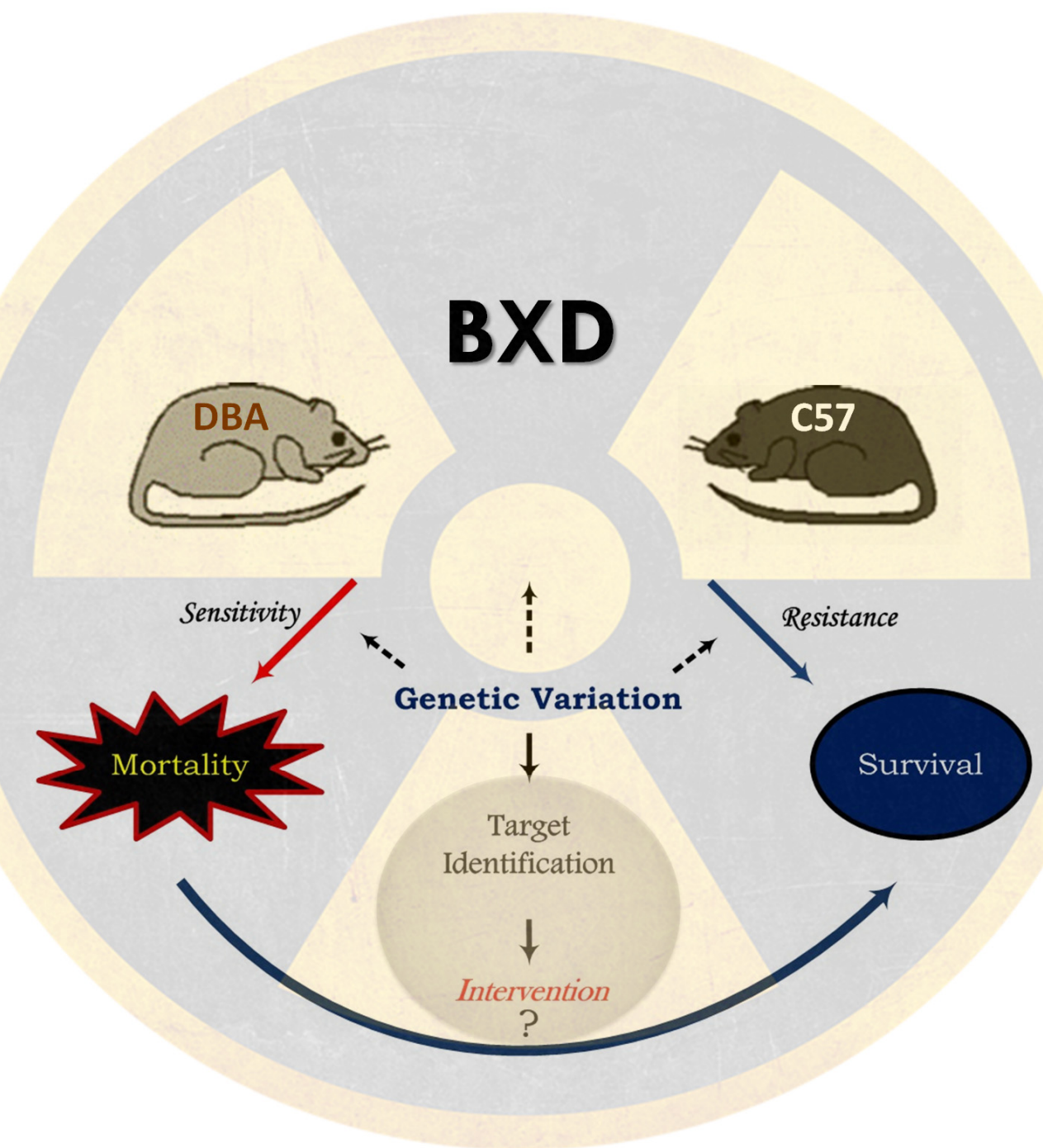

Figure 3-2. The BXD hypothesis.

Conceptual schematic depicting how BXD strains can be used to generate novel candidate target genes/proteins relevant to the genetic predisposition to radiation susceptibility or resistance. 
Since the murine BXD population served as a genetically complex human "population model", this approach enabled us to 1) identify candidate genes/loci underlying radiation injury susceptibility, 2) examine gene expression levels as they correlate with sensitivity or resistance and 3) identify novel targets/pathways for discovery/development of new antiangiogenic therapeutics. Ultimately, this pilot project resulted in the validation of the growth factor/integrin/focal adhesion signaling axis as a target in neovascular disease, and the contributed to the pharmaceutical development of novel paxillin modulator, JP-153 (detailed in Chapter 5).

\section{Materials and Methods}

\section{The murine total-body irradiation model}

All animal experimentation protocols were approved and performed in accordance with the guidelines of the UTHSC Institutional Animal Care and Use Committee (IACUC). All efforts were were made to minimize suffering. Female 12 week old parental strains, C57Bl/6J and DBA/2J were acquired from Jackson Laboratories (Bar Harbor, ME, USA) and two cohorts of 11-13 weeks old female 40 BXD strains $(\mathrm{N}=140)$ were received as a generous gift from Dr. Robert W. Williams (University of Tennessee Health Science Center, Memphis, TN, USA). Animals were housed at UTHSC in a closed-barrier animal facility, $\sim 23.5^{\circ} \mathrm{C}$ and humidity-controlled environment on a $12 \mathrm{~h}$ light-12h dark cycle. Mice were given food and water ad libitum.

The ${ }^{137}$ Cesium radiation source (Mark I Model 25 Gamma Irradiator, J. L. Shepherd \& Associate, San Fernando, CA) produces exposure rates from approximately 1875 to 318 centigray, $\mathrm{cGy} / \mathrm{min}$ according to distance from source (standard practice is to use the lowest dose rate available). The unit is calibrated annually and is maintained by the VA Radiation Safety Officer who conducts weekly functionality and safety checks. We generated mean survival time (MST) data for large cohorts of the $40 \mathrm{BXD}$ and their parental strains. BXD mice (11-13 wks) were irradiated at $6.53 \mathrm{~Gy}\left({ }^{137} \mathrm{Cs}, 2.13 \mathrm{~min}\right)$ to ensure BXD mice were subjected to a single TBI dose known to induce H-ARS. We report and analyze MST ( \pm standard error) as described previously [95]. Animals were minimally manipulated throughout the study period, as even modest stress associated with weighing can significantly perturb MST data. Animals were housed in a closed barrier facility and monitored/tended multiple times daily. Maximum survival period was set at 30 days, and mice resistant at the end of the study were censored and recorded as day-of-death $=30$. MST data were analyzed with corrections for differences in body weight, age, litter size, strain epoch (Taylor series BXDs vs. UTHSC strains) and maternal parity. We mapped both the original data and data corrected by multiple linear regression to remove effects of extraneous variables or noise as described previously [96]. Logistic regressions were used to compare overall 30-day survival among the strains and Kaplan-Meier (K-M) survival curves summarized and visualized survival data by strain. 
12-week $\mathrm{C} 57 \mathrm{Bl} / 6 \mathrm{~J}$ female mice were irradiated according to our TBI model protocol (6.53 Gy, $2.13 \mathrm{~min} ; 5$ mice/group) and dosed subcutaneously with test compounds 6-B345TTQ (16.5 mg/kg), 6-B234TTQ (16.5 mg/kg), or DMSO 0, 24, and 48 hours following radiation exposure. 6-B345TTQ and 6-B234TTQ were acquired from Sigma Aldrich (St. Louis, MO).

\section{Genome-wide interval mapping for MST phenotype}

Phenotype MST was subjected to conventional QTL analysis using simple and composite interval mapping using the GeneNetwork analysis tools (genenetwork.org). Single marker regression was performed across the entire mouse complement of chromosomes at markers typed across BXD strains. A likelihood ratio statistic (LRS) was calculated at each marker comparing the hypothesis that the marker is associated with the phenotype with the null hypothesis that there is no association between marker and phenotype. All QTLs were mapped with a precision of $\pm 10 \mathrm{Mb}$ and a subset (those with Likelihood of the Odds (LOD) $>4$ ) to be mapped with a precision of $\pm 2 \mathrm{Mb}(1.5 \mathrm{LOD}$ confidence interval) [97-99]. Genotypes were then regressed against each trait using the Haley-Knott equations implemented in the WebQTL module of GeneNetwork [100, 101]. Empirical significance thresholds of linkage, either significant or suggestive were determined by at least 5000 permutations [102]. As QTL mapping can be affected by the inclusion of outlier data, we 'winsorized' spurious outliers before mapping protocols were implemented in GeneNewtork [103, 104]. Those strains are depicted as bold in Table 3-1 and not included in the data used to generate the QTL maps in our results. GeneNetwork tools were employed for pair-wise scans and Spearman rank correlation statistical analyses. Furthermore, we correlated phenotypes with microarray expression data from multiple data sets within GeneNetwork: hematopoietic cells and gastrointestinal systems [105-107].

\section{Parallel-plate flow chamber adhesion assay}

Cell adhesion under physiological fluid-shear was investigated using a parallelplate flow chamber and continuous flow-loop (Cytodyne Inc., La Jolla, CA) at a shear stress of 2 dyne $/ \mathrm{cm}^{2}$ as previously described [108, 109]. Briefly, RECs seeded onto AFcoated microscope slides ( 75 x 38mm; Corning Inc., Corning, NY) were grown to confluence and irradiated at $30 \mathrm{~Gy}$. Slides were then placed into the chamber and U937 monocytic cells $\left(2.5 \times 10^{6}\right.$ cells $\left./ \mathrm{mL}\right)$ in RPMI media, containing test compound 6B345TTQ $(10 \mu \mathrm{M})$ were perfused over the REC monolayer. Interacting cells were monitored over two hours using at least six different fields of view and digitally recorded for off-line analysis. Images of adherent cells were obtained using a Nikon Diaphot 300 phase-contrast microscope (Nikon, Melville, NY) equipped with CCD series 68 camera (Dage-MTI, Michigan City, IN). 
Table 3-1. BXD strains used in TBI study.

\begin{tabular}{|c|c|c|c|c|c|c|}
\hline Strain & $\mathbf{N}$ & Age (avg.) & Batch & MST & SD & $b$ \\
\hline BXD1 & 3 & 13.43 & 1 & 13.00 & \pm 2.65 & 1.1780 \\
\hline BXD11 & 2 & 12.86 & 2 & 5.50 & \pm 0.71 & -6.3220 \\
\hline BXD12 & 4 & 13.00 & 1 & 14.50 & \pm 1.29 & 2.6780 \\
\hline BXD22 & 2 & 12.29 & 1 & 4.00 & \pm 0.00 & -7.8220 \\
\hline BXD24 & 4 & 11.86 & 1 & 8.75 & \pm 2.22 & -3.0720 \\
\hline BXD27 & 4 & 13.57 & 2 & 6.50 & \pm 1.00 & -5.3220 \\
\hline BXD29 & 5 & 12.00 & 2 & 11.20 & \pm 3.11 & -0.6221 \\
\hline BXD31 & 3 & 11.86 & 1 & 10.67 & \pm 1.53 & -1.1550 \\
\hline BXD32 & 3 & 12.29 & 2 & 9.67 & \pm 2.52 & -2.1550 \\
\hline BXD34 & 4 & 13.14 & 2 & 5.50 & \pm 1.00 & -6.3220 \\
\hline BXD38 & 5 & 12.00 & 1 & 20.20 & \pm 7.69 & 8.3780 \\
\hline BXD39 & 4 & 12.29 & 2 & 26.00 & $\pm \mathbf{8 . 0 0}$ & 14.1800 \\
\hline BXD40 & 4 & 12.86 & 2 & 13.50 & \pm 3.87 & 1.6780 \\
\hline BXD43 & 4 & 12.00 & 1 & 13.50 & \pm 5.74 & 1.6780 \\
\hline BXD44 & 3 & 11.86 & 1 & 10.67 & \pm 3.79 & -1.1550 \\
\hline BXD45 & 2 & 12.71 & 1 & 8.00 & \pm 1.41 & -3.8220 \\
\hline BXD48 & 3 & 13.86 & 2 & 21.00 & \pm 9.54 & 9.1780 \\
\hline BXD48a (96) & 4 & 12.57 & 2 & 12.50 & \pm 11.03 & 0.9279 \\
\hline BXD51 & 4 & 12.43 & 2 & 5.50 & \pm 1.73 & -6.3220 \\
\hline BXD60 & 4 & 13.57 & 2 & 13.25 & \pm 11.09 & 1.6780 \\
\hline BXD61 & 3 & 12.86 & 2 & 23.00 & \pm 12.12 & 11.1800 \\
\hline BXD62 & 4 & 13.00 & 1 & 8.75 & \pm 0.96 & -3.0720 \\
\hline BXD65 & 3 & 13.00 & 2 & 5.67 & \pm 2.08 & -6.1550 \\
\hline BXD65a (97) & 5 & 11.71 & 1 & 14.80 & \pm 3.03 & 2.9780 \\
\hline BXD68 & 5 & 15.57 & 1 & 9.80 & \pm 2.28 & -2.0220 \\
\hline BXD69 & 3 & 12.71 & 1 & 8.33 & \pm 2.31 & -3.4890 \\
\hline BXD70 & 2 & 13.00 & 2 & 11.00 & \pm 0.00 & -0.8221 \\
\hline BXD73 & 2 & 13.43 & 2 & 11.50 & \pm 0.71 & -0.3221 \\
\hline BXD73a (80) & 3 & 13.43 & 2 & 8.67 & \pm 1.15 & -3.1550 \\
\hline BXD75 & 3 & 13.57 & 2 & 4.33 & \pm 0.58 & -7.4890 \\
\hline BXD77 & 2 & 12.57 & 2 & 29.50 & \pm 0.71 & 15.1800 \\
\hline BXD79 & 3 & 12.71 & 2 & 9.33 & \pm 4.16 & -2.4890 \\
\hline BXD84 & 4 & 15.43 & 1 & 11.25 & \pm 2.50 & -0.5721 \\
\hline BXD85 & 4 & 12.14 & 1 & 6.75 & \pm 0.50 & -5.0720 \\
\hline BXD87 & 5 & 12.43 & 2 & 6.40 & \pm 1.14 & -5.4220 \\
\hline BXD89 & 2 & 12.29 & 2 & 12.00 & \pm 2.83 & 0.1779 \\
\hline BXD99 & 5 & 12.43 & 2 & 26.33 & \pm 6.35 & 14.5100 \\
\hline BXD100 & 5 & 12.00 & 1 & 13.80 & \pm 3.42 & 1.9780 \\
\hline BXD101 & 3 & 12.86 & 1 & 11.67 & \pm 0.58 & -0.1554 \\
\hline BXD102 & 5 & 12.86 & 2 & 8.60 & \pm 2.41 & -3.2220 \\
\hline
\end{tabular}

Notes: $\mathrm{N}=$ animals; Bold: winsorized outlier strain data; MST: mean survival time; SD: standard deviation; $b$ : multiple linear regression coefficients. 


\section{Data recording and statistical analyses}

Data were collected over 30-days and recorded multiple times/day to ensure any animal that died hours after our first-of-the-day health checks were an accurate reflection of day of death. We kept records using simple sorting techniques in Microsoft Excel. Data were then exported into Data Desk version 6.1 (Data Description Inc., Ithaca, NY, USA) for statistical multiple regression analysis and general linear model (GLM) analyses by OLS ANOVA. Statistical bootstrapping, permutation, and correlation analysis was performed through expanded tools within GeneNetwork. Kaplan-Meier survival plots were generated in IBM SPSS Statistics for Windows, version 23 (IBM Corp., Armonk, NY) and survival analysis was performed using standard chi-square analysis to test the equality of survival distributions for the different treatment arms.

\section{Results}

\section{MST data shows differential susceptibility to TBI in BXD mice}

Mean survival time following high dose radiation varies at least two-fold among common inbred strains with a conservative heritability estimate of 0.3 to 0.5 [110]. We measured the radio-susceptibility of the BXD's parental strains, C57BL/6J and DBA/2J and showed that MST varied between the two, thus designating C57 mice as the comparatively more radioresistant strain while D2 the radiosensitive (Figure 3-3). To assess the phenotype susceptibility of mice to high-dose IR, we calculated MST for each animal as a quantitative trait. Table 3-1 lists the 40 BXD strains used in final analyses (n $=140$ mice total), their MST values along with regression analysis, and average age of each strain which were tested in two experimental batches. Winsorized MST (in days) and multiple linear regression data of survival scores were processes in GeneNetwork and results depict notable variability among survival scores measured as days post-IR (Figure 3-4). The distribution of the data as plotted in Figure 3-5, shows the survival scores was not normal but bimodal, the largest of which represented 34 strains that died early, or within the first 16 days. The other six strains survived much later, 20 days and up to the entire 30-day period. Our studies revealed an $\sim 8$-fold genetic variation with an estimated heritability $(\mathrm{h} 2) \cong 0.7$ (MST F-ratio: 4.2982; $\mathrm{p} \leq 0.0001)$ between BXD strains with significant inter-strain variability following TBI, which indicates a strong genetic basis for radiation susceptibility among BXD strains.

\section{Phenotypic correlations, QTL identification, and marker regression analysis}

Using forward genetic methods to study correlates, causes, and consequences of high dose radiation exposure in BXD strains [111], we first examined correlations between phenotypic response (i.e., MST) and genetic markers derived from high-density single nucleotide polymorphism (SNP) mapping of individual BXD strains. In this type of analysis, a significant correlation indicates quantitative trait loci, or QTL as being 


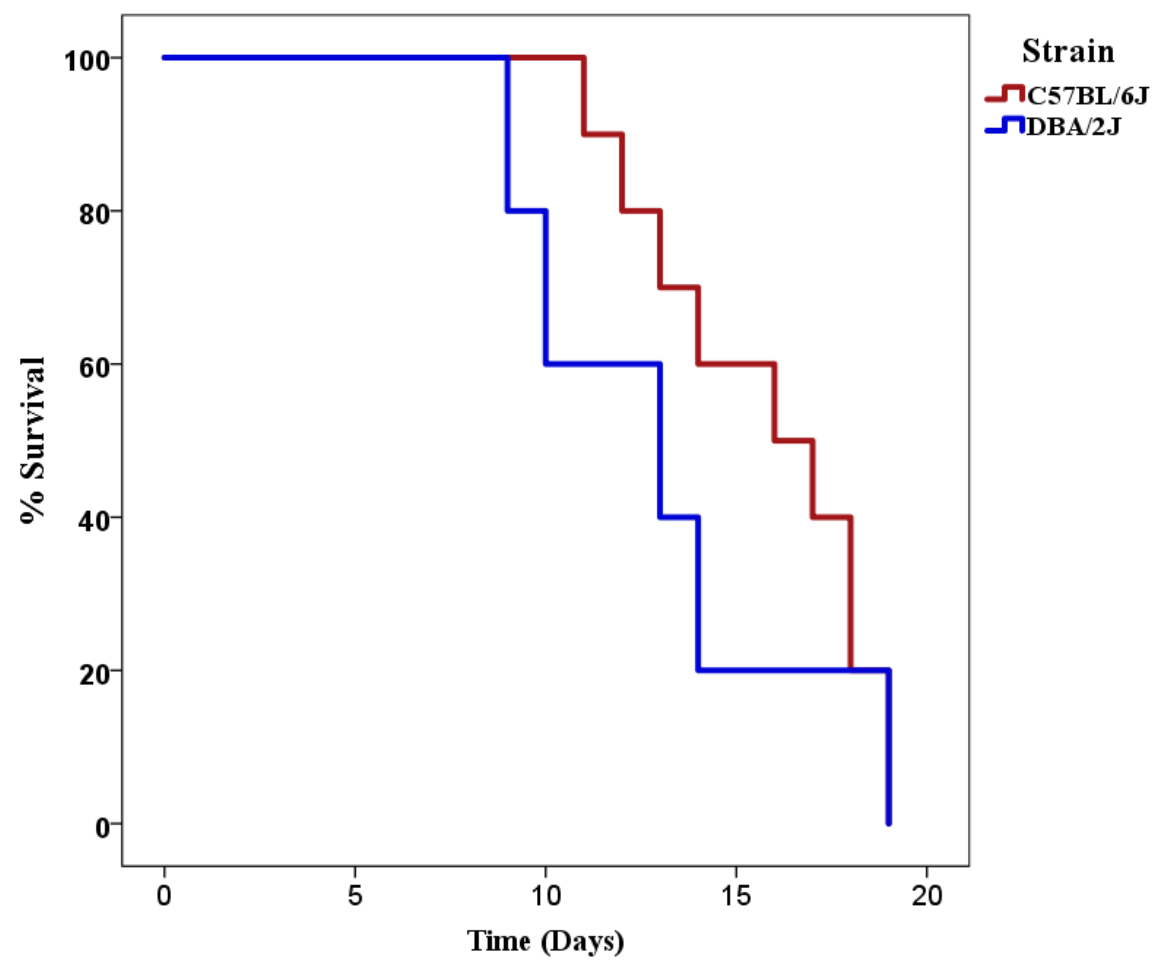

Figure 3-3. K-M survival plot of BXD parental strains C57BL/6J and DBA/2J.

C57 (red/right) and DBA (blue/left) ( $\mathrm{n}=10$ /strain) following high-dose irradiation (6.5 Gy) showed differences in mean survival time. Data were generated in winter of 2009 at UTHSC using animals housed in the Nash vivarium by Yates, Tigyi and colleagues. Animals were not treated with antibiotics. 

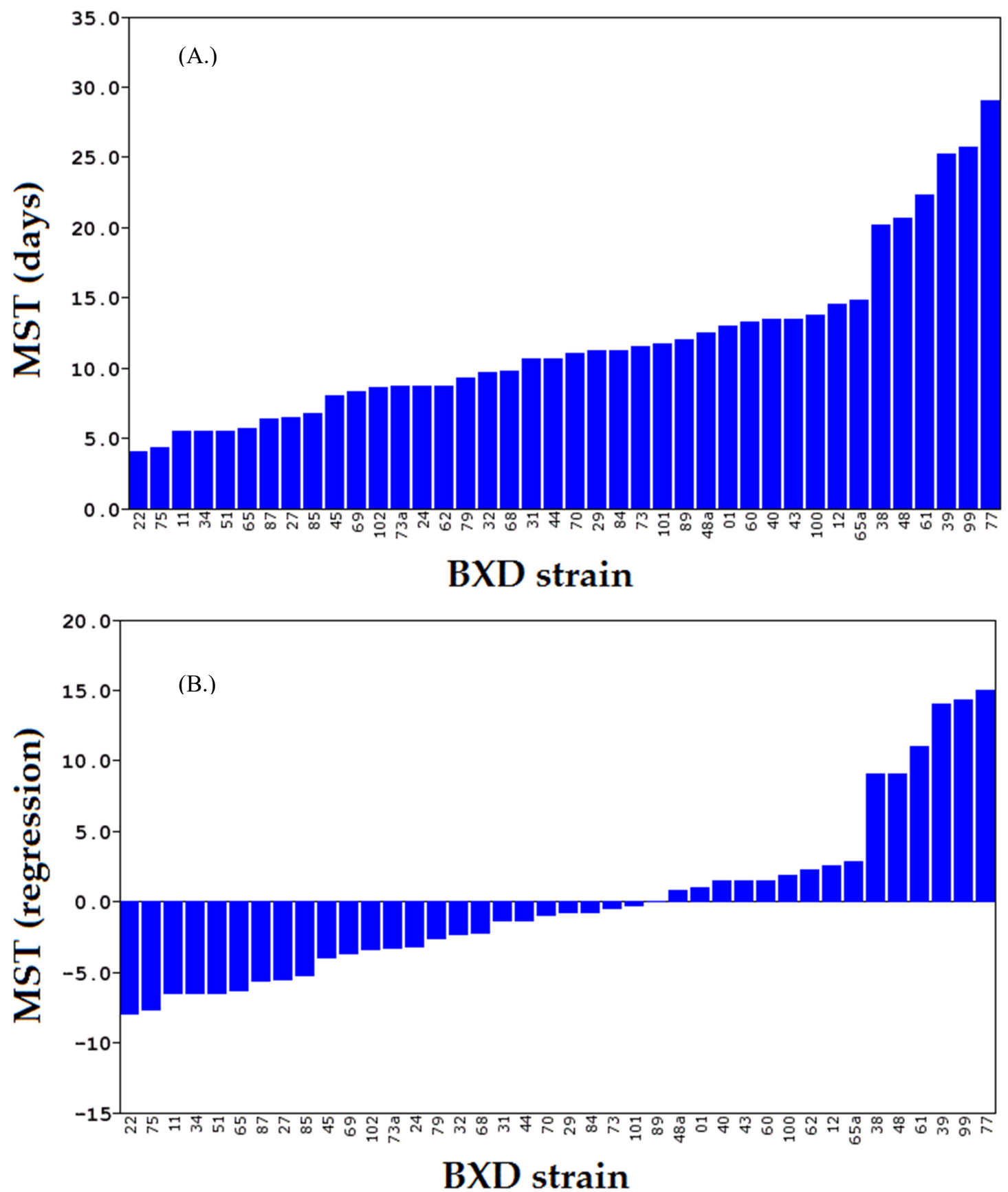

Figure 3-4. BXD mean survival time data.

A) MST, in days of BXD $(n=40)$ following high-dose irradiation. B) Regressed data revealing fold differences among BXD strains, from the most radio-sensitive to the most radio-resistant. 


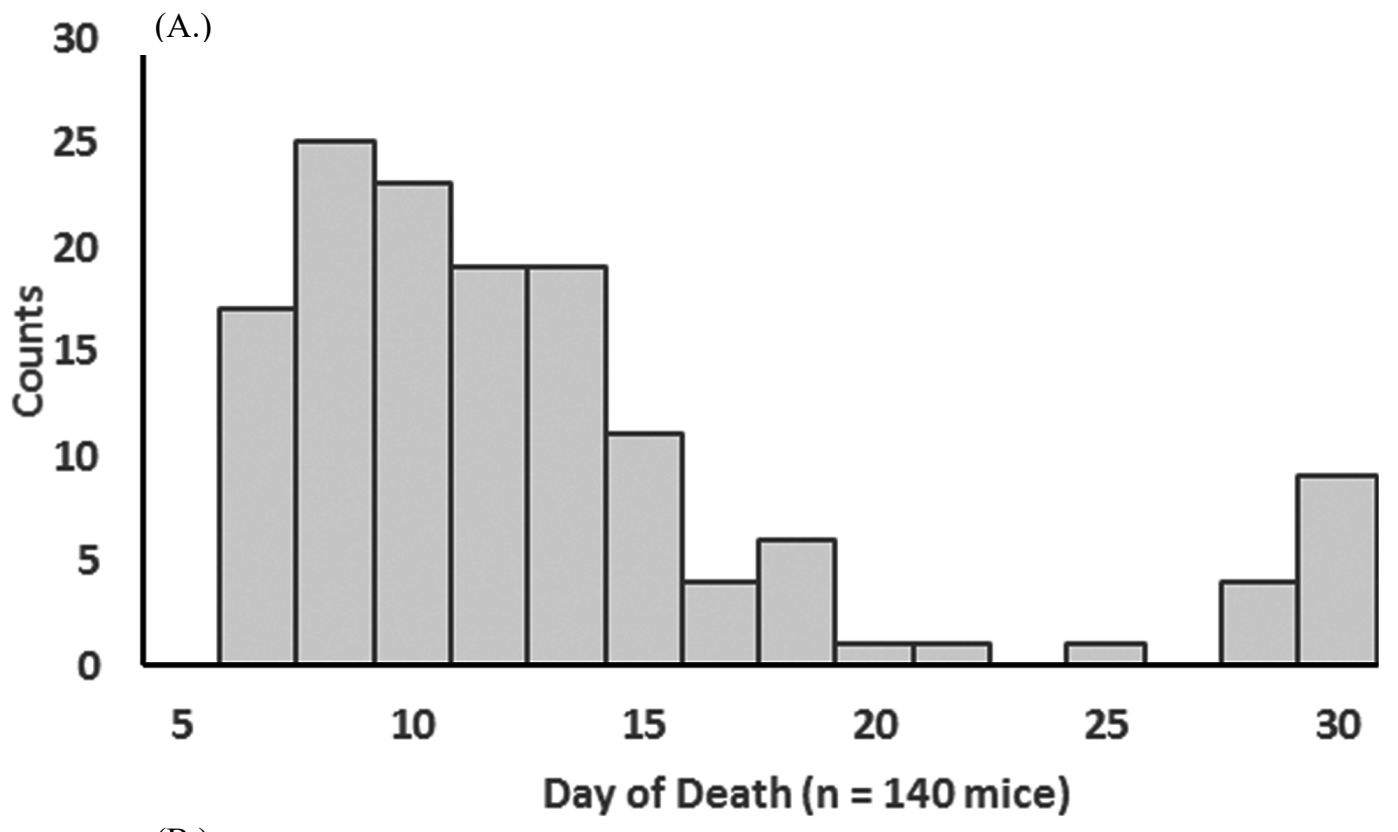

(B.)

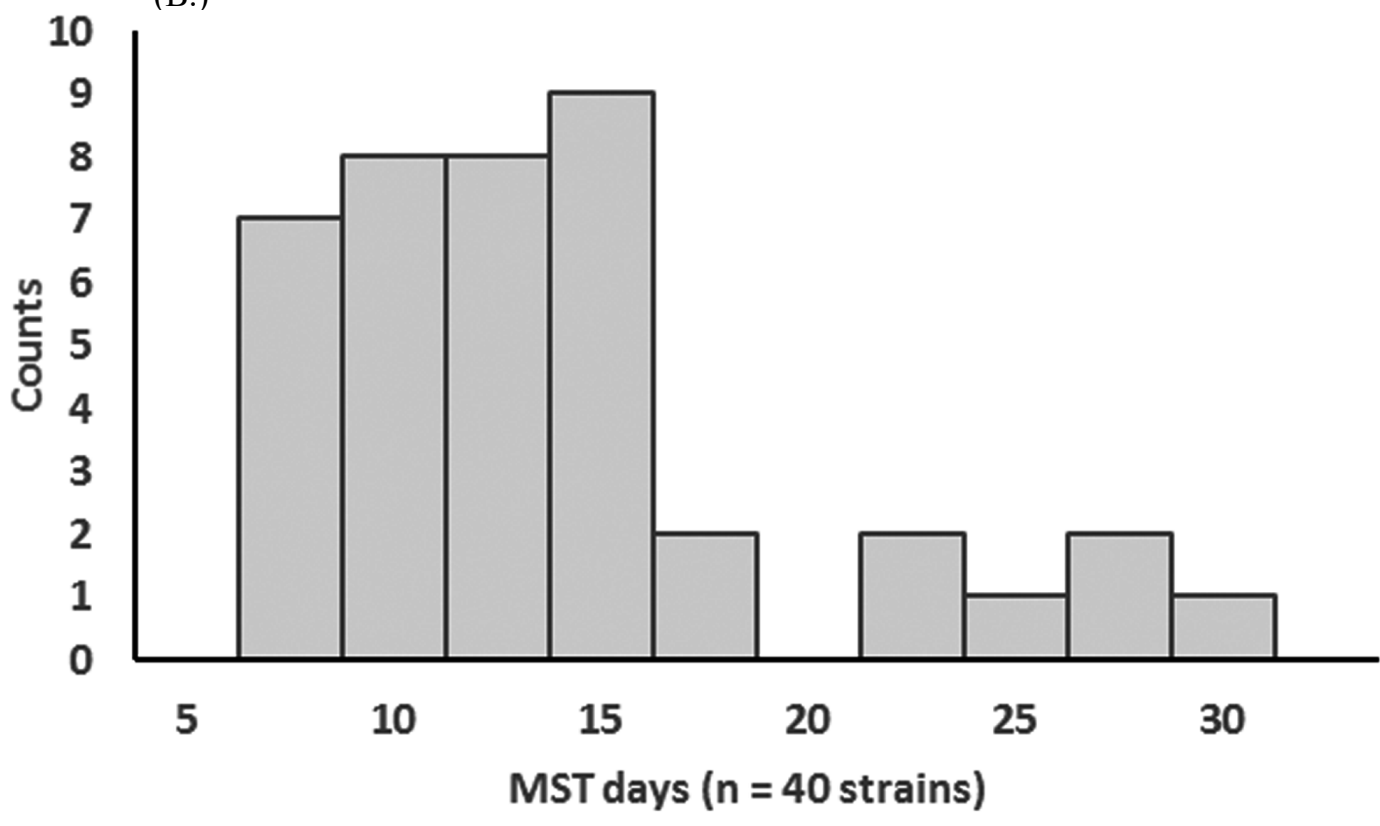

Figure 3-5. BXD survival data distribution.

Histograms showing the frequencies of survival among BXDs exposed to high-dose irradiation. A) Survival time of BXDs, independent of strain grouping, shows a bimodal distribution among 140 mice. B) Histogram of BXD strain MSTs following high-dose irradiation shows data are distributed into two distinct groups, those strains that succumb early post-IR ( $n=34$ strains) and those that are resistant to IR injury ( $n=6$ strains). 
either significant or suggestive in the region where the marker has been mapped. In our current paradigm, a significant QTL threshold was established with a LRS of 16.61, a value statistically determined by GeneNetwork with regard to our specific data set. Through interval mapping, our peaks, as seen in Figure 3-6, reached the suggestive LRS threshold of 10.45. QTL mapping identified several genetic markers with substantial correlation to MST, which are listed in Table 3-2 and Table 3-3 and correspond to peaks located on chromosomes 1 and 5, respectively. The suggestive QTL on chromosome 1 revealed genes associated with cellular anchor biosynthesis pathways, tumor necrosis factor (TNF) subfamily genes, and phosphatases that regulate the activation of Akt and protein kinase $\mathrm{C}$ (PKC) - kinases that are important for cellular survival, proliferation, and angiogenesis. Additionally, a suggestive QTL was identified on a region of chromosome 5 that contained hematopoietic stem cell markers and mobilization through adhesion related genes, inflammatory signaling and pro-angiogenic pathways. Interestingly, many of these genes are associated with wound repair and hypoxic signaling regulation during tissue injury.

QTL marker regression analysis revealed loci on chromosomes 1 and 5, corresponding to the highest LRS scores obtained from MST data (Table 3-4). Marker regressions in GeneNetwork showed the loci within these chromosomes to have an overall "additive effect" of negative proportions, indicating that C57BL/6J alleles increase trait values. Said another way, genetic variation contributing to survival after TBI was closely linked to the genetic background of C57 mice, as negative values correspond to those alleles from C57 mice that contributed to MST phenotypes.

\section{Affymetrix gene correlates reveal associations with genes of both hematopoietic stem cells and gastrointestinal system}

Mechanisms of phosphatase-mediated dephosphorylation, stem cell mobilization, inflammatory and pro-angiogenic pathways were discovered to correlate with, at least in some aspect, to the variation we see in our MST data. Using Affymetrix (oligonucleotide) microarray catalogues [106] we identified correlations in GeneNetwork containing wealth of data on gene expression in hematopoietic stem cells (HSCs), mature progenitor cells, erythroid precursor cells, and myeloid cells, as well as extensive exon-level expression of the gastrointestinal system for a sizable number of BXD strains $(\geq 46)$.

Thus, we are able to leverage GeneNetwork's sophisticated bioinformatics resources to "mine" for interesting genotypes that correlate with variation in MST (Table 3-5).

We found an interesting correlation between our MST data and the gastrointestinal system exons. Gene PPP2R5C, on chr12: 111.812989 which encodes the regulatory subunit of protein phosphatase $2 \mathrm{~A}$ was negatively correlated $(-0.692, \mathrm{P}<$ $0.0001 ; \mathrm{n}=34$ ) with survival in BXD strains. Note the correlation was demonstrated in 34 strains despite our study comprising 40 separate strains. This simply reflects the fact that sequence information at this particular locus is lacking in two strains used in our study. Other important correlations were made with genes associated with cysteine 


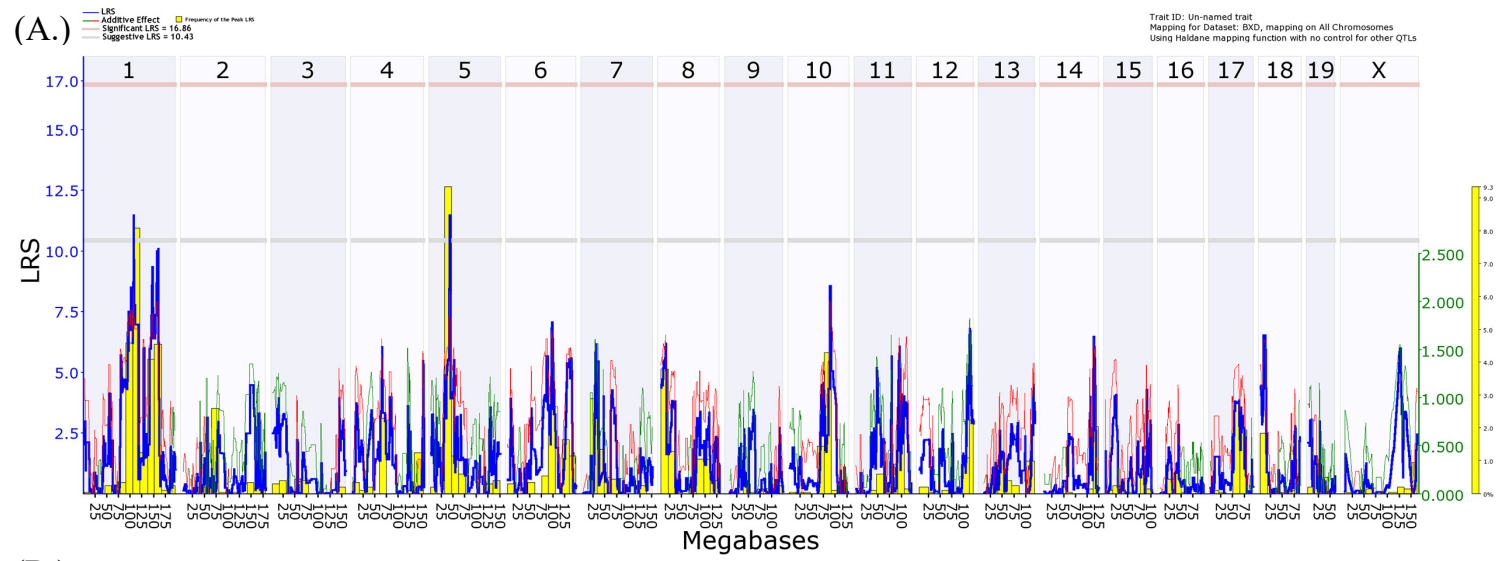

(B.)

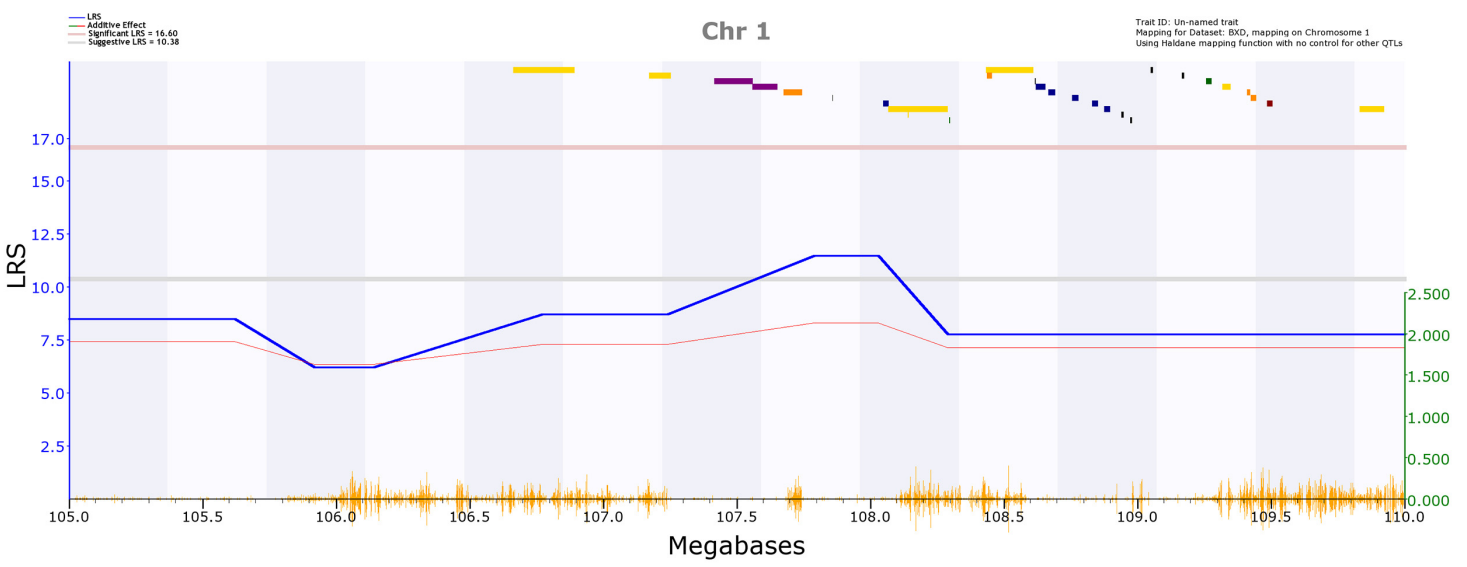

(C.)

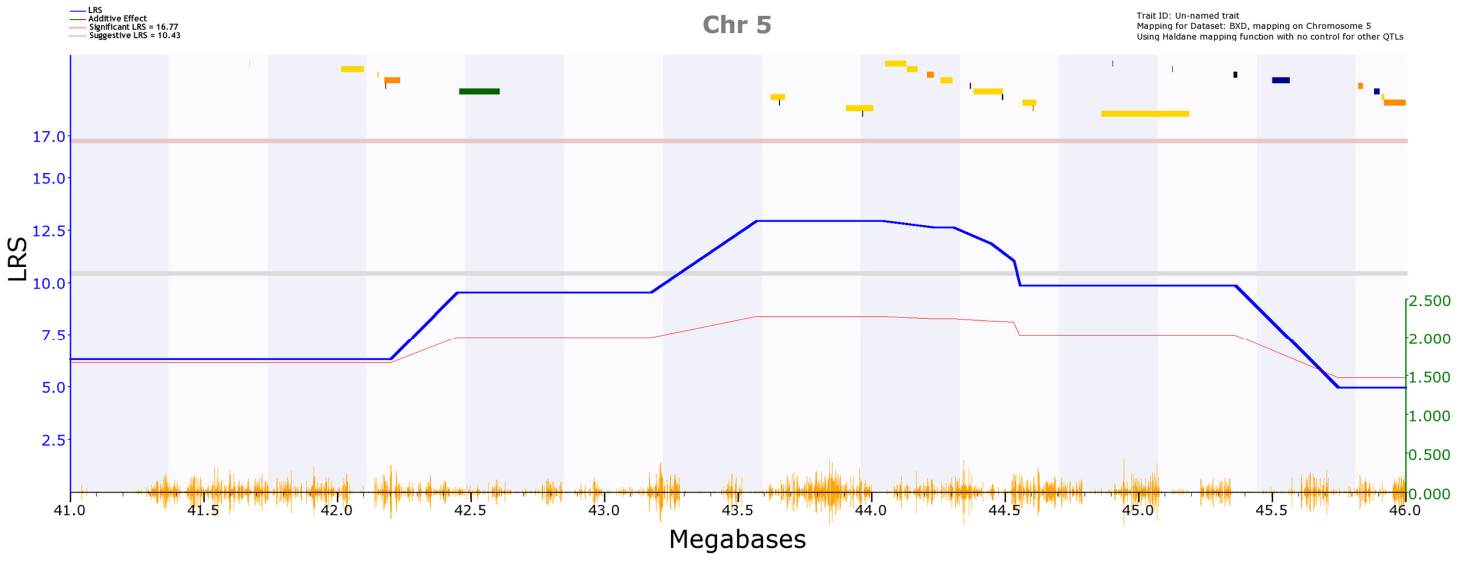

Figure 3-6. Preliminary QTL mapping of MST data among 40 BXD strains.

A) Genome-wide mapping of suggestive QTLs on chromsomes 1 (B) and 5 (C) with highest LRS. Genes for chromosome 1 and 5 (bars at top of each plot) are described in Tables 3-2 and 3-3, respectively. (LRS: blue line; Significant LRS (16.77): top horizontal grey line, Suggestive LRS (10.43): lower horizontal grey line). SNP density depicted as orange "wave" patterns on X-axes. 
Table 3-2. BXD genes associated with suggestive QTL located on chromosome 1.

\begin{tabular}{|c|c|c|c|c|c|c|c|c|}
\hline Gene & Description & Chr & Start & Strand & $\begin{array}{l}\text { Length } \\
\text { (Mb) }\end{array}$ & $\begin{array}{l}\text { Gene } \\
\text { ID }\end{array}$ & SNPs & $\begin{array}{c}\text { SNP } \\
\text { density }\end{array}$ \\
\hline Pign & $\begin{array}{l}\text { phosphatidylinositol glycan anchor } \\
\text { biosynthesis, class N }\end{array}$ & 1 & 107.42 & - & 142.537 & 231207 & 68 & 0.077 \\
\hline Tnfrsfl1a & $\begin{array}{l}\text { Tumor necrosis factor receptor } \\
\text { superfamily, member } 11 \mathrm{a}\end{array}$ & 1 & 107.68 & - & 67.259 & 242960 & 52 & 3.167 \\
\hline Phlpp & $\begin{array}{l}\text { PH domain and leucine rich repeat } \\
\text { protein phosphatase } 1\end{array}$ & 1 & 108.07 & + & 221.135 & 12182 & 71 & 2.501 \\
\hline
\end{tabular}

Notes: Chr: chromosome; Mb: mega base pairs; SNP: Single nucleotide polymorphism

Table 3-3. BXD genes associated with suggestive QTL located on chromosome 5.

\begin{tabular}{|c|c|c|c|c|c|c|c|c|}
\hline Gene & Description & Chr & Start & Strand & $\begin{array}{c}\text { Length } \\
(\mathrm{Mb})\end{array}$ & $\begin{array}{l}\text { Gene } \\
\text { ID }\end{array}$ & SNPs & $\begin{array}{c}\text { SNP } \\
\text { density }\end{array}$ \\
\hline Cpeb2 & $\begin{array}{l}\text { cytoplasmic polyadenylation element } \\
\text { binding protein } 2\end{array}$ & 5 & 43.625 & + & 52.232 & 231207 & 68 & 1.302 \\
\hline Clqtnf7 & C1q tumor necrosis factor related protein 7 & 5 & 43.907 & + & 101.017 & 109323 & 209 & 2.069 \\
\hline Bst1 & Bone marrow stromal cell antigen 1 & 5 & 44.210 & $\mathrm{n} / \mathrm{a}$ & 24.576 & 12182 & 71 & 2.889 \\
\hline Cd38 & CD38 antigen & 5 & 44.260 & - & 43.548 & 12494 & 84 & 1.929 \\
\hline Fgfbpl 1 & Fibroblast growth factor binding protein 1 & 5 & 44.370 & - & 2.903 & 14181 & 12 & 4.134 \\
\hline Prom 1 & Prominin 1 & 5 & 44.389 & - & 108.060 & 19126 & 102 & 0.944 \\
\hline
\end{tabular}

Notes: Chr: chromosome; Mb: mega base pairs; SNP: Single nucleotide polymorphism 
Table 3-4. Marker regression correlation analysis and additive effect.

\begin{tabular}{lcccc}
\hline LRS & Chromosome & Mb & Locus & Additive effect \\
\hline 11.466 & 1 & 107.790531 & rs13476033 & -2.130 \\
11.466 & 1 & 108.029791 & gnf01.105.982 & -2.130 \\
11.477 & 5 & 43.571360 & rs13478212 & -2.105 \\
11.477 & 5 & 44.040633 & rs3023596 & -2.105 \\
11.065 & 5 & 44.234623 & UT_5_42.868696 & -2.062 \\
\hline
\end{tabular}

Notes: LRS: likelihood ratio statistic Mb: mega base pairs; Additive effect:

computational correlation of allele contribution to phenotypic data. An overall negative additive effect indicates the $\mathrm{C} 57 \mathrm{BL} / 6 \mathrm{~J}$ allele is more likely to cause the enhancement of survival (or higher MST values) than DBA/2J. 
Table 3-5. Affymetrix tissue mRNA correlation.

\begin{tabular}{llccccc}
\hline Gene & \multicolumn{1}{c}{ Description } & $\begin{array}{c}\text { Location } \\
(\mathbf{C h r}: \mathbf{M b})\end{array}$ & $\begin{array}{c}\text { N } \\
\text { Cases }\end{array}$ & $\begin{array}{c}\text { Tissue Affy } \\
\text { mRNA }\end{array}$ & Correlation & P value \\
\hline Ctso & Cathepsin O & $3: 81.76$ & 9 & Hematopoietic & -0.9875 & $5.48 \mathrm{e}-10$ \\
Casp4 & $\begin{array}{l}\text { caspase 4, apoptosis-related } \\
\text { cysteine protease }\end{array}$ & $9: 5.33$ & 12 & Hematopoietic & -0.816 & 0.00059 \\
Flot2 & Flotillin 2 & $11: 77.87$ & 32 & Gastrointestinal & -0.659 & $2.07 \mathrm{e}-05$ \\
C4a & Complement component 4A & $17: 34.95$ & 32 & Gastrointestinal & 0.648 & $3.30 \mathrm{e}-05$ \\
PPP2R5C & $\begin{array}{l}\text { protein phosphatase 2, regulatory } \\
\text { subunit B (B56), gamma isoform }\end{array}$ & $12: 111.81$ & 38 & Gastrointestinal & -0.632 & $5.95 \mathrm{e}-05$ \\
& & & & & & \\
\hline
\end{tabular}

Notes: Chr: chromosome; Mb: mega base pairs; SNP: Single nucleotide polymorphism 
proteases (caspase 4 and Cathepsin $\mathrm{O}$ ) in hematopoietic cell lines, inflammatory signaling proteins (complement component $\mathrm{C} 4 \mathrm{a}$ ), growth factor receptor tethering protein linked to signal transduction pathways (Flotillin 2). These correlations between MST data and genomic expression data in both hematopoietic stem cells and the gastrointestinal system suggests a crucial role in regulating survival, proliferation and growth-factor mediated signaling during radiation-induced injury.

\section{Targeting integrin-paxillin in C57BL/6J reduces survival following TBI}

Prom1 (AC133), identified in suggestive QTL on chromosome 5, is a cell-surface molecule whose expression is restricted to bone-marrow derived endothelial progenitor cells, EPCs with long-term repopulating ability [112]. EPCs utilize the $\alpha 4$ integrins as surface adhesion molecules indispensable for embryogenesis, hematopoiesis and immune responses [113, 114]. Inhibiting the $\alpha 4$ integrin enhances mobilization of EPCs from the bone-marrow [115], but prevents reparative cells localization to sites of tissue injury/inflammation [116]. Thus, we hypothesized that the contribution of EPCs to injury sites provided a survival benefit to mice exposed to radiation. To test this hypothesis, we used a known $\alpha 4$-paxillin inhibitor, 6-B345TTQ [117] to block recruitment of reparative cells to sites of inflammation in C57 mice exposed to TBI. Our results were striking, as the survival of mice treated with this compound succumbed to death with an MST of 7.0 ( \pm 0.48$)$ days, compared to $20.6( \pm 1.75)$ of "inactive" 6-B234TTQ and $22.0( \pm 2.12)$ days (Figure 3-7). These highly significant results signify that compound 6-B345TTQ essentially prevented the tissue repair necessary for survival in C57 mice $(\mathrm{P}<0.0001)$. Inactive compound, 6-B234TTQ treated mice MST was not significantly different than vehicle.

\section{6-B345TTQ prevents leukocyte adhesion to irradiated retinal endothelial cells}

We showed blocking integrin-paxillin interaction enhanced mortality in C57 mice following TBI. The $\alpha 4$ integrin-paxillin interaction promote adherence of circulating immune cells, as blocking with 6-B345TTQ in the murine thioglycollate-induced peritonitis model prevented leukocyte recruitment to inflamed tissue [118]. We used the parallel-plate flow chamber to investigate physiological cellular adhesion of U937 leukocytes to an inflamed endothelium over two hours following irradiation. We monitored adherent cells at 30-minute increments and showed that radiation alone caused significant increases in leukocyte attachment to irradiated retinal endothelial cells (RECs) at each time point (Figure 3-8). At the peak of leukocyte adherence (two hours) following radiation, we showed drastically enhanced adherence of circulating cells compared to unirradiated (sham) controls $(2.3 \pm 1.5$ vs. $114.9 \pm 18.3$ adhered cells; $* * \mathrm{P}<$ $0.005)$. Treatment with 6-B345TTQ $(10 \mu \mathrm{M})$ significantly reduced radiation-induced adherence $(6.5 \pm 2.1$ vs. $114.9 \pm 18.3$ adhered cells; \#\# $<0.005)$. 

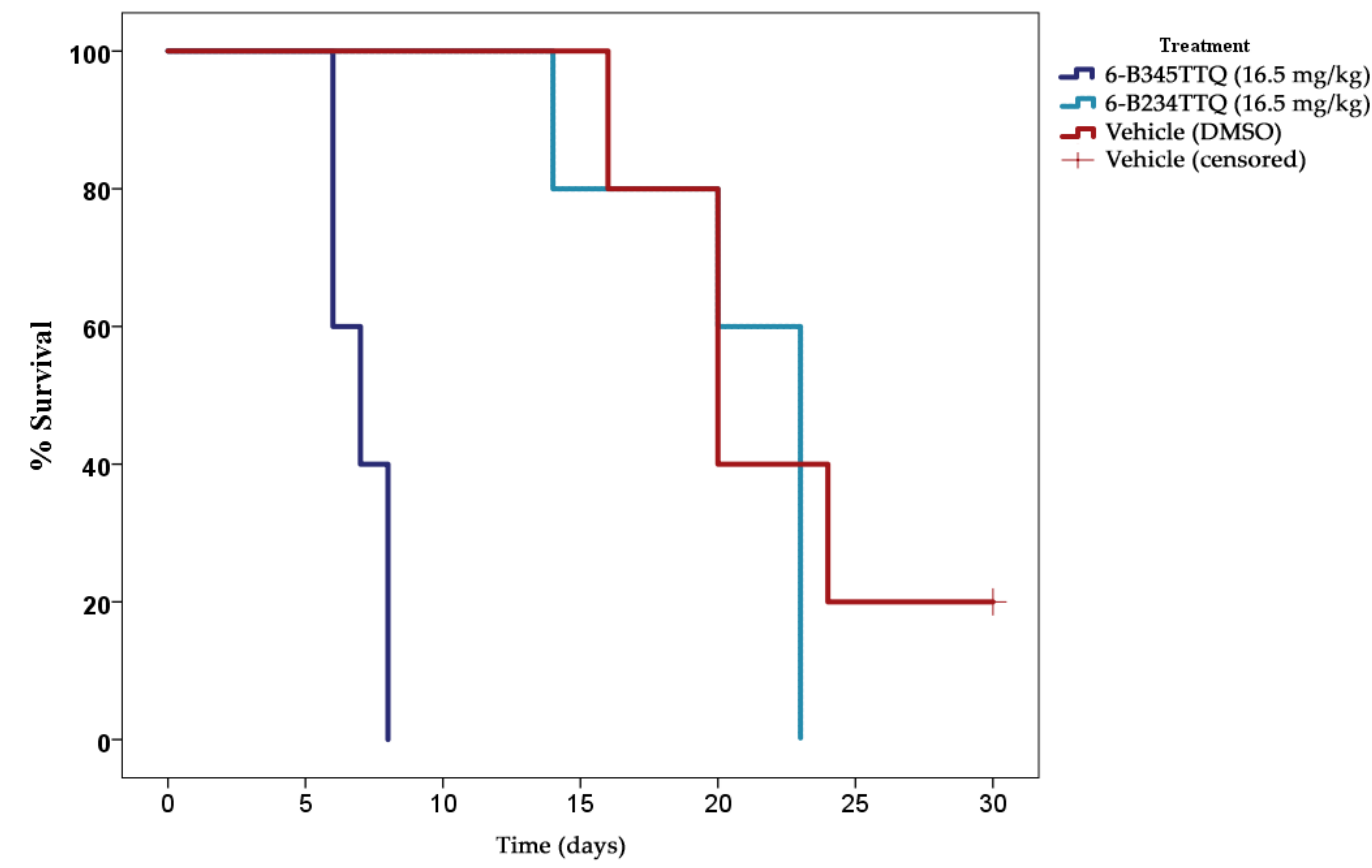

Figure 3-7. K-M survival curves of $\mathrm{C57BI} / 6 \mathrm{~J}$ mice exposed to $6.53 \mathrm{~Gy}$ radiation.

6-B345TTQ (16.5 mg/kg) enhanced mortality of C57BL/6J following high-dose irradiation (6.53 Gy) vs. 6-B234TTQ $(16.5 \mathrm{mg} / \mathrm{kg})$ and vehicle (DMSO) controls $(\mathrm{P}<0.0001)(\mathrm{n}=5 /$ group$)$. 


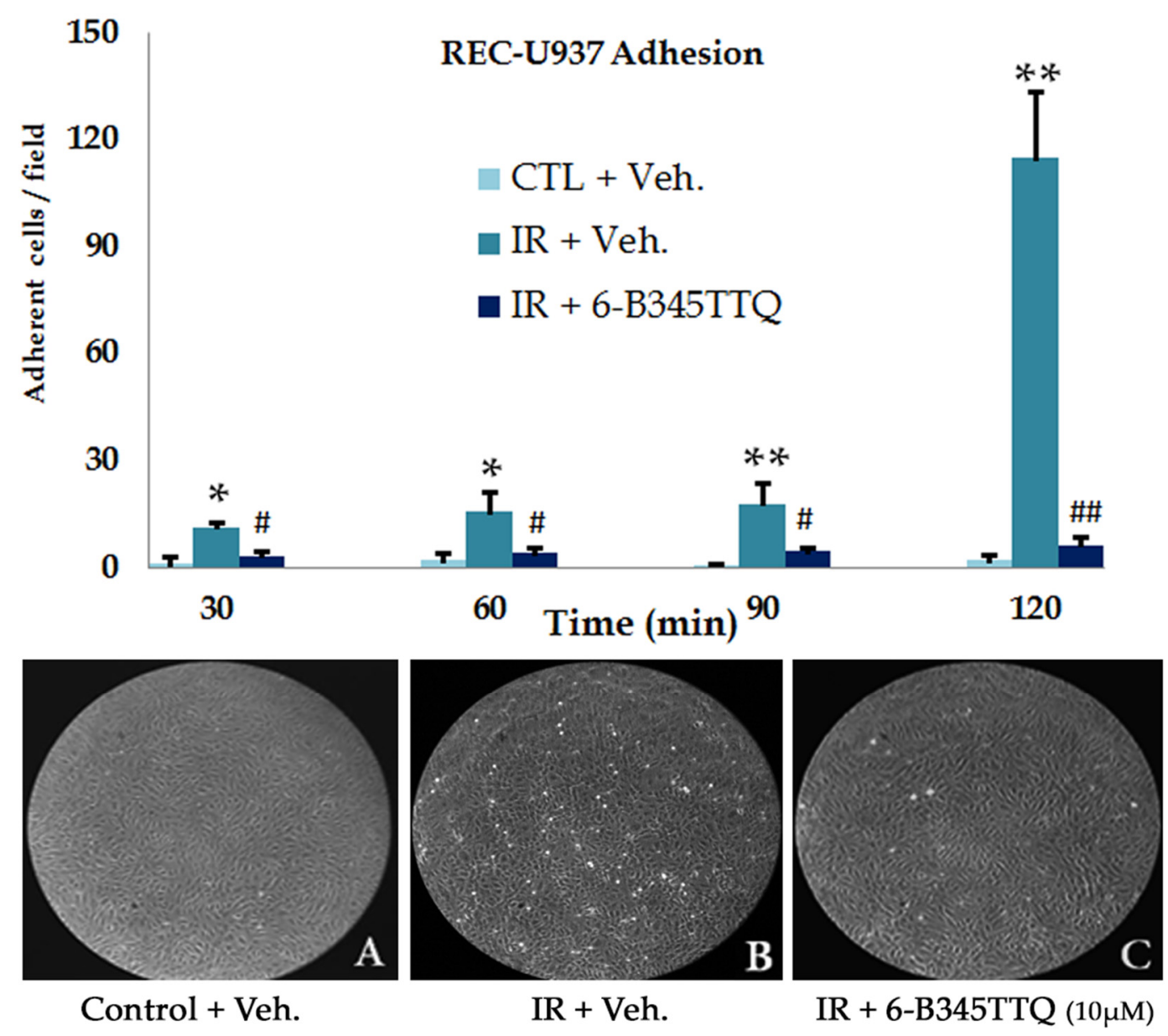

Figure 3-8. 6-B345TTQ reduced adhesion of U937 cells to irradiated endothelium.

Data analysis shows 6-B345TTQ inhibited IR-induced adhesion of U937 cells to inflamed retinal endothelial cell monolayer using the parallel-plate flow chamber $(*, * * \mathrm{P}$ $<0.05,0.005$ vs. un-irradiated controls; \#, \#\# $\mathrm{P}<0.05,0.005$ vs. IR-controls; $\mathrm{n}=$ 6/group). Lower panel shows representative still images taken 1.5 hours post-IR. 


\section{Discussion}

\section{Radiation susceptibility is heritable}

Heritable variation among strains in response to high dose radiation is a finding that goes back to the dawn of the nuclear era. Mean survival time following high dose radiation varies at least two-fold among common inbred strains [110]. Thus, the relevance of the BXD family in our studies was enhanced by the fact that the two parental strains differ in their radiation response. As a result, the normative expression data as it related to the 30-day mean survival time phenotype in irradiated BXD mice showed that 1) radiation injury susceptibility is heritable, and 2) a link exists between MST and sequence variation in genes associated with hematopoietic stem cell markers and angiogenesis-related genes. These suggestive gene loci, or QTLs, linked to MST were revealed on chromosomes 1 and 5, which contained several interesting genetic markers related to stem cell mobilization, endothelial cell integrity, growth-factor mediated tissue repair and angiogenesis.

\section{MST is linked to endothelial stem cells, inflammatory signal regulators and angiogenesis-related genes on chromosomes 1 and 5}

We must emphasize that we have identified suggestive QTLs associated with radiation susceptibility on chromosomes 1 and 5. Too few intra-strain numbers in some BXD strains, with the uncontrollable environmental factors associated with housing and facilities during these experiments, may have impacted our study in such a way that we lacked the necessary parameters to obtain significant QTLs. Nevertheless, a suggestive QTL represents findings that could be of interest to generate hypotheses. We can speculate the potential causality between MST and genotype using these findings, and thus have identified possibilities which link radiosusceptibility with genetic variation. Within these two suggestive QTL peaks, data point to sequence variation in stem cell markers, inflammatory signals, and angiogenesis-related genes with our MST data (Tables 3-2 and 3-3, respectively).

Within chromosome 1, we found genes Pign, Tnfrsf1 la, and Phlpp which correspond to cell surface glycan anchor proteins, a tumor necrosis factor subfamily, and leucine rich phosphatases regulating Akt and protein kinase $\mathrm{C}(\mathrm{PKC})$ isoforms, respectively. SNP density is highest in the latter two genes, while Pign has a very low SNP density measure. This is important because if a locus spans a region with low SNP density, then there is a lower probability that this genetic variant is actually relevant. Tnfrsfl $1 a$ and Phlpp are located within regions of high SNP density, and we can be more certain of their underlying importance. Phlpp encodes the pleckstrin homology (PH) and leucine rich phosphatase family, PHLPP1 and PHLPP2 which are known to regulate the activation and function of Akt and $\mathrm{PKC}$ protein kinases, potent regulators of cell survival [119]. Likewise, TNF receptor subfamily 11a, found in both smooth muscle cells and

endothelial cells, has been shown to be an activator of $\mathrm{NF \kappa B}$, another known regulator of 
cellular survival and proliferation, as well as radiation injury response and tissue repair [120]. Quinic acid derivatives have been suggested to inhibit the activity of NFkB and are potentially useful as anti-inflammatory agents, specifically in providing protective benefits following radiation injury [121]. We further explore this genetic link in more detail in Chapter 4 with KZ-41, a quinic acid derivative known to provide a substantial survival benefit in TBI models [88].

The suggestive QTL mapped to chromosome 5 includes genes, Cpeb2, Clqtnf7, Bst 1, CD38, Fgfbpl, and Prom 1 which all have a basis for immune function, injuryresponse, growth factor-dependent angiogenesis and mechanisms of tissue repair during ischemic injury. The relevance of these genes is not surprising, as radiation elicits an inflammatory and wound repair response in multiple organ systems [122-124].

The Cpeb2 gene encodes a cytoplasmic polyadenylation element binding protein2 , with biological processes related to oxidative stress and hypoxia signaling through translational regulation of hypoxia-inducible factor 1 alpha, $H I F-1 A$, RNA. CPEB2 binds and impedes translation, but under hypoxic stress, CPEB2 dissociated from $H I F-1 A$ RNA allowing for the rapid synthesis of HIF-1 $\alpha$ [125]. Clqtnf7, a gene associated with the C1q TNF superfamily, is a target recognition protein of the classical complement pathway [126]. C1q is known to play a key role in maintaining vascular integrity. In a study investigating lupus pathogenesis, C1q was found to be a mediator of endothelial cell permeability, an important event that is also seen in microvascular complications of radiation retinopathy and diabetic disease [127]. Another related study revealed a direct link between $\mathrm{Clq}$ and integrins in endothelial cells, both working together to promote endothelial cell adhesion and spreading [128]. Bst1 (bone marrow stromal cell antigen-1) gene encodes the ADP-ribosyl cyclase 2 enzyme and also shares 33\% sequence similarity with $C D 38$. The surface marker CD38 is also known as a leukocyte activating antigen, which is involved in host immune response to infectious, tumoral, and metabolic diseases [129]. Bst1 is linked to inflammation-centric rheumatoid arthritis and more recently, was found to be an important gene regulating the immune response in low dose-rate irradiated mouse whole brain, as Bstl enhanced activity of VEGF, Ang (angiopoietin) -1 and -2 in brains irradiated at 10 Gy [130].

The suggestive QTL of chromosome 5 included gene, Fgfbpl with the highest SNP density, and the most relevant to direct tissue repair during ischemic injury. Fibroblast growth factor binding protein 1, a product of $F g f b p l$, is a known regulator of angiogenesis and wound healing and is known to enhance the activity of FGF in neoangiogenesis involving endothelial progenitor cells, fibroblast migration, macrophage recruitment, and epithelial closure, as well as a major driver of injury-mediated pathological disease [131]. FGFBP1 enhances progression and malignancies of certain types of cancer, a pathogenesis that is sometimes referred to as an "overhealing wound" [132]. 


\section{Endothelial progenitor cell recruitment may confer survival benefit of C57}

There is a clear link between ischemic-injury response and the mobilization of stem/progenitor cells into the circulation after vascular injury, a reparative process [133]. EPCs localize to injured tissues to help repair damage through angiogenesis-mediated proliferation and formation of vascular plexus, and contributes to embryogenesis, hematopoiesis, and immune responses [113, 114]. Prom 1 encodes the cell-surface molecule CD133 (AC133) whose expression is restricted to bone-marrow derived EPCs with significant replicative ability [112]. Thus, our identification of gene Prom 1 is not surprising considering the correlations with the above genes responsible for vascular health and repair following ischemic injury.

Residing normally in bone-marrow, EPCs use $\alpha 4$ integrins for mobilization and recruitment to ischemic tissues during the reparative process, which is triggered by numerous cytokine and chemoattractants, including stromal cell-derived factor SDF-1, FGF, and VEGF [133]. Inhibiting $\alpha 4$ enhanced mobilization of EPCs from the bonemarrow [115], but also prevented reparative cells from infiltrating sites of tissue injury/inflammation through specific dysfunction of basic adhesive interactions [116]. We devised a strategy to test our hypothesis using 6-B345TTQ in the setting of TBI, to prevent reparative cells from adhering to and accessing severely damaged tissue in the radioresistant C57 background. Our thought was that if we blocked homing to injured tissues, we could negatively affect survival to "mimic" the DBA radiosensitive phenotype in otherwise radioresistant C57s. We showed that this was in fact the case when mice were treated immediately following radiation exposure, and subsequently for two days afterwards. MST for C57s dropped nearly three-fold with 6-B345TTQ. Interestingly, we used an "inactive" analog, 6-B234TTQ and normal IR resistance was rescued in C57 mice. We further confirmed, in vitro that 6-B345TTQ effectively blocks inflammatory cell adhesion under physiological flow conditions.

\section{Affymetrix mRNA correlations reveal genes associated with an angiogenic- reparative response}

IR damage to specific tissues confer mortality, which is characterized by the syndromes associated with levels of acute radiation exposure, i.e., Heme-ARS or GIARS, affecting the hematopoietic and gastrointestinal system, respectively. Immune cell homing to tissue injury is essential for survival in these models. Focal adhesion complexes comprising paxillin, Src-family kinases, and focal adhesion kinase (FAK) coalesce around integrins to facilitate adhesive interactions with the extracellular matrix or endothelium, as is the case with immune cells. Thus, we explored potential tissuespecific correlations using intricate data analysis tools in GeneNetwork.

Notable Affymetrix tissue mRNA correlations were demonstrated to contain markers associated with both the hematopoietic and gastrointestinal systems, comprising proteolytic, apoptotic and inflammatory markers. Cysteine proteases, caspase 4 and Cathepsin $\mathrm{O}$ are regulators of focal adhesion turnover and protein degradation during 
cellular migration, and inhibits the pro-survival effects of focal adhesion kinase (FAK) through its direct cleavage $[134,135]$. Removing or inhibiting FAK results in disruption in the crucial regulation of endothelial cell-cell contacts and adhesion to the extracellular matrix, a vital component to proper function and integrity of the hematopoietic system [136]. Complement component 4A (C4a) is important in the pathology of early inflammatory stages in age-related macular degeneration, as $\mathrm{C} 4 \mathrm{a}$, along with other complement proteins within this cascade are inflammatory mediators and are involved in multiple immune functions during the disease progression [137]. Additionally, correlations identified a gastrointestinal component gene: protein phosphatase 2 regulatory subunit $B$, gamma (PPP2R2C), mapped to chromosome 12 in mice and chromosome 4 in human. This is an important finding considering the $P P P 2 R 2 C$ encodes the regulatory subunit of protein phosphatase, PP2A which is a known regulator of focal adhesion turnover, cellular growth and division, and is directly linked to the activation of FA proteins, FAK and paxillin which regulate angiogenic responses and can contribute to pathological NV disease [138, 139].

\section{Conclusions and perspectives}

The major achievement of this Aim was the identification of gene loci and a list of high quality candidate genes that were responsible for the MST phenotype. We identified suggestive QTLs that mapped to chromosomes 1 and 5 which contained several interesting markers of ischemic injury and stem cell-centric repair mechanisms. We also were able to cleverly assess the biological components/function of these genes as they related to the phenotypic response in the radioresistant C57BL/6J. Thus, we generated and tested the hypothesis that radiation injury response was mediated by stem cell mobilization and homing during IR-injury repair, and by blocking this activity in vivo and in vitro, we identified lead cellular pathways involving integrin-mediated focal adhesion signaling. Thus, our findings confirm our hypothesis that an inhibitor of radiationinduced repair mechanisms, acting to block the integrin-paxillin interaction, enhanced mortality in C57 mice. In the next chapters, we investigate the merit of our findings herein to make the case for anti-inflammatory/radioprotectant and anti-angiogenic compounds for the treatment of ischemic retinopathy and neovascular disease, respectively. 


\section{CHAPTER 4. MODULATION OF RADIATION INJURY RESPONSE IN RETINAL ENDOTHELIAL CELLS BY QUINIC ACID DERIVATIVE KZ-41*}

\section{Introduction}

In Chapter 3, we identified pro-inflammatory and pro-angiogenic components responsible for radiation injury and tissue repair using the BXD strains exposed to total body irradiation. Our model uncovered genes that are associated with growth factors, inflammatory signaling and activators of neovascularization which ultimately correlated with phenotypic mean survival time data in our model. We aimed to further explore how retinal endothelial cells (REC) specifically respond to irradiation injury, as radiation exposures to the retina can lead to severe vision loss, a condition known as radiation retinopathy $(\mathrm{RR})$.

Development of therapeutics for individuals who receive intended (i.e., radiotherapy) or accidental (i.e., nuclear accident) radiation exposure is a high priority. There are currently no treatment options, before or after, for ameliorating the initial damage associated with radiation injury, i.e., inflammation and ischemic cell death. RR is a progressive disease stemming from radiation-induced capillary injury, similar to the vascular damage seen with diabetic retinopathy $[33,34]$. RR results from exposure of the eye to various directed radiotherapy interventions such as external beam, plaque brachytherapy, and gamma knife [35-37]. Radiotherapy is also used to treat uveal melanoma since it provides both equivalent local tumor control and enhanced survival compared to enucleation (eye removal) $[140,141]$. The incidence of RR in patients with uveal melanoma treated with plaque brachytherapy has been estimated at $20 \%$ with a subset of these patients developing proliferative neovascularization [41-43]. RR results in treatment related visual loss and in cases of severe neovascularization can cause glaucoma, necessitating secondary enucleation for a blind, painful eye [142].

Radiation damage to the vascular retina triggers a pro-inflammatory response characterized by leukostasis, vaso-occlusion, capillary cell death, and subsequent ischemia $[23,24]$. Clinical features of progessive ischemic retinopathy include vascular leakage and capillary non-perfusion, attributed in large part to the accumulation of immune cells in the damaged areas [22]. An ischemic retina triggers subsequent growth factor-mediated neovascularization which results in loss of vision. Studies inhibiting adhesive interactions using antibodies against the intercellular adhesion molecule 1 (ICAM-1) prevented retinal endothelial cell dysfunction, death and subsequent tissue ischemia, which in turn, prevents compensatory retinal neovascularization (Figure 1-1) [16].

\footnotetext{
*Adapted with permission from Toutounchian, J.J., et al., Modulation of radiation injury response in retinal endothelial cells by quinic acid derivative KZ-41 involves p38 MAPK. PLoS One, 2014. 9(6): p. e100210
} 
$\operatorname{Gamma}(\gamma)$ radiation-induced DNA double strand breaks (DSBs) trigger phosphorylation of p38 MAPK (p38) and accumulation of p53 in human endothelial cells. The p38 stress kinase pathway plays an indispensable role in promoting inflammatory responses elicited by DNA damaging stressors such as chemotherapeutics, oxidative stress, and radiation [90]. Furthermore, p38-dependent phosphorylation at serine residues at the $\mathrm{N}$-terminus region of $\mathrm{p} 53$ has been shown to enhance its stability, accumulation and activation [143-146]. Activated p53 binds to its cognate DNA response element and promotes the transcription of inflammatory and apoptotic genes, such as ICAM-1 [144]. Activation of this pathway has been linked to pro-apoptotic signaling and transcriptional events promoting p53-dependent cell cycle arrest, inflammation and/or cell death $[147,148]$. Inhibiting p38 signaling in other cell systems impairs DNA-binding and transcriptional activity of p53 leading to a reduction in both inflammatory and proapoptotic signaling [144, 146, 149].

Previously identified radiomitigant, KZ-41 provided a $\sim 50 \%$ survival benefit following total body irradiation (TBI; LD80/30) and enhanced vascular repair mechanisms in a murine combined radiation and vascular injury model [88, 89]. In an in vitro model of genotoxic stress using the alkylating agent melphalan, we also showed KZ-41 to specifically counteract p38-dependent pro-apoptotic and inflammatory signaling in primary human RECs [90]. In this chapter, we hypothesized that KZ-41 would act to ameliorate radiation-induced inflammation, a causaility in ischemic retinopathy.

\section{Materials and Methods}

\section{Chemicals and reagents}

KZ-41 and internal standard (IS) KZ-39 were synthesized and verified to be $>96 \%$ pure by NMR spectroscopy [121]. Structures of both compounds are depicted in Figure 4-1. Calcein-AM was obtained from BD Biosciences (San Jose, CA). Conjugated ICAM-1 (sc-107 PCPC5) antibody was purchased from Santa Cruz Biotechnology (Santa Cruz, CA). DAPI nuclear stain was obtained from Pierce (Rockford, IL). Phosphorylated (Thr180/Tyr182) p38 antibodies were purchased from R\&D Systems (Minneapolis, MN). Phosphorylated (Ser-15, -33, -37) and total p53, p38, ICAM-1, phosphorylated (Tyr118) and total paxillin, cleaved caspase-3 (Asp175) and GAPDH primary antibodies were acquired from Cell Signaling (Danvers, MA). Alpha-tubulin primary and IRDye 800CW goat anti-rabbit and 680LT goat anti-mouse secondary antibodies were purchased from LI-COR Biotechnology (Lincoln, NE). Non-selective p38 inhibitor, SB202190 was purchased from Tocris Bioscience (Bristol, UK). Anthraquinone compound, DRAQ5 ${ }^{\mathrm{TM}}$ was acquired from Biostatus (Leicestershire, UK). 
KZ-41, MW: 233 g/mol

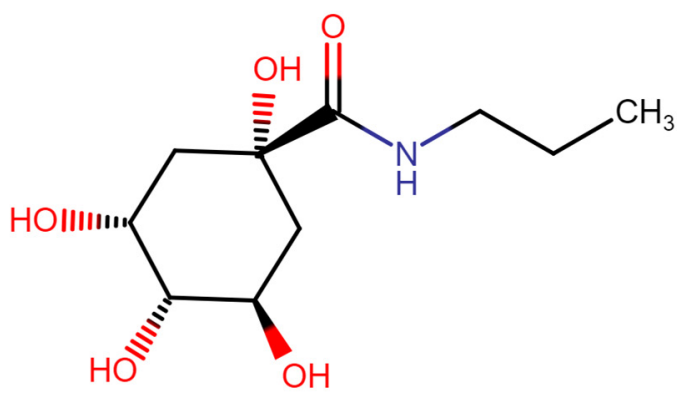

IS (KZ-39), MW: 273 g/mol

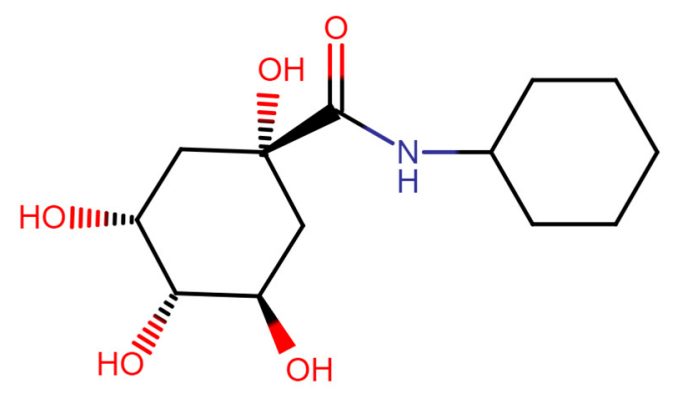

Figure 4-1. Chemical structures of KZ-41 and internal standard (IS) KZ-39. 


\section{Primary cell culture}

Primary human retinal microvascular endothelial cells (RECs, Lot 181) were acquired from Cell Systems Corporation (CSC, Kirkland, Washington). Cells were grown on attachment factor (AF)-coated surfaces in M131 medium with microvascular growth supplements (MVGS), gentamicin $(10 \mathrm{mg} / \mathrm{mL})$, and amphotericin B $(0.25 \mathrm{mg} / \mathrm{mL})$ (Invitrogen; Carlsbad, CA). Only primary cells within passage six were used. U937 (human monocytic-like) cells (ATCC $\AA$ CRL-1593.2 $2^{\mathrm{TM}}$, Manassas, VA) were cultured in RPMI 1640 (Invitrogen) supplemented with 10\% fetal bovine serum, penicillin (5000 IU) and streptomycin $(5 \mathrm{mg} / \mathrm{mL})$. U937 cells to passage 10 were used for adhesion experiments [150]. For immunoassays, RECs were plated into six-well plates and cultured for two days. RECs were pre-treated with KZ-41 $(10 \mu \mathrm{M})$ for 12 hours and then exposed 30 Gy using a Shepherd Mark I, model $68,{ }^{137}$ Cs irradiator (J.L. Shepherd and Associates) at a dose rate of approximately $3 \mathrm{~Gy} / \mathrm{min}$. Non-selective p38 inhibitor SB202190 [151] was added to culture medium 30 minutes prior to irradiation.

\section{Static cellular adhesion assay}

Cellular adhesion under static conditions was assessed using a microplate assay $[152,153]$. Briefly, human primary RECs $\left(10^{5}\right.$ cells/well $)$ were seeded to 96 -well plates and cultured to confluence. RECs were treated with either KZ-41 $(10 \mu \mathrm{M})$ or vehicle (PBS), irradiated (30 Gy), and incubated for 24 hours at $37^{\circ} \mathrm{C}$. Calcein-AM-loaded U937 cells were added to REC-containing wells and allowed to adhere for 30 minutes. Nonadherent cells were removed from wells by gentle washes with (PBS) and adhesion was quantified using a fluorescence microplate reader (excitation/emission wavelengths of $485 / 535 \mathrm{~nm})$.

\section{Parallel-plate flow chamber adhesion assay}

Cell adhesion under physiological fluid-shear was investigated using a parallelplate flow chamber and continuous flow-loop (Cytodyne) at a shear stress of two dyne $/ \mathrm{cm}^{2}$ [108]. Shear stress within the chamber was determined using a constant fluid flow-rate calibrated by adjusting the height of the hydrostatic inlet and outlet ports of the fluid reservoir $[154,155]$. The flow rate for the required shear stress was calculated using the following equation: $S S=6 Q \mu / \mathrm{bh}^{2}$, where $S S=$ shear stress $\left(\right.$ dyne $\left./ \mathrm{cm}^{2}\right), Q=$ flow rate $\left(\mathrm{cm}^{3} / \mathrm{s}\right), \mu=$ fluid viscosity (dyne $\left.{ }^{*} \mathrm{~s} / \mathrm{cm}^{2}\right), b=$ chamber width $(\mathrm{cm}), h=$ chamber height (cm). The schematic of the closed system is depicted in Figure 4-2. RECs were seeded onto AF-coated microscope slides (75 x 38mm; Corning Inc., Corning, NY) and grown to confluence. KZ-41 (10 $\mu \mathrm{M})$ or vehicle-treated RECs were irradiated (30 Gy) and incubated for 24 hours. Slides were placed into the chamber and U937 cells $\left(2.5 \times 10^{6}\right.$ cells $/ \mathrm{mL}$ ) were perfused over the REC monolayer. Interacting cells were monitored over two hours using at least six different fields of view and recorded for off-line analysis. Phase contrast images of adherent cells were obtained using a Nikon Diaphot 300 phasecontrast microscope (Nikon) equipped with a Dage-MTI series 68 camera (Dage-MTI). 


\section{Parallel-Plate Flow Chamber}

Shear stress, $\boldsymbol{\tau}$ is calculated as a

function of measured fluid flow, $Q$ :

$$
\begin{gathered}
\tau=\frac{6 \mu Q}{b h^{2}} \\
\mu=\text { fluid viscosity } \\
b=\text { width of the chamber } \\
h=\text { distance between plates }
\end{gathered}
$$

Figure 4-2. The parallel-plate flow chamber. 
High-resolution video and images were analyzed using Adobe Premier Pro CS5.5 (Adobe Systems). Firm adhesion was defined as interacting cells remaining stationary at the end of two hours $[156,157]$. Data from three separate experiments represent mean adherent cells/fields of view \pm SD.

\section{Immunofluorescence and confocal microscopy}

RECs removed from the flow chamber were fixed in 4\% paraformaldehyde for 15 minutes at room temperature and washed three times with ice-cold PBS. Non-specific blocking of proteins on cellular surface was done using $10 \%$ bovine serum albumin (BSA) containing blocking buffer for at least one hour at room temperature. Human antiICAM-1 antibody conjugated to PerCp-Cy5.5 was diluted in PBS (1:50) and incubated with the slide for one hour at room temperature with gentle rocking. Slides were then washed twice with cold PBS and incubated with DAPI nuclear stain for 10 minutes. Cells were again washed and mounting medium along with cover slips were added to slides and sealed prior to imaging. A Zeiss LSM 710 system with Zen 2010 v.6.0 software (Carl Zeiss Microscopy; Thornwood, NY) was used in image acquisition and analysis.

\section{Immunoblotting and analysis}

Irradiated RECs with or without treatments of either KZ-41 and/or SB202190 were carried out at 30 Gy. For ICAM-1 protein level analysis, REC lysates were collected 24 hours after IR. For phosphorylation status of p38 and p53 stress pathways, REC lysates were collected four hours following exposure to IR. Unirradiated RECs were taken out of the incubator during irradiations for environmental controls. Cellular proteins were analyzed by Western blot after SDS-PAGE using human specific primary antibodies. REC lysates were collected in $1 \mathrm{X}$ RIPA lysis buffer $(50 \mathrm{mM}$ Tris $\cdot \mathrm{HCl}, \mathrm{pH}$ 7.4, $150 \mathrm{mM} \mathrm{NaCl}, 2 \mathrm{mM}$ EDTA, 1\% Nonidet P-40, 0.1\% SDS) with protease and phosphatase inhibitor (1X) cocktail (Roche; Indianapolis, IN). Lysates were kept on ice and centrifuged at $10,000 \mathrm{~g}$ for 10 minutes and cell free lysates were kept at $-80^{\circ} \mathrm{C}$ until further analysis. Total protein concentration was measured by BCA assay (Pierce, Rockford, IL). Protein samples were mixed with 4X LDS loading buffer with 2.5\% 2mercaptoethanol (Sigma), heated to $70^{\circ} \mathrm{C}$ for 10 minutes, and loaded on a NuPAGE 4$12 \%$ Bis-Tris gel (Invitrogen). Immunoblotting was performed with nitrocellulose membranes (Bio-Rad) at 170-mA start and 110-mA end at $25 \mathrm{~V}$ for two hours in NuPAGE transfer buffer (Invitrogen) containing 20\% methanol. Membranes were blocked using Odyssey blocking buffer (LI-COR) for one hour at room temperature with gentle rocking. Membranes were then incubated at $4^{\circ} \mathrm{C}$ with specific primary antibodies (1:1000) overnight. Cellular protein was normalized using GAPDH (Cell Signaling) or $\alpha$ Tubulin (LI-COR) [1:20,000]. Secondary antibodies (IRDye 800CW goat anti-rabbit and IRDye 680LT goat anti-mouse) (LI-COR) [1:10,000] were incubated in the dark at room temperature for 45 minutes. Dual-channel infrared scan and quantitation of immunoblots were conducted using the Odyssey ${ }^{\circledR}$ Sa infrared imaging system with Image Studio (Ver. 3.1.4) (LI-COR). 


\section{Caspase-3 ELISA and ICW activity assays}

The PathScan cleaved caspase-3 (Asp175) sandwich enzyme-linked immunosorbent assay kit (Cell Signaling) was used to evaluate endogenous cleaved caspase-3 levels in REC lysates according to the manufacturer's instructions. For all ELISA analyses, equal protein amounts were loaded into each well, allowing for comparisons using mean optical density (O. D.).

Cleaved caspase-3 levels were measured by In-Cell Western. Briefly, RECs were seeded onto 96-well plates, irradiated and fixed with 4\% PFA following 24-hour incubation in normal tissue culture conditions. Cells were permeabilized using $0.1 \%$ Triton-X100 in PBS for 5 minutes with gentle shaking and repeated for a total of four washes. Odyssey blocking buffer was added to each well and allowed to incubate for one hour at room temperature. Primary cleaved caspase-3 rabbit antibody (Asp 175) in blocking buffer, supplemented with $0.2 \%$ Tween-20 was added to each well and incubated overnight at $4{ }^{\circ} \mathrm{C}$. Wells were subsequently washed four times, and incubated with IRDye $800 \mathrm{CW}$ goat anti-rabbit secondary antibody $[1: 10,000]$ together with DNA counter-stain, DRAQ5 $(0.5 \mu \mathrm{M})$ to normalize for cell number for one hour at room temperature. Intensities of both 700 and $800 \mathrm{~nm}$ channels were measured using the LICOR Odyssey, SA system and quantified according to established system protocols for ICW multi-well plate assays [158].

\section{REC proliferation assays}

To evaluate KZ-41 modulation of irradiation-induced retinal endothelial cell proliferation, 50,000 cells with or without KZ-41 $(10 \mu \mathrm{M})$ were plated into each well of a 96-well dish, irradiated at 30 Gy and incubated for 24 hours. Following treatment with KZ-41 or vehicle, cellular proliferation was determined using the tetrazolium salt WST-1 and a microplate reader (UQuant Reader; BioTek, Winooski, VT) according to the assay manufacturer's instructions (Cell Proliferation Assay Kit, WST dye, ELISA based; Millipore, Billierca, MA) at $450 \mathrm{~nm}$. The absorbance at $450 \mathrm{~nm}$ (recorded as mean OD \pm $\mathrm{SD})$ is directly correlated with cellular proliferative capacity.

\section{Ocular nanoemulsion characterization}

Ocular nanoemulsion (NE) used for drug delivery comprised Capryol 90 (7.5\% $\mathrm{v} / \mathrm{v})$, Triacetin $(7.5 \% \mathrm{v} / \mathrm{v})$, Tween-20 (17.5\% v/v) and Transcutol P $(17.5 \% \mathrm{v} / \mathrm{v})$ (Gattefossé Pharmaceuticals, Saint-Priest, France) generated via homogenization and water titration methods, as previously described $[159,160]$. To examine the physical stability of this system, short- and long-term stability studies were carried out on the ocular nanoemulsion formulation. Samples were divided into three vials after production and stored at room temperature for 60 days. On days $0,7,30$, and 60, all samples were examined and centrifuged for 30 minutes at 13,000 $\times$ g. Turbidity, phase separation, precipitation, drug separation, breaking, and creaming were evaluated, and a translucent formulation was deemed suitable for further testing. Measurement of $\mathrm{pH}$ was carried out 
using the Mettler Toledo $320 \mathrm{pH}$ meter (Mettler-Toledo, LLC; Columbus, OH). Malvern Zetasizer Nano S (Malvern Instruments, Ltd; Worcestershire, UK) was used to determine the main physical characteristics of the nanoemulsion system, including particle size and viscosity. Flow properties were evaluated using dynamic viscosity $(\eta, \mathrm{Pa} / \mathrm{s})$ as a function of time and as a function of shear rate (ranging from $0.1 \mathrm{~s}-1$ to $200 \mathrm{~s}-1$ ). All measurements were performed in triplicate at $24^{\circ} \mathrm{C}$.

\section{Murine oxygen-induced retinopathy model}

All animal experimentation was performed under the guidelines of the Association for Research in Vision and Ophthalmology for the humane use of animals in vision research. The study was approved by the UTHSC Institutional Animal Care and Use Committee (IACUC) in accordance with established guidelines. Eye enucleation was performed under isoflurane anesthesia and all efforts were made to minimize suffering. C57BL/6J (The Jackson Laboratory, Bar Harbor, ME) mouse pups used in these experiments were housed with nursing mothers for the entire study period and given food and water ad libitum.

Retinal neovascularization (RNV) was induced using a mouse model of oxygeninduced retinopathy (OIR) [161, 162]. Mouse pups were randomly divided into four separate groups: 1) Untreated mice under ambient normal oxygen (normoxia) conditions (negative-control); 2) Untreated mice exposed to hyperoxia conditions (positive-control); 3) Nanoemulsion vehicle treated hyperoxia-exposed mice (vehicle-control); and 4) Hyperoxia-exposed mice treated with KZ-41-loaded nanoemulsion (compound-treated). A minimum of five animals were used for each experimental group. C57BL6/J mouse pups were placed in a Plexiglas chamber and exposed to $75 \%$ oxygen maintained and automated by an oxygen controller (Pro-Ox, model P110; Biospherix, Lacona, NY) at post-natal day seven (P7) for five days and then returned to normal oxygen (P12). OIR mice received daily ocular administration of either KZ-41 $(100 \mathrm{mg} / \mathrm{kg})$-loaded nanoemulsion, vehicle (ocular nanoemulsion) or left untreated from P12 to P17. Normoxia (negative controls) mice were not manipulated during the study period. On P17, mice were anesthetized under isoflurane and both eyes were removed, followed by sacrifice. Retinas were harvested, mounted, and stained to investigate retinal angiogenesis $[163,164]$. For retinal whole mounts, enucleated eyes underwent weak fixation (for ease of hyaloid vasculature removal) in $4 \%$ paraformaldehyde (PFA) in PBS for one hour on ice and washed three times. Retinas were then isolated and mounted onto microscope slides. Whole retinas were incubated overnight at $4^{\circ} \mathrm{C}$ with isolectin B4-594 (Alexa Fluor 594; Molecular Probes, Eugene, OR). Isolectin-stained retinas were then washed three times in 1X PBS, sealed on slides using Prolong Gold (Invitrogen), and imaged.

Images were acquired using a Nikon Eclipse 80i confocal microscope and analyzed with Nikon-NIS elements software (Nikon) [165]. Quantification of avascular area (AV) and neovascularization (NV) in retinal whole mounts was performed in Adobe Photoshop (Adobe Systems, Inc.) [163, 164]. Briefly, the AV area was determined by the 
absence of isolectin staining surrounding the optic disc. The area devoid of vascularization was characterized as a percentage of total retinal area (\%AV). Quantification of NV was determined after threshold limits were set within software parameters. This technique ensured the quantification of only clusters and tufts of NV while excluding the normal vascularized retina (less intense staining). Photoshop analysis tools were used to manually outline $\mathrm{NV}$ formations and data was recorded as a percentage of total retinal area $(\% \mathrm{NV})[163,164]$.

\section{KZ-41 ocular pharmacokinetics study in mice}

Murine ocular pharmacokinetics of KZ-41 delivered topically to the mouse eye was characterized for penetration into ocular tissues using analytical methods described previously $[166,167]$. Adult C57BL/6J mice received a single ocular administration of KZ-41-loaded NE $(100 \mathrm{mg} / \mathrm{kg})$ to the right eye; the left eye was dosed with vehicle for contralateral controls. Blood and eyes were harvested from mice ( $\mathrm{n}=3 /$ time-point) over 24 hours at regular intervals of 5, 15, 30, 60, 240, 480, and 1440 minutes. KZ-41 concentration, relative to the internal standard was determined in plasma and eye homogenates by LC-MS/MS. Pharmacokinetic parameters were derived from plasma and ocular tissue concentration vs. time curves using Phoenix WinNonlin version 6.3 (Pharsight Corp., Cary, NC, USA).

\section{LC-MS/MS parameters}

The LC-MS/MS system comprised an API 4500 Q TRAP mass spectrometer equipped with an electrospray ion source (Applied Biosystems Sciex, Foster City, CA) operated in negative-ion mode was used for MS detection. Quantitation was performed using MRM mode to study parent $\rightarrow$ product ion transitions for KZ-41 $(232 \rightarrow 178)$ and IS $(272 \rightarrow 218)$ with unit resolution. Source dependent parameters optimized were gas 1 (nebulizer gas): $30 \mathrm{psi}$, gas 2 (heater gas): $40 \mathrm{psi}$, ion spray voltage (ISV): $-4500 \mathrm{~V}$, temperature: $500^{\circ} \mathrm{C}$. KZ-41 and IS dependent parameters were declustering potential (-50 $\mathrm{V},-60 \mathrm{~V})$, entrance potential $(-10 \mathrm{~V},-10 \mathrm{~V})$, and collision energy $(-28 \mathrm{~V},-32 \mathrm{~V})$, respectively. Cell exit potential $(-15 \mathrm{~V})$ and focusing potential $(400 \mathrm{~V})$ were the same for both KZ-41 and IS. Nitrogen was used as collision-activated dissociation (CAD) gas and was set at 6 . Quadrupole 1 and quadrupole 3 were maintained at unit resolution and dwell time was set at $250 \mathrm{~ms}$ for each MRM transition. Chromatographic separation was performed on a PolyHYDROXYETHYL A ${ }^{\text {TM }}$ HILIC column $(5 \mu \mathrm{m}, 100$ x $2.1 \mathrm{~mm}, 100$ $\AA$, The Nest Group, Inc., Southborough, MA, USA) held at $25^{\circ} \mathrm{C}$. The mobile phase consisted of eluent A (water) and eluent $\mathrm{B}(\mathrm{ACN})$, and separation achieved using a gradient program of 0 min: $15 \% \mathrm{~A} ; 4 \mathrm{~min}: 40 \% \mathrm{~A} ; 4.5 \mathrm{~min}: 15 \% \mathrm{~A}$, at a flow rate of 0.2 $\mathrm{mL} / \mathrm{min}$ performed with a run time of $5.5 \mathrm{~min}$. Analytical data were processed using the software program Analyst (Version 1.6.1). 


\section{Preparation of calibration standards and quality control samples}

KZ-41 and IS stock solutions $(1 \mathrm{mg} / \mathrm{mL})$ were prepared in high purity water. Working solutions of KZ-41 were prepared by further diluting the stock solution in water: acetonitrile $(1: 1 \mathrm{v} / \mathrm{v})$. Non-treated contralateral eye homogenates were prepared in normal saline (1:3, wt/vol) and supernatant was used as both blank controls and for KZ41 calibration standards and QC samples. Calibration standards and QC samples were prepared by mixing $10 \mu \mathrm{L}$ working solution in $90 \mu \mathrm{L}$ drug free vitreous/eye homogenate or plasma, resulting in matrix concentrations of $2.5-500 \mathrm{ng} / \mathrm{mL}$. IS were prepared in precipitation solution to a concentration of $200 \mathrm{ng} / \mathrm{mL}$. Blank and zero samples were prepared using blank plasma and vitreous/eye homogenates. All samples were stored at $80^{\circ} \mathrm{C}$ prior to analysis.

\section{Sample preparation}

Protein precipitation was used to extract KZ-41 (and internal standard) from mouse plasma and eye homogenates. Plasma aliquots $(50 \mu \mathrm{L})$ were added in precipitation solution (ACN, 1:3 v:v), vortexed for 30 seconds, and then centrifuged for 10 minutes at $4^{\circ} \mathrm{C}(12,000 \mathrm{~g})$. The supernatant was transferred to a 96 -well plate and an aliquot $(5 \mu \mathrm{L})$ was injected onto the LC-MS/MS system. Enucleated eyes were rinsed in PBS at least three times to remove any residual compound. Eye homogenate was then prepared in PBS (one eye/ 2 mL PBS) using a soft-tissue homogenizer. Crude homogenate aliquots were added to precipitation solution and vortexed for 30 seconds, centrifuged for 10 minutes $(12,000 \mathrm{~g})$ at $4^{\circ} \mathrm{C}$, upon which supernatant was collected and transferred to 96well plate and injected ( $5 \mathrm{uL} /$ sample) onto the LC-MS/MS system.

\section{LC-MS/MS method validation}

Method validation was conducted in accordance with the criteria suggested by the US Food and Drug Administration (FDA) Guidance for Industry - Bioanalytical Method Validation [168]. Calibration curves were constructed by plotting the peak area ratio of KZ-41: IS against the nominal concentration of calibration standards. For each batch of samples, a calibration curve was constructed from the results of duplicate measurement of 6 concentrations of KZ-41. The peak area ratios of KZ-41 to IS versus corresponding concentrations were used for the linear least-squares regression of the calibration lines and for determination of slopes (A), intercepts (B), and correlation coefficients. Unknown sample concentrations of KZ-41 were calculated from the linear regression equation with a weighted factor of $1 / x 2$. The lower limit of quantification (LLOQ) was defined as the lowest concentration with a signal to noise $>5$ over six independent runs with coefficient of variation $\%$ accuracy within $20 \%$ and precision within $20 \%$ for every run. The limit of detection (LOD) was not determined. Three quality control (QC) validation samples were prepared at nominal concentrations of $2.5,25,50,125,250$, and $500 \mathrm{ng} / \mathrm{mL}$ on the same day. All chromatograms were processed using the automatic integration software module in Analyst 1.6.1 followed by manual check to confirm proper integration of each peak 
area. Each analytical run consisted of a matrix blank, a set of calibration standards, six replicate LLOQ samples, and a set of three levels of QC samples. The acceptance criteria of the data included precision within $15 \%$ relative standard deviation and accuracy within $\pm 15 \%$ deviation of the nominal value. Ion suppression via matrix effect was evaluated by adding three level KZ-41 QC sample with and without IS to extracted blank plasma comparing KZ-41 peak areas to neat solution samples.

\section{Statistical analyses}

Data represented herein were performed in replicates of three or more and presented as the mean \pm standard deviation (SD), unless otherwise indicated. Differences among groups were analyzed using one-way analysis of variance (ANOVA). When overall analysis revealed significance among groups, means were compared and tested using Tukey's post-hoc analysis. Statistical significance was set at $\mathrm{P}<0.05$.

\section{Results}

\section{Radiation induces adhesion of $\mathrm{U} 937$ cells under static and dynamic flow conditions}

A key mechanism of radiation-induced retinal injury involves leukocyte entrapment and accumulation within microvascular circulation $[14,15]$. We used a fluorescence-based static-adhesion assay to determine whether or not radiation induced an adhesive phenotype in RECs (Figure 4-3A). Adhesion of U937 monocytic cells was enhanced by nearly $70 \%$ in irradiated RECs $(* \mathrm{P}<0.005)$. Addition of KZ-41

immediately prior to radiation led to a substantial decrease in adherent cells $(\# \mathrm{P}<0.005)$.

Immunoglobin superfamily of cellular adhesion molecules (e.g., ICAM, VCAM, PECAM, etc.) and the selectin family (i.e., L-, P- and E-selectin) both facilitate comparable adhesion dynamics in static environments $[14,18]$. Therefore, we used a parallel-plate flow chamber and adapted a continuous flow-loop system to monitor, observe, and quantify the three characteristic events of leukocyte attachment in blood vessels: rolling, tethering, and firm adhesion [169]. 24 hours post-radiation, RECs were placed in the flow chamber and U937 cells were perfused over the monolayer for two hours. Adherence was monitored via phase-contrast microscopy and digitally recorded for offline analysis (Figure 4-3B-C). U937 cell adhesion to the REC monolayer was significant enhanced after radiation compared to unirradiated RECs $(2 \pm 2$ vs. $87 \pm 18$ adhered cells; *P < 0.05). Pre-treatment with KZ-41 significantly reduced U937 adherence $(25 \pm 12$ vs. $87 \pm 18$ adhered cells; $\# \mathrm{P}<0.05)$. 

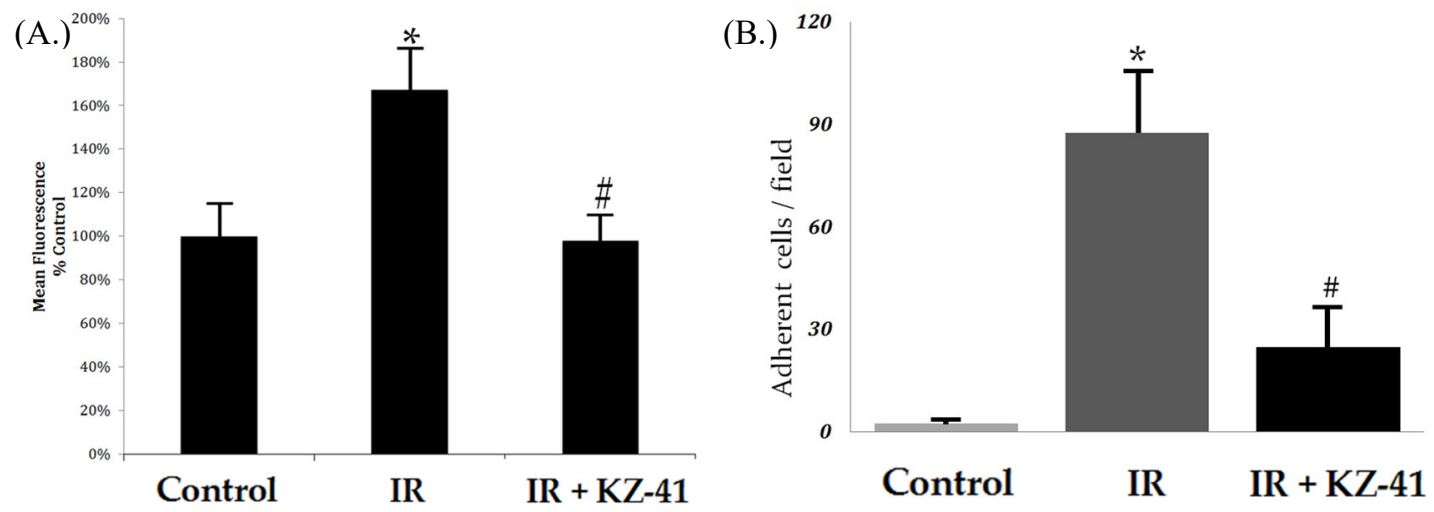

(C.)

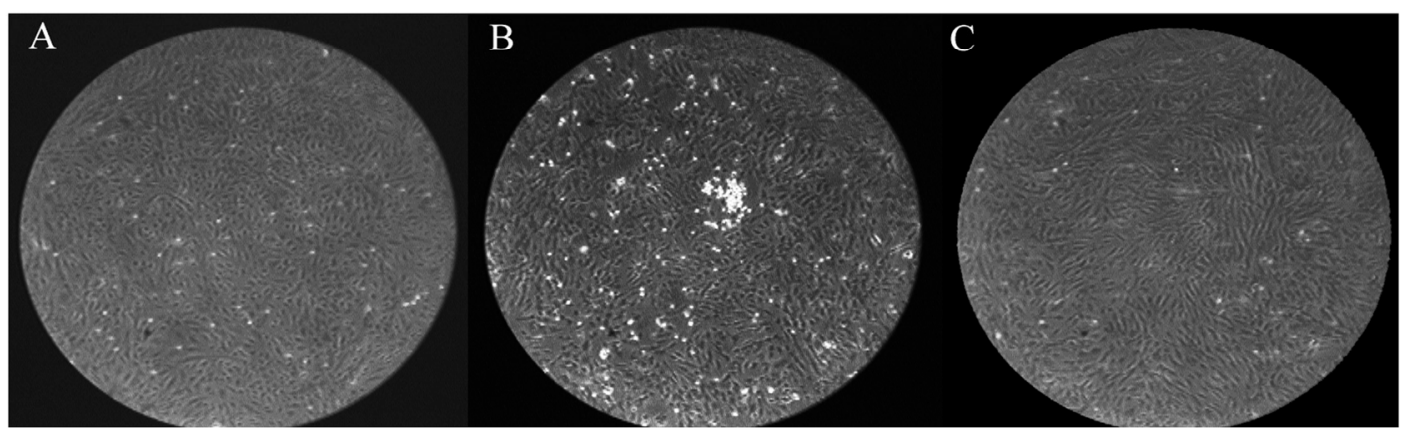

Figure 4-3. Radiation-induced U937-REC adhesion inhibited by KZ-41.

A) Static-adhesion: Irradiated human RECs were incubated for 24 hours in culture medium containing vehicle (PBS) or KZ-41 $(10 \mu \mathrm{M})$. Calcein-AM loaded U937 cells were co-cultured with RECs ( $\mathrm{n}=8 /$ group) for 30 minutes and non-adherent cells were washed from wells; attached U937 cells were quantified with a fluorescence microplate reader (excitation/emission wavelengths of 485/535 nm). Data demonstrate that KZ-41 inhibits IR-induced leukocyte attachment to RECs $(*, * * \mathrm{P}<0.005$ ) (All data normalized to background fluorescence; data represent $\%$ Control fluorescence signal $\pm \mathrm{SD}$ ). B) Flow-Chamber adhesion: RECs were irradiated and cultured for 24 hours. U937 cells were perfused over RECs placed in flow-chamber and digital images were collected after two hours. Data represent mean \#-adherent cells/field \pm SD. KZ-41 $(10 \mu \mathrm{M})$ treatment significantly decreased IR-induced adhesion of U937 cells $(*$, \# P $<0.05)$. C) Representative still images A: Control, B: IR-REC and C: IR+KZ-41 show extent of U937 accumulation on surface of RECs. 


\section{Radiation enhances ICAM-1 and sICAM-1 levels in RECs}

Numerous in vitro and in vivo models of IR-induced vascular injury have established ICAM-1 as an important pathological indicator of inflammation $[14,19,170]$. Moreover, proteolytic cleavage of ICAM-1 from activated endothelium yields so-called shed or soluble ICAM-1 (sICAM-1), which peaks at 24 hours post-IR and correlates with higher total ICAM-1 levels [171]. We confirmed sICAM-1 was enhanced after IR as measured by ELISA from REC media (Figure 4-4; *P <0.05). Similarly, in REC lysates, total ICAM-1 levels were significantly enhanced by radiation (Figure 4-5A; $* \mathrm{P}<0.05$ vs. unirradiated RECs) and were reduced with treatment of KZ-41 compared to irradiated RECs $(* * \mathrm{P}<0.05)$. Surface ICAM-1 protein was also measured in RECs from flowchamber experiments by confocal microscopy and results showed IR-induced ICAM-1 levels are modulated by KZ-41 treatment (Figure 4-5B).

\section{KZ-41 inhibits radiation-induced ICAM-1 expression through a p38 MAPK- dependent mechanism}

In response to DNA damaging events (e.g., radiation), the stress kinase p38 is activated by dual phosphorylation at Thr180 and Tyr182 to promote ICAM-1 protein expression [172-175]. Previously, we demonstrated that melphalan-induced ICAM-1 protein expression was reduced with an inhibitor specific to phosphorylated-p38 (SB202190; 10 $\mu \mathrm{M})$ [90]. Based on these data, we hypothesized that KZ-41 reduction in IR-induced ICAM-1 expression was attributed to modulation of p38-dependent inflammatory signaling. We first performed a time-course experiment to both confirm and identify maximum p38 phosphorylation (Thr180/Tyr182) post-IR (Figure 4-6A). Relative to total p38 levels, phosphorylated-p38 reached a transient plateau over four to eight hours post-IR $(* \mathrm{P}<0.05)$. We then collected and analyzed treated cells four hours following irradiation. In comparison, irradiated RECs that were treated with KZ-41 prior to exposure had 30\% reduction in phosphorylated-p38 (Figure 4-6B, *,**P $<0.05$ ).

\section{Radiation activates p53 in RECs}

Radiation-induced phosphorylation of p53 at serine residues 15, 33, and 37 stabilizes p53 by preventing MDM2-driven polyubiquitination and degradation and has been shown to require the kinase activity of p38 [143, 146]. In RECs, radiation dramatically increased p53 phosphorylation at serine residues 15,33 , and 37 , which in turn, led to accumulation of total p53 (Figure 4-7). The net result was an increased ratio of phosphorylated to total p53 for each of the serine residues examined. Next, we hypothesized that KZ-41, by virtue of inhibiting $\mathrm{p} 38$, reduces radiation-induced serine phosphorylation and accumulation of p53. As hypothesized, pre-incubation with KZ-41 $(10 \mu \mathrm{M})$ diminished p53 phosphorylation and accumulation $(\# \mathrm{P}<0.05)$. 


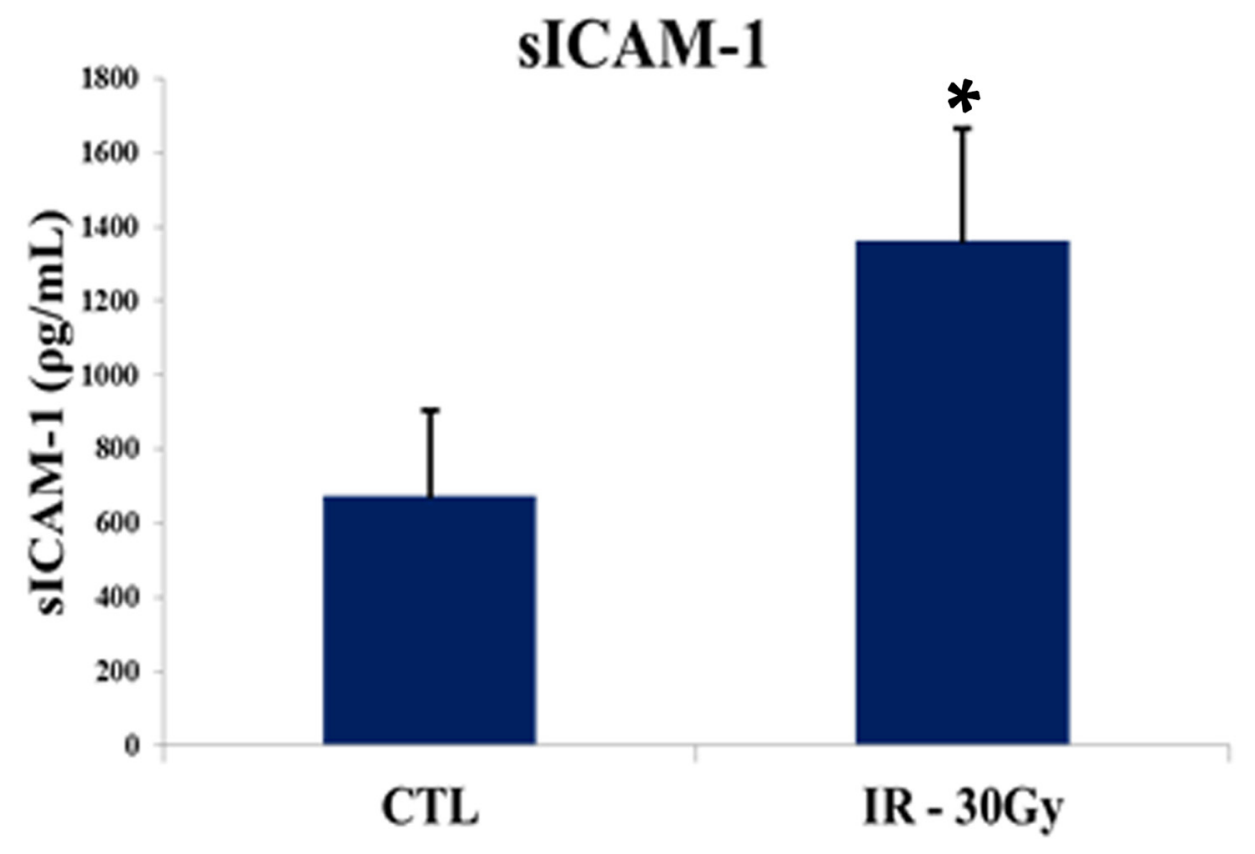

Figure 4-4. Soluble ICAM-1 elevated by high-dose irradiation in RECs.

Soluble-ICAM-1 in REC media was measured by ELISA 24 hours post-IR $\left({ }^{137} \mathrm{Cs} ; 30\right.$ Gy). Results show significant elevation of sICAM-1 levels $(* P<0.05)$. Data represented as mean concentration $\pm \mathrm{SD}$. 


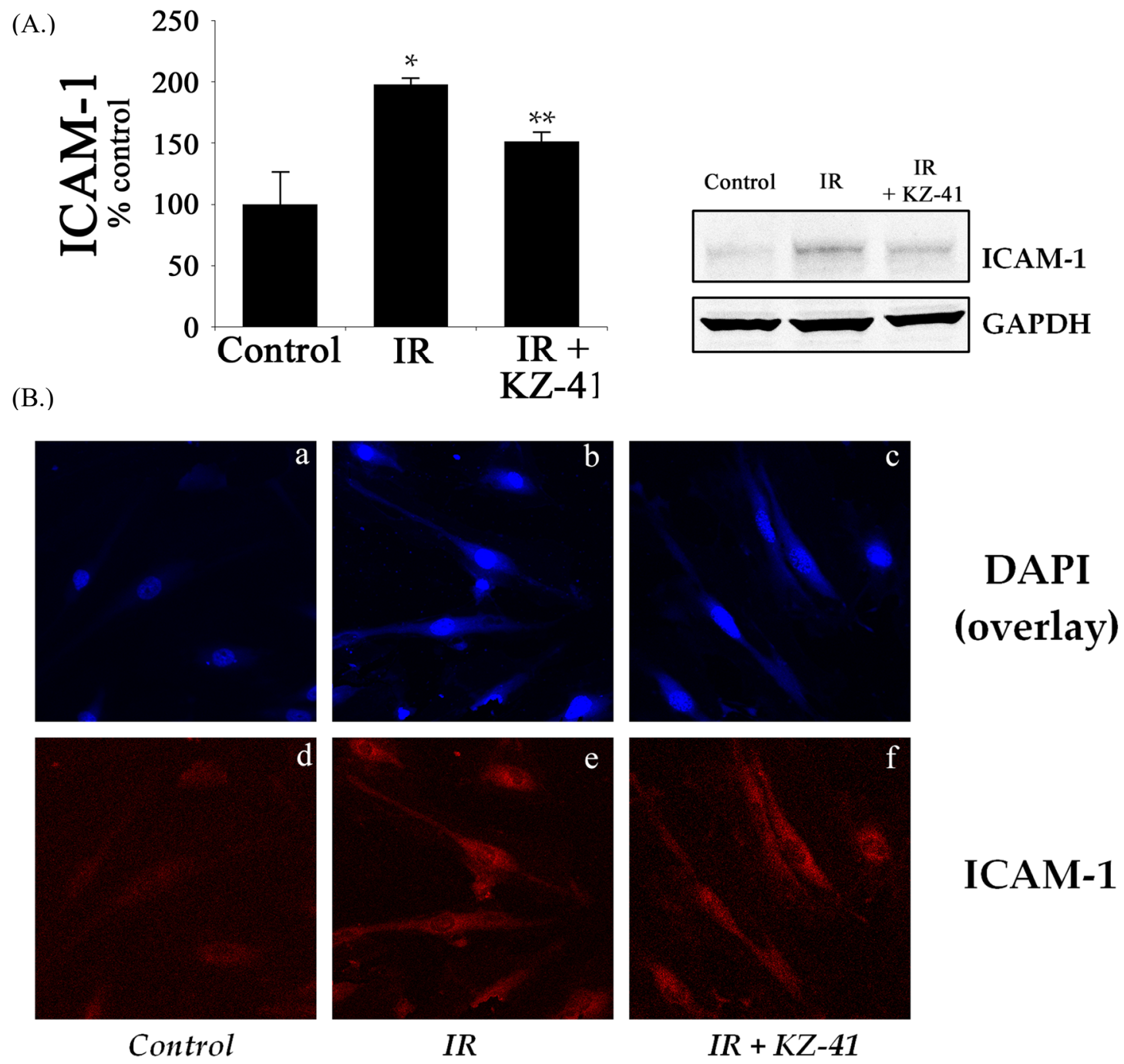

Figure 4-5. KZ-41 decreased levels of ICAM-1 following radiation in RECs.

A) Immunoblotting of ICAM-1 from IR-RECs after 24 hours show a significant upregulation compared to unirradiated cells; treatment with KZ-41 $(10 \mu \mathrm{M})$ reduces ICAM1 levels by nearly $24 \%$ (Mean $\pm \mathrm{SD} ; * \mathrm{P}<0.05, * * \mathrm{P}<0.05 ; \mathrm{n}=3$ ). Data analysis on left, immunoblot images on the right. B) Confocal microscopy of RECs from flow-chamber slides. Top panels (A-C; Control, IR and IR+KZ-41, respectively) represent overlay images of DAPI and ICAM-1. Lower panels, D-E represents ICAM-1 immunoreactivity under confocal microscopy. 

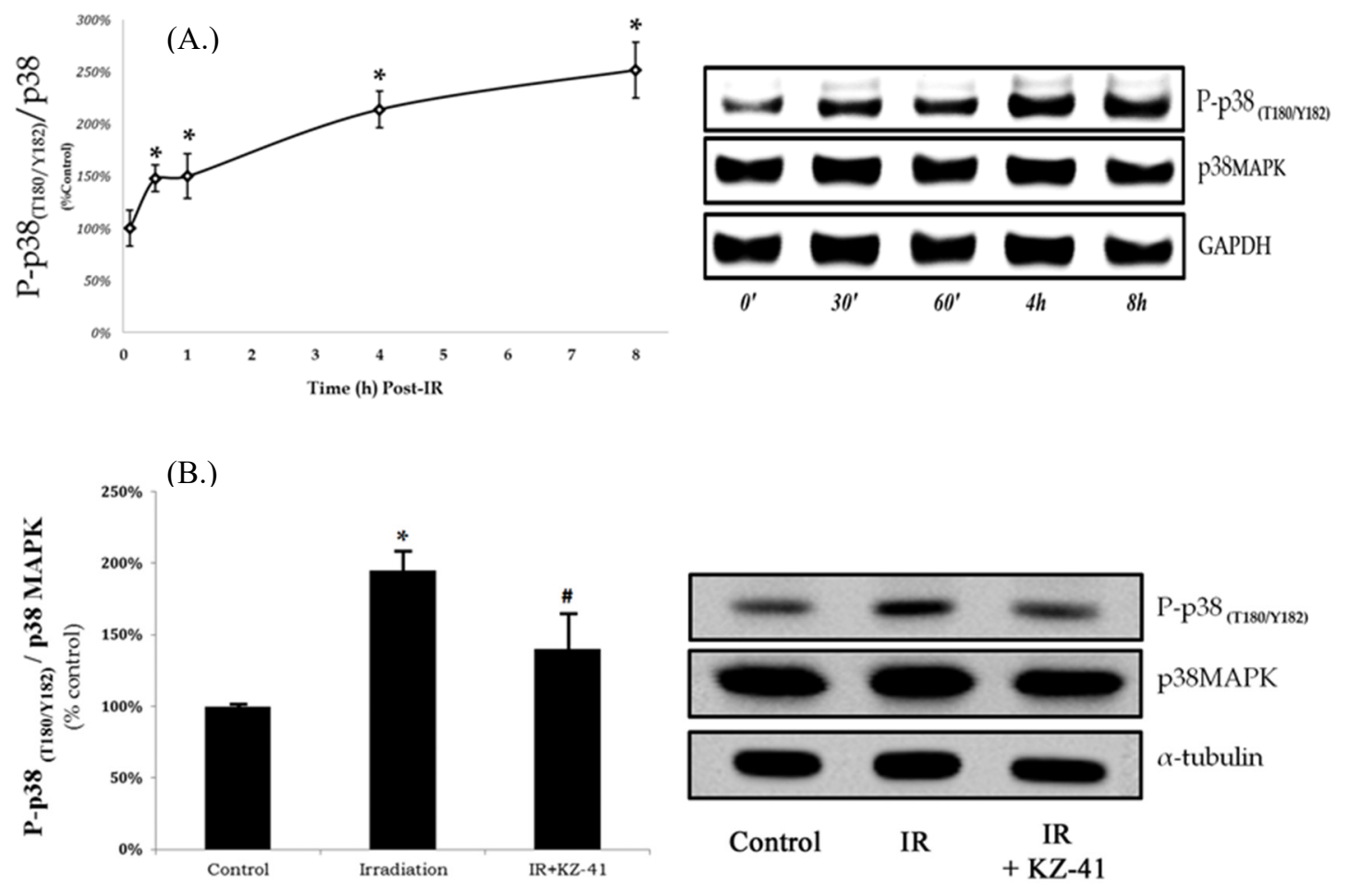

Figure 4-6. KZ-41 inhibits IR-induced p38 phosphorylation in RECs.

A) RECs receiving 30 Gy irradiation in a single fraction show increases in phosphorylation of $\mathrm{p} 38$ (T180/Y182) that reach a plateau between 4-8 hours compared to control cells at the same time-points $(* \mathrm{P}<0.05)$. B) Irradiated RECs with or without treatment of KZ-41 $(10 \mu \mathrm{M})$ were harvested and analyzed for phospho-p38 at 4 hours. KZ-41 treated RECs showed significant reductions in total levels of phosphorylated p38 (T180/Y182), as compared to IR-RECs (\# P $<0.05)$. 

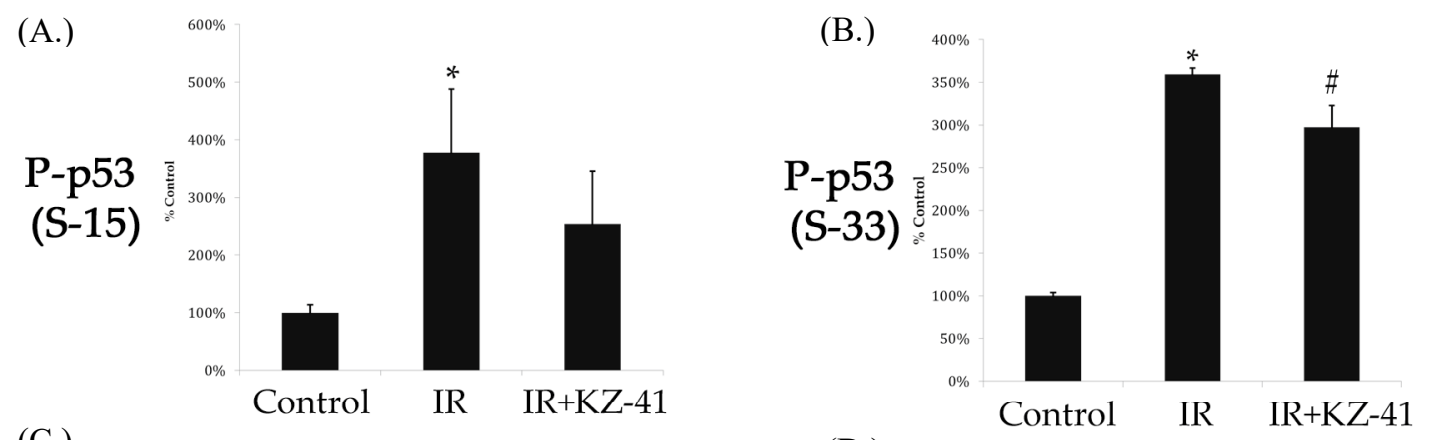

(C.)

(D.)
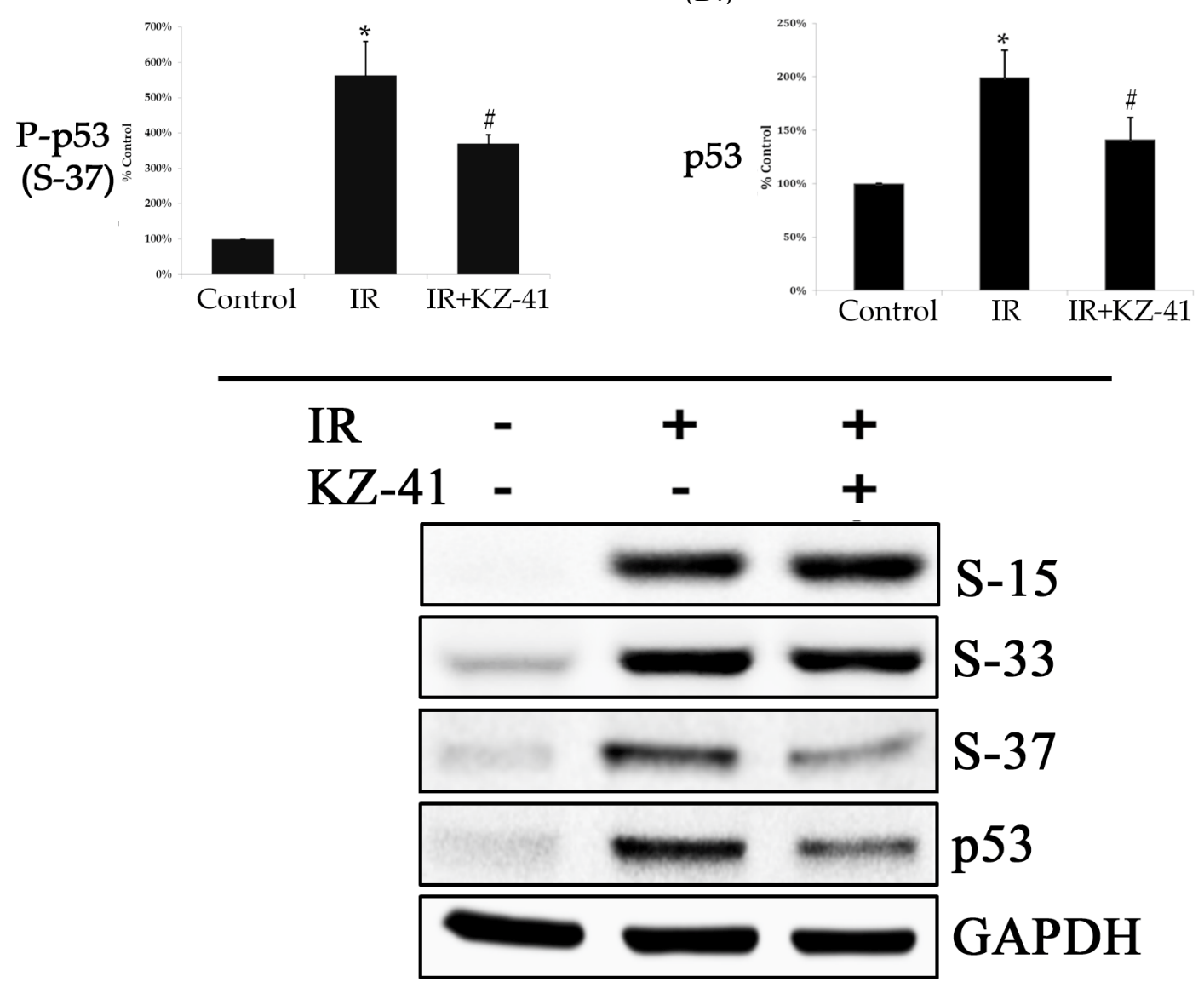

Figure 4-7. KZ-41 reduced p53 protein levels in irradiated RECs.

Phosphorylation at serine 15 (A), 33 (B), 37 (C) corresponded to IR-induced p53 accumulation $(\mathrm{D})(* \mathrm{P}<0.05)$ after 4 hours. The ratio of $\mathrm{p} 53$ phosphorylation at Ser 33 and 37 (relative to GAPDH) in KZ-41-treated $(10 \mu \mathrm{M})$ RECs showed significant reduction $(\# \mathrm{P}<0.05) . \mathrm{D})$ Total p53 protein accumulation revealed significantly reduced levels in KZ-41 treated RECs $(\# \mathrm{P}<0.05)$. 


\section{Radiation-induced REC death prevented with KZ-41}

Apoptotic cell death mechanisms are triggered in certain cell populations in vivo following exposure to high-dose radiation [176]. Activated (cleaved) caspase-3, a crucial effector of the terminal or execution phase of the apoptotic pathway, has been recognized as a reliable phenotypic marker of apoptosis [177]. To examine whether the reduction in p38-mediated p53 induction caused apoptotic signal activation in RECs, we measured the cleavage of caspase-3 using two different assays (ELISA and ICW) following high-dose IR and found that a minority of irradiated RECs showed signs of apoptosis over 24 hours (Figure 4-8; $* \mathrm{P}<0.005$, ELISA; $* \mathrm{P}<0.05 \mathrm{ICW}$ ). Treatment with KZ-41, however, was able to prevent the activation of apoptotic signaling compared to IR controls in both assays $(* * \mathrm{P}<0.05)$.

\section{Radiation induces a paxillin-dependent proliferative phenotype}

Irradiation of the retinal vasculature triggers cell death in a small fraction of cells which absorb the brunt of the exposure [178]. Similarly, we found that a minority of irradiated RECs exhibited signs of apoptosis as measured by cleaved caspase-3 (Figure 4-8). Surviving RECs elicited a compensatory migratory/proliferative response, characterized by enhanced VEGF secretion in the soluble media (Figure 4-9) [34, 176]. We then showed that radiation-induced VEGF secretion corresponded to increased proliferation in surviving RECs (Figure 4-10A, IR vs. Control $* \mathrm{P}<0.05$ ). The proliferative capacity was reduced to control levels in KZ-41 treated irradiated RECs (IR $+\mathrm{KZ}-41$ vs. IR, \#P < 0.05). Inflammation-induced angiogenesis has been shown to depend on p38 phosphorylation and paxillin-dependent cytoskeletal changes [179, 180]. In fact, irradiation has been shown to specifically induce both the expression and phosphorylation of paxillin in A549 cells [181]. Radiation-induced REC proliferation directly correlated with a significant induction in tyrosine phosphorylation of paxillin (Y118) 24 hours following IR (Figure 4-10B, $* \mathrm{P}<0.05$ ). Irradiated RECs treated with either KZ-41 or the p38 inhibitor SB202190 significantly reduced radiation-induced phosphorylation of paxillin $(\# \mathrm{P}<0.05)$. These data suggest that radiation-induced $\mathrm{p} 38$ activation is a mechanisms driving downstream kinase-dependent activation of focal adhesion protein paxillin, resulting in inflammatory-mediated proliferation of RECs.

\section{KZ-41-loaded nanoemulsion delivered effective concentrations to mouse eyes}

We first validated the size and subsequent stability of our ocular nanoemulsion used during in vivo pharmacological testing. Our results indicated that the nanoemulsion (NE) was stable (remained translucent) over a period of 60 days and that the particle size remained below the $100 \mathrm{~nm}$ criteria for nanoemulsion categorization (Figure 4-11). NE physicochemical properties included a viscosity of $17 \mathrm{mPa} \cdot \mathrm{s}$, average particle size of 63.5 $\pm 7.5 \mathrm{~nm}$, and $\mathrm{pH}$ of 6.5 . After 60 days at room temperature, the particle size had increased to $75 \mathrm{~nm}$, but this change was not significant. Table 4-1 details the NE characteristics and particle sizes on $0,7,30$ and 60 days. 
(A.)

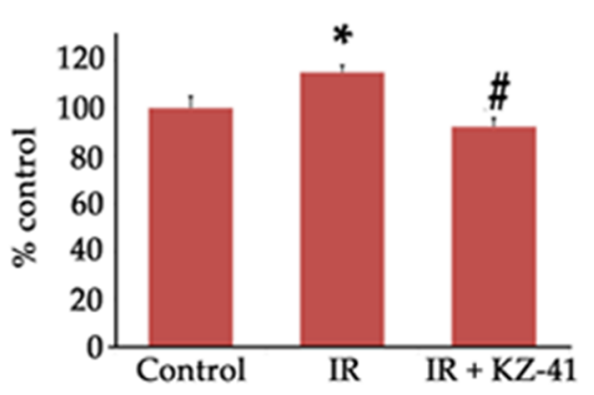

(B.)

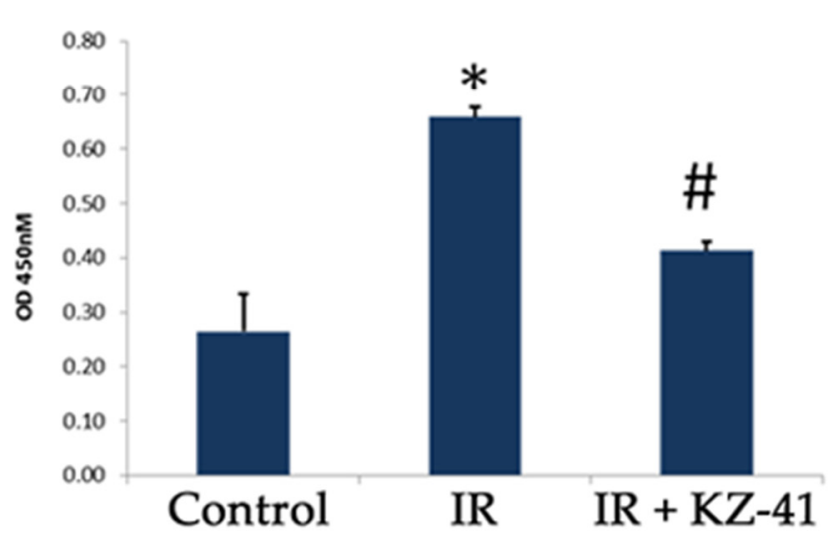

Figure 4-8. KZ-41 reduces IR-induced cleavage of apoptotic marker caspase-3.

Two in vitro assays showed the activation or cleavage of caspase-3 (Asp175) was induced by irradiation ( $* \mathrm{P}<0.05)$. A) In-Cell Western and B) Pathscan ELISA assays confirm that KZ-41 reduces apoptotic signaling in RECs exposed to high-doses of radiation $(\# \mathrm{P}<0.05)$. 


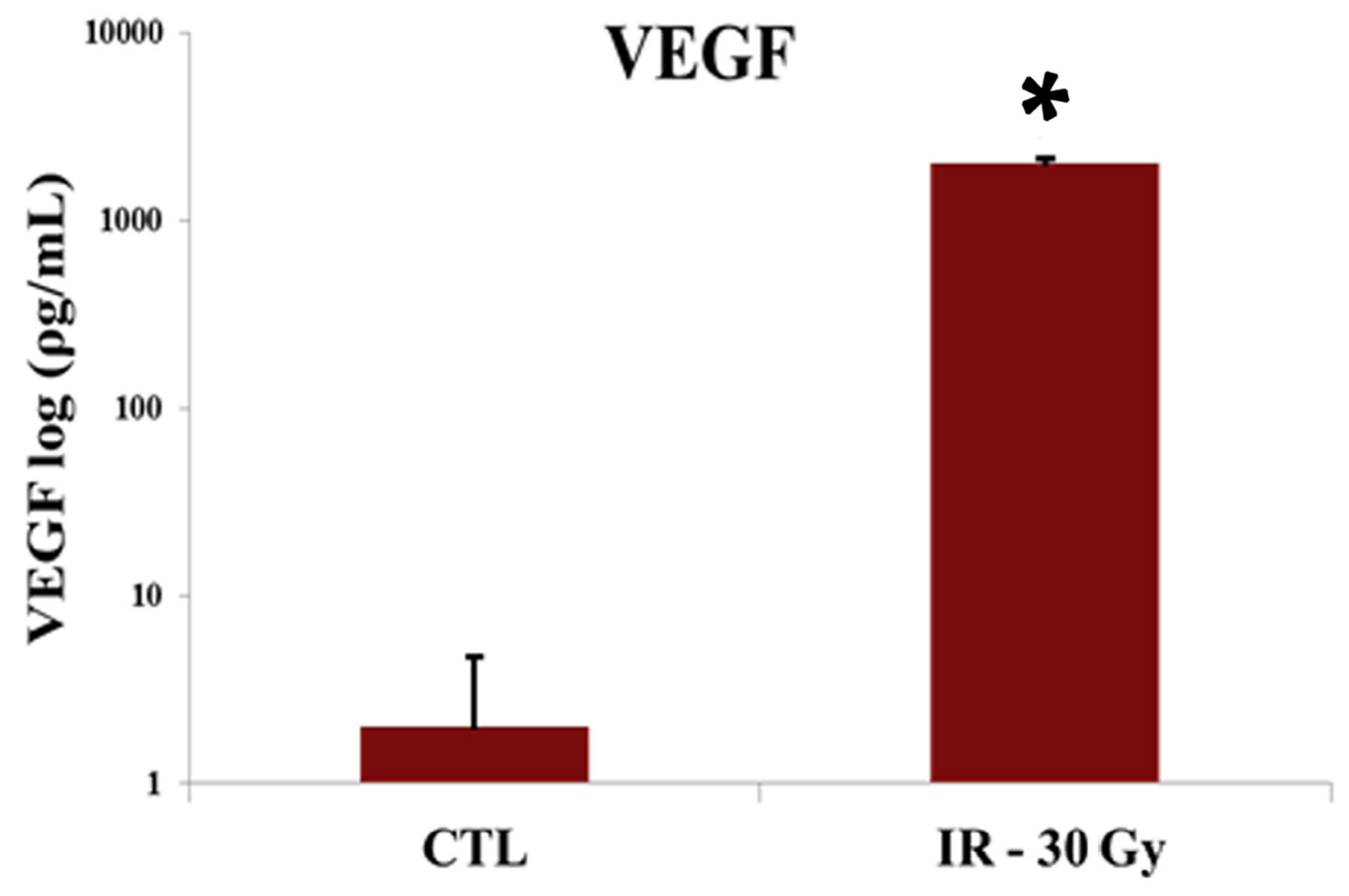

Figure 4-9. VEGF secretion in REC media post-IR as measured by ELISA.

Irradiated REC media was collected after 24 hours following high-dose radiation. Media were analyzed for VEGF concentrations using an ELISA, normalized to total protein found in media. Results showed that with exposures of radiation, RECs secrete the growth factor in high abundance $(* \mathrm{P}<0.001 ; \mathrm{n}=3)$ 


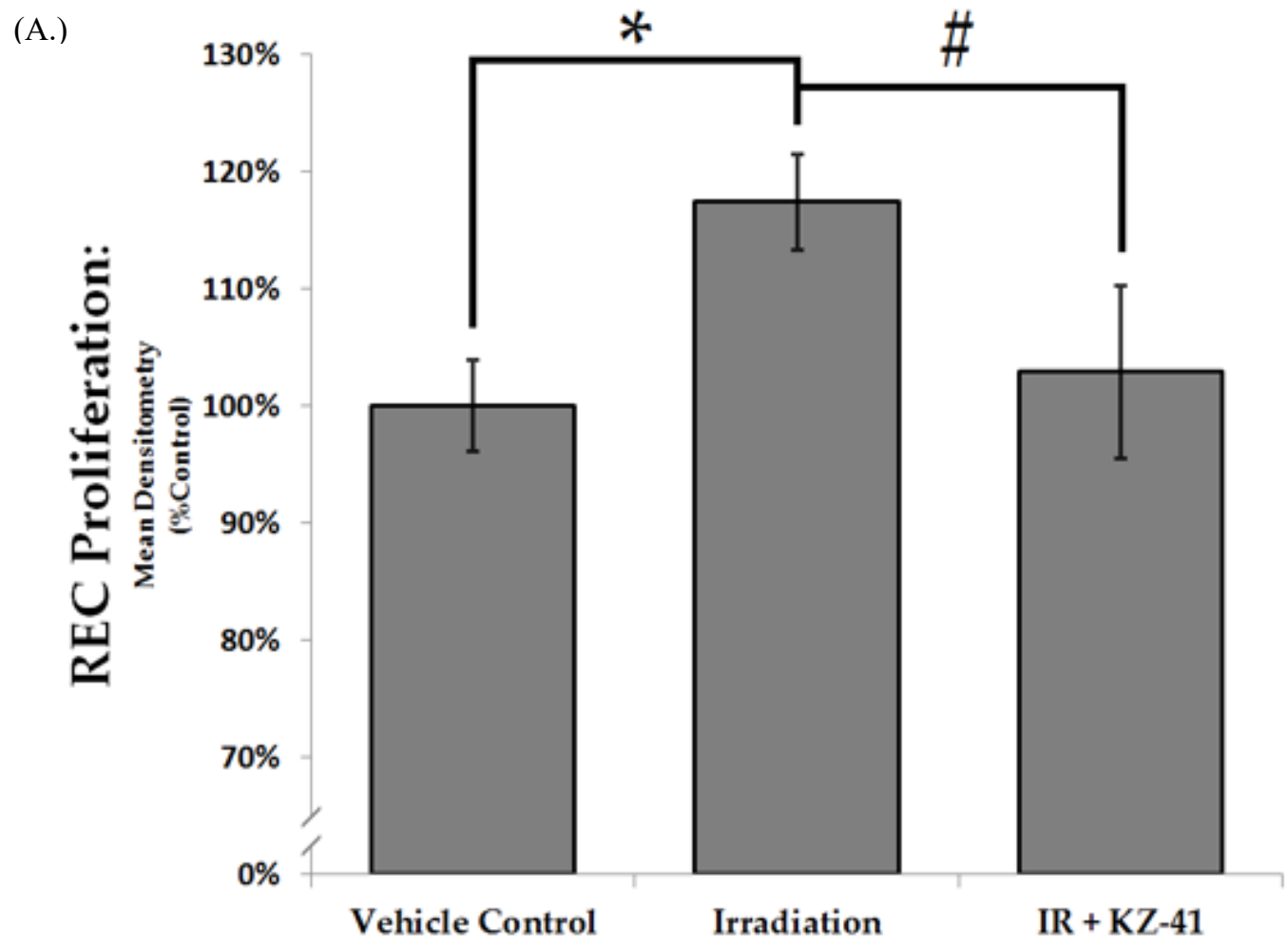

(B.)

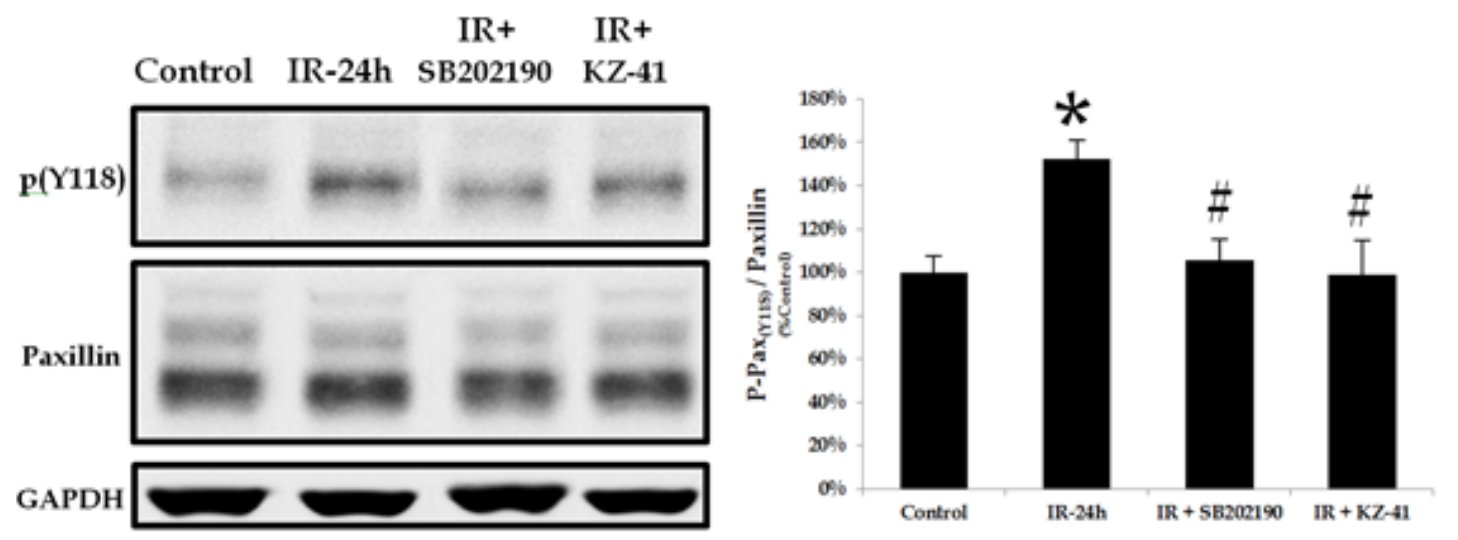

Figure 4-10. KZ-41 prevents IR-induced proliferative activity via paxillin.

A) Irradiation-induced REC proliferation was measured after 24 hours using the WST-1 proliferation assay. REC proliferation was enhanced by irradiation $(* \mathrm{P}<0.05)$ and was significantly reduced with treatment of KZ-41 $(10 \mu \mathrm{M})(\# \mathrm{P}<0.05)$. B) Paxillin phosphorylation (Y118) was measured 24 hours after irradiation using immunoblotting and showed enhanced levels ( $* \mathrm{P}<0.01)$. Both KZ-41 $(10 \mu \mathrm{M})$ and p38 inhibitor SB202190 $(10 \mu \mathrm{M})$ significantly reduced levels of paxillin phosphorylation $(\# \mathrm{P}<0.05)$. 
(A.)

(B.)
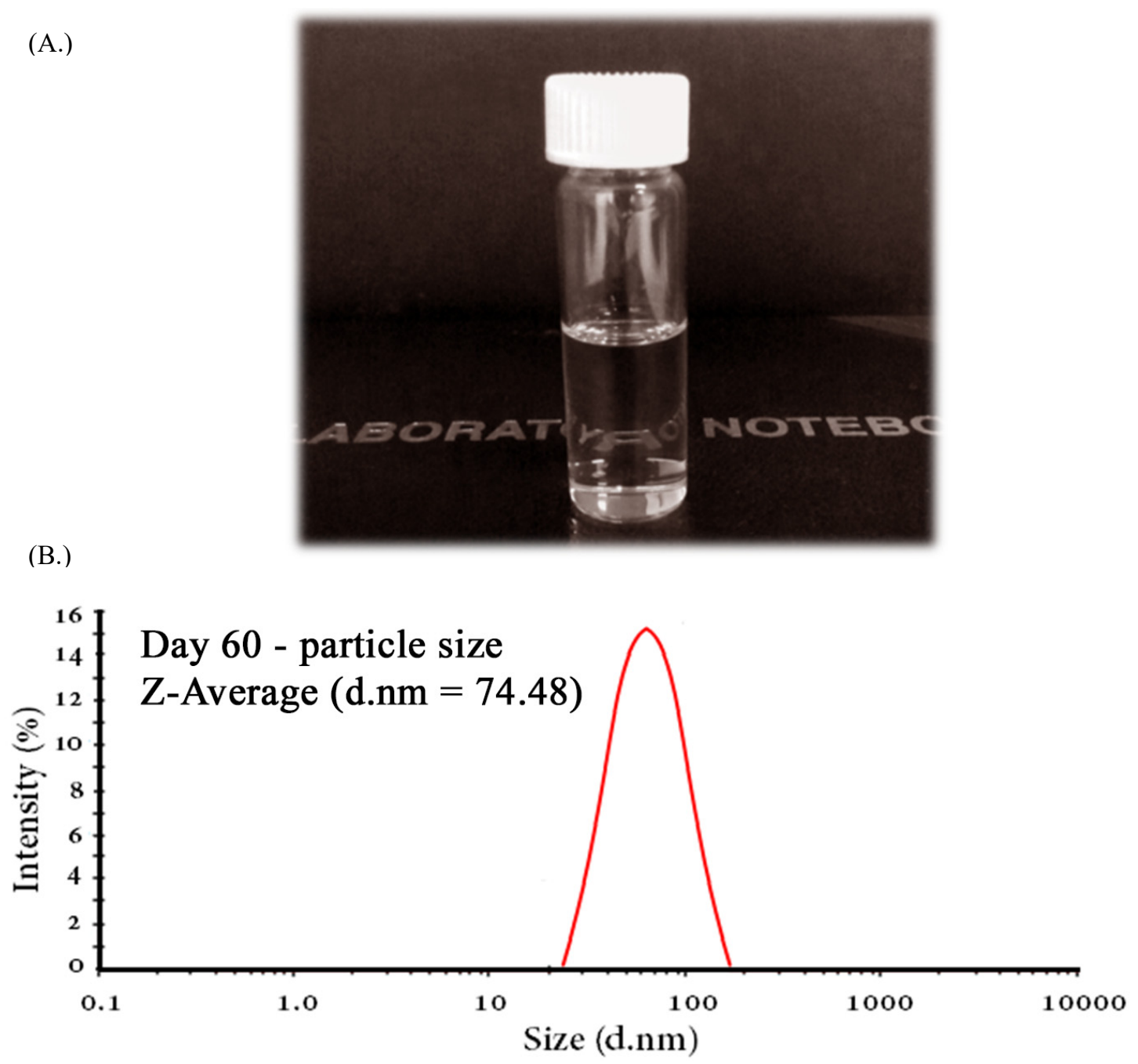

Figure 4-11. Characterization of ocular nanoemulsion.

A) Ocular nanoemulsion remained translucent for 60 days. B) Particle size analysis show at day 60, ocular NE diameter (d.nm) remains below $100 \mathrm{~nm}$. 
Table 4-1. Ophthalmic nanoemulsion characterization.

\begin{tabular}{lcl}
\hline Parameter & Value & SD \\
\hline Viscosity $(\mathrm{mPa} \cdot \mathrm{s})$ & 17.0 & $\pm 1.8 \mathrm{e}-5$ \\
$\mathrm{pH}$ & 6.5 & \pm 0.04 \\
Particle size Day 0 $(\mathrm{nm})$ & 61.3 & \pm 2.0 \\
Particle size Day 7 $(\mathrm{nm})$ & 59.3 & \pm 11 \\
Particle size Day 30 $(\mathrm{nm})$ & 58.7 & \pm 0.3 \\
Particle size Day 60 $(\mathrm{nm})$ & 74.5 & \pm 4.0 \\
\hline
\end{tabular}

Notes: nm: nanometer; SD: standard deviation 
Method validation chromatograms of blank plasma and eye homogenates, as well as KZ-41 in plasma and eye homogenates after ocular administration are depicted in Figure 4-12. Ocular pharmacokinetic analysis has confirmed penetration of KZ-41 through the corneal tissue within 5 minutes (Figure 4-13). Nanoemulsion delivery produced a mean peak eye homogenate concentration of $130.1 \pm 4.6 \mathrm{mg} / \mathrm{g}$ at 15 minutes. KZ-41 concentration in the eye dropped exponentially, with a half-life of $2.1 \pm 0.4 \mathrm{~h}$. The volume of distribution was estimated at $1.7 \pm 0.4 \mathrm{~L} / \mathrm{kg}$, and ocular clearance was $0.57 \pm$ $0.07 \mathrm{~mL} / \mathrm{h} / \mathrm{g}$. Systemic circulation was achieved 30 minutes after ocular administration, correlating precisely with the apparent distribution of KZ-41 out of the eye, though exposures, as measured by AUC, were significantly less than that of eye homogenates. Table 4-2 lists these parameters.

\section{KZ-41 reduces avascular area and neovascularization in the murine OIR model}

The most frequently used in vivo model for studying the effect of genomic or pharmacologic manipulation of key signaling proteins on the natural history of proliferative retinopathies (e.g., RR) is the murine oxygen-induced retinopathy (OIR) model $[161,162,164]$. In the murine OIR model, retinal expression of phosphorylated p38 is enhanced [182]. We used the OIR model to test the hypothesis that KZ-41 would prevent RNV driven by oxidative stress and ischemic injury. Mouse pups received daily ocular administration of either KZ-41 (100 mg/kg; treated eye) or vehicle (ophthalmic NE; contralateral eye) from P12 to P17. As shown in Figure 4-14 (panels A-D; Normoxia-N17, OIR17-untreated, OIR17+Vehicle, and OIR17+KZ-41), hyperoxia led to significant vaso-obliteration of the central retina of mouse pups (P7-P12). Both untreated and vehicle-treated retinas showed significantly larger avascular areas surrounding the optic disc as compared to normoxia controls $(20.5 \pm 1.8,18.6 \pm 3.1$ vs. $4.4 \pm 1.1 \mathrm{AV} \%$ area, $\left.{ }^{*} \mathrm{P}<0.001\right)$.

Significant neovascularization was also noted in both untreated and vehicletreated retinas when compared to normoxia controls (Figure 4-15, panels A-C, $24.7 \pm$ $2.3,22.3 \pm 1.4$ vs. $0.76 \pm 0.28 \mathrm{NV} \%$ area, $\left.{ }^{*} \mathrm{P}<0.005\right)$. Neither total avascularity nor neovascularization differed between untreated and vehicle-treated retinas $(\mathrm{P}>0.05)$. KZ41 significantly reduced avascularity (8.6 vs. $18.6 \mathrm{AV} \%$ area, $\# \mathrm{P}<0.001)$ and neovascularization (16.5 vs. $22.3 \mathrm{NV} \%$ area, $\# \mathrm{P}<0.01$ ) compared to vehicle-control retinas. These results suggest that KZ-41 reduces the extent of pathological RNV while not affecting normal revascularization under OIR conditions.

\section{Discussion}

Severe microvascular injury has been touted as the primary mechanism in the pathogenesis of radiation-induced tissue damage [14]. By focusing on radiation injury to the retinal vasculature, we were able to induce inflammatory signaling that parallels pathways seen in DR and neovascular AMD. These retinal diseases manifest in similar ways and all comprise a neovascular tendency leading to visual impairments. 

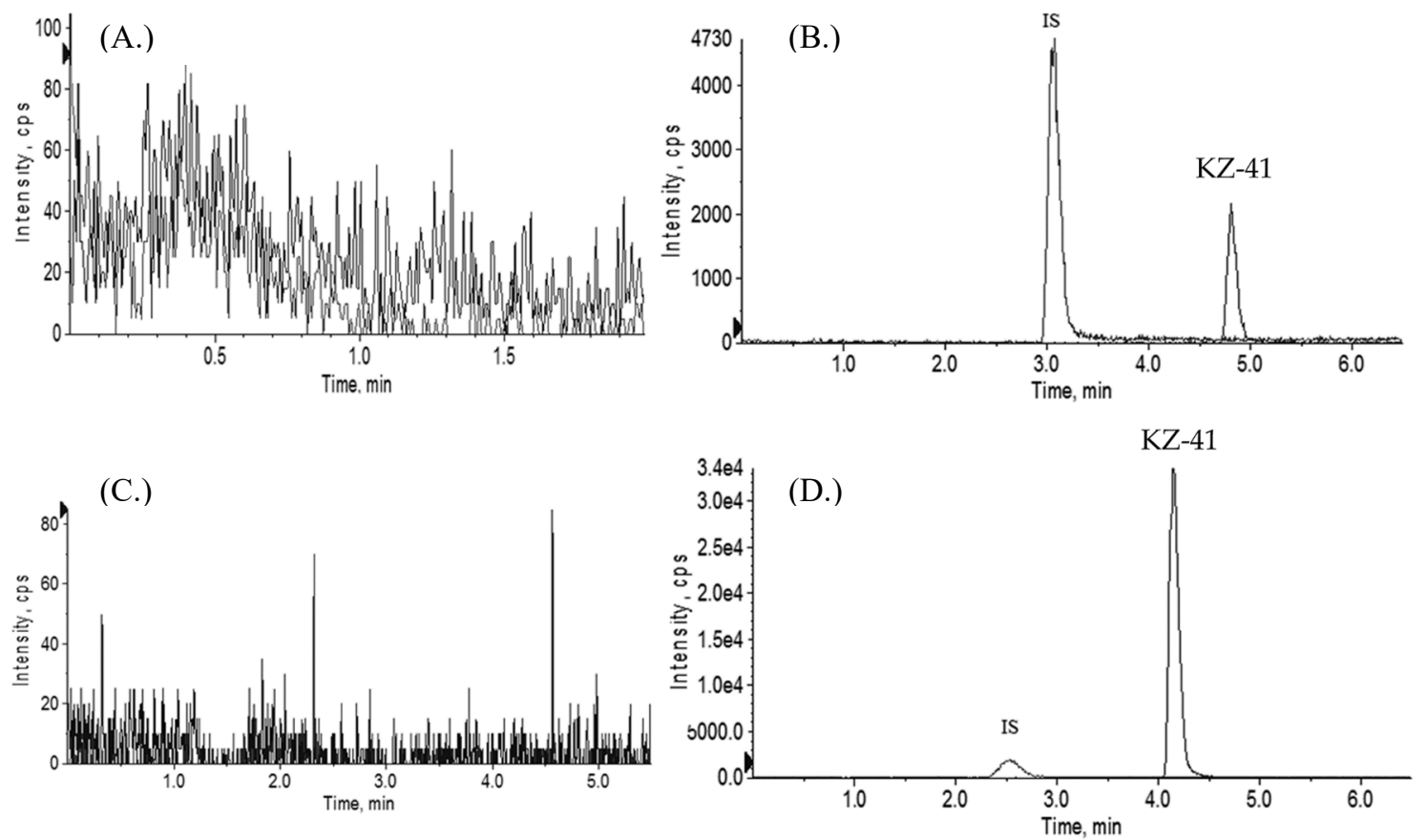

Figure 4-12. LC-MS/MS chromatograms of KZ-41.

(A) Blank plasma; (B) mice plasma after ocular administration; (C) Blank eye homogenate; (D) eye homogenates after ocular administration show sensitivity of method to detect KZ-41. 

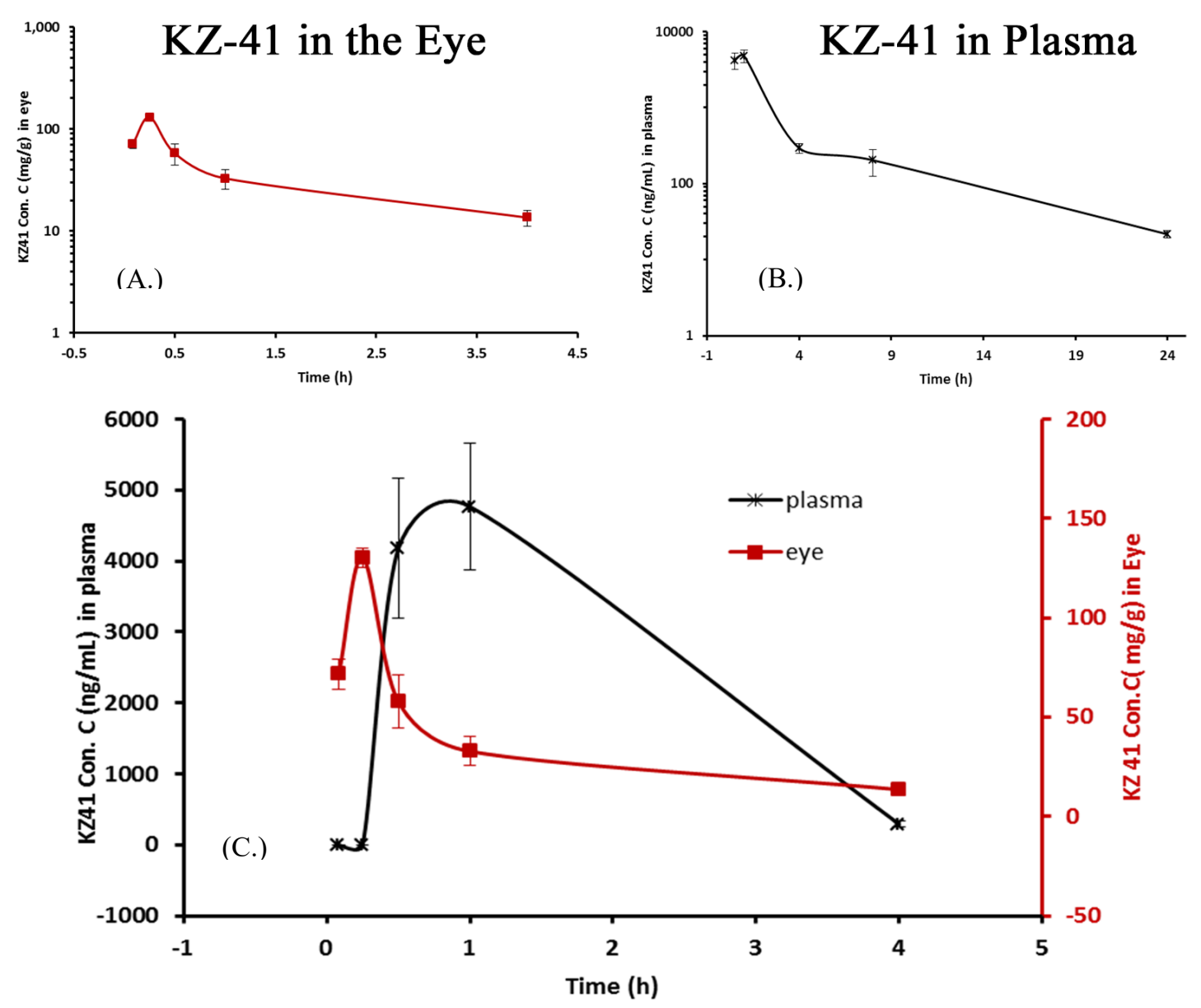

Figure 4-13. Intra-ocular and plasma concentrations of topical KZ-41.

A) Concentration-time profiles of KZ-41 in the eye and B) in the plasma. C) Overlay of concentration-time profiles of KZ-41 in mouse eye and plasma shows penetration into the ocular tissues within 5 minutes of administration and peaked at 15 minutes. Plasma concentrations were detectable 30 minutes following ocular administration. $(n=3$ mice/time point). 
Table 4-2. Eye and plasma concentrations after ocular administration of KZ-41.

\begin{tabular}{lcccc}
\hline \multirow{2}{*}{ Parameter } & \multicolumn{4}{c}{ KZ-41 (100 mg/kg) } \\
\cline { 2 - 5 } & Eye & [units] & Plasma & [units] \\
\hline $\mathrm{C}_{\max }$ & $130.00(4.6)$ & {$[\mathrm{mg} / \mathrm{g}]$} & $4.8(0.78)$ & {$[\mu \mathrm{g} / \mathrm{mL}]$} \\
$\mathrm{t}_{\max }$ & $0.25(0.01)$ & {$[\mathrm{h}]$} & $0.83(0.29)$ & {$[\mathrm{h}]$} \\
$\mathrm{AUC}$ & $0.18(0.02)$ & {$[\mathrm{hr} * \mathrm{mg} / \mathrm{mL}]$} & $0.014(0.003)$ & {$[\mathrm{hr} * \mathrm{mg} / \mathrm{mL}]$} \\
$\mathrm{t}_{1} / 2$ & $2.06(0.42)$ & {$[\mathrm{h}]$} & $5.23(0.14)$ & {$[\mathrm{h}]$} \\
$\mathrm{CL}$ & $0.57(0.07)$ & {$[\mathrm{mL} / \mathrm{hr} / \mathrm{g}]$} & $7.53(1.91)$ & {$[\mathrm{mL} / \mathrm{hr} / \mathrm{g}]$} \\
$\mathrm{Vd}$ & $1.70(0.37)$ & {$[\mathrm{mL} / \mathrm{g}]$} & $56.60(12.9)$ & {$[\mathrm{L} / \mathrm{mg}]$} \\
\hline
\end{tabular}

Note: Values are expressed as mean $\pm(\mathrm{SD}) ; \mathrm{C}_{\max }$ : Maximum concentration after ocular administration and penetration into circulation; AUC: area under the curve; $\mathrm{T}_{1 / 2}$ : half-life; $t_{\max }$ : time to reach maximum concentration; Vd: volume of distribution. 

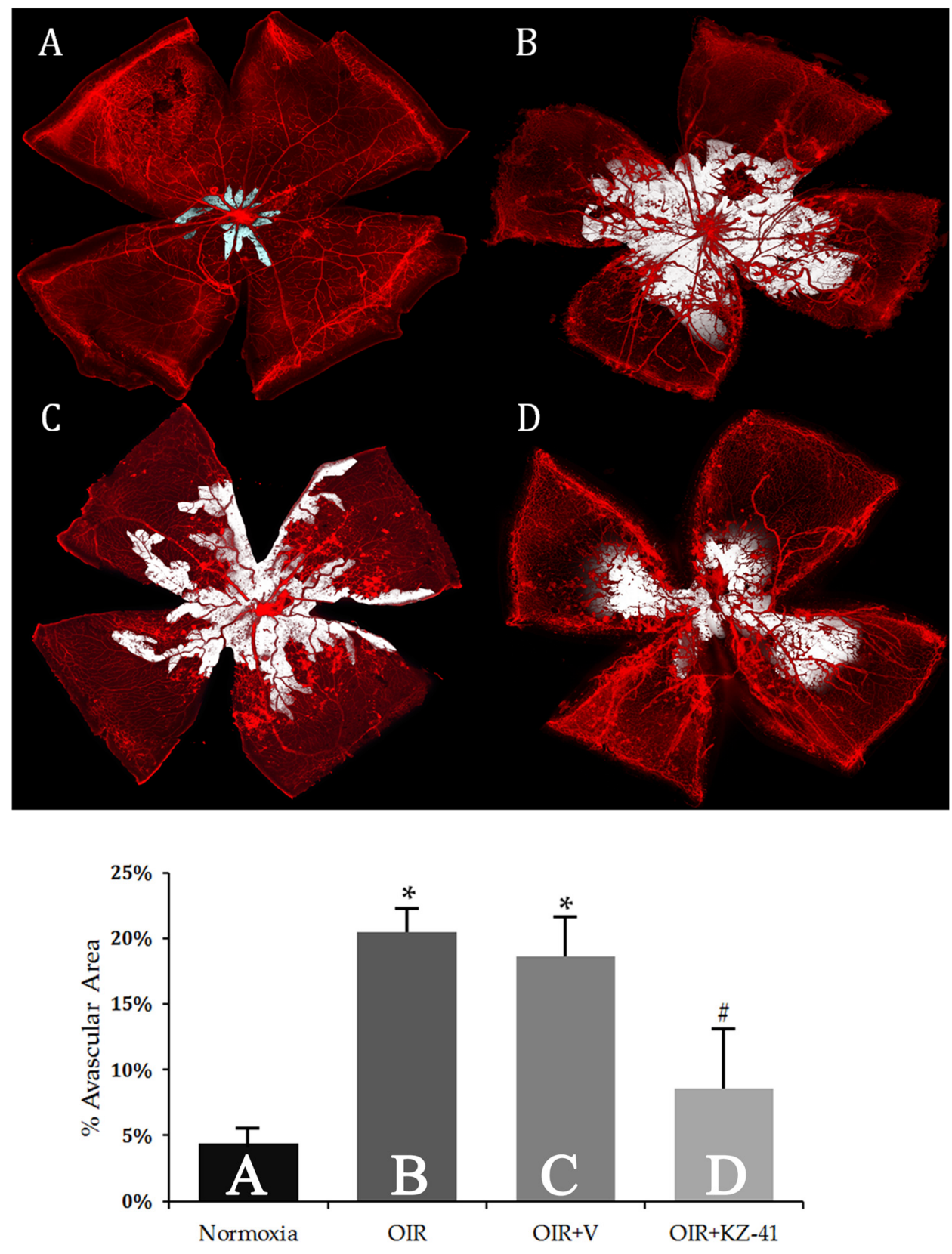

\section{Figure 4-14. KZ-41 reduces avascular area.}

A-D) P17 flat-mounted retinas were stained for endothelial cells using isolectin-B4 (red): Normoxia, OIR, OIR $+\mathrm{V}$, OIR $+\mathrm{KZ}-41$, respectively. Mice received daily ocular administration of either KZ-41 (100 mg/kg) or vehicle (ocular nanoemulsion) from P12 to P17. Avascular area was determined using software-assisted analysis; shown in white. OIR mice show significant avascular area as compared to normoxia controls $(* \mathrm{P}<$ $0.001)$. KZ-41 lowered area percent avascular area by nearly $50 \%$ (\#P $<0.001$ ). Images were acquired at 10x magnification and digitally stitched together to show the entire retinal vasculature. Data represent mean $( \pm S D) . N=5 /$ group. 

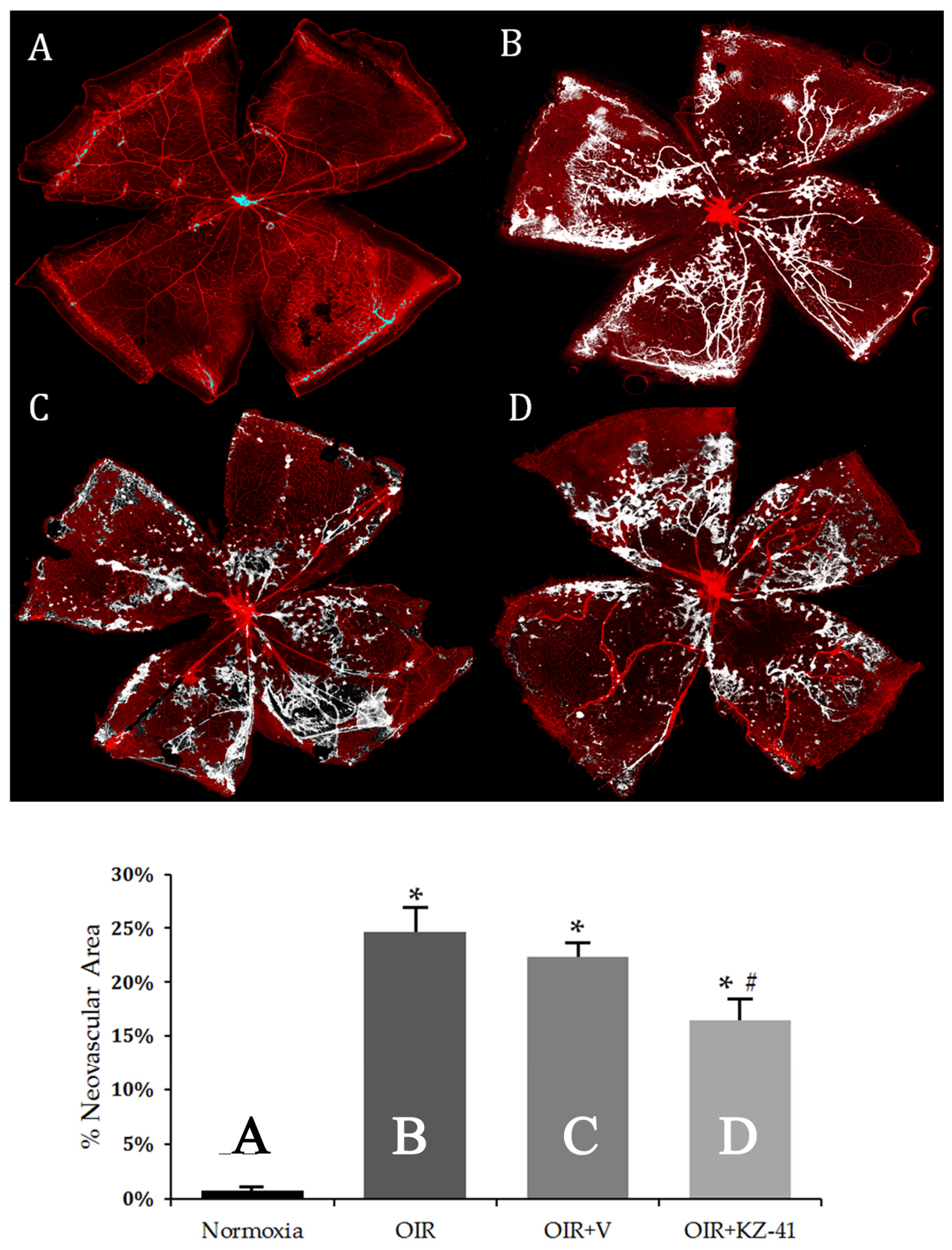

Figure 4-15. KZ-41 reduces neovascularization.

A-D) P17 retinas stained for endothelial cells using isolectin-B4 (red) were analyzed for neovascularization: Normoxia, OIR, OIR+V, OIR+KZ-41, respectively. Analysis was performed after setting threshold limits to disregard non-neovascular networks and larger vessels in and around the optic disc (high intensity tufts shown in white). Both OIR groups (untreated and vehicle-treated) showed extensive tufting compared to normoxia controls ( $* \mathrm{P}<0.005)$. KZ-41 decreased neovascular tufts by $\sim 30 \%$ from OIR + Vehicle $(\# \mathrm{P}<0.01)$. Images were acquired at $10 \mathrm{x}$ magnification and digitally stitched together. Data represent mean $( \pm \mathrm{SD}) . \mathrm{N}=5$ /group. 
Studies have emphasized the primary mechanism of retinal injury involves leukocyte entrapment and accumulation within microvascular circulation with resultant capillary closure and subsequent ischemia [15]. ICAM-1 is one of the most recognizable initiators of leukocyte-endothelial cell adhesion [19] and has been highly correlated with ocular inflammatory disorders such as diabetic retinopathy, retinopathy of prematurity, and RR [20-24]. ICAM-1 not only facilitates the adhesion and transmigration of circulating leukocytes into the site of tissue damage, but also exacerbates inflammation through the same signaling cascade driving its surface expression, p38.

In this chapter, we have demonstrated that absorbed doses of radiation to the human REC provoked an inflammatory response characterized by rapid induction in $\mathrm{p} 38$ stress kinase-mediated pathways and downstream effectors, e.g., tumor suppressor, p53 and ICAM-1. We have previously demonstrated that the quinic acid derivative KZ-41 modulates cellular responses to genotoxic stress via mechanisms involving disruption of p38 signal transduction $[88,90,183]$. We extended these findings by demonstrating that KZ-41 also modulates p38 activity in the irradiated REC. KZ-41 blunts p38 activity following radiation exposure, reducing p53 activation, ICAM-1 expression, and adhesion of leukocytes to the inflamed RECs.

Our results show an ICAM-1 dependent increase in leukocyte adhesion in radiation-induced injury to RECs. Disruption of ICAM-1 signaling, either through gene knockout or antibody blockade, prevents VEGF-mediated pathological angiogenesis $[184,185]$. Irradiation-induced activation of $\mathrm{p} 38$ is not limited to promoting acute inflammatory responses (i.e., leukocyte adhesion), but is also capable of propagating chronic inflammatory phenotypes, such as the migration and proliferation of cells through cytoskeletal protein effectors such as focal adhesion kinases (FAK) and the scaffolding protein, paxillin [181].

Focal adhesions (FAs) containing paxillin•FAK complexes coordinate traction and retraction of cellular protrusions and direct movement of dividing and proliferating cell populations [186]. Disruptions in paxillin-coordinated cellular movement through site-directed mutagenesis of key paxillin phosphorylation sites Y31/Y118 or serine 178 reduce FA turnover kinetics and hinder the proliferative/migratory phenotype of endothelial cells [186, 187]. Additionally, genetic knockdown of focal adhesion proteins prevents nascent focal adhesion formation thereby reducing VEGF-mediated RNV in the murine OIR model [188]. Non-specific p38 inhibition has also been shown to reduce vaso-obliteration and neovascular tuft formation in OIR [189]. Not surprisingly, both p38 and paxillin are key regulators of the VEGF-dependent angiogenic response in endothelial cells $[190,191]$. We have shown that in RECs, radiation injury triggers proliferative cell motility through $\mathrm{p} 38$-dependent activation of paxillin and the treatment of KZ-41 prevents this proliferative phenotype by reducing p38-dependent paxillin phosphorylation. Uncoupling p38 and paxillin signal transduction thus offers a potential strategy to design novel therapeutics for the treatment of pathologic retinal neovascularization. 
The aforementioned data, suggest a potential causal link between p38 and proliferative retinopathy following acute radiation injury. We hypothesized that KZ-41 would halt progression of pathologic neovascularization in the murine OIR model. We tested this hypothesis by first developing an ocular nanoemulsion drug delivery system to facilitate non-invasive multiple drug dosing in the neonatal mouse. First, we showed that our nanoemulsion was stable over 60 days and that during this time we still achieved a particle size of $<100 \mathrm{~nm}$. These results suggest that the formulation we used is effective in delivering KZ-41 via the topical route. The ocular nanoemulsion by itself (i.e., without KZ-41) appears not to affect primary study endpoints since both untreated and vehicletreated eyes of OIR mice demonstrated expansive avascular areas around the optic disc as well as extensive neovascular tufting. In contrast, daily ocular administration of KZ-41loaded nanoemulsion during this period of hypoxia decreased ischemic retinopathy and significantly reduced neovascular tufting. Moreover, the effect of KZ-41 appears to be local, as opposed to systemic, since the KZ-41-treated eyes had significantly less pathologic RNV features compared to the contralateral eye, which received vehicle alone.

To correlate in vivo findings with KZ-41 penetration after topical administration, we performed a preliminary ocular pharmacokinetic study in adult age mice. Due to the inherently limited vitreous volume in mouse eyes, we showed that KZ-41 penetrated into the ocular tissue, as shown by detectible drug levels in eye homogenates. Thus, this preliminary PK study confirmed that drug penetration was possible using our NE delivery vehicle which validated our significant findings from OIR mice treated once daily with KZ-41-loaded NE. Further work to decipher the compartments reached and concentrations achieved in the posterior retina will be necessary to optimize dosing regimen needed for maximum effect.

In conclusion, the human REC radiation injury response includes activation of p38-dependent signal transduction pathways that culminated in a pro-angiogenic phenotype. Coordinated cytoskeletal rearrangements required for migration and proliferation are supported by enhanced expression, activation, and recruitment of cell adhesion proteins to focal adhesions. Further, our data suggest that KZ-41, through specific modulation of $\mathrm{p} 38$ and its downstream effectors (i.e., p53, ICAM-1, and paxillin), effectively reduces both the inflammation associated with radiation and the pathological manifestations of p38-dependent RNV (Figure 4-16). Additional insight into the mechanism(s) of action, including identification of the cellular target, are required to fully explore the therapeutic potential of quinic acid derivatives such as KZ41 in treating retinal inflammatory disorders. 


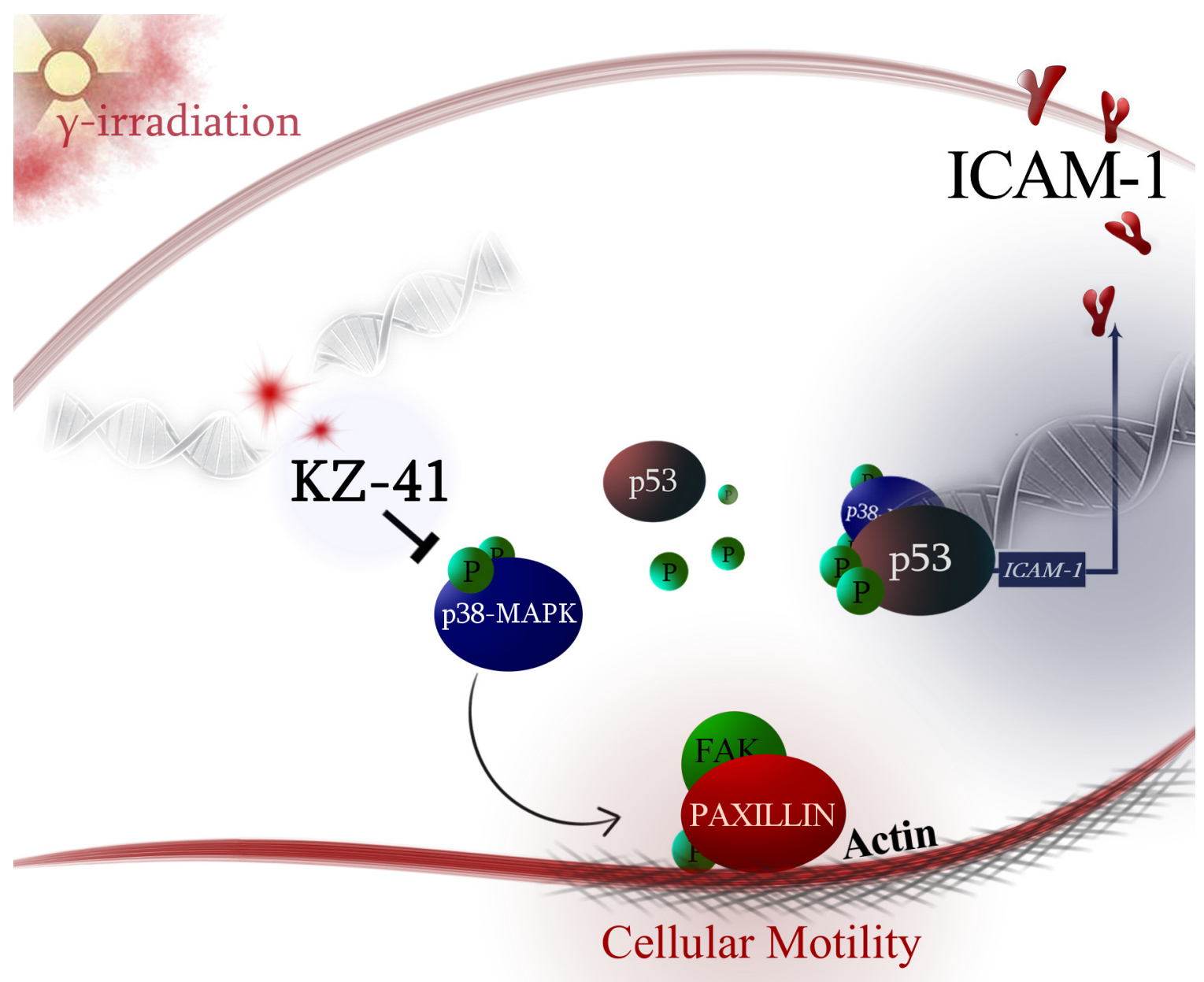

Figure 4-16. Summary model of KZ-41 radioprotective mechanism-of-action.

Gamma- $(\gamma)$ radiation-induced DNA double strand breaks (DSBs) trigger phosphorylation of p38 which results in p53 accumulation and enhances ICAM-1 surface levels. These activities incite a proliferative/migratory phenotype through paxillin phosphorylation, mediated by p38 activation. KZ-41 reduces phospho-p38 and effectively uncouples p38 MAPK signaling to reduce REC inflammation and halt aberrant cellular motility. Therefore, KZ-41 is able to protect RECs against acute radiation injury and the resultant dysfunction of the retinal vasculature. 


\section{CHAPTER 5. DEVELOPMENT OF PAXILLIN MODULATOR, JP-153 FOR THE TREATMENT OF NEOVASCULAR EYE DISEASE}

\section{Introduction}

Diabetes, radiation injury, or years of environmental stress can all contribute to chronic inflammation of retinal tissues, leading to ischemia-driven late stage neovascular eye disease (Figure 5-1). Vision loss occurs in the advanced stages of these diseases due to aberrant ocular angiogenesis and neovascularization [192, 193]. Vascular endothelial growth factor, VEGF plays a key role in this pathophysiology and is the target of current FDA approved anti-angiogenic protein therapeutics [194-198]. However, prospective studies show a decline in long-term efficacy, which is believed to be due to the emergence of VEGF-independent mechanisms and expression of other growth factors and cytokines involved in maintaining the abnormal angiogenic milieu $[52,57]$. In addition, the further decline in visual function with long-term anti-VEGF therapy has been linked to the loss of the choroidal blood supply which is in part, VEGF-dependent and which supports the integrity and health of the overlying retinal pigment epithelium (RPE) and neural retina $[61,199,200]$. Thus, targeting downstream signaling proteins linked to pathological neovascularization represents an alternative or adjunctive approach to approved anti-VEGF treatments, which may reduce the damaging effects of antiangiogenic therapy.

We have identified a proliferative response phenotype of primary human retinal endothelial cells (REC) exposed to high-dose ionizing radiation (Chapter 4) [109]. Irradiation enhanced paxillin Y118 phosphorylation, which was reduced by MAPK inhibition. Under these same mechanisms, inhibiting MAPK and thus, paxillin phosphorylation inhibited in vivo retinal angiogenesis. Our data suggested a direct role for activated paxillin in radiation-induced retinopathy, an ischemic inflammatory disease with a neovascular component [201,202]. The mechanisms by which paxillin coordinates angiogenic signaling through the FAC is not well understood, as most focus has been on targeting kinase activity of either Src or FAK. It was shown however, that paxillin deletion caused dysfunctional cell spreading and stunted migration, similar to the phenotypes of cells without FAK [76-78].

Thus, it was our hypothesis that the activation of downstream effector proteins of the focal adhesion signaling complex (FAC) could represent a viable targeting strategy to undermine the expanse of new blood vessels in the retina. Together with the identification of integrin/adhesion-related genes in our BXD study (Aim 1, Chapter 3) and the radio-mitigating/anti-inflammatory effects of KZ-41 (Aim 2, Chapter 4) we hypothesized that by targeting paxillin-dependent FA signaling, we could effectively prevent neovascular eye disease, regardless of the primary insult and irrespective of specific growth factor secretion. In this chapter, we exploited paxillin as our molecular target and have discovered a novel class of small molecule modulators of the FA protein interaction essential for neovascularization. 


\section{VEGF}

VEGFR-2
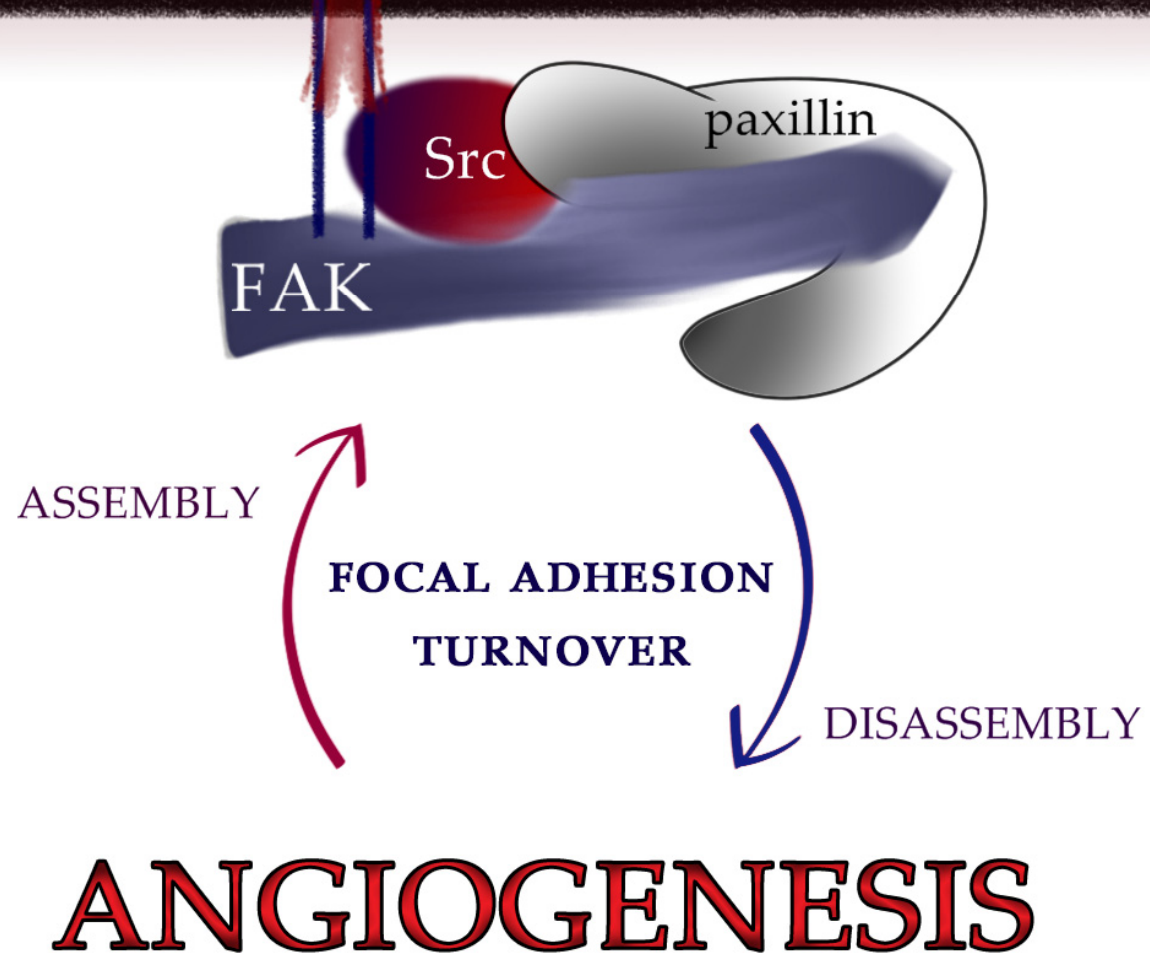

Figure 5-1. VEGF-induced focal adhesion activation of endothelial cells.

Activation of VEGFR-2 by VEGF triggers the formation of the focal adhesion complex (FAC) consisting of Src, FAK and paxillin. Signal transduction through this complex promotes angiogenesis and may represent a novel strategy for the treatment of neovascular eye disease. 


\section{Materials and Methods}

\section{Reagents and antibodies}

Recombinant human VEGF-165A protein was purchased from R\&D Systems (Minneapolis, MN). Total VEGFR-2, Akt, p44/42 MAPK (ERK1/2), cleaved and total PARP, GAPDH, phosphorylated VEGFR-2 (Tyr1175), FAK (Y397, Y576/577, Y925), Akt (Ser473), and ERK 1/2 (Thr202/Tyr204) were all acquired from Cell Signaling (Danvers, MA). Paxillin (Y118) and FAK (Y861) were purchased from Abcam (Cambridge, MA). Mouse antibodies against human paxillin (clone 349) and FAK (clone 77) were purchased from BD Biosciences (San Jose, CA). Mouse alpha( $\alpha$ )-tubulin primary antibody and secondary antibodies, IRDye 800CW goat anti-rabbit, and IRDye 680LT goat anti-mouse were purchased from LI-COR. Calcein-AM was obtained from BD Biosciences. DAPI stain was purchased from Pierce (Rockford, IL). 6-B345TTQ and the Src kinase inhibitor, SU6656 were purchased from Sigma. LY294002 (PI3K inhibitor) was acquired from Cell Signaling. Primary antibodies are listed in Table 5-1.

JP-153 was synthesized in accordance with the methods devised for orthofunctionalization of aniline derivatives [203]. Briefly, naphthylisocyanate 1 (5.9 mmol, $1.0 \mathrm{~g})$ was added to a solution of t-butylisopropylamine $(5.9 \mathrm{mmol}, 0.9 \mathrm{~mL})$ in diethyl ether $(10 \mathrm{~mL})$ under stirring at room temperature. The colorless solution was stirred for three hours and subsequently cooled to $0^{\circ} \mathrm{C}$. Tetramethylethylenediamine (TMEDA) (12.98 mmol, $2.0 \mathrm{~mL})$ was added followed by n-butyllithium $(11.8 \mathrm{mmol}, 2.43 \mathrm{M}$ in hexanes, $3.0 \mathrm{~mL}$ ). The clear yellow solution was then stirred for three hours, allowing a white precipitate to form. The reaction mixture was cooled to $-78^{\circ} \mathrm{C}$ and aldehyde $2(8.85$ mmol, $1.7 \mathrm{~g})$ in THF $(5 \mathrm{~mL})$ was added dropwise over four minutes. Ethanol $(5 \mathrm{~mL})$ was then added rapidly and the mixture was allowed to warm to room temperature and stirred for one hour. The reaction mixture was then concentrated in vacuo, diluted with dichloromethane (DCM) and washed with saturated ammonium chloride, $\mathrm{NH} 4 \mathrm{Cl}$ (aq.). The organic layer was evaporated onto silica and purified by column chromatography. JP-153 purities were characterized with high-resolution MS and NMR spectroscopy. JP153 and 6-B345TTQ structures and LogP values are presented in Figure 5-2.

\section{Primary retinal endothelial cell culture}

Primary human retinal endothelial cells (RECs, Lot 181; Cell Systems Corp.) were cultured in M131 medium containing MVGS (Invitrogen), gentamicin (10 mg/mL), and amphotericin B $(0.25 \mathrm{mg} / \mathrm{mL})$. Primary cells up to passage six were used. For immunoassays, RECs were plated into six-well plates, cultured for two days, and serum deprived using $0.1 \%$ bovine serum albumin (Sigma) overnight prior to experiments. RECs were pre-treated with inhibitors, SU6656 (1 $\mu \mathrm{M})$, LY294002 (10 $\mu \mathrm{M})$, or JP-153 $(1 \mu \mathrm{M})$ for 1 hour prior to VEGF $(100 \mathrm{ng} / \mathrm{mL})$ stimulation, unless mentioned otherwise. 
Table 5-1. Primary antibodies used in the present chapter.

\begin{tabular}{lcclc}
\hline Antibody & Species of origin & Vendor & Cat. No. & Dilutions \\
\hline VEGF-R2 & $\mathrm{Rb}$ & $\mathrm{CST}$ & $\# 9698$ & $1: 1000$ \\
P-VEGFR2 (Y1175) & $\mathrm{Rb}$ & $\mathrm{CST}$ & $\# 3770$ & $1: 1000$ \\
FAK & $\mathrm{Ms}$ & $\mathrm{BD}$ & $\# 610087$ & $1: 1000$ \\
P-FAK (Y397) & $\mathrm{Rb}$ & $\mathrm{CST}$ & $\# 8556$ & $1: 1000$ \\
P-FAK (Y576/577) & $\mathrm{Rb}$ & $\mathrm{CST}$ & $\# 3281$ & $1: 1000$ \\
P-FAK (Y861) & $\mathrm{Rb}$ & $\mathrm{Abcam}$ & $\# 4804$ & $1: 1000$ \\
P-FAK (Y925) & $\mathrm{Rb}$ & $\mathrm{CST}$ & $\# 3284$ & $1: 1000$ \\
Paxillin & $\mathrm{Ms}$ & $\mathrm{BD}$ & $\# 610051$ & $1: 1000$ \\
P-Paxillin (Y118) & $\mathrm{Rb}$ & $\mathrm{Abcam}$ & $\# 109547$ & $1: 1000$ \\
Akt & $\mathrm{Rb}$ & $\mathrm{CST}$ & $\# 9272$ & $1: 2000$ \\
P-Akt (S473) XP® & $\mathrm{Rb}$ & $\mathrm{CST}$ & $\# 4060$ & $1: 1000$ \\
PARP & $\mathrm{Rb}$ & $\mathrm{CST}$ & $\# 9542$ & $1: 1000$ \\
ERK 1/2 & $\mathrm{Ms}$ & $\mathrm{CST}$ & $\# 9107$ & $1: 1000$ \\
P-ERK 1/2 (T202/Y204) XP® & $\mathrm{Rb}$ & $\mathrm{CST}$ & $\# 4370$ & $1: 2000$ \\
GAPDH XP ${ }^{\circledR}$ & $\mathrm{Rb}$ & $\mathrm{CST}$ & $\# 5174 X P$ & $1: 20,000$ \\
alpha-tubulin & $\mathrm{Ms}$ & LI-COR & $\# 926-42213$ & $1: 20,000$ \\
\hline
\end{tabular}

Notes: Rb: rabbit; Ms: mouse; CST: Cell Signaling Technology; XP: proprietary technology from CST; BD: BD biosciences; 
Structure<smiles>COc1cc(C2CC(=O)Nc3c2cc(Br)c2ccccc32)cc(OC)c1OC</smiles><smiles>COc1cc(C2OC(=O)Nc3c2ccc2ccccc32)cc(OC)c1OC</smiles>

Name

$$
\text { 6-B345TTQ }
$$

$\log P$

$$
\text { JP-153 }
$$

3.56

Figure 5-2. 6-B345TTQ and JP-153 chemical structures and estimated Log P.

Log P estimations were performed in ChemDraw (PerkinElmer, Waltham, MA). 


\section{REC proliferation assays}

To evaluate paxillin-dependent retinal endothelial cell proliferation, 50,000 cells were seeded into each well of a 96-well dish and allowed to adhere overnight. RECs were serum deprived for one hour in $0.1 \%$ BSA, stimulated with VEGF $(100 \mathrm{ng} / \mathrm{mL})$, treated with vehicle, kinase inhibitors or test compounds and incubated for 24 hours. Cellular proliferation was determined using the tetrazolium salt, WST-1 according to the assay manufacturer's instructions (Quick Cell Proliferation Assay Kit II; Abcam, Cambridge, MA). Optical density (O.D.) was measured using a microplate reader at an absorbance of $450 \mathrm{~nm}$. Data represent mean O.D. $\pm \mathrm{SD}, \mathrm{n}=8$ per group. Statistical significance was set at a $\mathrm{P}$-value of $<0.05$. In parallel to the viability experiments, identically treated RECs at 24 hours were incubated with Calcein-AM for 30 minutes and imaged using the EVOS FL ${ }^{\circledR}$ imaging microscope to observe cell numbers.

\section{Annexin-V/FITC staining and flow cytometry analysis for apoptosis}

REC apoptosis was measured by detection of phosphatidylserine translocation to the external surface of the cell membrane [204]. Annexin-V/PI staining was performed according to manufacturer's instructions (BioLegend, San Diego, CA). Briefly, RECs treated with either JP-153 or vehicle for 24 hours were trypsinized and washed twice with ice-cold PBS containing two-percent FBS. Pelleted RECs were resuspended in Annexin$\mathrm{V}$ Binding Buffer at $5.0 \times 10^{6}$ cells $/ \mathrm{mL}$ and incubated with FITC-Annexin-V and propidium iodide (PI) staining solution (BioLegend) at room temperature for 15 minutes in the dark. Cells were then resuspended in binding buffer and analyzed by fluorescence flow cytometry using the BD ${ }^{\mathrm{TM}}$ LSRII Flow Cytometry Analyzer (BD Biosciences, San Jose, CA). Data was statistically assessed using FlowJo analysis software (V10.0.6) (Tree Star Inc., Ashland, OR). Apoptotic cells were defined as Annexin V-positive and PInegative while necrotic cells are defined as Annexin V-positive and PI-positive. Viable cells were considered Annexin-V and PI-negative.

\section{Immunoblot (Western) analysis}

Cellular proteins were analyzed by Western blotting using the Odyssey Sa infrared imaging system as previously described (see Chapter 4) [109]. Lysates were collected in RIPA buffer with protease/phosphatase inhibitors (Roche). Total protein was measured by BCA (Pierce) and processed with LDS buffer containing 2-BME (Sigma). Samples were heated at $70^{\circ} \mathrm{C}$ for 10 minutes, and loaded into Bis-Tris gels (Invitrogen). Immunoblotting was performed with nitrocellulose membranes (Bio-Rad), blocked using Odyssey blocking buffer (LI-COR), and then incubated with primary antibodies overnight at $4^{\circ} \mathrm{C}$. Secondary antibodies against rabbit and mouse (IRDye $800 \mathrm{CW}$ and 680LT) (LI-COR) $[1: 12,500]$ were incubated at $25^{\circ} \mathrm{C}$ for 45 minutes. 


\section{Z'-LYTE SelectScreen ${ }^{\circledR}$ kinase activity assay}

The Z'-Lyte SelectScreen ${ }^{\circledR}$ Single Point biochemical assay was performed by Life Technologies. Percent inhibition was calculated per the Z'-LYTE® Data Analysis protocol (Life Technologies). The concentration of JP-153 used in the reaction mixture was $1 \mu \mathrm{M}$ and ATP was $100 \mathrm{mM}$. Inhibition data represent mean kinase inhibition from two independent runs.

\section{In vitro scratch-wound assay}

REC migration was performed in accordance with methods previously described [205]. RECs (106 cells/well) were seeded to 12-well plates and cultured to confluence. RECs were washed twice with 1X PBS and pre-warmed serum free medium 131 was introduced to wells for one hour to remove any residual effects of supplemented growth factors. Using a sterile $200 \mu \mathrm{L}$ pipette tip, a straight scratch down the center of the well provided the baseline for the analysis and quantification of REC migration and proliferation over 24 hours. Wells were then washed one time with PBS to remove any detached cells. Growth factor supplemented medium with or without JP-153 (0.10$10 \mu \mathrm{M}$ ) was added to each well and plates were immediately imaged using a Cool Snap charge-coupled device (CCD) camera (Roper Scientific, Trenton, NJ) mounted on an Eclipse TE300 inverted microscope (Nikon, Melville, NY). Using $4 \times$ magnification and a computer-controlled stage, images at three specific coordinates per well at the time of the initial wounding were obtained in Metamorph software (Universal Imaging, West Chester, PA). Plates were returned to incubator for 24 hours. The next day, previous coordinates were recalled and images were again collected in Metamorph and then transferred to Adobe Photoshop (CS5 Extended, Ver. 12.1; Adobe Systems, Inc., San Diego, CA). Using the magnetic lasso tool in Photoshop, the outline of protruding/migrating cells from the periphery of the scratch towards the center was measured. The area devoid of migrating cells was recorded and quantified as a percentage change from the previous day's area quantification with the following formula:

$$
\% \text { Area }=\left(1-\frac{A_{24 \text { hours }}}{A_{0 \text { hours }}}\right) * 100
$$

Data represent mean percent wound closure \pm standard deviation (SD). RECs from each group were fixed at 24 hours and stained with DAPI. Fixed cells were then imaged using the EVOS FL Cell Imaging System (Advanced Microscopy Group, AMG; Bothell, WA). A representative image from each experimental group was used to depict extent of wound closure. The characteristic blue hue of DAPI was transposed to white using Photoshop for clarity of cellular nuclei. 


\section{Transwell cellular migration assays}

Cell migration was performed using Transwell ${ }^{\circledR}$ polycarbonate membranes (Corning, Corning, NY) as previously described [206]. Briefly, cell-culture inserts containing membranes $6.5 \mathrm{~mm}$ in diameter and $8.0 \mu \mathrm{m}$ pore size (Corning) were placed in a 24-well tissue culture plate (Corning). The upper surface of the porous membrane was coated with attachment factor at $37^{\circ} \mathrm{C}$ for 1 hour. Human RECs were serum starved overnight in medium 131 containing $0.1 \%$ BSA, trypsinized, pelleted and resuspended in medium 131 with vehicle (0.1\% DMSO) or JP-153 at respective concentrations. RECs were then seeded into the upper chamber at $1 \times 105$ cells/well. Medium 131 containing either vehicle or VEGF $(100 \mathrm{ng} / \mathrm{mL})+/-\mathrm{JP}-153$ was added to the lower chamber. After 24 hours of incubation at $37^{\circ} \mathrm{C}$, non-migrated cells were removed from the upper side of the membrane with cotton swabs and the cells on the lower surface of the membrane were fixed in 4\% paraformaldehyde (PFA) for 15 minutes and washed twice with $1 \mathrm{X}$ PBS. Nuclei were then stained with DAPI (Pierce) in PBS for five minutes and images were collected using the EVOS FL Cell Imaging System (AMG). Images were imported into Adobe Photoshop (Adobe Systems, Inc.) and cells were counted using batch image processing with automation. Briefly, the batches of images from all experimental groups were processed using color correction to enhance DAPI signal against background. Nuclei were outlined using the color-selection tool. The automation protocol was established based on the first image processed in Photoshop to ensure that the processing of each subsequent image was done without any biasing or manipulation of quality and/or integrity. Migrating RECs were quantified from 6 random fields $(n=3)$. Data represent mean number of migrating cells/field $\pm \mathrm{SD}$.

\section{Retinal angiogenesis: murine oxygen-induced retinopathy (OIR) model}

C57BL/6N (Charles River Laboratories; Wilmington, MA) mice were used in all experiments. All animal studies were performed under the guidelines of the Association for Research in Vision and Ophthalmology for the humane use of animals in vision research, and under the guidance and approval of the Institutional Animal Care and Use Committee at the University of Tennessee Health Science Center.

Retinal angiogenesis was induced using a mouse model of oxygen-induced retinopathy (OIR), as previously described [109, 161]. Five independent litters on three separate occasions were used for OIR experiments. Mouse pups exposed to the oxygen chamber were shuffled into three groups prior to dosing (P12) in order to provide intralitter controls. Experimental groups were as follows: 1) mice reared in normal atmospheric conditions (negative-control; normoxia) 2) mice exposed to OIR/hyperoxic chamber and treated with vehicle nanoemulsion $(1 \mu \mathrm{L} / \mathrm{g}$; positive-control $) ; 3)$ OIR-mice treated with JP-153-loaded nanoemulsion at $0.5 \mathrm{mg} / \mathrm{kg}$ and 4) JP-153 at $5.0 \mathrm{mg} / \mathrm{kg}$. Mouse pups were exposed to $75 \%$ oxygen at post-natal day seven (P7) for five days and then returned to normal oxygen (P12). Ocular nanoemulsion used for drug delivery comprised Capryol 90 (10.5\% v/v), Triacetin (10.5\% v/v), Tween-20 (24.5\% v/v) and Transcutol P (24.5\% v/v) (Gattefossé Pharmaceuticals, Saint-Priest, France) generated 
via homogenization and water titration methods, as previously described [109]. JP-153 was first loaded into the oil-phase and then incorporated into the final ME formulation and stored at room temperature away from light until dosing. OIR mice were weighed prior to receiving each daily dose to both eyes using either JP-153 or vehicle-loaded nanoemulsion from P12 to P17 (Vehicle control, N=8; JP-153 $0.5 \mathrm{mg} / \mathrm{kg}, \mathrm{N}=14$; JP$1535.0 \mathrm{mg} / \mathrm{kg}, \mathrm{N}=14$ ). On P17, retinas were removed, dissected, mounted, and stained for endothelial cells to investigate retinal angiogenesis. At the conclusion of the study, anesthetized animals were humanely euthanized according IACUC guidelines.

\section{Retinal whole-mount imaging and analysis}

Enucleated whole-eyes from P17 mouse pups underwent immediate weak fixation in 4\% paraformaldehyde (PFA) in PBS for one hour and washed three times in ice-cold PBS. Retinas were carefully isolated under a Leica S6E dissecting stereomicroscope (Leica Microsystems, Buffalo Grove, IL) and mounted onto microscope slides. Whole retinas were incubated overnight at $4^{\circ} \mathrm{C}$ with isolectin B4-594 (Alexa Fluor 594; Molecular Probes, Eugene, OR) as previously described [109, 164]. Isolectin-stained retinas were then washed three times in 1X PBS and sealed under coverslips using Vectashield mounting medium (Vector Laboratories, Inc.) and stored at $4^{\circ} \mathrm{C}$ until imaging.

Images were acquired using a Zeiss LSM 710 system attached to a Zeiss Axio Observer inverted microscope with Zen 2010 v.6.0 software (Carl Zeiss Microscopy). Multi-dimensional acquisition was carried out using Z-stacks with $<4 \mu \mathrm{m}$ slicing intervals and tile-scan automation with an $8 \%$ tile-overlap at a resolution of at least $512 \mathrm{x}$ 512 pixels per tile and digitally stitched together. Quantification of avascular area (AV) and neovascularization (NV) in retinal whole mounts was performed in Adobe Photoshop (Adobe Systems, Inc.) as previously described [109]. The area devoid of vascularization around the optic disc was characterized as percentage of total retinal area $(\% \mathrm{AV})$. Photoshop color-range analysis tool were used to outline NV formations after intensity thresholds were set to exclude normal vasculature. Data was recorded as a percentage of total retinal area $(\% \mathrm{NV})$. Representative whole-mounted retinas were displayed using the exact quantified outlined areas and layered back into place onto the original whole-retina image. Using the linear light blending method in Photoshop, both avascular and neovascular areas were transposed in white.

\section{In silico molecular docking}

Docking calculations were performed in Molecular Operating Environment software (MOE 2012, ver. 2014.09, Chemical Computing Group, Ltd., Montréal, Canada). The NMR solution structure of paxillin in complex with FAK was obtained from the Protein Data Bank, PDB entry: 2L6F. Missing atomic coordinates and protonation states of the amino acid residues within the peptide were assigned using the "Structure Preparation" and "Protonate 3D" facilities in MOE. The coordinates of water 
molecule's hydrogen atoms were assigned to optimize hydrogen bonds with neighboring molecules/residues. The DOCK facility in MOE was used to predict potential binding modes of JP-153. The bound paxillin was deleted from the model, allowing "ligands" to access binding pockets on the alpha-helices H1/4 and H2/3 surfaces of FAT, corresponding to interactions with LD2 and LD4, respectively. Ligands were docked in an induced fit" scheme allowing side chain flexibility on side chain's residues and predicted binding free energies were assessed using MOE's GBVI/VSA dG scoring function. Two docking strategies were used: i) an "agnostic" approach where ligands are free to potentially dock anywhere on the FAT receptor's surface in the regions corresponding to receptor:paxilin interactions; and ii) constraining potential binding sites to be in specific locations on the receptor's surface [207].

Enantiomers of chiral drugs may show significant pharmacological differences due to chemical confirmations within proposed target engagement sites. Thus, the FDA recommends that the activity of individual enantiomers should be characterized for their principal pharmacologic effects [208]. Since JP-153 is racemic, we built both the R- and $\mathrm{S}$ - enantiomers into MOE for molecular docking simulations. The best-scored binding modes for JP-153 were used in the three-dimensional reconstructions of isomer binding to the FAT domain in MOE.

\section{Protein thermal shift assay}

Protein thermal shift assays were performed as described previously [209]. Focal adhesion targeting domain (FAT) of FAK ( $\Delta$ 902; Santa Cruz; sc-4107) was used at concentrations $0.125-1.0 \mathrm{mg} / \mathrm{mL}$ for initial melting temperature determination, and at $0.5 \mathrm{mg} / \mathrm{mL}$ for JP-153 binding experiments. Protein melt curves were performed using a protein melt buffer (PMB) comprising $0.1 \%$ CHAPS $(110 \mathrm{mM} \mathrm{NaCl}, 50 \mathrm{mM}$ Tris $\mathrm{HCl}, 5$ $\mathrm{mM}$ EDTA) at a $\mathrm{pH}$ of 8.0 at $4{ }^{\circ} \mathrm{C}$. Reaction solutions of deionized water, PMB containing FAT proteins, ligand (0.1\% DMSO vehicle or JP-153) and SYPRO ${ }^{\circledR}$ Orange $(5 \mathrm{X})$ were added to 96 -well plates (MicroAmp ${ }^{\circledR} 0.1 \mathrm{~mL}$ optical reaction plates; Applied Biosystems, Foster City, CA) at a final volume of $20 \mu \mathrm{L}$. Plates were kept on ice until placed into the StepOnePlus ${ }^{\mathrm{TM}}$ Real Time PCR machine (Applied Biosystems) whereupon the reaction was started and held at $25{ }^{\circ} \mathrm{C}$ for two minutes and then ramped to $99{ }^{\circ} \mathrm{C}$ at $0.5^{\circ} \mathrm{C} /$ minute increments over 2.5 hours to generate melting curves. Fluorescent readings were, normalized to background fluorescence obtained from wells without protein, exported to Sigmaplot and analyzed using dynamic curve fitting tools to calculate melting temperatures $(\mathrm{Tm})$. Reactions were performed in duplicate.

\section{Statistical analyses}

All statistical analyses were performed in SigmaPlot 12.0 software (Systat Software, Inc., San Jose, CA). Data represented herein were performed in replicates of three or more and presented as the mean \pm standard deviation (SD), unless otherwise indicated. Differences among groups were analyzed using one-way analysis of variance 
(ANOVA). When overall analysis revealed significance among groups, means were compared and tested using Tukey's post-hoc analysis. Statistical significance was set at $\mathrm{P}$ $<0.05$. P-values representing significance $<0.05,0.01$, and 0.001 are denoted with symbols $* * *$, and $* * *$, while significance $<0.05,0.01$, and 0.001 among treatment arms are represented with $\dagger, \dagger \dagger$, and $\dagger \dagger \dagger$, respectively.

\section{Results}

\section{Src/FAK-paxillin signaling pathway in REC proliferation}

FAK and paxillin are coordinators of FA turnover during VEGF-induced proliferation and migration - two seminal events of angiogenesis [76]. In order to confirm the relevance of these two players in VEGF-induced proliferation of primary retinal endothelial cells (REC), we stimulated RECs with VEGF and analyzed cell lysates for FAK and paxillin phosphorylation over time (Figure 5-3). Recombinant human rhVEGF (100 ng/mL) activates VEGF receptor-2 (VEGFR-2) with maximal phosphorylation occurring within 15 minutes at a major phosphorylation site, Tyr-1175 (Figure 5-3A). Activation of VEGFR-2 triggers autophosphorylation of FAK Y397 (*P $<0.05, * * * \mathrm{P}<0.001$ ) which promotes association of Src with FAK [210] and subsequently leads to Src-dependent FAK phosphorylation of its kinase domain loop, Y576/577 and focal adhesion targeting (FAT) domain, Y925. Src-dependent activation and binding of FAK forms the Src/FAK focal adhesion complex (FAC) which phosphorylates paxillin $\mathrm{Y} 118$ (Figure 5-3B, ${ }^{* *} \mathrm{P}<0.01, * * * \mathrm{P}<0.001$ ).

To determine if the Src/FAK complex is necessary for paxillin activation in RECs and thus proliferation, we examined FAK and paxillin phosphorylation in VEGF stimulated RECs treated with Src-kinase inhibitor, SU6656 $(1 \mu \mathrm{M})$ [211]. In Figure 5-4, we show that inhibiting Src kinase reduces the phosphorylation of FAK Y576/577, Y925 and Y861 (††P $<0.01)$, but does not affect autophosphorylation of Y397. An inactive Src/FAK complex fails to phosphorylate paxillin Y118 (Figure 5-4A, $\dagger \dagger \mathrm{P}<0.01$ ). We again treated RECs with SU6656 for 24 hours and showed that inhibition of Src-mediated phosphorylation of FA proteins leads to a significant decrease in VEGF-induced proliferation (Figure 5-4B, $\dagger \dagger \dagger \mathrm{P}<0.001$ ).

\section{Discovery of JP-153 as a potent inhibitor of VEGF-induced proliferation}

Src-dependent FAK and paxillin phosphorylation correlated with VEGF-induced proliferation in RECs. We used this phenotypic response to derive compounds related to a known paxillin protein disruptor, 6-B345TTQ [117]. Our initial lead identification efforts yielded the analog JP-153, which was $\sim 50$ times more potent than 6-B345TTQ in REC proliferation assays (Figure 5-5A, $† \mathrm{P}<0.05$, $\dagger \dagger \dagger \mathrm{P}<0.001$; Figure 5-5B, $\dagger \dagger \dagger \mathrm{P}<$ $0.001)$. JP-153 and 6-B345TTQ analyses revealed $\mathrm{IC}_{50}$ of 1.42 and $58.2 \mu \mathrm{M}$, respectively. We used Calcein-AM staining (Figure 5-5C) to show that live cell number 
(A.) VEGF $(100 \mathrm{ng} / \mathrm{mL})$

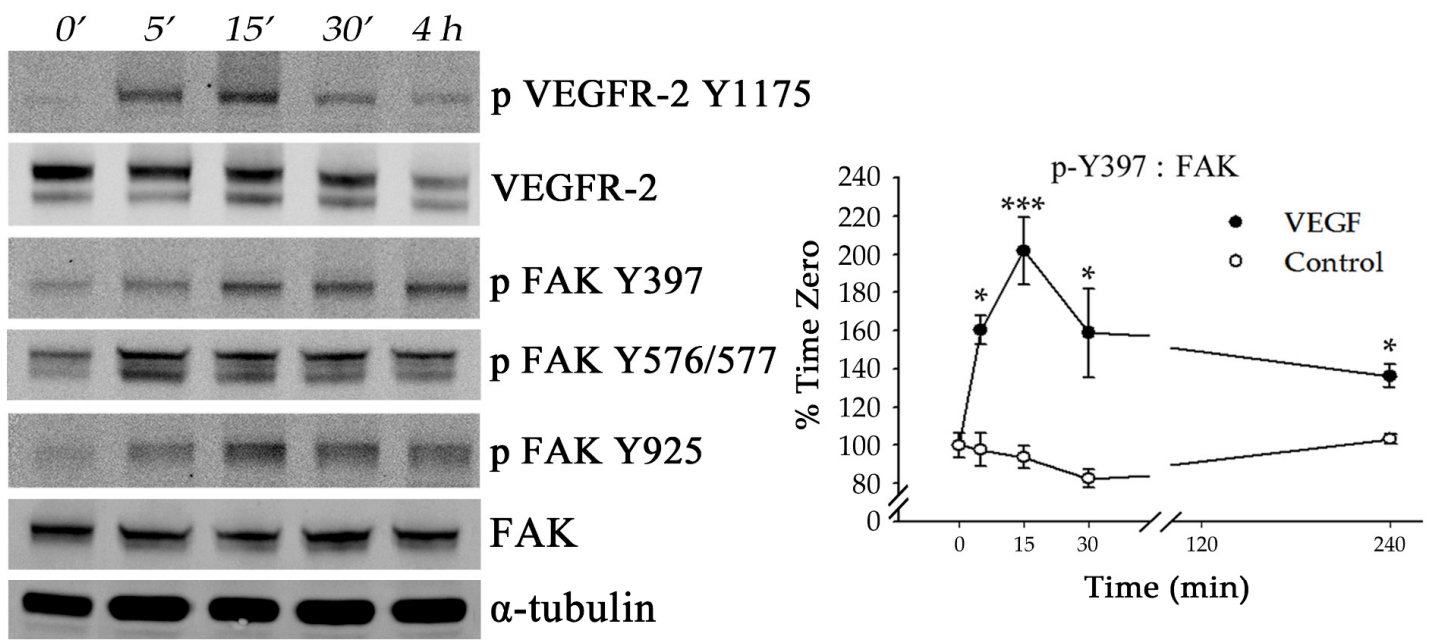

(B.)
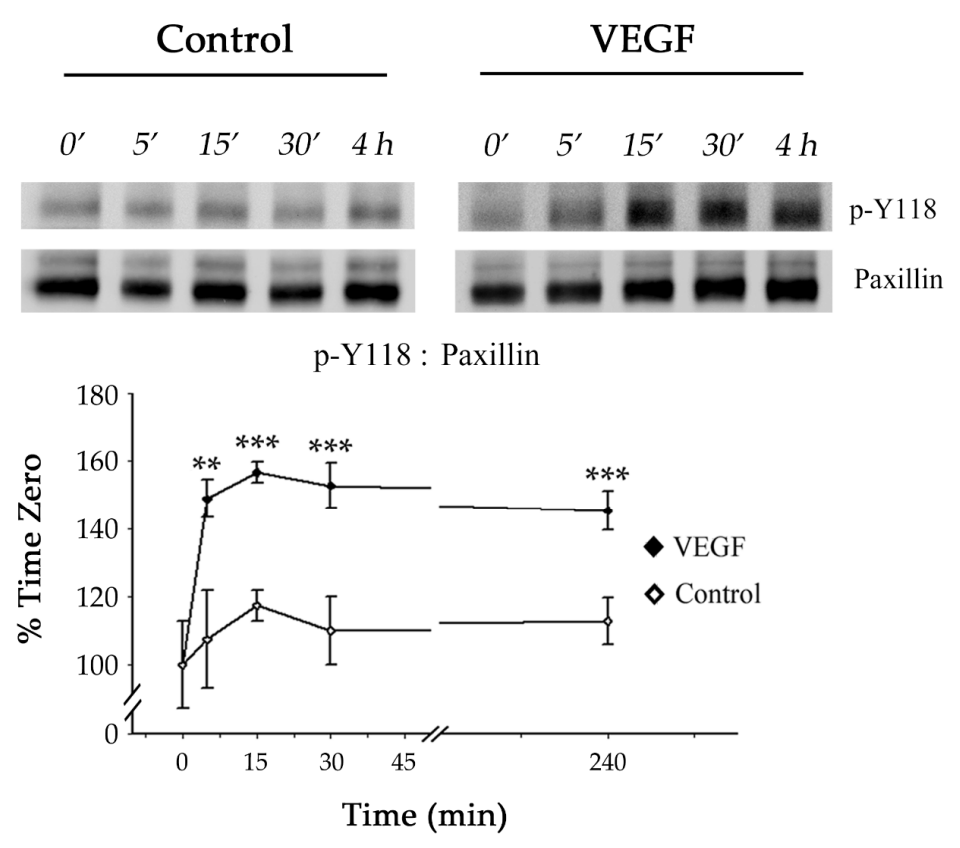

Figure 5-3. VEGF-induced FA signaling in RECs.

A) Retinal endothelial cells were stimulated with VEGF (100 ng/mL) and cellular lysates were collected over four hours and focal adhesion protein activation was measured using Western blotting as described in Materials and Methods. Initially, VEGFR-2 is activated at Y1175 upon VEGF ligation which triggers FAK Y397 autophosphorylation (representative Western blots on the left, analysis of FAK pY397 levels on the right) (*P $<0.05, * * * \mathrm{P}<0.001)$. Subsequently, Src-kinase binds to FAK and further activates the kinase-domain loop, FAK Y576/577 and the FAT domain, FAK Y925. B) Src-dependent activation of FAK coincides with paxillin Y118 phosphorylation over four hours $(* * \mathrm{P}<$ $0.01, * * * \mathrm{P}<0.001)$. Data represent mean $\pm \mathrm{SD}, \mathrm{n}=4-8$. 
(A.)
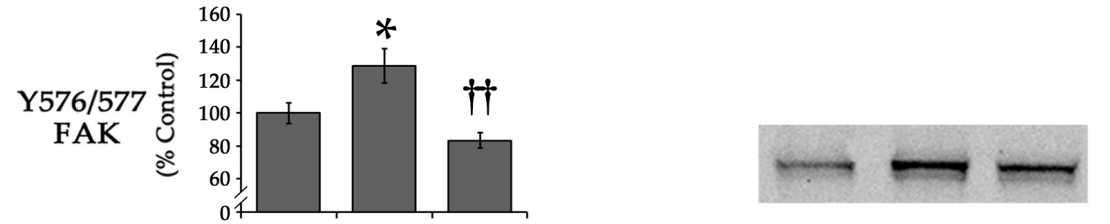

p Y397
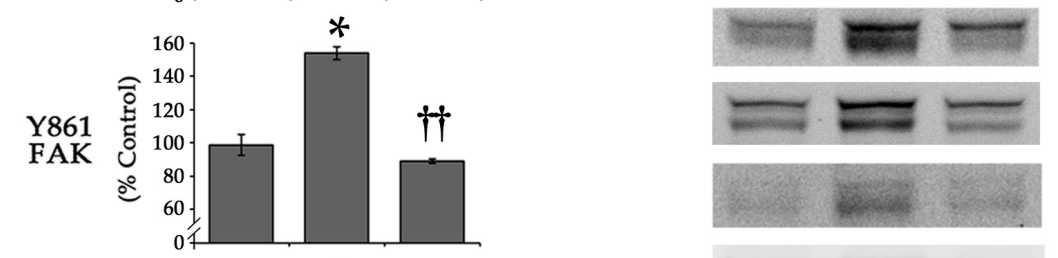

p Y $576 / 577$
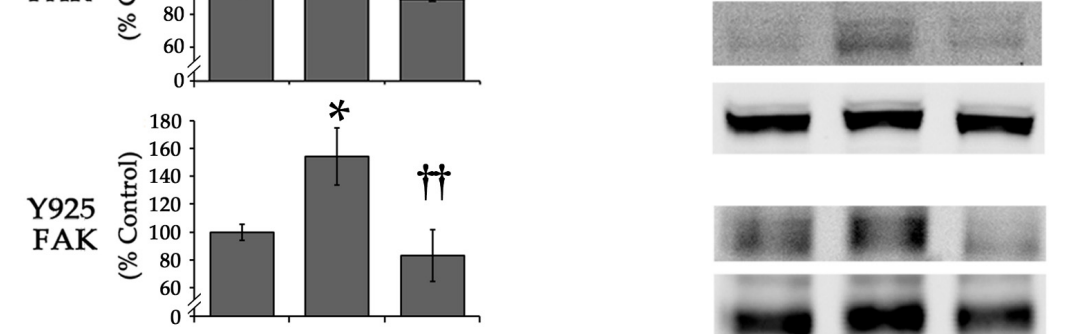

p. Y861

p Y925

$\square$ FAK

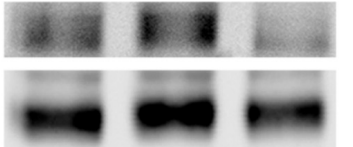

p Y118

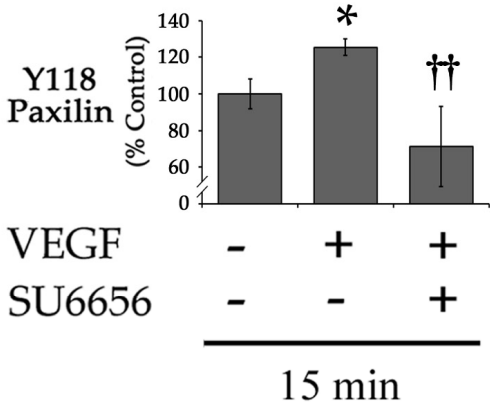

$\propto \alpha$-tubulin

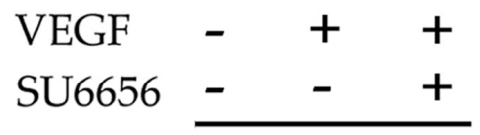

$15 \min$

(B.)

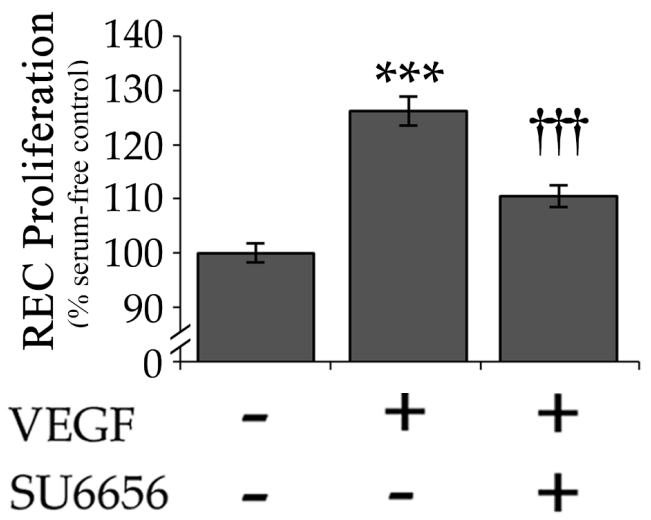

Figure 5-4. Src-dependent activation of FAK and paxillin in RECs.

A) Src-inhibition with SU6656 $(1 \mu \mathrm{M})$ inhibited VEGF's activation of FAK Y 576/577, Y861 and Y925 and paxillin Y118 $(* \mathrm{P}<0.05, \uparrow \uparrow \mathrm{P}<0.01)$ but did not prevent autophosphorylation of FAK Y397 $(\mathrm{P}>0.05)$. Data $(\mathrm{n}=3)$ represent mean $\pm \mathrm{SD}$. B) VEGF-mediated proliferation of RECs was performed as described in Materials and Methods. VEGF-induced proliferation in RECs was reduced in the presence of SU6656 $(1 \mu \mathrm{M})$ which correlated with FA activation $(* * *, \uparrow \dagger \uparrow \mathrm{P}<0.001)$. (Data represent mean \pm $\mathrm{SD}, \mathrm{n}=8)$. 
(A.)

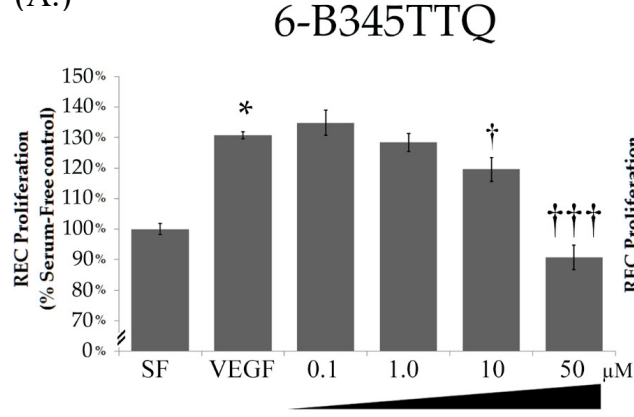

(B.)

$$
\text { JP - } 153
$$

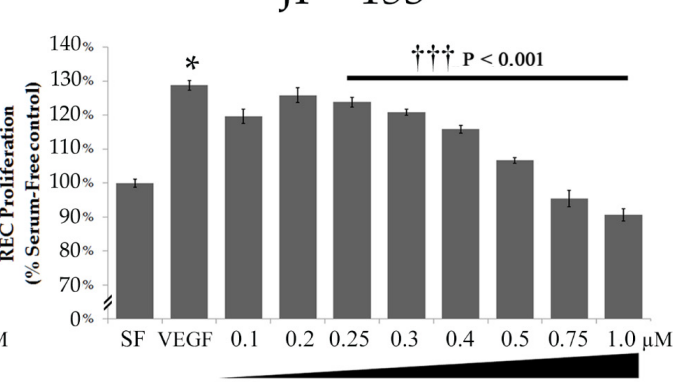

(C.)
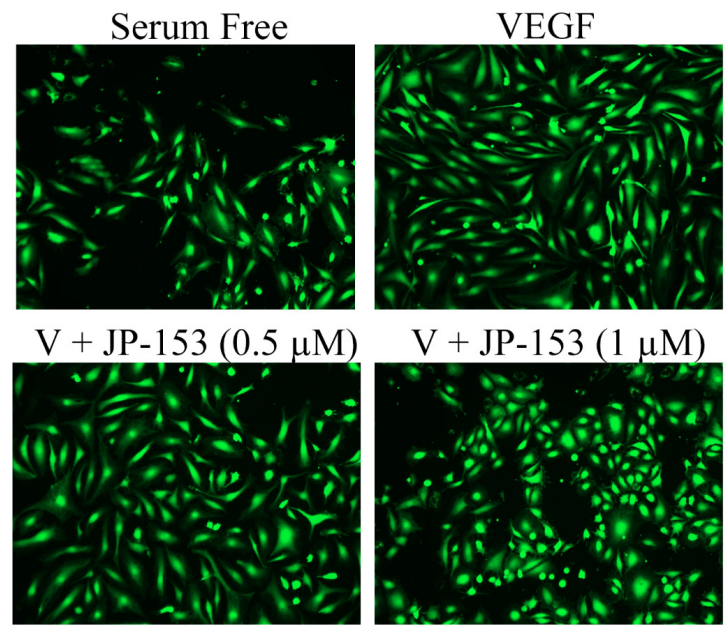

(D.)

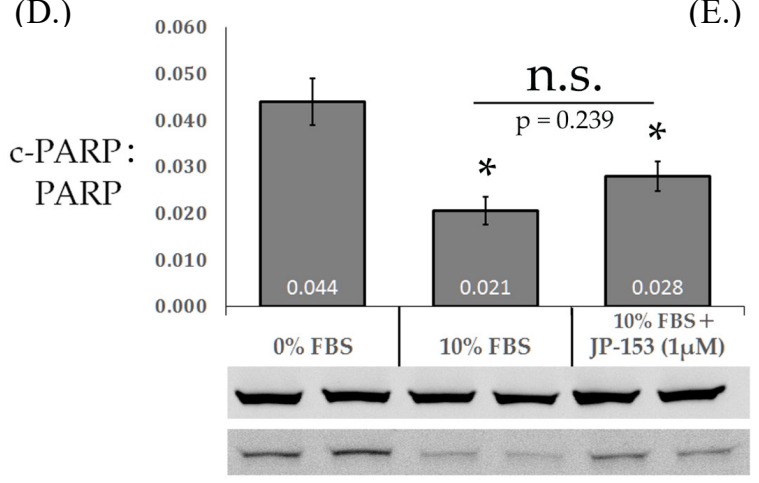

(E.)

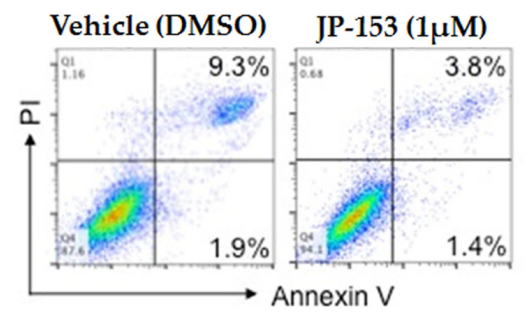

Figure 5-5. Discovery of JP-153 as an inhibitor of VEGF-induced proliferation.

A) 6-B345TTQ, a known paxillin disruptor inhibited REC proliferation at concentrations greater than $10 \mu \mathrm{M}(\uparrow \mathrm{P}<0.05, \uparrow \uparrow \uparrow \mathrm{P}<0.001)$. B) Due to potency issues, redesigned derivative, JP-153 inhibited REC proliferation substantially with concentrations as low as $0.25 \mu \mathrm{M}(\dagger \dagger \uparrow \mathrm{P}<0.001)$. Data represent mean $\pm \mathrm{SD}, \mathrm{n}=8$. C) We observed cell numbers using Calcein-AM. D) We investigated apoptosis using cleaved-PARP signaling in Western blots and showed that JP-153 $(1 \mu \mathrm{M})$ did not significantly enhance apopototic signaling ( $\mathrm{P}=0.239$ vs. $10 \%$ FBS controls; $\mathrm{n}=3$ ). E) Flow cytometry quantified apoptotic cells within the population treated with JP-153 $(1 \mu \mathrm{M}, 24 \mathrm{~h}$ to confirm that cell death was not induced with treatment, as compared to controls $(n=50,000$ cells $)$. 
is reduced with JP-153 treatments in addition to reduced proliferative activity. Yet, JP153 does not promote apoptosis in cells, as characterized by cleavage of PARP (Figure 5-5D) and Annexin V/PI staining of JP-153 treated RECs (24 hours) (Figure 5-5E).

\section{Effector signaling through an activated Src/FAK-paxillin complex is Akt-dependent}

We postulated that JP-153 inhibits REC proliferation through disruptions in FA protein interactions, as shown by Kummer et al. with 6-B345TTQ [117]. Thus, we treated RECs with JP-153 $(1 \mu \mathrm{M})$ for one hour and then stimulated them with VEGF for four hours. In cells JP-153 significantly reduces Y118 phosphorylation (Figure 5-6, **, $\dagger$ P $<$ 0.01 ), but did not inhibit constitutive levels of unstimulated RECs treated with JP-153 (p $=0.749$ ). Next, we examined downstream FA effector signaling during early VEGF activation at 15 minutes (Figure 5-7). We pre-treated RECs with JP-153 for one hour prior to VEGF-activation and measured phosphorylation of FAK phosphorylation sites, as well as downstream angiogenic markers AKT and ERK. Our results again confirmed that JP-153 reduces activation of paxillin Y118 compared to VEGF controls $\left(^{*}, \dagger \mathrm{P}<\right.$ 0.05), but does not change autophosphorylation of FAK Y397; these results mimic the activity of SU6656 ( †† P< 0.01). However, when we probed for FAK Y 576/577, Y861, and Y925 in cells, JP-153 did not affect levels of Src-dependent FAK phosphorylation sites $(\mathrm{P}>0.05)$, while SU6656 inhibited these levels strongly ( $\dagger \mathrm{P}<0.05$, $\dagger \mathrm{P}<0.01)$.

Activation of paxillin Y118 primes the complex for interactions with signaling proteins PI3K and MEK which facilitates the activation of their downstream substrates, AKT and ERK, respectively to promote cytoskeletal rearrangements during proliferation and migration [212-214]. We compared RECs treated with JP-153 and SU6656 with those treated with PI3K inhibitor, LY294002 $(10 \mu \mathrm{M})$ (Figure 5-7). Both p-ERK 1/2 and p-Akt (Ser473) levels rose under VEGF, but only Akt was effectively blocked by SU6656 and JP-153 (*, $\uparrow \mathrm{P}<0.05, \dagger \uparrow \mathrm{P}<0.01)$. Neither show significant inhibition of $\mathrm{p}$ ERK $1 / 2$ at concentrations tested $(\mathrm{P}>0.05)$. However, complete inhibition of Akt phosphorylation by LY294002 caused no reductions in FAK or paxillin activation, suggesting the Src/FAK/paxillin activation cascade precedes PI3K-induced Akt phosphorylation. However, unlike JP-153 or SU6656, LY294002 significantly induced ERK activation ( $\dagger \mathrm{P}<0.05$; LY294002 vs. VEGF). To validate an Akt-dependent proliferation pathway, cells treated with LY294002 potently inhibited proliferation, with levels far exceeding serum starvation, Src-inhibition and JP-153 treatments $(* * *, \dagger \dagger \uparrow \mathrm{P}<$ 0.001). Together, these data suggest JP-153 acts to inhibit REC proliferation through an Akt-dependent but ERK-independent mechanism. To rule out kinase inhibition, we show that JP-153 was not a direct kinase inhibitor of FA signaling effectors per se, as measured by the Z'-LYTE SelectScreen ${ }^{\circledR}$ single-point biochemical assay (Table 5-2). 

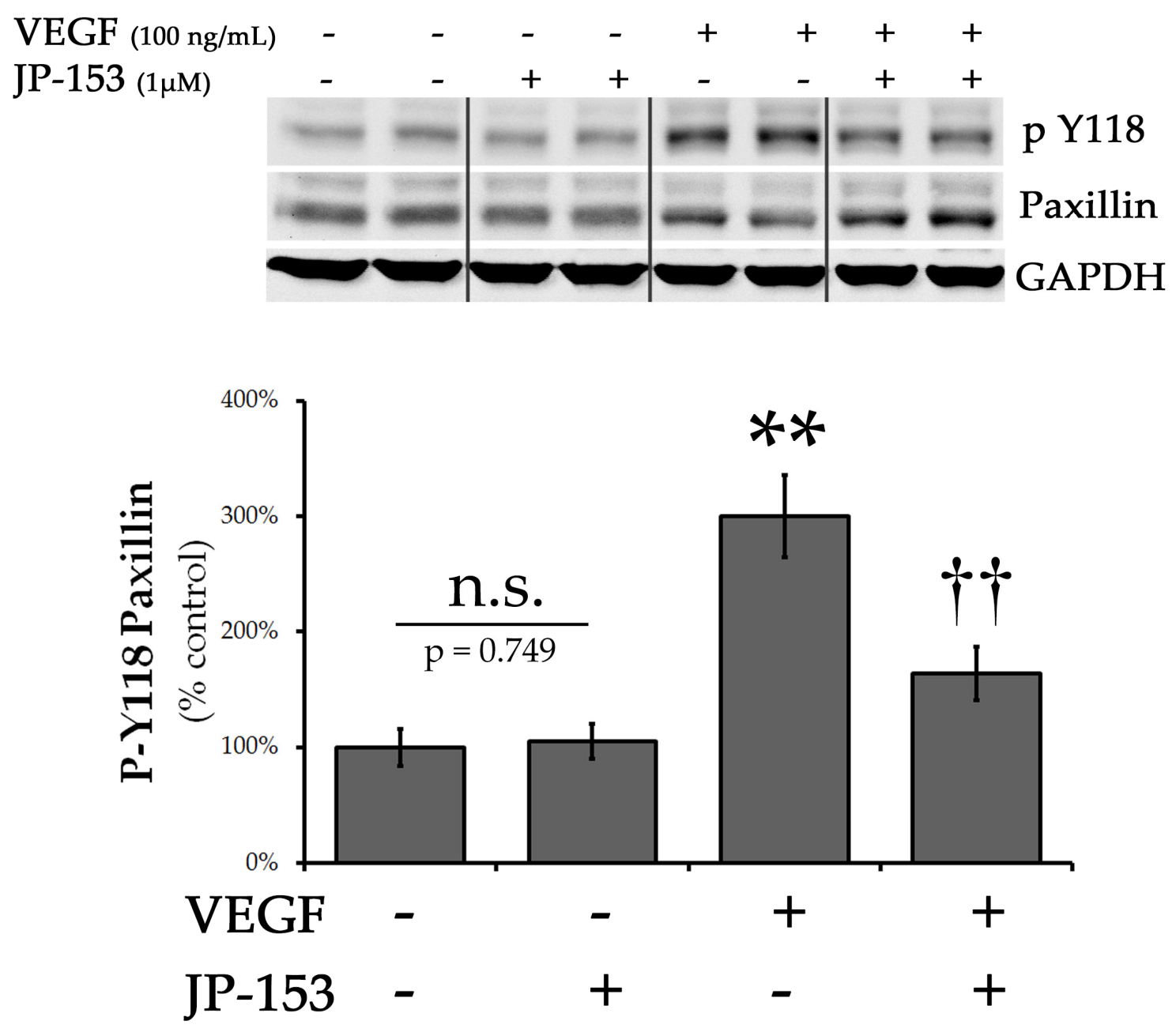

Figure 5-6. JP-153 inhibits VEGF-induced activation of paxillin Y118.

REC lysates were collected at four hours post-VEGF activation and phosphorylation of paxillin Y118 was measured using Western blotting (top panel). JP-153 significantly reduced phosphorylation in cells stimulated with VEGF $(* *, \uparrow \uparrow \mathrm{P}<0.01)$, but did not affect constitutive/unstimulated levels ( $\mathrm{P}=0.749$ vs. vehicle control) (bottom panel). Data represent mean $\pm \mathrm{SD} ; \mathrm{n}=3$. 


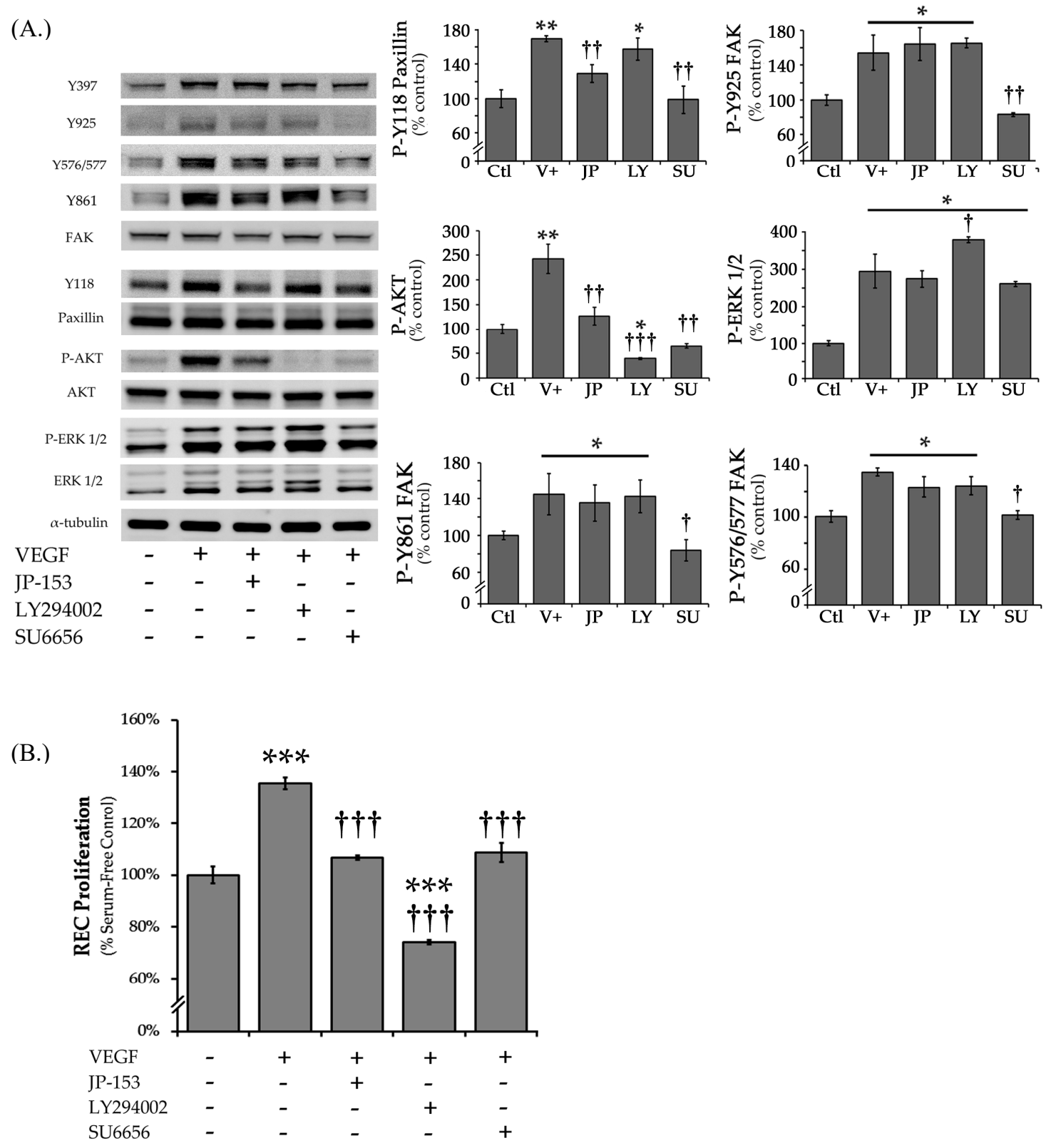

Figure 5-7. JP-153 inhibits proliferation through Src/FAK/paxillin FA complex.

A) RECs activated by VEGF (100 ng/mL for $15 \mathrm{~min}$ ) show FA and effector signaling after $1 \mathrm{~h}$ pre-treatments with JP-153 $(1 \mu \mathrm{M})$, SU6656 $(1 \mu \mathrm{M})$ or LY294002 $(10 \mu \mathrm{M})$. JP153 and SU6656 significantly reduced paxillin Y118 phosphorylation $(* *, \uparrow \uparrow \mathrm{P}<0.01)$, SU6656 inhibits pFAK Y576/577 (*,† P < 0.05), Y861 (*,†P<0.05), and Y925 (*P < $0.05, \uparrow \uparrow \mathrm{P}<0.01)$. VEGF-induced pAKT (S473) phosphorylation was inhibited by JP153, SU6656 and LY294002 (**, $\uparrow \uparrow P<0.01, \uparrow \dagger \uparrow P<0.001)$. Neither SU6656 nor JP-153 affected pERK-1/2 (P>0.05), but LY294002 increased pERK $(\uparrow \mathrm{P}<0.01$ vs. VEGF controls). B) REC-proliferation is dependent on AKT, as LY294002 resulted in potent inhibition of proliferation more than JP-153 or SU6656 (***, $\uparrow \dagger \uparrow \mathrm{P}<0.001, \mathrm{n}=8)$. 
Table 5-2. Z-LYTE SelectScreen ${ }^{\circledR}$ kinase inhibitor assay.

\begin{tabular}{lc}
\hline Kinase & \% Inhibition $\neq$ \\
\hline ABL1 & 4 \\
EGFR & 0 \\
FLT (VEGFR1) & -2 \\
FLT3 & 7 \\
FLT4 (VEGFR3) & 4 \\
mTOR (FRAP1) & 4 \\
FYN & 6 \\
HCK & 3 \\
KDR (VEGFR2) & -3 \\
PTK2 (FAK) & 1 \\
PTK2B (FAK2) & 1 \\
Src & 4 \\
Yes & 1 \\
\hline
\end{tabular}

Notes: ¥Percent inhibition was calculated according to the Z'-LYTE Data Analysis protocol (Life Technologies). 


\section{Paxillin modulation with JP-153 inhibits VEGF-induced migration of retinal endothelial cells}

We have shown that JP-153 inhibited REC proliferation through disruptions in Src/FAK activation of paxillin Y118, and pAkt (Figure 5-7). Since angiogenesis requires two distinct but cooperative mechanisms, proliferation and migration, we examined JP153 effect on migration using the standard scratch wound assay. VEGF-induced REC migration was significantly inhibited in JP-153 treatments over a range of concentrations $(0.10$ to $10 \mu \mathrm{M})$ (Figure 5-8; ${ }^{*}, \dagger \mathrm{P}<0.05$, $\left.\dagger \dagger \dagger \mathrm{P}<0.001\right)$. However, it is difficult to ascertain whether proliferative mechanisms are also contributing to wound closure under this assay. Therefore, we validated our scratch-wound results with the transwell migration/invasion assay using VEGF as the chemotactic inducer [215] in order to distinguish migration activity from proliferation induced by growth factors. Our results show that JP-153 inhibits REC invasion at sub-micromolar concentrations $(0.10-0.50$ $\mu \mathrm{M}$ ) (Figure 5-9, $* * *, \dagger \dagger+\mathrm{P}<0.001)$ suggesting that JP-153 modulates both proliferation and migration of RECs by disrupting the activation and signaling of paxillin.

\section{Molecular docking calculations suggest that JP-153 binds preferentially to FAT, the paxillin-binding subdomain of FAK}

We hypothesized that one of the binding preferences of JP-153 was located between FAK and paxillin where the focal adhesion targeting (FAT) domain of FAK interacts with the leucine-rich domains (LD-2/4) of paxillin to regulate signaling [216, 217]. Using agnostic docking calculations in Molecular Operating Environment (MOE) software, JP-153 preferred the hydrophobic binding pocket comprising the alpha $(\alpha)-\mathrm{H} 1$ and $-\mathrm{H} 4$ of FAT, while the $\mathrm{H} 2 / \mathrm{H} 3$ interface, or any other position around the FAT domain was limited to JP-153 binding (Figure 5-10). We investigated the docking poses of both the R and S enantiomers of JP-15 (one-chiral center) within dummy atoms sites identified in MOE software tools that corresponded with the $\mathrm{H} 1 / \mathrm{H} 4$ interface. Our results showed that both isomers of JP-153 could bind to the hydrophobic pocket in similar ways, though with slightly different orientations of the naphthalene and tri-methoxy ring systems (see Figure 5-2 for structures). The 3D models in Figure 5-11 shows how both R- and S-isomers of JP-153 might be positioned between the FAT domain of FAK and the LD2 domain of paxillin. Ligand interactions of R and S enantiomers of JP-153 revealed potential bonds between amino acid residues Isoleucine 936, Leucine 1021 and Histidine 1025 located within the $\mathrm{H} 1 / \mathrm{H} 4$ pocket. Ligand interaction feature in MOE showed that for the R-JP-153 enantiomer, an arene-arene bond between the imidazole ring of His 1025 and JP-153's tri-methoxy ring system exists (Figure 5-11C). Residue Ile936 appears to interact with JP-153's naphthalene ring system, and this was true for both enantiomers. The S-enantiomer, however differed slightly from the R configuration with the tri-methoxy ring's arene-hydrogen bond interacting with both amino acid residues, Leu 1021 and His 1025 (Figure 5-11D). 
(A.)

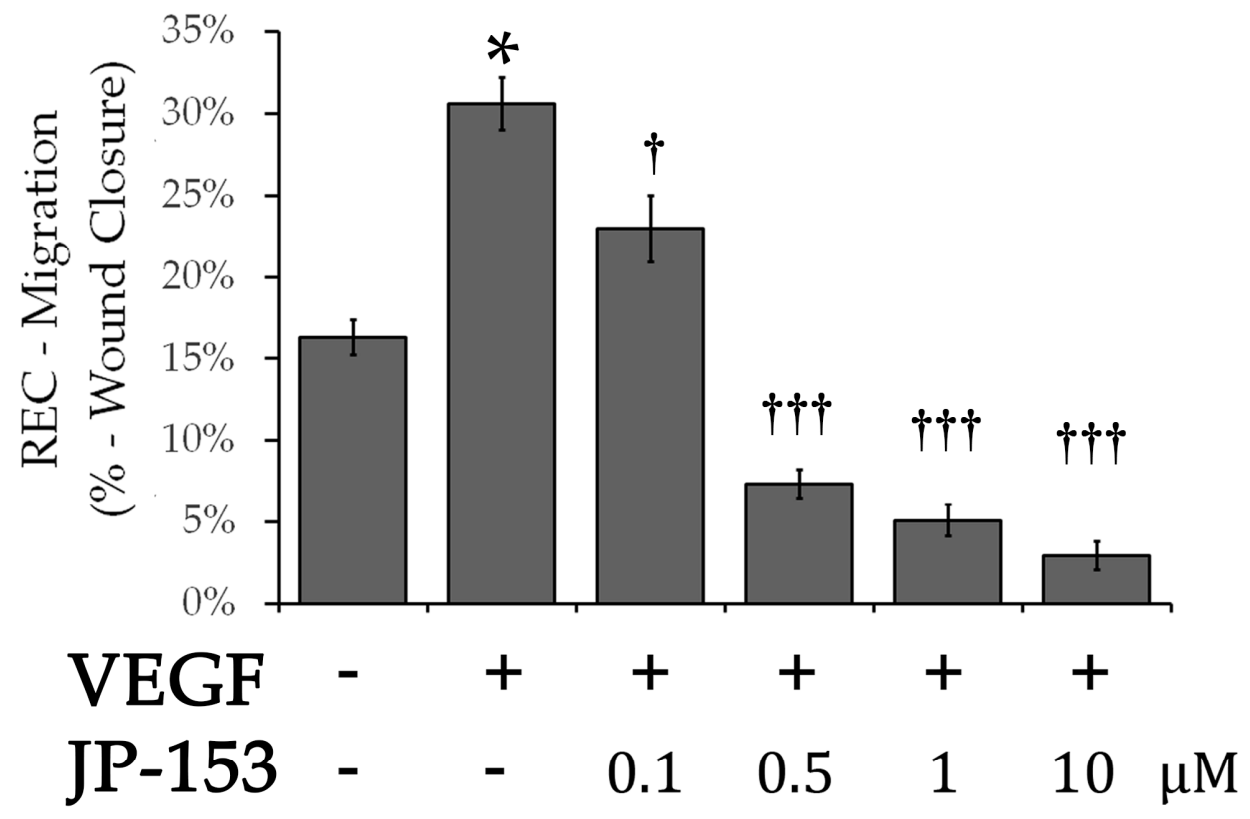

(B.)

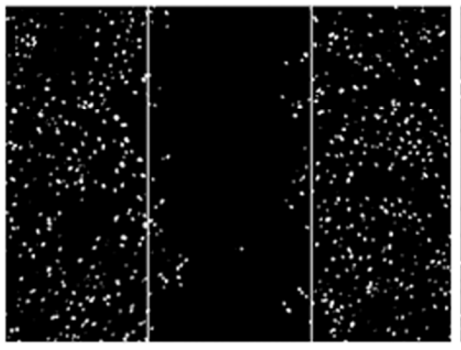

Control

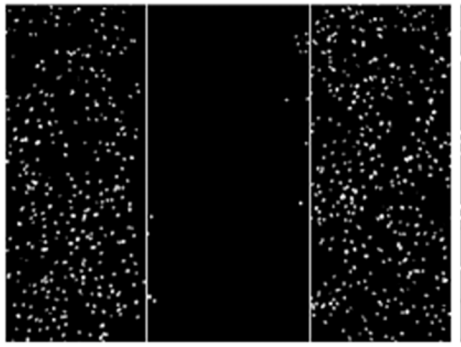

$\mathrm{V}+0.5 \mu \mathrm{M}$

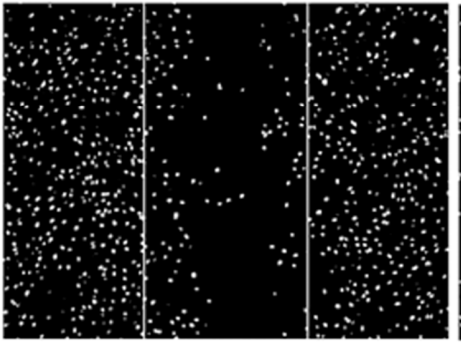

VEGF

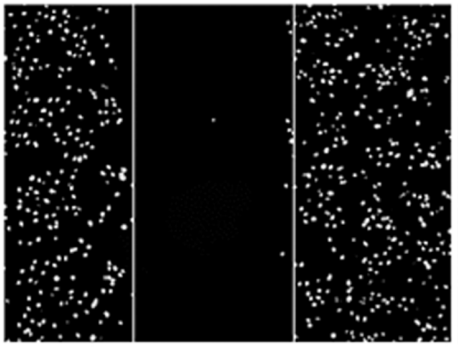

$\mathrm{V}+1 \mu \mathrm{M}$

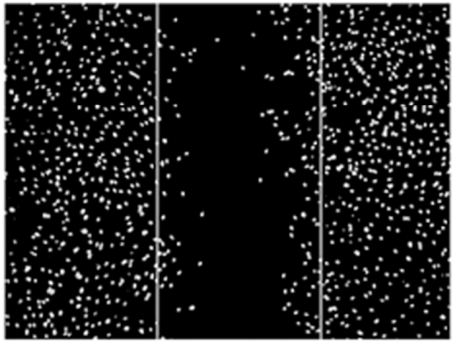

$\mathrm{V}+0.1 \mu \mathrm{M}$

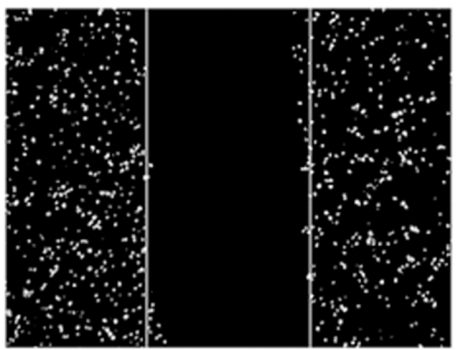

$\mathrm{V}+10 \mu \mathrm{M}$

Figure 5-8. JP-153 inhibited VEGF-induced REC migration.

The scratch-wound migration assay was performed in RECs exposed to VEGF for 24 hours. JP-153 inhibited VEGF-induced migration in a concentration dependent manner (data analysis in panel $A$; representative DAPI-stained images after 24 hours is shown in panel $B$ ). (Data are presented as the mean $\pm \mathrm{SD} ; \mathrm{n}=6$; ${ }^{*}, \uparrow \mathrm{P}<0.05, \uparrow \uparrow \uparrow \mathrm{P}<0.001$ ). 


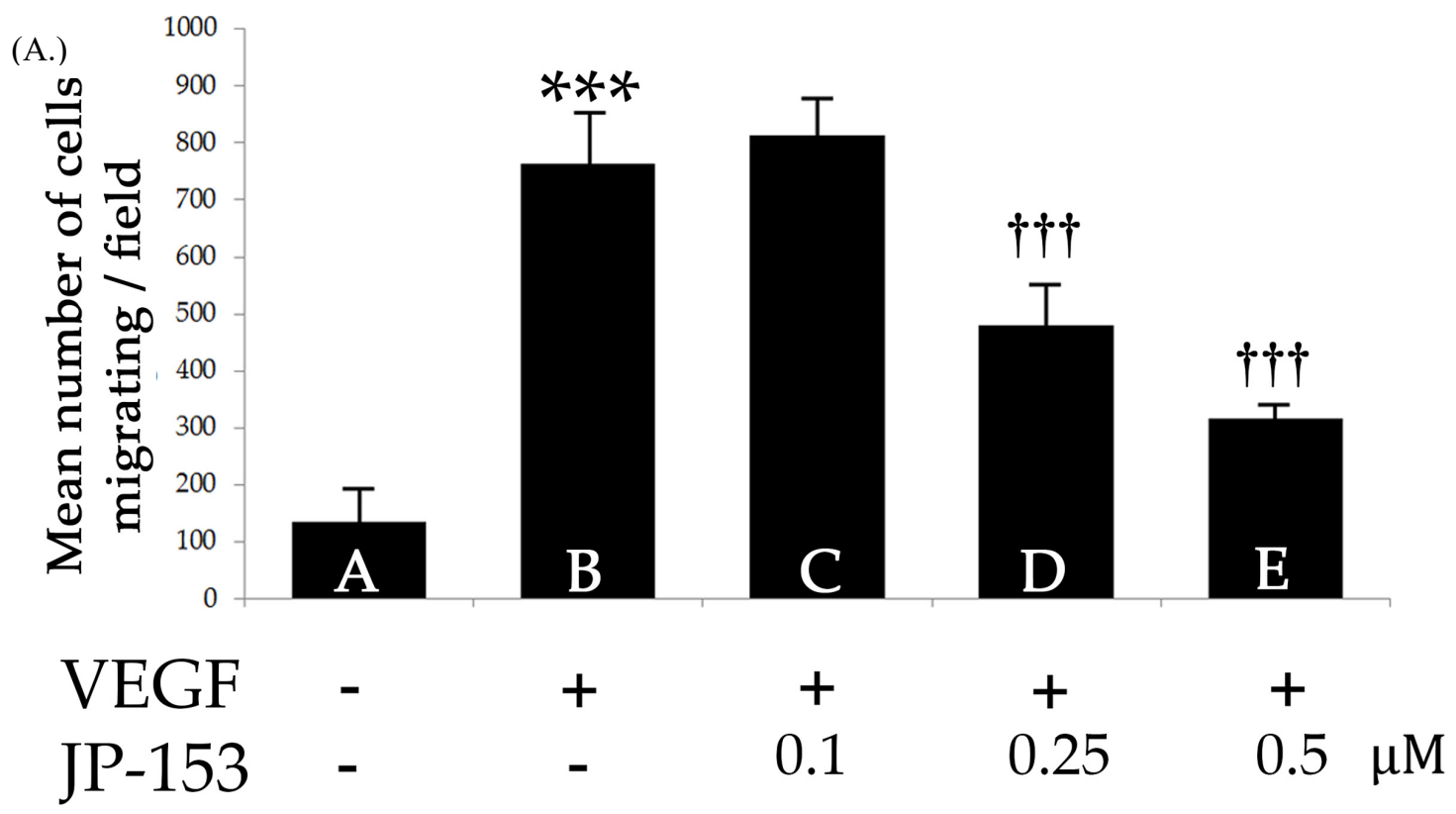

(B.)
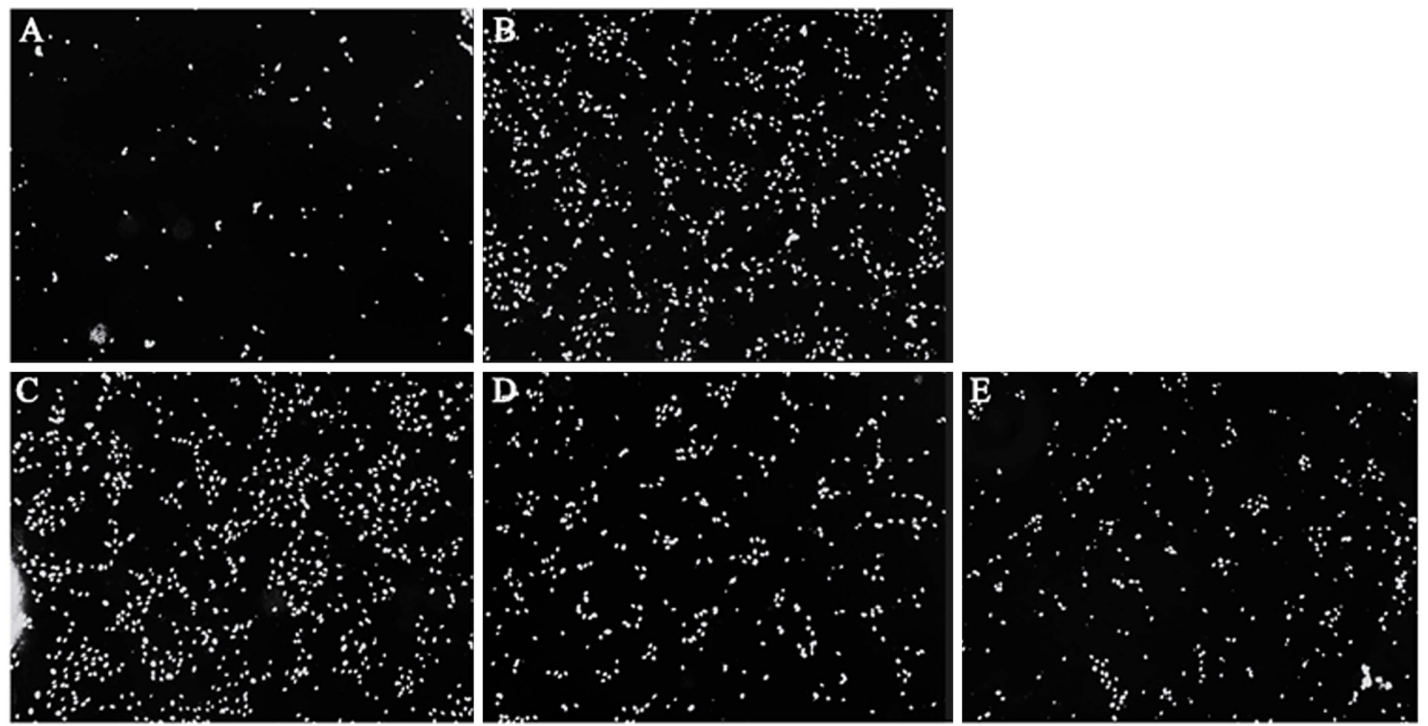

Figure 5-9. JP-153 inhibited VEGF-induced REC invasion.

RECs were seeded onto porous membranes and chemotactic factor, VEGF, was used to stimulate REC migration. Cells traversing the membrane were fixed and stained with DAPI, and representative images of each group are shown in panel $B$ (a-d; Serum-Free, VEGF, $\mathrm{V}+0.10, \mathrm{~V}+0.25, \mathrm{~V}+0.50 \mu \mathrm{M}$, respectively). Results show that JP-153 inhibited REC invasion in a concentration-dependent manner (data are mean $\pm \mathrm{SD}$; ***, $\dagger \dagger+\mathrm{P}<0.001 ; \mathrm{n}=6$ ). 


\section{FAT (FAK) \\ LD-2 : H1/4 H2/3 : LD-4}

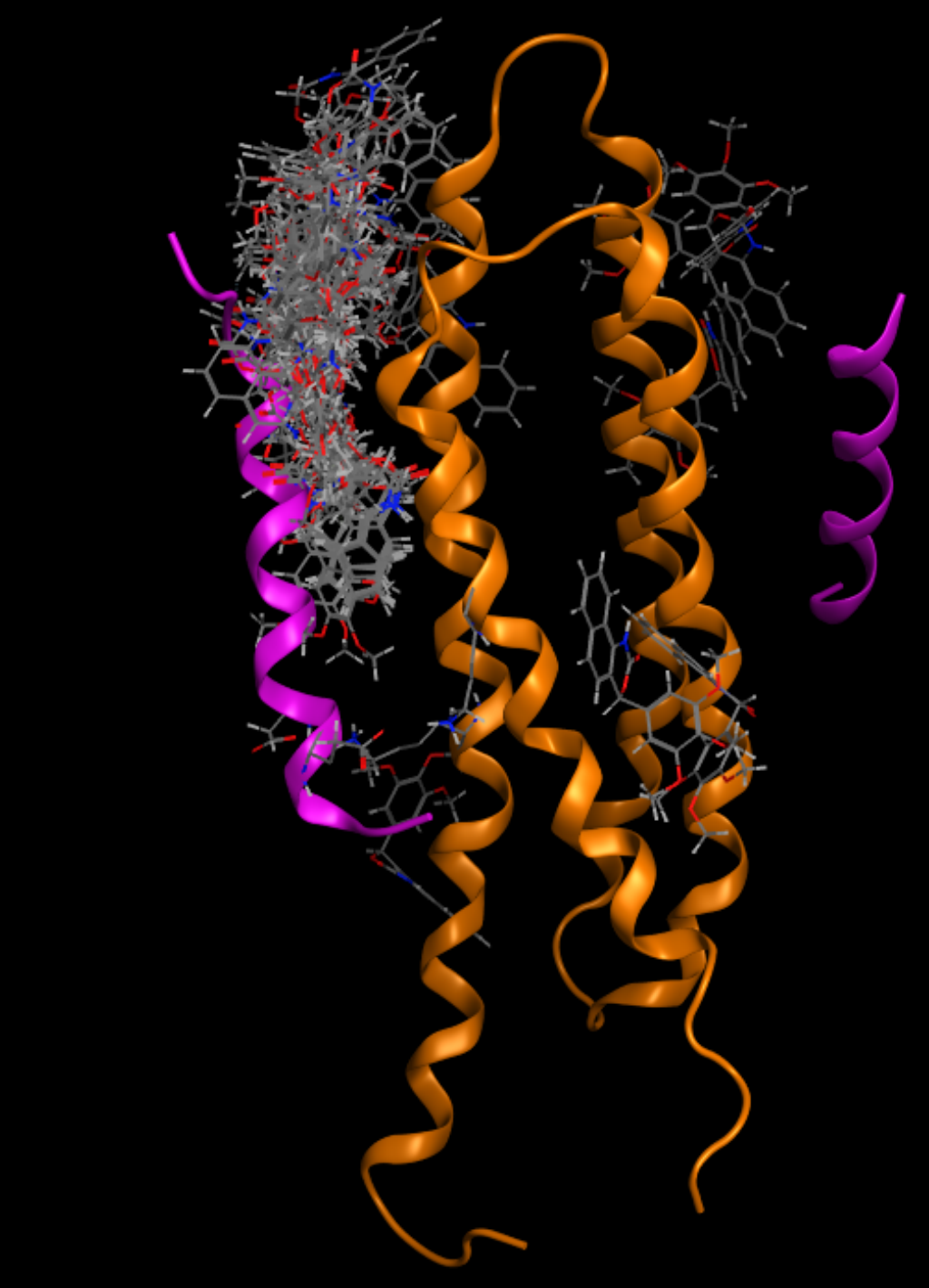

Figure 5-10. JP-153 in silico docking between the FAT domain and LD motifs.

3-D image of all interactions and poses of JP-153 were calculated using MOE. The majority of the orientations and poses of JP-153 (gray structures) indicated that JP-153 prefers the region between FAK's alpha-helices-H1/4 interface and LD-2 of paxillin. 
(A.)

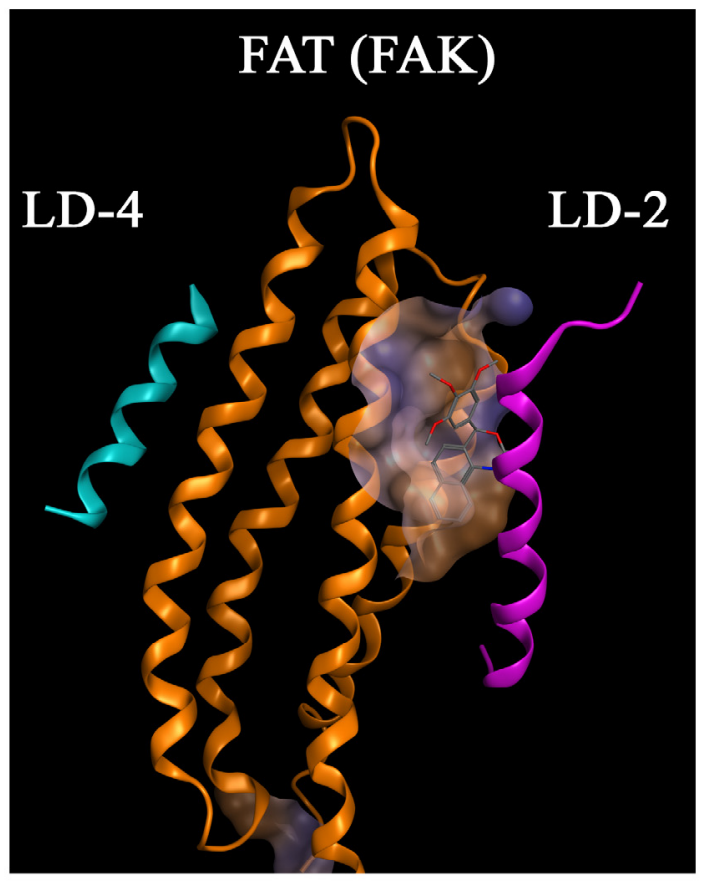

R-JP-153

(C.)

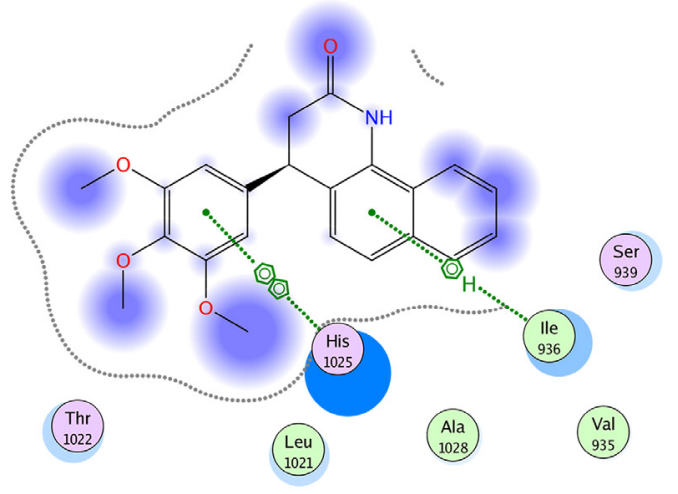

(B.)

\section{FAT (FAK)}

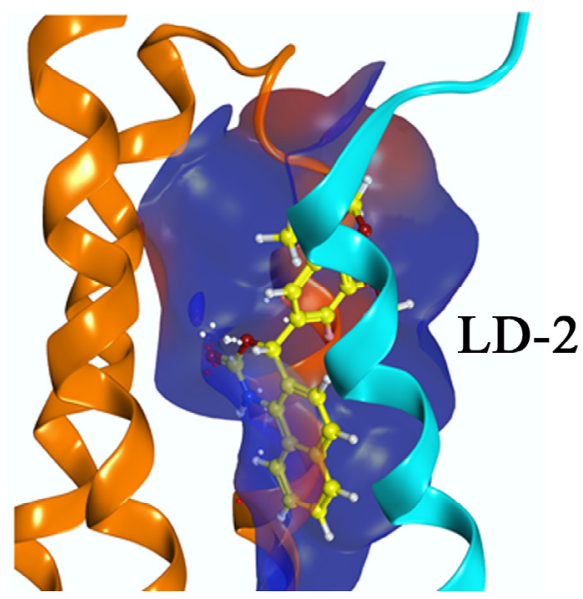

$$
\text { S-JP-153 }
$$

(D.)

$\left(\begin{array}{c}\text { Ala } \\ 1024\end{array}\right.$

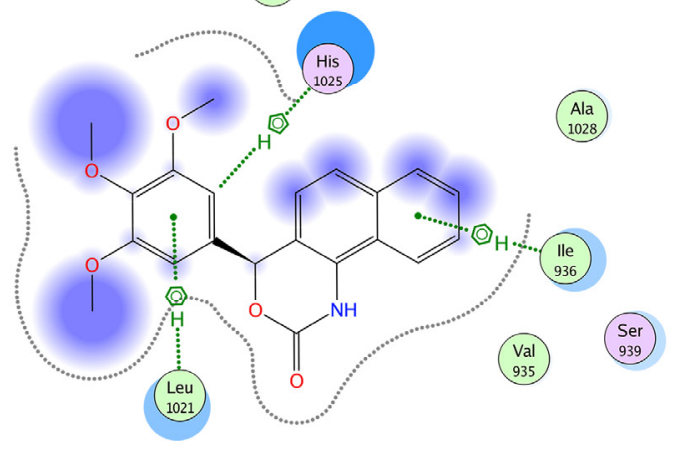

Thr
1022

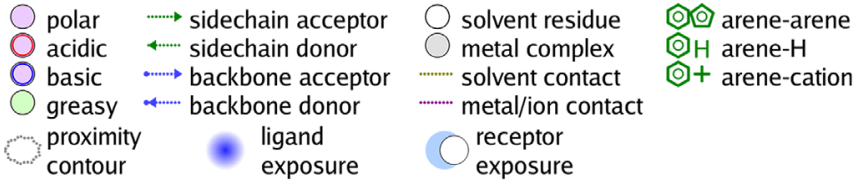

Figure 5-11. In silico interactions of JP-153 R and S enantiomers with FAT.

A-B) 3-D images of the R- and S- enantiomers of JP-153 positioned within the FAT domain's hydrophobic pocket bound to the LD-2 of paxillin. C-D) The corresponding ligand interaction profile of R-JP-153 (C) and S-JP-153 (D) show the amino acid residues Isoleucine 936, Histidine 1025, and Leucine 1021 (for the S-enantiomer potentially responsible for binding of JP-153 within the FAT domain. 


\section{JP-153 in vitro target engagement of the FAT domain inconclusive}

Based on the in silico docking calculations, JP-153 potentially targets the FAT domain as a potential modulator of the FAK-paxillin protein complex. Because paxillin binding partners are an abundant subset of proteins that can regulate angiogenesis, it was necessary to identify which protein(s) JP-153 binds. Thus, we developed a thermal shift assay to investigate the thermostability or change in thermal denaturation temperature $(\Delta \mathrm{Tm})$ of peptides with or without JP-153 (Figure 5-12). First, we examined the FAT domain alone to establish consistent melting temperatures that correspond to this precise peptide in solution (Figure 5-12A). Our results showed Tm of the FAT domain in the thermal shift assay remained consistent with increases in protein concentration with 0.5 $\mathrm{mg} / \mathrm{mL}$ being the optimal concentration (Table 5-3). Testing JP-153 over a range of high micromolar concentrations $(20-80 \mu \mathrm{M})$ there was no shift in Tm of the FAT domain (Figure 5-12B). The melting temperatures for each tested concentration of JP-153 with the FAT domain are listed in Table 5-4. We attempted to repeat these studies using the full-length paxillin protein, but we were unable to determine the Tm of paxillin due to the apparent instability of the protein in current buffer conditions. Future work in our lab will be to determine buffers or stabilizing conditions for the paxillin protein itself, and investigate the protein-protein interactions of paxillin and FAK, using LD2 and LD4 peptide sequences to determine the complex's Tm.

\section{Disruption of Src/FAK/paxillin complex by JP-153 in vivo inhibits RNV in the murine oxygen-induced retinopathy model}

Our in vitro mechanism of action studies in RECs suggested that JP-153 inhibited proliferation and migration by disrupting Src/FAK/paxillin signaling pathway. Therefore, we hypothesized that JP-153 could inhibit retinal angiogenesis in vivo by reducing $\mathrm{Src} / \mathrm{FAK} /$ paxillin activity in the eyes of mice. We used the murine OIR model of RNV to test JP-153 at low and high topical doses $(0.5$ and $5.0 \mathrm{mg} / \mathrm{kg})$ applied daily to each eye during the hypoxic period, P12-P17 (Figure 5-13). Our data shows that JP-153 inhibited neovascularization by 40 and $45 \%$ in a dose-dependent manner, compared to vehicle treated eyes $(* * * \mathrm{P}<0.001)$. However, only JP-153 at the higher dose enhanced the avascular area compared to vehicle $(* * * \mathrm{P}<0.001)$.

Mouse pups kept outside of the OIR chamber for the entire study were also dosed with JP-153 (5 mg/kg) under identical age-based dosing regimens to evaluate any impact on retinal vascular development from treatment of either vehicle alone or JP-153. There were no obvious differences between vehicle and JP-153-treated retinas in mice not exposed to the changes in oxygen tension during the OIR studies (Figure 5-14). These findings suggest that JP-153 can act to regulate pathological RNV without affecting normal retinal blood vessel growth, or vasculogenesis. 

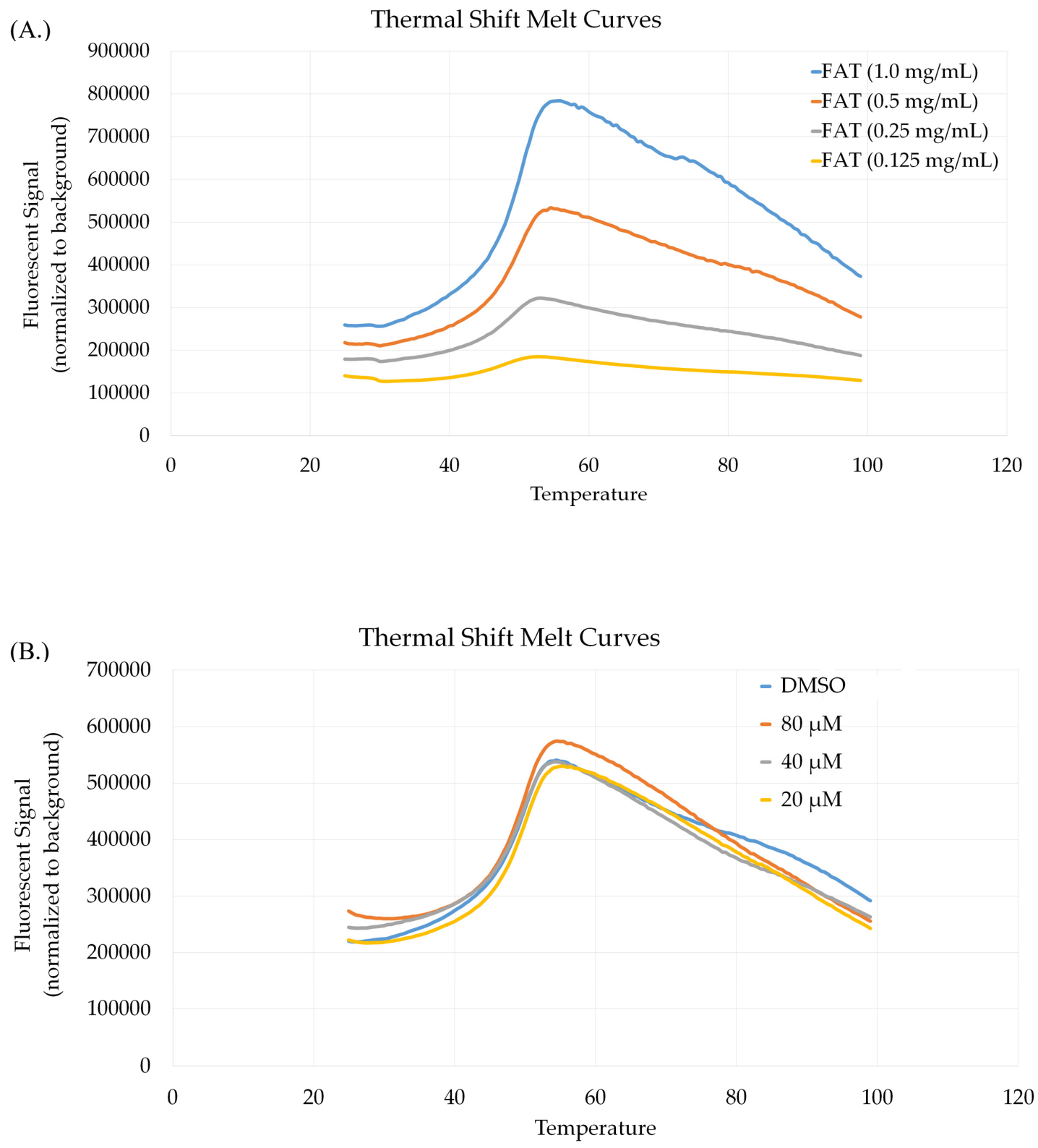

\section{Figure 5-12. Protein thermal shift assay of the FAT domain.}

A) Thermal shift plots for the focal adhesion targeting (FAT) domain of FAK in $0.1 \%$ CHAPS (110 mM NaCl, $50 \mathrm{mM}$ Tris HCl, 5 mM EDTA; $\mathrm{pH}$ 8) protein melt buffer. Thermostability of FAT was fairly consistent with increasing concentrations, by measure of melting temperature, Tm50 (see Table 5-3). B) Using FAT at $0.5 \mathrm{mg} / \mathrm{mL}, \mathrm{JP}-153 \mathrm{did}$ not affect the Tm or thermostability of the peptide (see Table 5-4) compared to DMSO $(0.1 \%)$. Experiments were performed in duplicate. 
Table 5-3. Melting temperatures (Tm) of increasing concentrations of FAT.

\begin{tabular}{ccc}
\hline FAT $[\mathbf{m g} / \mathbf{m L}]$ & $\mathbf{T m}$ & $\mathbf{\pm} \mathbf{S D}$ \\
\hline 1.000 & 52.75 & 0.07 \\
0.500 & 52.15 & 0.07 \\
0.250 & 51.22 & 0.05 \\
0.125 & 49.85 & 0.04 \\
\hline
\end{tabular}

Notes: $\mathrm{pH} \sim 8.0$; Tm: melting temperature as defined as the temperature at which half of the protein/peptide unfolds; SD: standard deviation. Vehicle used was $0.1 \%$ DMSO.

Table 5-4. Tm of the FAT peptide with increasing concentrations of JP-153.

\begin{tabular}{lccc}
\hline Peptide & JP-153 $[\boldsymbol{\mu M}]$ & Tm & 土 SD \\
\hline FAT & 0 & 52.15 & 0.06 \\
{$[\mathbf{0 . 5} \mathbf{~ m g / m L}]$} & 20 & 52.23 & 0.05 \\
& 40 & 51.89 & 0.05 \\
& 80 & 51.75 & 0.02 \\
\hline
\end{tabular}

Notes: $\mathrm{pH} \sim 8.0$; Tm: melting temperature as defined as the temperature at which half of the protein/peptide unfolds; SD: standard deviation. Vehicle used was $0.1 \%$ DMSO. 
(A.)

(B.)

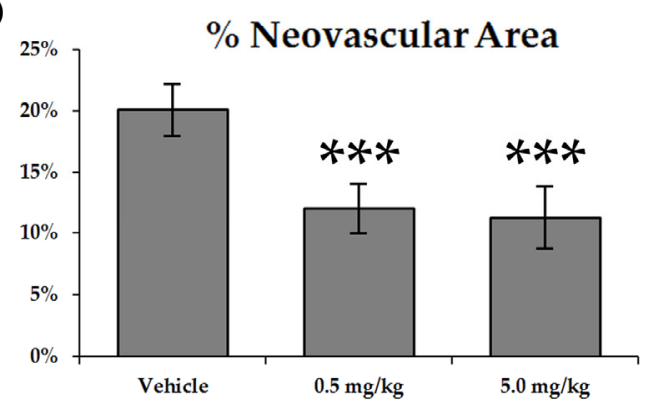

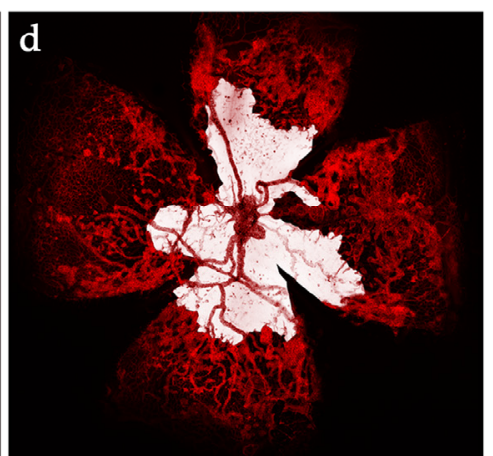

Vehicle

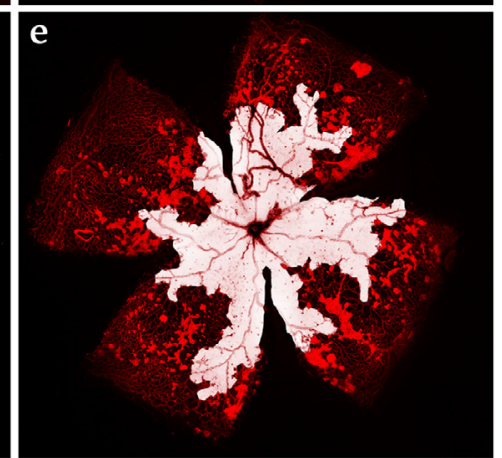

JP-153

$0.5 \mathrm{mg} / \mathrm{kg}$

JP-153

$5 \mathrm{mg} / \mathrm{kg}$
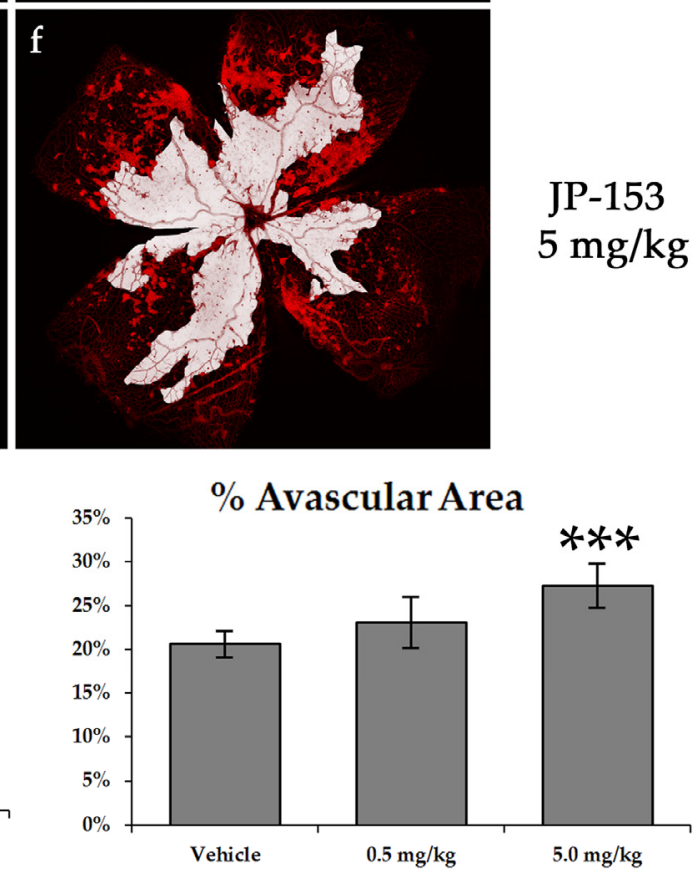

Figure 5-13. JP-153 inhibited retinal angiogenesis in the murine OIR model.

P17 retinal whole-mounts were stained for endothelial cells as described in Materials and Methods. A) Representative images of retina whole-mounts: neovascular area (a-c) and avascular area (d-f). B) Data analysis of retinal vasculature revealed that JP-153 inhibited $\mathrm{NV}$ and increased $\mathrm{AV}$ in a dose-dependent manner (data represent mean $\pm \mathrm{SD} ; * * * \mathrm{P}<$ $0.001 ; \mathrm{N}=8-14$ /group. 


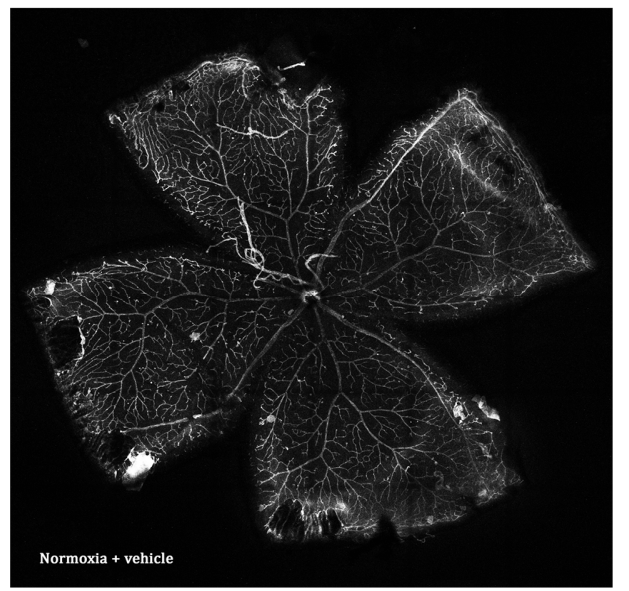

\section{N17 + Vehicle}

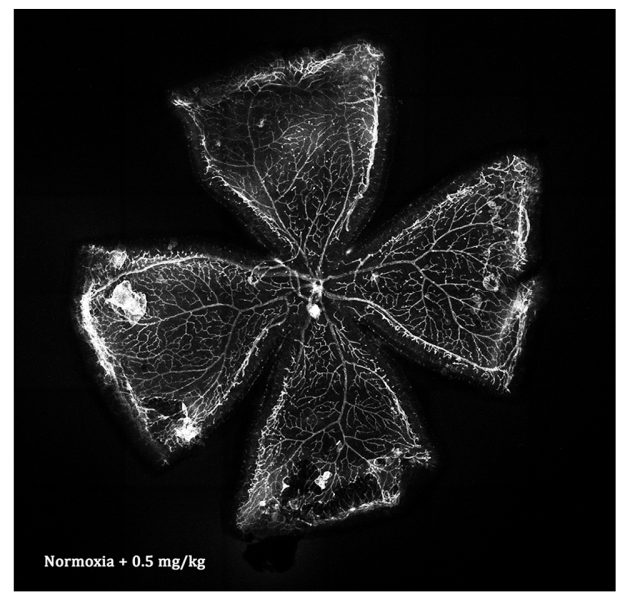

\section{$\mathrm{N} 17+0.5 \mathrm{mg} / \mathrm{kg}$ $\mathrm{JP}-153$}

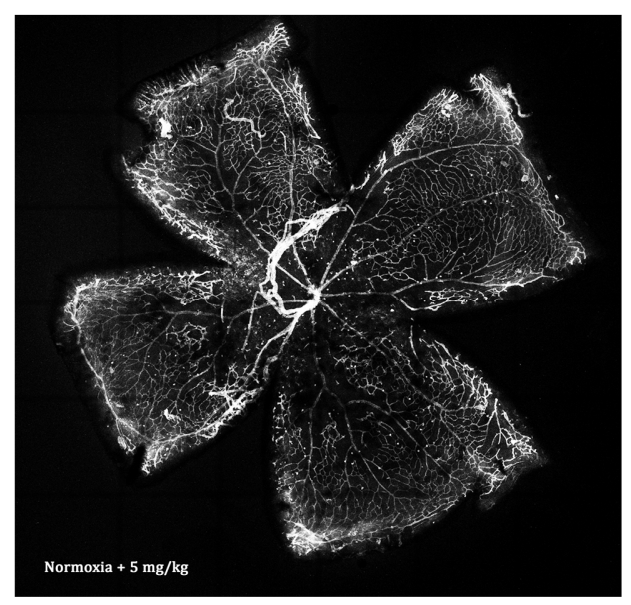

\section{$\mathrm{N} 17+5 \mathrm{mg} / \mathrm{kg}$ JP-153}

Figure 5-14. JP-153 does not affect vascularization in developing retinas.

Normoxia (N17) mice, subjected to room oxygen during experimental studies, were topically treated with 0.5 or $5 \mathrm{mg} / \mathrm{kg}$ daily during P12-P17. On P17, retinas were isolated, stained with Isolectin-B4, and imaged. There were no obvious signs of retinal vasculature disruptions or areas devoid of endothelial cells among JP-153 treated retinas. 


\section{Discussion}

In previous work, paxillin Y118 activation in high-dose radiation injury was an important signaling component driving REC proliferation in ischemic retinopathy [109]. We demonstrated herein that VEGF-dependent activation of the Src/FAK/paxillin signalsome is required for human retinal endothelial cell migration and proliferation. Moreover, we showed that disruption of the interaction between FAK and paxillin, using the small molecule JP-153, inhibited Src-dependent phosphorylation of the cytoskeletal protein paxillin (Y118) resulting in reduced migration and proliferation of RECs stimulated with VEGF; and that this effect did not interfere with VEGF-dependent activation of either Src or FAK. Preliminary docking studies identified a region between FAK's focal adhesion targeting (FAT) domain and the LD2 motif of paxillin where JP153 is likely to bind and thus, exert its antiangiogenic activity. Furthermore, topical application of a JP-153-loaded nanoemulsion inhibited hallmark features of pathologic retinal angiogenesis, neovascular tuft formation, and avascular area in the murine OIR model in a dose-dependent manner.

A major contribution of this study was that human primary RECs depend upon the Src/FAK activation of paxillin to direct VEGF-induced REC proliferation and migration, a signaling pathway well-characterized in cancer cells and other transformed cell lines but previously unknown in the primary human RECs [73, 218, 219]. We showed VEGF-dependent REC proliferation required Src-mediated activation of FAK and paxillin, recapitulating the numerous studies showing that $\mathrm{Src} / \mathrm{FAK}$ recruitment of paxillin drives endothelial cell proliferation [73, 220]. Our results correlated the activation of Y118 paxillin with enhanced proliferation in RECs.

Targeting paxillin with small molecules, we identified compound JP-153 as a potent inhibitor of REC proliferation. An unexpected and novel finding during in vitro mechanistic studies was that JP-153 targeted FA-mediated REC proliferation through decreases in paxillin Y118 levels. In agreement with our findings, others have postulated that decreasing activation of paxillin Y118 prevents the recruitment of downstream effector proteins of the FAC and thus disrupts FA signaling and inhibits cellular proliferation and migration $[219,221]$. Additional studies demonstrated that JP-153 did not inhibit the activation of the Src/FAK complex, per se, as there was no change to FAK phosphorylation. Additionally, we showed that JP-153 does not inhibit the kinase activity of either Src or FAK. These data strongly suggest that JP-153 acts to specifically disrupt paxillin protein interactions to inhibit downstream effector signaling, which may be complementary to the approach of targeting direct kinase activity, as seen with Src and FAK inhibitors currently in clinical trials. These results are supported by mutagenesis studies preventing FAK-paxillin binding within FAK's paxillin binding domains. FAKmutants did not show any effect on FAK activation, but did show inhibited paxillin binding and activation of downstream proteins [222, 223].

Activation of Src/FAK drives proliferation and migration through intermediates, ERK and Akt [224, 225]. Since JP-153 did not affect Src/FAK activation but did reduce paxillin Y118, we probed for these downstream markers to see whether JP-153 disrupts 
effector signaling. Our results showed that much like SU6656, JP-153 reduced Akt (Ser473) phosphorylation, but not ERK 1/2. We prevented Akt phosphorylation with LY294002, which significantly inhibited REC proliferation. LY294002 did not inhibit Src-mediated activation of FAK or paxillin. Since Akt is a known regulator of cellular migration, and is activated through the Src/FAK complex [226, 227], we tested and confirmed that JP-153 inhibited VEGF-induced migration by blocking the activation of paxillin and Akt. These results agreed with other studies that established the important role of the Src/FAK complex as crucial activators of the PI3K-Akt pathway, distinct from ERK-driven migration [228-230]. This data supported the role of Akt as an important downstream effector of FA complexes containing paxillin. The uncoupling of an active Src/FAK complex with paxillin and its downstream effectors suggested paxillin is a key regulator of pathologic FA signal transduction and potentially represents a novel in vivo mechanism and target, which would be distinct from anti-VEGF therapies aimed at silencing downstream receptor-mediated signaling.

Structural studies confirmed that the LD2 and LD4 motifs in paxillin form amphipathic $\alpha$-helices that interact with FAK's C-terminal FAT domain through two binding sites formed through four-helix $(\alpha-\mathrm{H} 1,-\mathrm{H} 2,-\mathrm{H} 3$, and $-\mathrm{H} 4)$ bundle conformations [216, 231-233]. Binding between paxillin and FAK is essential to the activation and localization of the FAC $[216,234]$ and pharmacological targeting of the FAT domain inhibited signaling mechanisms of growth-factor induced angiogenesis [235]. Thus, from these data and our extensive mechanistic work, we rationalized that the interaction between paxillin and FAK is where JP-153 acts to disrupt angiogenic signaling. We confirmed this in silico through docking calculations in MOE, which predicted JP-153 docks within the FAT domain hydrophobic interface, specifically the $\alpha-\mathrm{H} 1 / \mathrm{H} 4$ pocket and identified potential amino acid residue target sites comprising FAK residues Ile936, Leu1021 and His1025. Mutagenesis within FAT causes disruption of protein interactions between FAK and paxillin, lowered their phosphorylation (activation), and impaired the recruitment of FAK, paxillin and PI3K to FAs [82, 222, 226]. These findings support what we show in vitro with JP-153 in RECs, specifically with decreased paxillin Y118 phosphorylation and inhibition of p-Akt downstream. Intriguingly, the S-enantiomer of JP-153 showed a slightly enhanced (more negative) binding free energy compared to the R-enantiomer when docked inside the FAT domain. Potential reasons for this may be a result of two amino acids interacting with the tri-methoxy ring system, while the Renantiomer only possesses the ability to interact with one residue. These data suggest, albeit vaguely, that there may exist stereoisomer differences in potency of JP-153. Thus, these results are inherently limited due to our current molecular docking model, which lacks necessary intra-class distinguishability to assert differences in pharmacological effects based upon predicted binding strength. These studies require more testable analogs and extensive phenotypic data to better correlate model predictions. These studies are currently underway.

Thermal shift studies were performed to validate our in silico docking models of JP-153 bound to the four-helix bundled FAT domain. We first were able to establish a consistent Tm for the FAT domain over a range of concentrations using CHAPS buffer at $\mathrm{pH} \sim 8$. However, inhibition studies with JP-153 were not conclusive as data showed that 
there was no effect on Tm at any concentration (up to $80 \mu \mathrm{M}$ ). There are a few possibilities for our target engagement results; 1) sub-optimal buffer conditions ( $\mathrm{pH}$, ionic buffer strength, temperature, etc.) may have interfered with protein stability of FAT, or 2) JP-153 does not target the FAT domain for modulation of the focal adhesion complex. While this latter case is definitely a possibility, our in vitro mechanistic results, literature-backed activities underlying FA protein assembly and phosphorylation, and previous reports of 6-B345TTQ inhibiting FAT-paxillin interactions make this highly unlikely. However, the remaining pertinent question is how do specific buffer conditions change the thermostability or dynamics of the FAT domain? Both mechanical stability and thermal properties of the FAT peptide have been studied previously and these reports offered clues that buffer $\mathrm{pH}$ conditions are especially important for the orientation and nature of the amino acid residues within the four-helix bundle domain [236, 237]. Mechanical stability of FA proteins, both in isolation and in complex, are wellmaintained under force at relatively high and low $\mathrm{pH}$, with most mechanical instability arising from $\mathrm{pH}$ 6.5. However, these studies also concluded that the nature of FAs themselves are most highly $\mathrm{pH}$-dependent over a range of 5-8. However, with increasing $\mathrm{pH}$ during thermostability studies, the residues that align to create the two hydrophobic $\mathrm{H} 1 / 4$ and H2/3 interfaces of FAT become more exposed to solvent thus lowering the Tm [237]. In our hands, the FAT domain denatures around $50^{\circ} \mathrm{C}$, while in other assays, a $\mathrm{pH}$ of less than 7 increased the Tm by nearly $20^{\circ} \mathrm{C}$. Therefore, a protein melt buffer of $\mathrm{pH} 8$ may not be optimal for the thermal shift assay with FAT. Further refinement of these preliminary buffer conditions will be essential to our acceptance or rejection of our hypotheses stating that JP-153 targets the FAT domain to inhibit paxillin and FAK interactions.

Studies using targeted deletions of FA proteins, FAK and Src, in the mouse retina disrupt the progression of RNV [188, 238]; findings that correspond with our in vitro results using the Src inhibitor, SU6656, which affects all downstream binding and activation partners. We show similar in vitro effects with JP-153 on proliferation as with SU6656, specifically with decreased paxillin Y118 phosphorylation and inhibition of pAkt downstream, resulting in potent inhibition of movement and growth. From these studies, we can assert that the activation of the FAC may be a crucial component to the regulation of pathological retinal angiogenesis, in vivo. We tested this hypothesis by administering JP-153 topically in the OIR model, which resulted in significantly reduced retinal angiogenesis, as measured by both neovascularization and the avascular area. Intriguingly, we found that only the higher doses of JP-153 were able to significantly enhance avascular area, suggesting perhaps, that our small molecule affects pathologic neovascularization greater than vasculogenesis. Since VEGF can participate in both pathological and physiological growth, it is not surprising that anti-VEGF therapeutics can potently inhibit vascular growth and retinal function. These deficits were a result of significant structural changes to the retinal layers, despite having prevented the classic neovascular pathology [199]. These findings raise concerns as to whether or not enhancing the avascular area, or preventing revascularization with anti-VEGF treatment may exacerbate ischemic injury in neuroretinal tissues [239]. We used the same dosing regimen of JP-153 in mice reared in atmospheric conditions (room air) and found that even high-dose treatments did not affect normal vasculogenesis, as there were no obvious 
defects in "normal" vessel growth patterns. Our findings point to an important difference between anti-VEGF therapies and JP-153, with respect to dose effect on vasculogenesis, that suggests that JP-153 might help to avoid adverse effects associated with anti-VEGF monotherapy in patients long-term by sparing normal physiological homeostasis and neuroretinal function.

In conclusion, our results detail an effective strategy to treat pathological RNV using a small molecule drug. To our knowledge, this report is the first paradigm validating small molecule modulation of paxillin within FAs to prevent pathologic angiogenesis in neovascular disease. The fact that signaling through FA proteins underlie many aggressive diseases and are the therapeutic strategies in numerous pre-clinical and clinical studies, including cancer metastasis and polycystic kidney disease, creates a valid rationale for targeting the Src/FAK/paxillin signalsome [240-242]. Recently, small molecule kinase inhibitors of paxillin binding partners, Src and FAK, have advanced to late-stage clinical trials in humans, which suggests FA signal transduction can be effectively and safely modulated in humans [243, 244]. Paxillin, however, has never been successfully targeted by pharmacologic intervention for the treatment of any proliferative disease, even though its expression has been correlated with highly invasive cancers [245]. Moreover, the ability of paxillin to function as a scaffold protein that can bind multiple FA proteins represents a robust and opportunistic target to more effectively treat pathologic neovascularization. Since adaptive resistance is a major obstacle plaguing the efficacy of current anti-angiogenic treatments [52], the novelty of this current study can be characterized by two major findings: 1) paxillin is an important and viable target in pathologic retinal angiogenesis, and 2) JP-153 effectively modulates paxillin in vitro and in vivo to treat RNV (Figure 5-15). Thus, the target and mechanism of JP-153 has extensive applicability across a wide range of proliferative indications and warrants further pharmaceutical development and refinement as a novel therapeutic. 

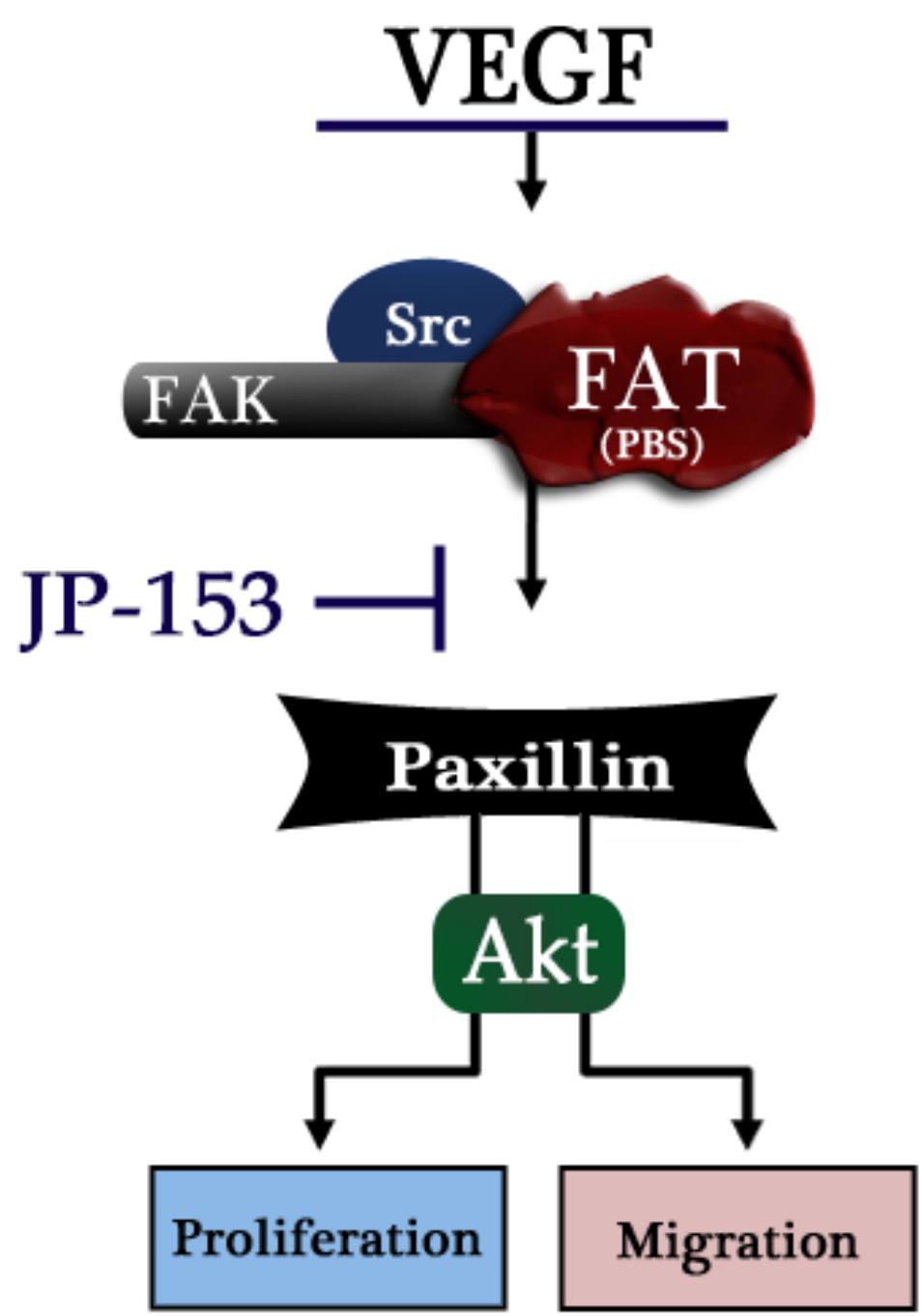

\section{Neovascularization}

Figure 5-15. Summary diagram depicting JP-153's proposed target of action.

Data suggests that JP-153 targets the interaction between an active Src/FAK signaling complex and paxillin. Inhibiting this interaction resulted in decreased paxillin activation (Y118) and preventing of downstream effector protein Akt activation. This effect translated into potent inhibition of REC proliferation and migration, in vivo, and inhibition of RNV, in vivo. 


\section{CHAPTER 6. PHARMACOKINETICS OF JP-153}

\section{Introduction}

Age-related macular degeneration (AMD) and diabetic retinopathy (DR) accounts for nearly 4 million blind people worldwide [246]. Diagnoses of AMD and DR have increased due, in large part, to the world's aging and/or obese populations, as diabetes is among the most common co-morbidity among patients in the developed world. These 371 million diabetics (expected to reach half-a-billion by 2030) exemplify an urgent and imperative need for the discovery and development of novel AMD and DR therapeutics to prevent blindness [247, 248].

DR and AMD are chronic degenerative diseases associated with pathological angiogenesis and retinal hemorrhaging in the eye. AMD and DR are characterized by an enhanced expression of vascular endothelial growth factor (VEGF) which promotes retinal and choroidal neovascular outgrowths of highly fenestrated endothelial cells during PDR and AMD, respectively [33, 34, 195, 249-251]. Thus, we have validated the role of paxillin in focal adhesion-dependent signal transduction under both in vitro and in vivo models of retinal angiogenesis. JP-153 effectively modulated FA-dependent growth factor signaling to suggest that targeting paxillin is a viable option for the treatment of pathologic neovascularization in both wet AMD and proliferative DR.

In this chapter, we characterize the in vitro and in vivo pharmacokinetics of compound JP-153 (Figure 6-1). We evaluated the systemic and oral pharmacokinetics using absorption, distribution, metabolism, elimination (ADME) as study endpoints. We measured JP-153 plasma concentrations both intravenously and after oral administration to establish and define the PK characteristics of JP-153 in Lewis rats.

\section{Materials and Methods}

\section{Reagents}

JP-153 and internal standard (IS) BA-126 were synthesized and verified to be $>96 \%$ pure by NMR spectroscopy. All mobile phase solvents and water of HPLC grade were purchased from Fisher-Scientific (Pittsburgh, PA, USA). Lithium heparinized drugfree Lewis rat plasma was obtained from BioreclamationIVT (Hicksville, NY).

\section{LC-MS/MS parameters}

The LC-MS/MS system comprised an API 4500 Q TRAP mass spectrometer equipped with an electrospray ion source (Applied Biosystems Sciex, Foster City, CA) and operated in negative-ion mode was used for MS detection. Quantitation was 
JP-153, MW : 365 g/mol

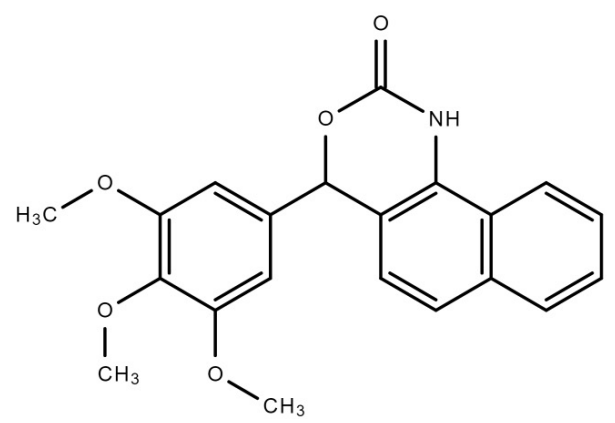

IS (BA-126), $\mathrm{MW}: 379 \mathrm{~g} / \mathrm{mol}$

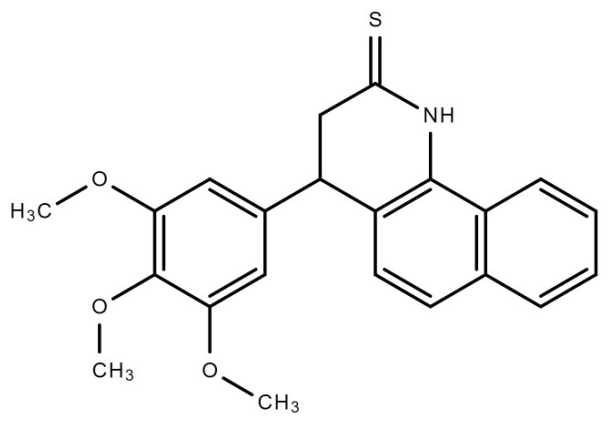

Figure 6-1. Structures of JP-153 and internal standard (IS), BA-126. 
performed using multiple reaction monitoring (MRM) mode to study parent $\rightarrow$ product ion transitions for JP-153 $(368.85 \rightarrow 197.70)$ and IS $(379.57 \rightarrow 211.70)$ with unit resolution. Source dependent parameters optimized were gas 1 (nebulizer gas): 40 psi, gas 2 (heater gas): $40 \mathrm{psi}$, ion spray voltage (ISV): $-5500 \mathrm{~V}$, temperature: $500^{\circ} \mathrm{C}$. JP- 153 and IS dependent parameters were declustering potential $(-50 \mathrm{~V})$, entrance potential $(-10$ $\mathrm{V})$, collision energy $(-23 \mathrm{~V})$, and collision cell exit potential $(-16 \mathrm{~V})$ were the same for both JP-153 and IS. Nitrogen was used as collision-activated dissociation (CAD) gas and was set at 7. Quadrupole 1 and quadrupole 3 were maintained at unit resolution and dwell time was set at $250 \mathrm{~ms}$ for each MRM transition. Chromatographic separation was performed on a Waters reverse-phase Symmetry C8 column $(5 \mu \mathrm{m}, 100 \times 2.1 \mathrm{~mm}, 100 \AA$; Waters Corp. Milford, MA) held at $25^{\circ} \mathrm{C}$. The mobile phase consisted of eluent A (water) and eluent $\mathrm{B}$ (methanol), and separation achieved using a gradient program of 0.5 min: $20 \% \mathrm{~B}$; $1.4 \mathrm{~min}: 90 \% \mathrm{~B} ; 3.5 \mathrm{~min} 90 \% \mathrm{~B} ; 4.0 \mathrm{~min}: 20 \% \mathrm{~B}$, at a flow rate of 0.4 $\mathrm{mL} / \mathrm{min}$ performed with a run time totaling $5.0 \mathrm{~min}$. Analytical data were processed using the software program Analyst (Version 1.6.1).

\section{LC-MS/MS method validation}

Calibration curves were constructed by plotting the peak area ratio of JP-153: IS (BA-126) against the nominal concentration of calibration standards. For each batch of samples, a calibration curve was constructed from the results of duplicate measurement of 12 concentrations of JP-153 (serially diluted over a doubling range of $0.488-1000$ $\mathrm{ng} / \mathrm{mL}$ ). The peak area ratios of JP-153 to IS versus corresponding concentrations were used for the linear least-squares regression of the calibration lines and for determination of slopes $(A)$, intercepts $(B)$, and correlation coefficients. Unknown sample concentrations of JP-153 were calculated from the quadratic regression equation with a weighted factor of $1 / x$. The lower limit of quantification (LLOQ) was defined as the lowest concentration with a signal to noise $>5$ over three independent runs with coefficient of variation $\%$ accuracy within $20 \%$ and precision within $20 \%$ for every run. The limit of detection (LOD) was not determined. Three quality control (QC) validation samples were prepared at $1.95,15.63,125 \mathrm{ng} / \mathrm{mL}$ in rat plasma. All chromatograms were processed using the automatic integration software module in Analyst 1.6.1 followed by manual check to confirm proper integration of each peak area.

Matrix effect and recovery were evaluated according to published literature [252]. Briefly, the matrix effect was determined by comparing the analytical response of the standards spiked into plasma extracts (Solution B) with that of the neat standard solutions (Solution A). The loss of signal represents the ion suppression. The extraction recovery of JP-153 from rat plasma was determined by comparing the analytical response of processed quality control samples (Solution C) with that of blank plasma extracts spiked with standard working solutions (Solution B). They were expressed as: matrix effect (\%) $=(\mathrm{B} / \mathrm{A}) * 100$; recovery rate $(\%)=(\mathrm{C} / \mathrm{B}) * 100$. These experiments were performed in triplicate at concentration level 1.95, 15.63, and $125.0 \mathrm{ng} / \mathrm{mL}$. 
Method selectivity was evaluated by comparing the chromatograms of six blank plasma samples from six different untreated rats for interference at the retention times of JP-153 and IS. The intra- and inter-day precision and accuracy were estimated by parallel analytical runs performed on the same day or on two different days. Each analytical run consisted of a matrix blank, a set of calibration standards, six replicate LLOQ samples, and a set of three levels of QC samples. The acceptance criteria of the data included precision within $15 \%$ relative standard deviation and accuracy within $\pm 15 \%$ deviation of the nominal value. Ion suppression via matrix effect was evaluated by adding three level

JP-153 QC sample with and without IS to extracted blank plasma comparing JP-153 peak areas to neat solution samples. Post-preparative autosampler stability $\left(+4^{\circ} \mathrm{C}\right)$ was determined by re-injection of samples $24 \mathrm{~h}$ following initial injection.

\section{Sample preparation}

Protein precipitation was used to extract JP-153 from rat plasma. An aliquot $(50 \mu \mathrm{L})$ was added in precipitation solution $(100 \%$ Acetonitrile), vortexed for $30 \mathrm{~s}$, and then centrifuged for $15 \mathrm{~min}$ at $4{ }^{\circ} \mathrm{C}(12,000 \times g)$. The supernatants were transferred to analytical vials and an aliquot $(5 \mu \mathrm{L})$ was injected onto the $\mathrm{LC}-\mathrm{MS} / \mathrm{MS}$ system.

\section{Pharmacokinetic study design}

Pharmacokinetic studies were performed as previously described [166, 167, 253, 254]. Animals will receive either a single intravenous or oral dose of JP-153 (5, 10 $\mathrm{mg} / \mathrm{kg}$, respectively). Briefly, pre-catheterized male Lewis (LEW/Crl) rats were purchased from the Charles River Laboratory (Wilmington, MA). Following intravenous administration, blood samples were withdrawn from the jugular vein cannula at regular intervals after dosing $(5,15,30$ minutes and $1,4,8,12$, and 24 hours) and the plasma were extracted and stored at $-80^{\circ} \mathrm{C}$ until analysis. After oral administration, blood was sampled at regular time intervals (15, 30 minutes and 1, 4, 8, 12, and 24 hours) and plasma was extracted and samples were stored at $-80^{\circ} \mathrm{C}$ until analysis. JP-153 concentrations were analyzed using the LC/MS/MS based on the methodology described previously [254]. JP-153 plasma concentrations from IV and PO routes of administration were analyzed using standard non-compartmental analyses in WinNonlin (Ver. 7.0, Pharsight, Mountain View, CA).

\section{Pharmacokinetic data analysis}

The concentration-time profiles for both PO and IV administration were analyzed by non-compartmental analysis in WinNonlin (Pharsight). The area under curve (AUCinf) from time zero to infinity was calculated using the trapezoidal rule by extrapolating to time infinity. The systemic clearance (CL) was calculated using the equation: 


$$
C L=\frac{\operatorname{Dose}_{i v}}{A U C_{i n f, i v}}
$$

where Dose $_{i v}$ and $\mathrm{AUC}_{\mathrm{inf}, \mathrm{iv}}$ are the IV dose and area under the plasma concentration-time curve from time 0 to infinity, respectively. Oral bioavailability (F) was calculated using

$$
F=\frac{A U C_{i n f, \text { oral }} * \text { Dose }_{\text {iv }}}{A U C_{\text {inf }, \text { iv }} * \text { Dose }_{\text {oral }}}
$$

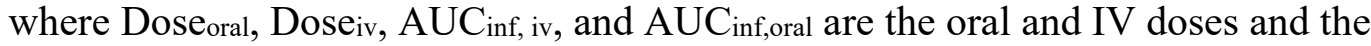
corresponding areas under the plasma concentration-time curves from time zero to infinity, respectively.

\section{Microsomal stability of JP-153}

JP-153 $(1 \mu \mathrm{M})$ was assessed in pooled liver microsomes from human, mouse, and rats [166]. Briefly, JP-153 was incubated in duplicate with microsomes at $37^{\circ} \mathrm{C}$. Reactions contained microsomal protein in $100 \mathrm{mM}$ potassium phosphate, $2 \mathrm{mM}$ $\mathrm{NADPH}, 3 \mathrm{mM} \mathrm{MgCl} 2, \mathrm{pH}$ 7.4. NADPH was omitted to detect NADPH-free degradation. At $60 \mathrm{~min}$, the reaction was stopped by adding ice-cold methanol (+ internal standard). Samples were centrifuged to remove precipitated protein, and the supernatants were analyzed by $\mathrm{LC} / \mathrm{MS} / \mathrm{MS}$ to quantitate the remaining parent.

\section{P-450 interaction studies}

CYP-450 interaction studies were conducted in pooled human liver microsomes. CYP-450 inhibition (\%) by JP-153 $(1 \mu \mathrm{M})$ was compared to control inhibitors $(1 \mu \mathrm{M})$ for the most common CYP-450 isoforms, 3A4, 2C9, 2D6, 2C19, and 1A2.

\section{In vitro off-target screen}

Counter-screen assays to determine "off-target" effects are conducted at an early stage to mitigate toxicological risk of new chemical entities[255]. Thus, counter-screen assays to determine "off-target" effects were conducted to assess potential toxicological risk of JP-153. At $10 \mu \mathrm{M}$, JP-153 was screened against a wide variety of target receptors, enzymes, and ion channels using a serum-free cell-based ExpresS Profile assay (Eurofins, CEREP; Redmond, VA). Interactions greater than 50\% were considered a "positive" interaction and further tests were conducted to distinguish agonist or antagonist activity. 


\section{Results and Discussion}

\section{JP-153 method validation}

Assay selectivity was assessed at the LLOQ using six drug-free samples from six different plasma lots. No interfering peaks at the retention times of either JP-153 or IS were observed in blank extracted plasma. Additionally, no chromatographic peaks corresponding to the retentions times for either JP-153 or IS were detected in pre-dose rat plasma. Figure 6-2 displays representative chromatograms of blank plasma and plasma spiked with JP-153 and IS.

Calibration curves for JP-153 were quadratic over the concentration range of $0.488-1000 \mathrm{ng} / \mathrm{mL}$ for plasma samples. The correlation coefficient $\left(R^{2}\right)$ for all assays was greater than 0.999 with weighting (1/x). The LLOQ for JP-153 was $1.95 \mathrm{ng} / \mathrm{mL}$ for plasma, with an accuracy of $117 \pm 1.4 \%(\mathrm{CV}=1.21 \%)$ and within $98.2 \pm 3.5 \%(\mathrm{CV}=$ $3.56 \%$ ) of other concentrations levels. The range of inter- and intra-day accuracy and precision values in plasma were $0.22-15.6 \%$ and $1.21-14.1 \%$, respectively. Absolute recovery of JP-153 from plasma ranged from 88-93\% using low, mid and high QC samples (1.95-125 ng/mL) (Table 6-1). The mean extraction recovery for the IS (10 $\mathrm{ng} / \mathrm{mL}$ ) was $86.5 \pm 6.9 \%$. The matrix effect of JP-153 in plasma was $102.4 \pm 16.3 \%$. Degradation of samples in the autosampler at $+4^{\circ} \mathrm{C}$ over a period of 24 hours was determined $<10 \%$ for both JP-153 and IS.

\section{Intravenous and oral in vivo pharmacokinetics of JP-153 in Lewis rats}

The above validated LC-MS/MS method was used to study the systemic exposure of JP-153 in rats after either IV or oral administration. Figure 6-3 shows the concentration-time profiles of the average ( $n=6$ subjects/time point) plasma concentrations over 24 hours for JP-153 following intravenous and oral administration. Table 6-2 lists the pharmacokinetic parameters calculated from standard NCA in WinNonlin. JP-153 was found to have a rapid clearance $(2.19 \pm 0.35 \mathrm{~L} / \mathrm{h} / \mathrm{kg})$, high volume of distribution $(\mathrm{Vd})(2.83 \pm 0.67 \mathrm{~L} / \mathrm{kg})$ and a terminal half-life of $0.887 \pm 0.12 \mathrm{hr}$. A comparison of the plasma exposure $\left(\mathrm{AUC}_{0 \rightarrow 24 \mathrm{~h}}\right)$ following a single intravenous and oral dose, revealed that oral bioavailbity $(\mathrm{F})$ could be estimated as $27.5 \%$. JP-153 extrapolated area under the curve (AUC) represented less than $15 \%( \pm 2.8)$ of AUC from time zero to infinity.

\section{In vitro ADME and off-target screening for JP-153}

Extensive metabolism of JP-153 occurred in the presence of NADPH with liver microsomes (Table 6-3). These studies revealed that JP-153 is largely metabolized in rodents with a mere $3 \%$ parent compound remaining in mice and just $0.3 \%$ in rats, after 60 minutes. However, percent JP-153 remaining is slightly higher in humans with an 


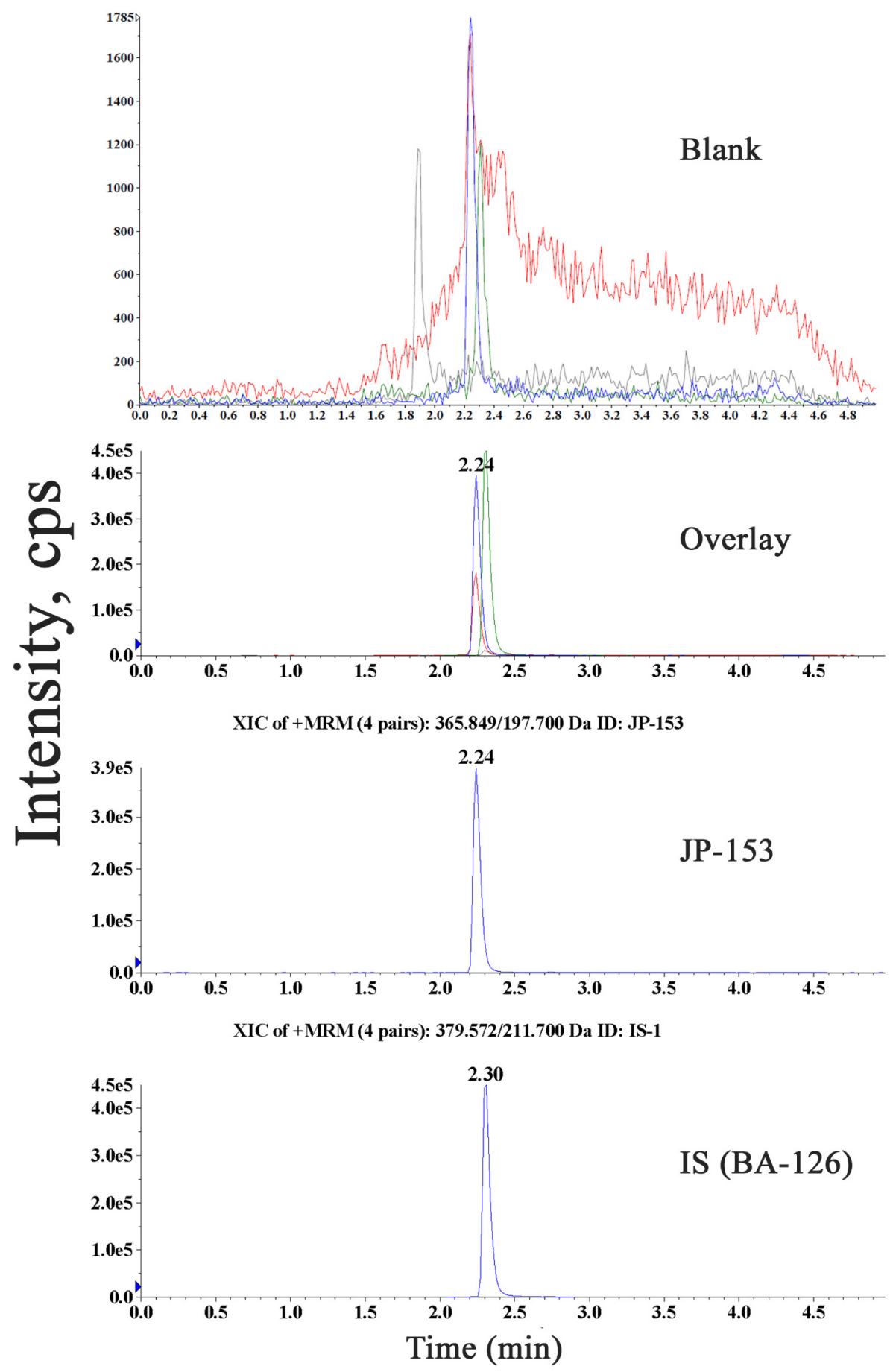

Figure 6-2. Chromatograms of blank plasma and JP-153/IS spiked plasma.

Extracted ion chromatograms (XIC) and retention times for JP-153 and BA-126 were 2.24 and 2.31 minutes with mass transitions monitored at 197.7 (365.85) and 211.7 (379.57) for JP-153 and IS, respectively. 
Table 6-1. JP-153 absolute recovery.

\begin{tabular}{lccc}
\hline $\begin{array}{l}\text { Conc. } \\
(\mathbf{n g} / \mathbf{m L})\end{array}$ & Recovery \% & \pm SD & CV\% \\
\hline 1.95 & 88.2 & 0.15 & 0.17 \\
15.6 & 86.4 & 1.53 & 1.78 \\
125 & 92.6 & 12.0 & 13.0 \\
\hline
\end{tabular}


(A.)
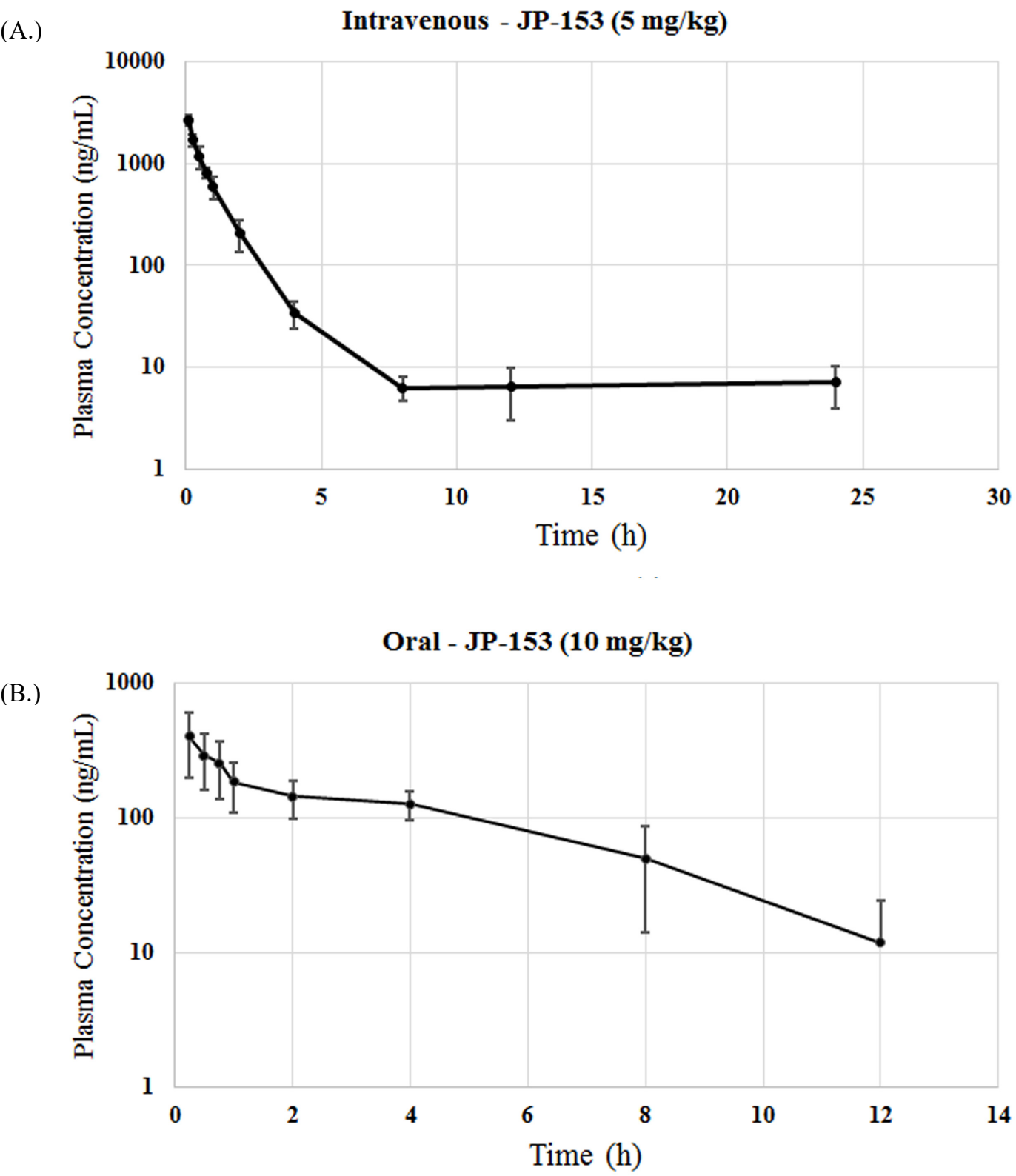

Figure 6-3. Average plasma concentrations of JP-153 in Lewis (LEW/Crl) rats.

A) Concentration-time profile of plasma concentrations after intravenous dose of JP-153 $(5 \mathrm{mg} / \mathrm{kg})(\mathrm{n}=6$ subjects/time point. B) Concentration-time profile of plasma concentrations after oral dose of JP-153 (10 mg/kg; $n=6$ subjects/time point). The 24 hour timepoint from oral data fell below the lower limit of quantification (LLOQ) and was removed from data used to generate the concentration-time plots. 
Table 6-2. Pharmacokinetic parameters of JP-153 after IV administration.

\begin{tabular}{lccccccrr}
\hline Compound & Route & $\begin{array}{c}\text { Dose } \\
{[\mathbf{m g} / \mathbf{k g}]}\end{array}$ & $\begin{array}{c}\mathbf{C}_{\mathbf{m a x}} \\
{[\mathbf{m g} / \mathbf{L}]}\end{array}$ & $\begin{array}{c}\mathbf{t}_{1 / 2} \\
{[\mathbf{h}]}\end{array}$ & $\begin{array}{c}\mathbf{A U C} \\
{[\mathbf{h} * \mathbf{m g} / \mathbf{L}]}\end{array}$ & $\begin{array}{c}\mathbf{C L} \\
{[\mathbf{L} / \mathbf{h} / \mathbf{k g}]}\end{array}$ & $\begin{array}{c}\text { Vd } \\
{[\mathbf{L} / \mathbf{k g}]}\end{array}$ & $\begin{array}{c}\text { F \% } \\
\text { (PO) }\end{array}$ \\
\hline JP-153 & IV & 5.0 & 2.67 & 0.887 & 2.29 & 2.19 & 2.83 & 27.5 \\
& & & \pm 0.31 & \pm 0.12 & \pm 0.34 & \pm 0.35 & \pm 0.67 & \\
\hline
\end{tabular}

Notes: Lewis ( $\mathrm{LEW} / \mathrm{Crl})$ rats were used for in vivo studies; $\mathrm{C}_{\max }$ : maximum concentration after the administration of dose; $\mathrm{t}_{1 / 2}$ : half-life; AUC: area under the curve (a measure of drug exposure); CL: clearance; Vd: volume of distribution; \% F: Percent bioavailability, calculated using the AUC values obtained after oral administration at $10 \mathrm{mg} / \mathrm{kg}$.

Table 6-3. JP-153 in vitro liver microsomal studies in human, mouse, and rat.

\begin{tabular}{|c|c|c|c|c|c|c|}
\hline \multirow{2}{*}{ Species } & \multirow{2}{*}{ Conc. } & \multicolumn{5}{|c|}{ \% Parent remaining } \\
\hline & & NADPH & $\mathbf{1}^{\text {st }}$ & $2^{\text {nd }}$ & Mean & \pm SD \\
\hline \multirow[t]{2}{*}{ Human } & $1 \mu \mathrm{M}$ & Yes & 37.5 & 41.8 & 39.7 & \pm 3.0 \\
\hline & & No & 106.9 & 101.4 & 103.7 & \pm 3.9 \\
\hline \multirow[t]{2}{*}{ Mouse } & $1 \mu \mathrm{M}$ & Yes & 2.8 & 2.5 & 2.7 & \pm 0.2 \\
\hline & & No & 99.5 & 99.6 & 99.6 & \pm 0.1 \\
\hline \multirow[t]{2}{*}{ Rat } & $1 \mu \mathrm{M}$ & Yes & 0.3 & 0.1 & 0.2 & \pm 0.1 \\
\hline & & No & 102.6 & 116.1 & 109.4 & \pm 9.5 \\
\hline
\end{tabular}

Notes: NADPH: nicotinamide adenine dinucleotide phosphate; SD: standard deviation. 
average of 39\% remaining after incubation with liver microsomes. Moreover, JP-153 lacks significant inhibition of the most clinically relevant P-450 enzyme family members 3A4 and 2D6 (Table 6-4) indicating JP-153 may in fact be substrates but its ability to inhibit these metabolic enzymes is nonexistent. In our previous in vivo studies, we showed significant improvements in hallmark features of an ischemic and neovascular retinas when JP-153 was treated topically to the eyes of mice. The fact that the compound is rapidly metabolized in vitro, gives us reason to suspect that once out of the eye, JP-153 may not reside in the circulatory system for very long, lowering the risk any systemic toxicities. This is key to ocular drugs including anti-VEGF protein therapeutics, as some early generation therapies are known to have deleterious effects on the cardiovascular systems [256, 257].

Information regarding inhibition and/or metabolism of a platform technology by major cytochrome P-450 enzymes can be useful. It identifies new compounds as potential drug-drug interaction (DDI) "victim" or "perpetrator". Additionally, any interaction with P-450 enzymes may imply rapid systemic clearance and/or low oral bioavailability making it difficult to achieve sustained systemic drug levels without frequent high doses. By identifying "metabolic hotspots", modifications can be made to alter platform stability [258]. Thus, since JP-153 is rapidly metabolized, further studies investigating the metabolites and identifying which subset of CYP enzymes are responsible will be key to the generation of future JP-153 analogs, especially if the mechanisms of action we posited in early chapters can be useful in the setting of other hyper-proliferative diseases, such as cancer.

Between 1992-2002, $33 \%$ of Phase III drug failures were attributed to an unacceptable side effect profile [259]. Thus, evaluation of adverse event potential is critical for early stage drug discovery and development [255]. In our experience, the following battery of complementary in vitro assays is typically requested: 1) genotoxicity (Ames, bacterial cytotoxicity, and in vitro micronucleus assays), 2) cardiac toxicity (hERG), and 3) off-target effects using in vitro pharmacological assays covering a broad range of targets including receptors, ion channels, transporters, enzymes and second messengers. JP-153 counter screening was conducted against a wide variety of target receptors, enzymes, and ion channels in a serum-free cell-based assay, ExpresS Profile (Eurofins). With significant interactions set at greater than 50\%, JP-153 was confirmed to interact with two surface receptors: serotonin receptor 5- $\mathrm{HT}_{2 \mathrm{~B}}$ and melatonin-1 receptor $\left(\mathrm{MT}_{1}, \mathrm{ML}_{1 \mathrm{~A}}\right)$ (Table 6-5). JP-153 at eight concentrations was evaluated for agonist or antagonist activity for both the 5- $\mathrm{HT}_{2 \mathrm{~B}}$ and $\mathrm{MT}_{1}$ receptors. No determinable interaction, agonist or antagonist, was evident for serotonin receptor 5-HT2B. However, JP-153 interacted with and activated the $\mathrm{MT}_{1}$ receptor with an $\mathrm{EC}_{50} \approx 45 \mu \mathrm{M}$ (Figure 6-4). This is not at all surprising considering the structural similarities of JP-153 with MT1 receptor agonists, specifically agomelatine [260]. In fact, during routine observations throughout OIR studies (Chapter 5), JP-153-treated mice were not as active as control groups (observational data). The activation of MT1 by endogenous agonist melatonin is widely accepted as improving sleep patterns and decreasing sleep onset latency [261]. Further studies involving both genotoxicity and cardiac toxicity testing are needed to ensure JP153 does not pose risks associated with these specific adverse effects. 
Table 6-4. CYP450 enzyme interaction studies: JP-153 versus control inhibitors.

\begin{tabular}{lccccc}
\hline $\begin{array}{l}\text { CYP Enzyme } \\
+ \text { Inhibitor }\end{array}$ & $\begin{array}{c}\text { 3A4 } \\
\text { ketoconazole }\end{array}$ & $\begin{array}{c}\mathbf{2 C 9} \\
\text { sulphaphenazole }\end{array}$ & $\begin{array}{c}\text { 2D6 } \\
\text { quinidine }\end{array}$ & $\begin{array}{c}\mathbf{2 C 1 9} \\
\text { ticlopidine }\end{array}$ & $\begin{array}{c}\text { 1A2 } \\
\alpha \text {-naphthoflavone }\end{array}$ \\
\hline Control & $95 \%$ & $88 \%$ & $92 \%$ & $96 \%$ & $94 \%$ \\
JP-153 & $5 \%$ & $40 \%$ & $15 \%$ & $32 \%$ & $12 \%$ \\
\hline
\end{tabular}


Table 6-5. Off-target screen with JP-153.

\begin{tabular}{|c|c|c|}
\hline Receptor & $\%$ Interaction & SD \\
\hline$\alpha 1$ & 12.6 & \pm 7.8 \\
\hline$\alpha 2$ & 6.0 & \pm 7.8 \\
\hline$\beta 1$ & -0.1 & \pm 5.8 \\
\hline$\beta 2$ & 2.1 & \pm 2.4 \\
\hline AT1 & 2.5 & \pm 9.8 \\
\hline BZD & -30.4 & \pm 23.5 \\
\hline B2 & 4.5 & \pm 5.3 \\
\hline CB1 & 1.6 & \pm 5.9 \\
\hline CCK1 & 21.0 & \pm 0.6 \\
\hline D1 & -1.1 & \pm 7.1 \\
\hline D2S & 17.8 & \pm 5.7 \\
\hline ETA & -2.7 & \pm 3.8 \\
\hline GABA & 2.5 & \pm 17.3 \\
\hline GAL2 & -1.3 & \pm 3.0 \\
\hline CXCR2 & -2.1 & \pm 8.2 \\
\hline CCR1 & 6.2 & \pm 10.2 \\
\hline H1 & 4.6 & \pm 14.8 \\
\hline $\mathrm{H} 2$ & -15.1 & \pm 10.3 \\
\hline MC4 & -10.0 & \pm 3.5 \\
\hline MT1 & 56.8 & \pm 4.6 \\
\hline M1 & -21.4 & \pm 16.5 \\
\hline M2 & -15.9 & \pm 18.2 \\
\hline M3 & 1.1 & \pm 10.5 \\
\hline NK2 & 9.6 & \pm 5.4 \\
\hline NK3 & 6.6 & \pm 0.7 \\
\hline Y1 & -12.1 & \pm 3.0 \\
\hline Y2 & -24.1 & \pm 12.9 \\
\hline NTS1 & -19.8 & \pm 9.5 \\
\hline$\delta 2$ & 9.4 & \pm 5.7 \\
\hline$\kappa(\mathrm{KOP})$ & 20.7 & \pm 11.0 \\
\hline$\mu(\mathrm{MOP})$ & 3.9 & \pm 0.1 \\
\hline NOP & -1.5 & \pm 8.6 \\
\hline EP4 & 17.6 & \pm 10.6 \\
\hline 5-HT1A & 14.5 & \pm 7.5 \\
\hline 5-HT1B & -26.8 & \pm 0.5 \\
\hline 5-HT2A & 36.8 & \pm 6.0 \\
\hline 5-HT2B & 75.5 & \pm 5.4 \\
\hline 5-HT6 & 45.5 & \pm 2.4 \\
\hline VPAC1 & 2.9 & \pm 5.0 \\
\hline Norepinephrin & 16.8 & \pm 12.3 \\
\hline Dopamine & 15.2 & \pm 3.4 \\
\hline
\end{tabular}

Notes: Bold: receptors with $>50 \%$ interaction; SD: standard deviation. 
(A.) $\quad \mathrm{MT}_{1}\left(\mathrm{ML}_{1 \mathrm{~A}}\right)$

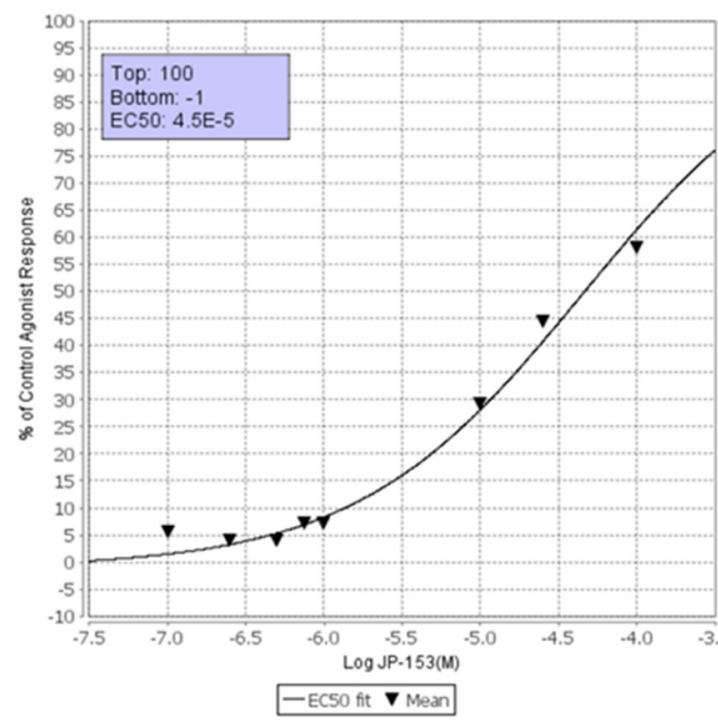

JP-153 on $\mathrm{MT}_{1}\left(\mathrm{ML}_{1 \mathrm{~A}}\right)(h)$

(B.) (agonist effect)

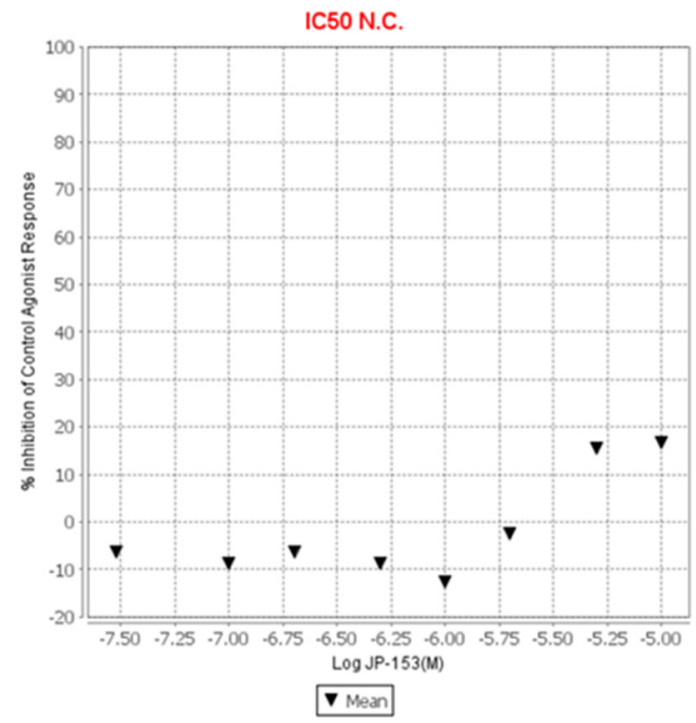

JP-153 on $\mathrm{MT}_{1}\left(\mathrm{ML}_{1 \mathrm{~A}}\right)$ (h) (antagonist effect)
(C.) $5-\mathrm{HT}_{2 \mathrm{~B}}$

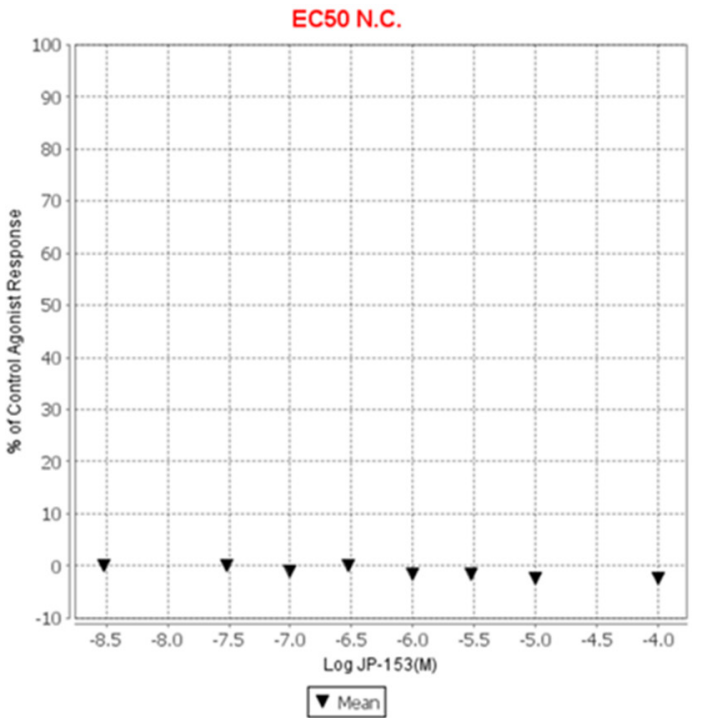

JP-153 on $5-\mathrm{HT}_{2 \mathrm{~B}}(h)$ (agonist effect)

(D.)

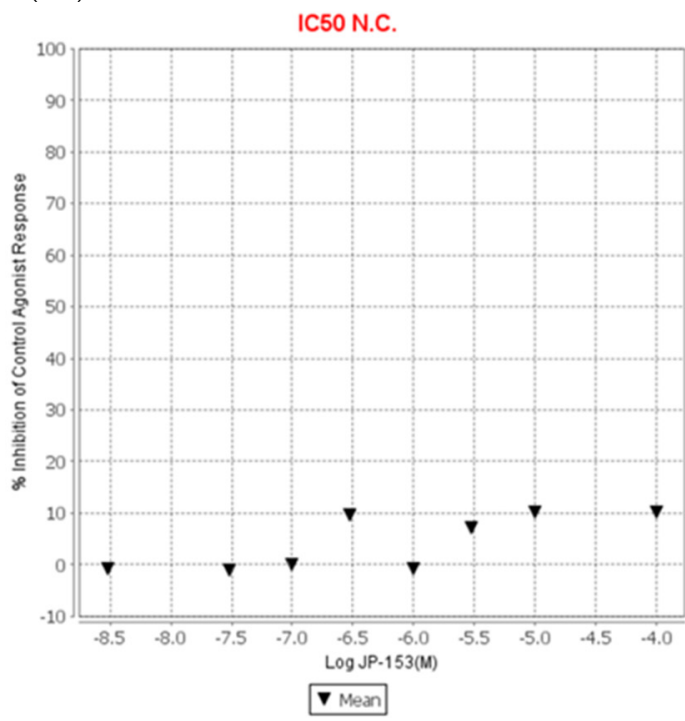

JP-153 on $5-\mathrm{HT}_{2 \mathrm{~B}}(h)$ (antagonist effect)

Figure 6-4. JP-153 EC50 and IC50 analysis against $\mathrm{MT}_{1}$ and 5-HT $2 \mathrm{~B}$ receptors.

A) JP-153 discovered to have agonist behavior with the melatonin-1 MT1 receptor (EC50 $=45 \mu \mathrm{M}$ ) but B) shows no determinable antagonist activity to the receptor. JP-153 is neither an agonist (C) nor antagonist (D) of serotonin 5-HT2B receptors. 


\section{Conclusions}

A sensitive and simple quantification method based on LC-MS/MS to determine, JP-153 in rat plasma after oral and IV administration was developed and validated. This is the first method capable of detecting JP-153 at concentrations in plasma samples after injection in rats over 24 hours. A simple protein precipitation procedure using acetonitrile was used before samples were injected into the autosampler of the LC-MS/MS system. The method showed good precision and accuracy, with moderate to high sensitivity ( $>$ $2.0 \mathrm{ng} / \mathrm{mL}$ in plasma), as well as a wide linear range of $2-1000 \mathrm{ng} / \mathrm{mL}$ in rat plasma. Successful analyses of nearly 200 plasma samples from rats demonstrated that the method is efficient, reliable, and suitable for preclinical and future clinical studies.

A potential concern with any topically applied compound that prevents angiogenesis is significant systemic exposure leading to cardiovascular toxicity, as seen in early usage of anti-VEGF Avastin in patients treated for cancer [262] . Preclinical efficacy studies demonstrated that JP-153 was as effective as topical agents in preventing neovascularization in models of ischemic retinopathy. A key finding in these pharmacokinetic studies was that JP-153 was rapidly cleared, and was $\sim 30 \%$ bioavailable after oral administration. This is an ideal situation for ocular drug therapies as high local concentrations in the eye can be an achievable goal, and once JP-153 crosses into the systemic circulation it will be rapidly eliminated. This finding indicates that if topically applied, JP-153 may not induce any toxicity associated with systemic exposure since its time within the body is limited.

However, these assumptions will need to be validated in ocular pharmacokinetic studies in rabbits to ascertain its ability to cross into the systemic circulation. Rabbits are the selected species for ocular study due to their eye sizes, which allows vitreous fluid to be more accessible. Current ocular PK studies are underway to determine residence time in ocular tissues, and the extent and rate of passage into systemic circulation following ocular administration. If lack of observed cardiovascular effects is noted and coupled with minimal systemic exposure, JP-153 would be an effective and ideal candidate for topical administration for the treatment of neovascular eye disease in humans. 


\section{CHAPTER 7. SUMMARY}

BXD mice have never been used to discover gene variants or quantitative trait loci (QTLs) in response to high dose radiation. Based on our key preliminary findings, we are confident there was ample heritable variation between the BXD strains that contributed to individual differences in radiation exposure responses, i.e., MST. We are also confident in our genetic/genomic resources to reveal genetic, molecular, and cellular mechanisms that contribute to inter-strain variability in radiation response which allowed for the identification of potential biological targets and the innovation of novel radiomitigants. In our approach we have uncovered suggestive QTLs that pointed to endothelial progenitor cell markers, angiogenesis-related proteins, and signaling components of cellular survival, growth and injury-induced inflammation.

Since RR and DR share resemblances in pathogenesis during early stages of injury, and both share neovascular components with wet AMD in the later stages of disease progression, it is not surprising then that inflammatory mediators and EPC markers were identified during these correlation analyses in BXD mice exposed to highdoses of ionizing radiation. We tested the hypothesis that adherence of immune cells and progenitor cells mobilized from the bone marrow could contribute to overall survival following TBI, using an inhibitor of adhesion, 6-B345TTQ. From these data generated from BXD studies in Chapter 3, we further characterized inflammatory and angiogenesisrelated pathways in the setting of vascular injury in the retina, especially how it pertained to mechanisms driving the similar injuries of RR and DR. In fact, these suggestive inferences are supported by decades of research linking inflammation in both proliferative diseases and damage repair mechanisms due to acute radiation exposure and diabetes.

In Chapter 4, we demonstrated that radiation induced leukocyte adhesion through mechanisms involving p38 MAPK, p53, and ICAM-1 activation. The quinic acid derivative, and radiomitigator, KZ-41 lessened leukocyte adhesion and paxillindependent proliferation via inhibition of p38-p53-ICAM-1 signaling. Additional phenotypic changes included p38-dependent tyrosine phosphorylation of the focal adhesion scaffolding protein, paxillin (Tyr118). From our findings, we built a strong link between inflammation, focal adhesion turnover, and angiogenesis which drive pathological neovascular disease in humans. We identified paxillin as an important regulator of NV disease which served as a foundation for target/drug discovery in ischemic retinopathy in Chapter 5. We identified compound JP-153 as a potent inhibitor of REC proliferation, which acted by modulating paxillin and thus inhibiting FA complex signaling through Akt. Furthermore, topical application of a JP-153-loaded nanoemulsion inhibited hallmark features of pathologic RNV in a dose dependent manner using the OIR model. Therefore, we have identified and developed a novel class of small molecules aimed at targeting FA protein interactions which are essential for pathological neovascularization in the eye. To our knowledge, this was the first paradigm validating modulation of paxillin with small molecules to inhibit angiogenesis. 
Chapter 6 details, for the first time, an analytical method to detect JP-153 in rat. We characterized the pharmacokinetic parameters of JP-153 after both oral and IV administration. Additionally, we supplemented our findings in vivo with in vitro liver microsomal stability studies show that JP-153 is rapidly metabolized once in systemic circulation. These data will serve as a basis for further development of JP-153 as a topical and systemic therapeutic for subsequent in vivo efficacy studies.

Our results in the above chapters suggest that small molecules can be used to target both inflammatory signaling following genotoxic stressors, i.e., radiation, and to modulate FA protein paxillin; both serving as effective ways to ameliorate acute retinal injury or inflammation, and subsequent pathological RNV in the human eye. More generally, these findings and data not only shed light on important signaling biomarkers of ischemic injury and neovascular disease, but also served to validate drug targets and agents that may be used to combat other hyper-proliferative diseases in humans, i.e., cancer. 


\section{LIST OF REFERENCES}

1. Flamme, I., T. Frolich, and W. Risau, Molecular mechanisms of vasculogenesis and embryonic angiogenesis. J Cell Physiol, 1997. 173(2): p. 206-10.

2. Benjamin, L.E., I. Hemo, and E. Keshet, A plasticity window for blood vessel remodelling is defined by pericyte coverage of the preformed endothelial network and is regulated by PDGF-B and VEGF. Development, 1998. 125(9): p. 1591-8.

3. Asahara, T., et al., Bone marrow origin of endothelial progenitor cells responsible for postnatal vasculogenesis in physiological and pathological neovascularization. Circ Res, 1999. 85(3): p. 221-8.

4. Ribatti, D., et al., Endothelial progenitor cells in health and disease. Histol Histopathol, 2005. 20(4): p. 1351-8.

5. Carmeliet, P., et al., Abnormal blood vessel development and lethality in embryos lacking a single VEGF allele. Nature, 1996. 380(6573): p. 435-9.

6. $\quad$ Zhang, Z.G., et al., Correlation of VEGF and angiopoietin expression with disruption of blood-brain barrier and angiogenesis after focal cerebral ischemia. J Cereb Blood Flow Metab, 2002. 22(4): p. 379-92.

7. $\quad$ Cheng, N., D.M. Brantley, and J. Chen, The ephrins and Eph receptors in angiogenesis. Cytokine Growth Factor Rev, 2002. 13(1): p. 75-85.

8. Lois, N., et al., Endothelial progenitor cells in diabetic retinopathy. Front Endocrinol (Lausanne), 2014. 5: p. 44.

9. $\quad$ Brunner, S., et al., Correlation of different circulating endothelial progenitor cells to stages of diabetic retinopathy: first in vivo data. Invest Ophthalmol Vis Sci, 2009. 50(1): p. 392-8.

10. Goon, P.K. and G.Y. Lip, Involvement of circulating endothelial progenitor cells and vasculogenic factors in the pathogenesis of diabetic retinopathy. Eye (Lond), 2007. 21(6): p. 838-9; author reply 838.

11. Erickson, M., Medical Eye Illustrations. 2016: jirehdesign.com.

12. Ammendola, M., et al., Targeting endothelial progenitor cells in cancer as a novel biomarker and anti-angiogenic therapy. Curr Stem Cell Res Ther, 2015. 10(2): p. 181-7.

13. Moschetta, M., et al., Role of endothelial progenitor cells in cancer progression. Biochim Biophys Acta, 2014. 1846(1): p. 26-39.

14. Panes, J., et al., Role of leukocyte-endothelial cell adhesion in radiation-induced microvascular dysfunction in rats. Gastroenterology, 1995. 108(6): p. 1761-9.

15. Hiroshiba, N., et al., Radiation-induced leukocyte entrapment in the rat retinal microcirculation. Invest Ophthalmol Vis Sci, 1999. 40(6): p. 1217-22.

16. Joussen, A.M., et al., Leukocyte-mediated endothelial cell injury and death in the diabetic retina. Am J Pathol, 2001. 158(1): p. 147-52.

17. Chen, S., et al., Rolling and transient tethering of leukocytes on antibodies reveal specializations of selectins. Proc Natl Acad Sci U S A, 1997. 94(7): p. 3172-7.

18. Burns, A.R., et al., P-selectin mediates neutrophil adhesion to endothelial cell borders. J Leukoc Biol, 1999. 65(3): p. 299-306. 
19. Yusuf-Makagiansar, H., et al., Inhibition of LFA-1/ICAM-1 and VLA-4/VCAM-1 as a therapeutic approach to inflammation and autoimmune diseases. Med Res Rev, 2002. 22(2): p. 146-67.

20. Funatsu, H., et al., Vitreous levels of vascular endothelial growth factor and intercellular adhesion molecule 1 are related to diabetic macular edema. Ophthalmology, 2005. 112(5): p. 806-16.

21. Ishida, S., et al., Leukocytes mediate retinal vascular remodeling during development and vaso-obliteration in disease. Nat Med, 2003. 9(6): p. 781-8.

22. Miyamoto, K., et al., Prevention of leukostasis and vascular leakage in streptozotocin-induced diabetic retinopathy via intercellular adhesion molecule-1 inhibition. Proc Natl Acad Sci U S A, 1999. 96(19): p. 10836-41.

23. Brown, G.C., et al., Radiation retinopathy. Ophthalmology, 1982. 89(12): p. 1494-501.

24. Zamber, R.W. and J.L. Kinyoun, Radiation retinopathy. West J Med, 1992. 157(5): p. 530-3.

25. Yuan, H., et al., Radiation-induced permeability and leukocyte adhesion in the rat blood-brain barrier: modulation with anti-ICAM-1 antibodies. Brain Res, 2003. 969(1-2): p. 59-69.

26. Joussen, A.M., et al., A central role for inflammation in the pathogenesis of diabetic retinopathy. FASEB J, 2004. 18(12): p. 1450-2.

27. Schulze-Osthoff, K., et al., Apoptosis signaling by death receptors. Eur J Biochem, 1998. 254(3): p. 439-59.

28. Nagata, S. and P. Golstein, The Fas death factor. Science, 1995. 267(5203): p. 1449-56.

29. Joussen, A.M., et al., Suppression of Fas-FasL-induced endothelial cell apoptosis prevents diabetic blood-retinal barrier breakdown in a model of streptozotocininduced diabetes. FASEB J, 2003. 17(1): p. 76-8.

30. Fong, D.S., et al., Retinopathy in diabetes. Diabetes Care, 2004. 27 Suppl 1: p. S84-7.

31. Kreuger, J. and M. Phillipson, Targeting vascular and leukocyte communication in angiogenesis, inflammation and fibrosis. Nat Rev Drug Discov, 2016. 15(2): p. $125-42$.

32. Kaushik, M., et al., Risk of radiation retinopathy in patients with orbital and ocular lymphoma. Int J Radiat Oncol Biol Phys, 2012. 84(5): p. 1145-50.

33. Figueroa, M.S., C. Arruabarrena, and M. Sales-Sanz, New Treatments in Radiation Retinopathy. Retinal Cases and Brief Reports, 2011. 5(2): p. 171-174 10.1097/ICB.0b013e3181d427ed.

34. Giuliari, G.P., et al., Current treatments for radiation retinopathy. Acta Oncologica, 2011. 50(1): p. 6-13.

35. Finger, P.T., K.J. Chin, and G. Duvall, Palladium-103 ophthalmic plaque radiation therapy for choroidal melanoma: 400 treated patients. Ophthalmology, 2009. 116(4): p. 790-6, $796 \mathrm{e} 1$.

36. Gragoudas, E.S., et al., Long-term results of proton beam irradiated uveal melanomas. Ophthalmology, 1987. 94(4): p. 349-53. 
37. Haas, A., et al., Incidence of radiation retinopathy after high-dosage singlefraction gamma knife radiosurgery for choroidal melanoma. Ophthalmology, 2002. 109(5): p. 909-13.

38. Krema, H., et al., Stereotactic radiotherapy for treatment of juxtapapillary choroidal melanoma: 3-year follow-up. Br J Ophthalmol, 2009. 93(9): p. 1172-6.

39. Levy, R.P., et al., Heavy-charged-particle radiosurgery of the pituitary gland: clinical results of 840 patients. Stereotact Funct Neurosurg, 1991. 57(1-2): p. 2235.

40. Parsons, J.T., et al., Radiation retinopathy after external-beam irradiation: analysis of time-dose factors. Int J Radiat Oncol Biol Phys, 1994. 30(4): p. $765-$ 73.

41. Bianciotto, C., et al., Proliferative radiation retinopathy after plaque radiotherapy for uveal melanoma. Ophthalmology, 2010. 117(5): p. 1005-12.

42. Gunduz, K., et al., Radiation complications and tumor control after plaque radiotherapy of choroidal melanoma with macular involvement. Am J Ophthalmol, 1999. 127(5): p. 579-89.

43. Shields, C.L., et al., Plaque radiotherapy for large posterior uveal melanomas $>$ or $=8$-mm thick) in 354 consecutive patients. Ophthalmology, 2002. 109(10): p. 1838-49.

44. Phillpotts, B.A., et al., Uveal melanomas in black patients: a case series and comparative review. J Natl Med Assoc, 1995. 87(9): p. 709-14.

45. Shields, C.L., et al., Uveal melanoma in teenagers and children. A report of 40 cases. Ophthalmology, 1991. 98(11): p. 1662-6.

46. Poulaki, V., et al., Acute intensive insulin therapy exacerbates diabetic bloodretinal barrier breakdown via hypoxia-inducible factor-1alpha and VEGF. J Clin Invest, 2002. 109(6): p. 805-15.

47. Zhang, Q., C. Soderland, and J.J. Steinle, Regulation of retinal endothelial cell apoptosis through activation of the IGFBP-3 receptor. Apoptosis, 2013. 18(3): p. 361-8.

48. Gerber, H.P., et al., Vascular endothelial growth factor regulates endothelial cell survival through the phosphatidylinositol 3'-kinase/Akt signal transduction pathway. Requirement for Flk-1/KDR activation. J Biol Chem, 1998. 273(46): p. 30336-43.

49. Suzuma, K., et al., Vascular endothelial growth factor induces expression of connective tissue growth factor via KDR, Flt1, and phosphatidylinositol 3-kinaseakt-dependent pathways in retinal vascular cells. J Biol Chem, 2000. 275(52): p. 40725-31.

50. Waldbillig, R.J., et al., Evidence for an insulin-like growth factor autocrineparacrine system in the retinal photoreceptor-pigment epithelial cell complex. $\mathrm{J}$ Neurochem, 1991. 57(5): p. 1522-33.

51. Seigel, G.M., et al., Systemic IGF-I treatment inhibits cell death in diabetic rat retina. J Diabetes Complications, 2006. 20(3): p. 196-204.

52. Bergers, G. and D. Hanahan, Modes of resistance to anti-angiogenic therapy. Nat Rev Cancer, 2008. 8(8): p. 592-603.

53. Boyle, E., Folkman: Angiogenesis research rooted in ophthalmology, in Ocular Surgery News. 2007. 
54. Karmel, M., Off-Label Anti-VEGF Therapy: Targeting New Conditions, in EyeNet Magazine. 2012, American Academy of Ophthalmology.

55. Ng, Y.S., et al., Differential expression of VEGF isoforms in mouse during development and in the adult. Dev Dyn, 2001. 220(2): p. 112-21.

56. Kim, L.A. and P.A. D'Amore, A brief history of anti-VEGF for the treatment of ocular angiogenesis. Am J Pathol, 2012. 181(2): p. 376-9.

57. van Beijnum, J.R., et al., The great escape; the hallmarks of resistance to antiangiogenic therapy. Pharmacol Rev, 2015. 67(2): p. 441-61.

58. Yang, X. and C.L. Cepko, Flk-1, a receptor for vascular endothelial growth factor (VEGF), is expressed by retinal progenitor cells. J Neurosci, 1996. 16(19): p. 6089-99.

59. Grimm, C., et al., Neuroprotection by hypoxic preconditioning: HIF-1 and erythropoietin protect from retinal degeneration. Semin Cell Dev Biol, 2005. 16(4-5): p. 531-8.

60. Saint-Geniez, M., et al., An essential role for RPE-derived soluble VEGF in the maintenance of the choriocapillaris. Proc Natl Acad Sci U S A, 2009. 106(44): p. 18751-6.

61. Saint-Geniez, M., et al., Endogenous VEGF is required for visual function: evidence for a survival role on muller cells and photoreceptors. PLoS One, 2008. 3(11): p. e3554.

62. Pardanaud, L., et al., Two distinct endothelial lineages in ontogeny, one of them related to hemopoiesis. Development, 1996. 122(5): p. 1363-71.

63. Zhao, H., et al., Mobilization of Circulating Endothelial Progenitor Cells by dl-3n-Butylphthalide in Acute Ischemic Stroke Patients. J Stroke Cerebrovasc Dis, 2016. 25(4): p. 752-60.

64. Shintani, S., et al., Mobilization of endothelial progenitor cells in patients with acute myocardial infarction. Circulation, 2001. 103(23): p. 2776-9.

65. Moore, M.A., et al., Mobilization of endothelial and hematopoietic stem and progenitor cells by adenovector-mediated elevation of serum levels of SDF-1, $V E G F$, and angiopoietin-1. Ann N Y Acad Sci, 2001. 938: p. 36-45; discussion 45-7.

66. Butcher, E.C. and L.J. Picker, Lymphocyte homing and homeostasis. Science, 1996. 272(5258): p. 60-6.

67. Springer, T.A., Traffic signals for lymphocyte recirculation and leukocyte emigration: the multistep paradigm. Cell, 1994. 76(2): p. 301-14.

68. Qin, G., et al., Functional disruption of alpha4 integrin mobilizes bone marrowderived endothelial progenitors and augments ischemic neovascularization. J Exp Med, 2006. 203(1): p. 153-63.

69. Sunguroglu, A., et al., PXN and TRIO are elevated in CD133+ GSCs transcriptomically. The FASEB Journal, 2016. 30(1 Supplement): p. lb96-lb96.

70. Garcia-Barros, M., et al., Tumor response to radiotherapy regulated by endothelial cell apoptosis. Science, 2003. 300(5622): p. 1155-9.

71. Ziegelhoeffer, T., et al., Bone marrow-derived cells do not incorporate into the adult growing vasculature. Circ Res, 2004. 94(2): p. 230-8.

72. Hill, J.M., et al., Circulating endothelial progenitor cells, vascular function, and cardiovascular risk. N Engl J Med, 2003. 348(7): p. 593-600. 
73. Abedi, H. and I. Zachary, Vascular endothelial growth factor stimulates tyrosine phosphorylation and recruitment to new focal adhesions of focal adhesion kinase and paxillin in endothelial cells. J Biol Chem, 1997. 272(24): p. 15442-51.

74. Provenzano, P.P. and P.J. Keely, Mechanical signaling through the cytoskeleton regulates cell proliferation by coordinated focal adhesion and Rho GTPase signaling. J Cell Sci, 2011. 124(Pt 8): p. 1195-205.

75. Waltenberger, J., et al., Different signal transduction properties of KDR and Flt1, two receptors for vascular endothelial growth factor. J Biol Chem, 1994. 269(43): p. 26988-95.

76. Brown, M.C., et al., Src and FAK kinases cooperate to phosphorylate paxillin kinase linker, stimulate its focal adhesion localization, and regulate cell spreading and protrusiveness. Mol Biol Cell, 2005. 16(9): p. 4316-28.

77. Brown, M.C. and C.E. Turner, Paxillin: adapting to change. Physiol Rev, 2004. 84(4): p. 1315-39.

78. $\quad$ Eliceiri, B.P., et al., Selective requirement for Src kinases during VEGF-induced angiogenesis and vascular permeability. Mol Cell, 1999. 4(6): p. 915-24.

79. Richardson, A., et al., Inhibition of cell spreading by expression of the C-terminal domain of focal adhesion kinase (FAK) is rescued by coexpression of Src or catalytically inactive FAK: a role for paxillin tyrosine phosphorylation. Mol Cell Biol, 1997. 17(12): p. 6906-14.

80. Lamorte, L., et al., Crk associates with a multimolecular Paxillin/GIT2/beta-PIX complex and promotes Rac-dependent relocalization of Paxillin to focal contacts. Mol Biol Cell, 2003. 14(7): p. 2818-31.

81. Bertolucci, C., et al., Daily rhythms of serum leptin in ewes: effects of feeding, pregnancy and lactation. Chronobiol Int, 2005. 22(5): p. 817-27.

82. Scheswohl, D.M., et al., Multiple paxillin binding sites regulate FAK function. J Mol Signal, 2008. 3: p. 1.

83. Turner, C.E., Paxillin interactions. J Cell Sci, 2000. 113 Pt 23: p. 4139-40.

84. Brown, M.C., M.S. Curtis, and C.E. Turner, Paxillin LD motifs may define a new family of protein recognition domains. Nat Struct Biol, 1998. 5(8): p. 677-8.

85. Vanarotti, M.S., et al., Structural and mechanistic insights into the interaction between Pyk2 and paxillin LD motifs. J Mol Biol, 2014. 426(24): p. 3985-4001.

86. Arold, S.T., M.K. Hoellerer, and M.E. Noble, The structural basis of localization and signaling by the focal adhesion targeting domain. Structure, 2002. 10(3): $\mathrm{p}$. 319-27.

87. Weis, S.M., et al., Compensatory role for Pyk2 during angiogenesis in adult mice lacking endothelial cell FAK. J Cell Biol, 2008. 181(1): p. 43-50.

88. Thompson, K.E., et al., Quinic Acid Derivative KZ-41 Exhibits Radiomitigating Activity in Preclinical Models of Radiation Injury. Drug Discovery Research, 2014. (in press).

89. Yates, C.R., Miller, D.D., Gaber, M.W., Thompson, K.E., Zeng, K., Toutounchian, J.J., Anti-Inflammatory Quinic Acid Derivatives for Radioprotection/Radiomitigation. November 8, 2012: US Patent 2012/0283331 A1. 
90. Zhang, Q., et al., Novel quinic acid derivative KZ-41 prevents retinal endothelial cell apoptosis without inhibiting retinoblastoma cell death through p38 signaling. Invest Ophthalmol Vis Sci, 2013. 54(9): p. 5937-43.

91. Li Calzi, S., et al., Endothelial progenitor dysfunction in the pathogenesis of diabetic retinopathy: treatment concept to correct diabetes-associated deficits. EPMA J, 2010. 1(1): p. 88-100.

92. Taylor, B.A., et al., Genotyping new BXD recombinant inbred mouse strains and comparison of BXD and consensus maps. Mamm Genome, 1999. 10(4): p. 33548.

93. Peirce, M.A., et al., Pathology associated with endogenous development of haematozoa in birds from southeast Queensland. Avian Pathol, 2004. 33(4): p. 445-50.

94. Peirce, J.L., et al., A new set of $B X D$ recombinant inbred lines from advanced intercross populations in mice. BMC Genet, 2004. 5: p. 7.

95. Miyairi, I., et al., The 477 GTPases Iigp2 and Irgb10 regulate innate immunity and inflammation to murine Chlamydia psittaci infection. J Immunol, 2007. 179(3): p. 1814-24.

96. Aziz, R.K., et al., Susceptibility to severe Streptococcal sepsis: use of a large set of isogenic mouse lines to study genetic and environmental factors. Genes Immun, 2007. 8(5): p. 404-15.

97. Mozhui, K., et al., Dissection of a QTL hotspot on mouse distal chromosome 1 that modulates neurobehavioral phenotypes and gene expression. PLoS Genet, 2008. 4(11): p. e1000260.

98. Lu, L., et al., Using gene expression databases for classical trait QTL candidate gene discovery in the $B X D$ recombinant inbred genetic reference population: mouse forebrain weight. BMC Genomics, 2008. 9: p. 444.

99. Rosen, G.D., et al., Genetic modulation of striatal volume by loci on Chrs 6 and 17 in BXD recombinant inbred mice. Genes Brain Behav, 2009. 8(3): p. 296-308.

100. Wang, J., R.W. Williams, and K.F. Manly, WebQTL: web-based complex trait analysis. Neuroinformatics, 2003. 1(4): p. 299-308.

101. Overall, R.W., et al., Genetics of the hippocampal transcriptome in mouse: a systematic survey and online neurogenomics resource. Front Neurosci, 2009. 3: p. 55.

102. Churchill, G.A. and R.W. Doerge, Empirical threshold values for quantitative trait mapping. Genetics, 1994. 138(3): p. 963-71.

103. Shete, S., et al., Effect of winsorization on power and type 1 error of variance components and related methods of QTL detection. Behav Genet, 2004. 34(2): p. 153-9.

104. Yale, C. and A.B. Forsythe, Winsorized Regression. Technometrics, 1976. 18(3): p. 291-300.

105. Hasegawa, M., et al., Progenitor cell mobilization by granulocyte colonystimulating factor controlled by loci on chromosomes 2 and 11. Blood, 2000. 95(5): p. 1872-4.

106. Bystrykh, L., et al., Uncovering regulatory pathways that affect hematopoietic stem cell function using 'genetical genomics'. Nat Genet, 2005. 37(3): p. 225-32. 
107. Gerrits, A., et al., Expression quantitative trait loci are highly sensitive to cellular differentiation state. PLoS Genet, 2009. 5(10): p. e1000692.

108. Lawrence, M.B., L.V. McIntire, and S.G. Eskin, Effect of flow on polymorphonuclear leukocytelendothelial cell adhesion. Blood, 1987. 70(5): p. 1284-90.

109. Toutounchian, J.J., et al., Modulation of radiation injury response in retinal endothelial cells by quinic acid derivative KZ-41 involves p38 MAPK. PLoS One, 2014. 9(6): p. e100210.

110. Roderick, T.H., The Response of Twenty-Seven Inbred Strains of Mice to Daily Doses of Whole-Body X-Irradiation. Radiat Res, 1963. 20: p. 631-9.

111. Takahashi, J.S., L.H. Pinto, and M.H. Vitaterna, Forward and reverse genetic approaches to behavior in the mouse. Science, 1994. 264(5166): p. 1724-33.

112. D'Arena, G., et al., Immunophenotypic profile of AC133-positive cells in bone marrow, mobilized peripheral blood and umbilical cord blood. Leuk Lymphoma, 2002. 43(4): p. 869-73.

113. Stewart, M., M. Thiel, and N. Hogg, Leukocyte integrins. Curr Opin Cell Biol, 1995. 7(5): p. 690-6.

114. Shimizu, Y., D.M. Rose, and M.H. Ginsberg, Integrins in the immune system. Adv Immunol, 1999. 72: p. 325-80.

115. Jing, D., et al., CD49d blockade by natalizumab in patients with multiple sclerosis affects steady-state hematopoiesis and mobilizes progenitors with a distinct phenotype and function. Bone Marrow Transplant, 2010. 45(10): p. 1489-96.

116. Feral, C.C., et al., Blocking the alpha 4 integrin-paxillin interaction selectively impairs mononuclear leukocyte recruitment to an inflammatory site. J Clin Invest, 2006. 116(3): p. 715-23.

117. Kummer, C., et al., A small molecule that inhibits the interaction of paxillin and alpha 4 integrin inhibits accumulation of mononuclear leukocytes at a site of inflammation. J Biol Chem, 2010. 285(13): p. 9462-9.

118. Liu, S., et al., Binding of paxillin to alpha4 integrins modifies integrin-dependent biological responses. Nature, 1999. 402(6762): p. 676-81.

119. Toulany, M., et al., Akt promotes post-irradiation survival of human tumor cells through initiation, progression, and termination of DNA-PKcs-dependent DNA double-strand break repair. Mol Cancer Res, 2012. 10(7): p. 945-57.

120. Gaur, U. and B.B. Aggarwal, Regulation of proliferation, survival and apoptosis by members of the TNF superfamily. Biochem Pharmacol, 2003. 66(8): p. 1403-8.

121. Zeng, K., et al., Synthesis and biological evaluation of quinic acid derivatives as anti-inflammatory agents. Bioorg Med Chem Lett, 2009. 19(18): p. 5458-60.

122. Koturbash, I., et al., Irradiation induces DNA damage and modulates epigenetic effectors in distant bystander tissue in vivo. Oncogene, 2006. 25(31): p. 4267-75.

123. Giri, P.G., et al., Comparison of single, fractionated and hyperfractionated irradiation on the development of normal tissue damage in rat lung. Int $\mathrm{J}$ Radiat Oncol Biol Phys, 1985. 11(3): p. 527-34.

124. Lam, G.K., et al., Method of analysis to derive cell survival from observation of tissue damage following fractionated irradiation. Radiat Res, 1979. 77(3): p. 44052. 
125. Chen, P.J. and Y.S. Huang, CPEB2-eEF2 interaction impedes HIF-1alpha RNA translation. EMBO J, 2012. 31(4): p. 959-71.

126. Ghai, R., et al., Clq and its growing family. Immunobiology, 2007. 212(4-5): p. 253-66.

127. Prechl, J. and L. Czirjak, The endothelial deprotection hypothesis for lupus pathogenesis: the dual role of Clq as a mediator of clearance and regulator of endothelial permeability. F1000Res, 2015. 4: p. 24.

128. Feng, X., et al., Cooperation of Clq receptors and integrins in Clq-mediated endothelial cell adhesion and spreading. J Immunol, 2002. 168(5): p. 2441-8.

129. Ferrero, E., F. Saccucci, and F. Malavasi, The human CD38 gene: polymorphism, $C p G$ island, and linkage to the CD157 (BST-1) gene. Immunogenetics, 1999. 49(7-8): p. 597-604.

130. Bong, J.J., et al., IDENTIFICATION OF GENES EXPRESSED IN LOW-DOSERATE $\gamma$-IRRADIATED MOUSE WHOLE BRAIN. Journal of Radiation Protection and Research, 2013.38(4): p. 166-171.

131. Tassi, E., et al., Impact of Fibroblast Growth Factor-Binding Protein-1 Expression on Angiogenesis and Wound Healing. The American Journal of Pathology, 2011. 179(5): p. 2220-2232.

132. Schafer, M. and S. Werner, Cancer as an overhealing wound: an old hypothesis revisited. Nat Rev Mol Cell Biol, 2008. 9(8): p. 628-638.

133. Peplow, P.V., Growth factor- and cytokine-stimulated endothelial progenitor cells in post-ischemic cerebral neovascularization. Neural Regen Res, 2014. 9(15): p. 1425-9.

134. Wen, L.P., et al., Cleavage of focal adhesion kinase by caspases during apoptosis. J Biol Chem, 1997. 272(41): p. 26056-61.

135. Carragher, N.O., et al., Cleavage of Focal Adhesion Kinase by Different Proteases during Src-regulated Transformation and Apoptosis DISTINCT ROLES FOR CALPAIN AND CASPASES. Journal of Biological Chemistry, 2001. 276(6): p. $4270-4275$.

136. Lapidot, T. and I. Petit, Current understanding of stem cell mobilization: the roles of chemokines, proteolytic enzymes, adhesion molecules, cytokines, and stromal cells. Exp Hematol, 2002. 30(9): p. 973-81.

137. Chen, M. and $\mathrm{H} . \mathrm{Xu}$, Parainflammation, chronic inflammation, and age-related macular degeneration. J Leukoc Biol, 2015. 98(5): p. 713-25.

138. Ito, A., et al., A truncated isoform of the PP2A B56 subunit promotes cell motility through paxillin phosphorylation. EMBO J, 2000. 19(4): p. 562-71.

139. Zhu, J., et al., Focal adhesion kinase signaling pathway participates in the formation of choroidal neovascularization and regulates the proliferation and migration of choroidal microvascular endothelial cells by acting through HIF-1 and VEGF expression in RPE cells. Experimental Eye Research, 2009. 88(5): p. 910-918.

140. Jampol, L.M., et al., The COMS randomized trial of iodine 125 brachytherapy for choroidal melanoma: $I V$. Local treatment failure and enucleation in the first 5 years after brachytherapy. COMS report no. 19. Ophthalmology, 2002. 109(12): p. 2197-206. 
141. Diener-West, M., et al., The COMS randomized trial of iodine 125 brachytherapy for choroidal melanoma, III: initial mortality findings. COMS Report No. 18. Arch Ophthalmol, 2001. 119(7): p. 969-82.

142. Avery, R.B., et al., Histopathologic characteristics of choroidal melanoma in eyes enucleated after iodine 125 brachytherapy in the collaborative ocular melanoma study. Arch Ophthalmol, 2008. 126(2): p. 207-12.

143. Bulavin, D.V., et al., Phosphorylation of human 553 by p38 kinase coordinates $N$ terminal phosphorylation and apoptosis in response to UV radiation. EMBO J, 1999. 18(23): p. 6845-54.

144. Gorgoulis, V.G., et al., p53 activates ICAM-1 (CD54) expression in an NFkappaB-independent manner. EMBO J, 2003. 22(7): p. 1567-78.

145. Huang, C., et al., p38 kinase mediates $U V$-induced phosphorylation of $p 53$ protein at serine 389. J Biol Chem, 1999. 274(18): p. 12229-35.

146. She, Q.B., N. Chen, and Z. Dong, ERKs and p38 kinase phosphorylate 553 protein at serine 15 in response to UV radiation. J Biol Chem, 2000. 275(27): p. 20444-9.

147. Wang, X. and T. Ohnishi, p53-dependent signal transduction induced by stress. J Radiat Res, 1997. 38(3): p. 179-94.

148. Siliciano, J.D., et al., DNA damage induces phosphorylation of the amino terminus of p53. Genes Dev, 1997. 11(24): p. 3471-81.

149. Sanchez-Prieto, R., et al., A role for the $p 38$ mitogen-acitvated protein kinase pathway in the transcriptional activation of 553 on genotoxic stress by chemotherapeutic agents. Cancer Res, 2000. 60(9): p. 2464-72.

150. Sundstrom, C. and K. Nilsson, Establishment and characterization of a human histiocytic lymphoma cell line (U-937). Int J Cancer, 1976. 17(5): p. 565-77.

151. Davies, S.P., et al., Specificity and mechanism of action of some commonly used protein kinase inhibitors. Biochem J, 2000. 351(Pt 1): p. 95-105.

152. Yu, Y.M., et al., Ellagic acid inhibits IL-1beta-induced cell adhesion molecule expression in human umbilical vein endothelial cells. Br J Nutr, 2007. 97(4): p. 692-8.

153. Chang, W.C., et al., Chlorogenic acid attenuates adhesion molecules upregulation in IL-1beta-treated endothelial cells. Eur J Nutr, 2010. 49(5): p. 267-75.

154. Frangos, J.A., L.V. McIntire, and S.G. Eskin, Shear stress induced stimulation of mammalian cell metabolism. Biotechnol Bioeng, 1988. 32(8): p. 1053-1060.

155. Wagers, A.J., et al., Interleukin 12 and Interleukin 4 Control T Cell Adhesion to Endothelial Selectins through Opposite Effects on a1,3-fucosyltransferase VII Gene Expression. The Journal of Experimental Medicine, 1998. 188(12): p. 22252231.

156. McCarty, O.J., et al., Immobilized platelets support human colon carcinoma cell tethering, rolling, and firm adhesion under dynamic flow conditions. Blood, 2000. 96(5): p. 1789-97.

157. Alon, R., et al., Interactions through L-selectin between leukocytes and adherent leukocytes nucleate rolling adhesions on selectins and VCAM-1 in shear flow. J Cell Biol, 1996. 135(3): p. 849-65. 
158. Li, J., et al., Application of glutaraldehyde to in-cell Western assay for normalization. Anal Biochem, 2010. 398(2): p. 254-6.

159. Ammar, H.O., et al., Nanoemulsion as a potential ophthalmic delivery system for dorzolamide hydrochloride. AAPS PharmSciTech, 2009. 10(3): p. 808-19.

160. Shafiq-un-Nabi, S., et al., Formulation development and optimization using nanoemulsion technique: a technical note. AAPS PharmSciTech, 2007. 8(2): p. Article 28.

161. Smith, L.E., et al., Oxygen-induced retinopathy in the mouse. Invest Ophthalmol Vis Sci, 1994. 35(1): p. 101-11.

162. Zhang, Q., et al., Activation of cytosolic phospholipase A2 downstream of the Srcphospholipase D1 (PLD1)-protein kinase C gamma (PKCgamma) signaling axis is required for hypoxia-induced pathological retinal angiogenesis. J Biol Chem, 2011. 286(25): p. 22489-98.

163. Arnold, T.D., et al., Defective retinal vascular endothelial cell development as a consequence of impaired integrin alphaVbeta8-mediated activation of transforming growth factor-beta. J Neurosci, 2012. 32(4): p. 1197-206.

164. Connor, K.M., et al., Quantification of oxygen-induced retinopathy in the mouse: a model of vessel loss, vessel regrowth and pathological angiogenesis. Nat Protoc, 2009. 4(11): p. 1565-73.

165. Stahl, A., et al., Computer-aided quantification of retinal neovascularization. Angiogenesis, 2009. 12(3): p. 297-301.

166. Zeng, K., et al., Preclinical pharmacokinetics of the radiomitigator KZ-41 in rats. Xenobiotica, 2011. 41(11): p. 1006-12.

167. He, H., et al., A sensitive and fast LC-MS/MS method for determination of betareceptor agonist JP-49b: application to a pharmacokinetic study in rats. $\mathrm{J}$ Chromatogr B Analyt Technol Biomed Life Sci, 2014. 953-954: p. 86-91.

168. Food and Drug Administration. Draft Guidance for Industry: Bioanalytical Method Validation. Rockville, M., U.S Food and Drug Administration, 1999.

169. Kinashi, T., Intracellular signalling controlling integrin activation in lymphocytes. Nat Rev Immunol, 2005. 5(7): p. 546-59.

170. Gaber, M.W., et al., Differences in ICAM-1 and TNF-alpha expression between large single fraction and fractionated irradiation in mouse brain. Int $\mathrm{J}$ Radiat Biol, 2003. 79(5): p. 359-66.

171. Leeuwenberg, J.F., et al., E-selectin and intercellular adhesion molecule-1 are released by activated human endothelial cells in vitro. Immunology, 1992. 77(4): p. 543-9.

172. Derijard, B., et al., Independent human MAP-kinase signal transduction pathways defined by MEK and MKK isoforms. Science, 1995. 267(5198): p. 682-5.

173. Raingeaud, J., et al., Pro-inflammatory cytokines and environmental stress cause p38 mitogen-activated protein kinase activation by dual phosphorylation on tyrosine and threonine. J Biol Chem, 1995. 270(13): p. 7420-6.

174. Meineke, V., et al., Protein kinase inhibitors modulate time-dependent effects of $U V$ and ionizing irradiation on ICAM-1 expression on human hepatoma cells. Int J Radiat Biol, 2002. 78(7): p. 577-83.

175. Yan, W., et al., Role of 38 MAPK in ICAM-1 expression of vascular endothelial cells induced by lipopolysaccharide. Shock, 2002. 17(5): p. 433-8. 
176. Archer, D.B., W.M. Amoaku, and T.A. Gardiner, Radiation retinopathy--clinical, histopathological, ultrastructural and experimental correlations. Eye (Lond), 1991. 5 ( Pt 2): p. 239-51.

177. Zhang, X., et al., Apoptosis and cell proliferation in proliferative retinal disorders: PCNA, Ki-67, caspase-3, and PARP expression. Curr Eye Res, 2005. 30(5): p. 395-403.

178. Archer, D.B. and T.A. Gardiner, Ionizing radiation and the retina. Curr Opin Ophthalmol, 1994. 5(3): p. 59-65.

179. Jung, J.W., et al., Ionising radiation induces changes associated with epithelialmesenchymal transdifferentiation and increased cell motility of A549 lung epithelial cells. Eur J Cancer, 2007. 43(7): p. 1214-24.

180. Rajashekhar, G., et al., Pro-inflammatory angiogenesis is mediated by p38 MAP kinase. J Cell Physiol, 2011. 226(3): p. 800-8.

181. Beinke, C., D. Van Beuningen, and N. Cordes, Ionizing radiation modules of the expression and tyrosine phosphorylation of the focal adhesion-associated proteins focal adhesion kinase (FAK) and its substrates p130cas and paxillin in A549 human lung carcinoma cells in vitro. Int J Radiat Biol, 2003. 79(9): p. 721-31.

182. Bai, Y., et al., MicroRNA-126 inhibits ischemia-induced retinal neovascularization via regulating angiogenic growth factors. Exp Mol Pathol, 2011. 91(1): p. 471-7.

183. Yates, C.R., et al., Methods for Regulating Retinal Endothelial Cell Viability. Provisional US Patent Application 14/074,457, November 7, 2013.

184. Langston, W., et al., Regulation of endothelial glutathione by ICAM-1 governs VEGF-A-mediated eNOS activity and angiogenesis. Free Radic Biol Med, 2007. 42(5): p. 720-9.

185. Radisavljevic, Z., H. Avraham, and S. Avraham, Vascular endothelial growth factor up-regulates ICAM-1 expression via the phosphatidylinositol $3 \mathrm{OH}$ kinase/AKT/Nitric oxide pathway and modulates migration of brain microvascular endothelial cells. J Biol Chem, 2000. 275(27): p. 20770-4.

186. Webb, D.J., et al., FAK-Src signalling through paxillin, ERK and MLCK regulates adhesion disassembly. Nat Cell Biol, 2004. 6(2): p. 154-61.

187. Berginski, M.E., et al., High-Resolution Quantification of Focal Adhesion Spatiotemporal Dynamics in Living Cells. PloS one, 2011. 6(7): p. e22025.

188. Kornberg, L.J., et al., Focal adhesion kinase overexpression induces enhanced pathological retinal angiogenesis. Invest Ophthalmol Vis Sci, 2004. 45(12): p. 4463-9.

189. Abdelsaid, M.A., et al., Early intervention of tyrosine nitration prevents vasoobliteration and neovascularization in ischemic retinopathy. J Pharmacol Exp Ther, 2010. 332(1): p. 125-34.

190. Kanno, S., et al., Roles of two VEGF receptors, Flt-1 and KDR, in the signal transduction of VEGF effects in human vascular endothelial cells. Oncogene, 2000. 19(17): p. 2138-46.

191. Rousseau, S., et al., p38 MAP kinase activation by vascular endothelial growth factor mediates actin reorganization and cell migration in human endothelial cells. Oncogene, 1997. 15(18): p. 2169-77. 
192. Ferris, F.L., et al., AGe-related macular degeneration and blindness due to neovascular maculopathy. Archives of Ophthalmology, 1984. 102(11): p. 16401642.

193. Aiello, L.P., et al., Vascular endothelial growth factor in ocular fluid of patients with diabetic retinopathy and other retinal disorders. N Engl J Med, 1994. 331(22): p. 1480-7.

194. Osborne, N.N., et al., Retinal ischemia: mechanisms of damage and potential therapeutic strategies. Prog Retin Eye Res, 2004. 23(1): p. 91-147.

195. Ozaki, H., et al., Hypoxia inducible factor-1alpha is increased in ischemic retina: temporal and spatial correlation with VEGF expression. Invest Ophthalmol Vis Sci, 1999. 40(1): p. 182-9.

196. Wilkinson-Berka, J.L., et al., Reactive oxygen species, Nox and angiotensin II in angiogenesis: implications for retinopathy. Clin Sci (Lond), 2013. 124(10): $\mathrm{p}$. 597-615.

197. Nowak, J.Z., Age-related macular degeneration (AMD): pathogenesis and therapy. Pharmacol Rep, 2006. 58(3): p. 353-63.

198. Release, F.N. FDA approves Lucentis to treat diabetic retinopathy in patients with diabetic macular edema. [webpage] 2015.

199. Tokunaga, C.C., et al., Effects of anti-VEGF treatment on the recovery of the developing retina following oxygen-induced retinopathy. Invest Ophthalmol Vis Sci, 2014. 55(3): p. 1884-92.

200. Marneros, A.G., et al., Vascular endothelial growth factor expression in the retinal pigment epithelium is essential for choriocapillaris development and visual function. Am J Pathol, 2005. 167(5): p. 1451-9.

201. Boozalis, G.T., A.P. Schachat, and W.R. Green, Subretinal neovascularization from the retina in radiation retinopathy. Retina, 1987. 7(3): p. 156-61.

202. Finger, P.T., Radiation Retinopathy Is Treatable With Anti-Vascular Endothelial Growth Factor Bevacizumab (Avastin). International Journal of Radiation Oncology*Biology*Physics, 2008. 70(4): p. 974-977.

203. Houlden, C.E., G.C. Lloyd-Jones, and K.I. Booker-Milburn, Facile doublelithiation of a transient urea: vicarious ortho-metalation of aniline derivatives. Org Lett, 2010. 12(13): p. 3090-2.

204. Fadok, V.A., et al., Exposure of phosphatidylserine on the surface of apoptotic lymphocytes triggers specific recognition and removal by macrophages. $\mathrm{J}$ Immunol, 1992. 148(7): p. 2207-16.

205. Ghosh, M.C., et al., Insulin-like growth factor-I stimulates differentiation of ATII cells to ATI-like cells through activation of Wnt5a. Am J Physiol Lung Cell Mol Physiol, 2013. 305(3): p. L222-8.

206. Cheranov, S.Y., et al., An essential role for SRC-activated STAT-3 in 14,15-EETinduced VEGF expression and angiogenesis. Blood, 2008. 111(12): p. 5581-91.

207. Pi, M., et al., Structural and Functional Evidence for Testosterone Activation of GPRC6A in Peripheral Tissues. Mol Endocrinol, 2015. 29(12): p. 1759-73.

208. FDA, FDA's policy statement for the development of new stereoisomeric drugs. Chirality, 1992. 4(5): p. 338-40. 
209. Ericsson, U.B., et al., Thermofluor-based high-throughput stability optimization of proteins for structural studies. Analytical Biochemistry, 2006. 357(2): p. 289298.

210. Schaller, M.D., et al., Autophosphorylation of the focal adhesion kinase, pp125FAK, directs SH2-dependent binding of pp60src. Mol Cell Biol, 1994. 14(3): p. 1680-8.

211. Blake, R.A., et al., SU6656, a selective src family kinase inhibitor, used to probe growth factor signaling. Mol Cell Biol, 2000. 20(23): p. 9018-27.

212. Akagi, T., et al., v-Crk activates the phosphoinositide 3-kinase/AKT pathway by utilizing focal adhesion kinase and H-Ras. Mol Cell Biol, 2002. 22(20): p. 701523.

213. Fujikawa, K., et al., Role of PI 3-kinase in angiopoietin-1-mediated migration and attachment-dependent survival of endothelial cells. Exp Cell Res, 1999. 253(2): p. 663-72.

214. Du, J., et al., PI3K and ERK-induced Racl activation mediates hypoxia-induced HIF-1alpha expression in MCF-7 breast cancer cells. PLoS One, 2011. 6(9): $\mathrm{p}$. e25213.

215. Yoshida, A., B. Anand-Apte, and B.R. Zetter, Differential endothelial migration and proliferation to basic fibroblast growth factor and vascular endothelial growth factor. Growth Factors, 1996. 13(1-2): p. 57-64.

216. Hoellerer, M.K., et al., Molecular recognition of paxillin LD motifs by the focal adhesion targeting domain. Structure, 2003. 11(10): p. 1207-17.

217. Gao, G., et al., NMR solution structure of the focal adhesion targeting domain of focal adhesion kinase in complex with a paxillin LD peptide: evidence for a twosite binding model. J Biol Chem, 2004. 279(9): p. 8441-51.

218. Birukova, A.A., et al., Paxillin is involved in the differential regulation of endothelial barrier by HGF and VEGF. Am J Respir Cell Mol Biol, 2009. 40(1): p. 99-107.

219. Yang, W.J., et al., Paxillin regulates vascular endothelial growth factor Ainduced in vitro angiogenesis of human umbilical vein endothelial cells. Mol Med Rep, 2015. 11(3): p. 1784-92.

220. Schaller, M.D., Paxillin: a focal adhesion-associated adaptor protein. Oncogene, 2001. 20(44): p. 6459-72.

221. Iwasaki, T., et al., Involvement of phosphorylation of Tyr-31 and Tyr-118 of paxillin in MMI cancer cell migration. Int J Cancer, 2002. 97(3): p. 330-5.

222. Kadare, G., et al., Conformational dynamics of the focal adhesion targeting domain control specific functions of focal adhesion kinase in cells. J Biol Chem, 2015. 290(1): p. 478-91.

223. Subauste, M.C., et al., Vinculin modulation of paxillin-FAK interactions regulates ERK to control survival and motility. J Cell Biol, 2004. 165(3): p. 371-81.

224. Yan, W., B. Bentley, and R. Shao, Distinct angiogenic mediators are required for basic fibroblast growth factor-and vascular endothelial growth factor-induced angiogenesis: the role of cytoplasmic tyrosine kinase c-Abl in tumor angiogenesis. Mol Biol Cell, 2008. 19(5): p. 2278-88. 
225. Pylayeva, Y., et al., Ras- and PI3K-dependent breast tumorigenesis in mice and humans requires focal adhesion kinase signaling. J Clin Invest, 2009. 119(2): p. 252-66.

226. Shen, T.L. and J.L. Guan, Differential regulation of cell migration and cell cycle progression by FAK complexes with Src, PI3K, Grb7 and Grb2 in focal contacts. FEBS Lett, 2001. 499(1-2): p. 176-81.

227. Tang, J., et al., Vascular endothelial growth factor promotes cardiac stem cell migration via the PI3K/Akt pathway. Exp Cell Res, 2009. 315(20): p. 3521-31.

228. Bullard, L.E., X. Qi, and J.S. Penn, Role for extracellular signal-responsive kinase-1 and -2 in retinal angiogenesis. Invest Ophthalmol Vis Sci, 2003. 44(4): p. 1722-31.

229. Thamilselvan, V., D.H. Craig, and M.D. Basson, FAK association with multiple signal proteins mediates pressure-induced colon cancer cell adhesion via a Srcdependent PI3K/Akt pathway. FASEB J, 2007. 21(8): p. 1730-41.

230. Thakker, G.D., et al., The role of phosphatidylinositol 3-kinase in vascular endothelial growth factor signaling. J Biol Chem, 1999. 274(15): p. 10002-7.

231. Bertolucci, C.M., C.D. Guibao, and J. Zheng, Structural features of the focal adhesion kinase-paxillin complex give insight into the dynamics of focal adhesion assembly. Protein Sci, 2005. 14(3): p. 644-52.

232. Hayashi, I., K. Vuori, and R.C. Liddington, The focal adhesion targeting (FAT) region of focal adhesion kinase is a four-helix bundle that binds paxillin. Nat Struct Biol, 2002. 9(2): p. 101-6.

233. Zhang, Z.M., et al., GIT1 paxillin-binding domain is a four-helix bundle, and it binds to both paxillin LD2 and LD4 motifs. J Biol Chem, 2008. 283(27): $\mathrm{p}$. 18685-93.

234. Gilmore, A.P. and L.H. Romer, Inhibition of focal adhesion kinase (FAK) signaling in focal adhesions decreases cell motility and proliferation. Mol Biol Cell, 1996. 7(8): p. 1209-24.

235. Gogate, P.N., et al., Design, synthesis, and biological evaluation of novel FAK scaffold inhibitors targeting the FAK-VEGFR 3 protein-protein interaction. Eur J Med Chem, 2014. 80: p. 154-66.

236. Beaumont, K.G. and M. Mrksich, The mechanostability of isolated focal adhesions is strongly dependent on $\mathrm{pH}$. Chem Biol, 2012. 19(6): p. 711-20.

237. Cable, J., et al., In vitro phosphorylation of the focal adhesion targeting domain of focal adhesion kinase by Src kinase. Biochemistry, 2012. 51(11): p. 2213-23.

238. Werdich, X.Q. and J.S. Penn, Specific involvement of SRC family kinase activation in the pathogenesis of retinal neovascularization. Invest Ophthalmol Vis Sci, 2006. 47(11): p. 5047-56.

239. Bautch, V.L. and J.M. James, Neurovascular development: The beginning of a beautiful friendship. Cell Adh Migr, 2009. 3(2): p. 199-204.

240. Lee, B.Y., et al., FAK signaling in human cancer as a target for therapeutics. Pharmacol Ther, 2015. 146: p. 132-49.

241. Ischenko, I., et al., Effect of Src kinase inhibition on metastasis and tumor angiogenesis in human pancreatic cancer. Angiogenesis, 2007. 10(3): p. 167-82.

242. Sweeney, W.E., Jr., et al., Src inhibition ameliorates polycystic kidney disease. J Am Soc Nephrol, 2008. 19(7): p. 1331-41. 
243. Taylor, J.W., et al., Phase 2 study of bosutinib, a Src inhibitor, in adults with recurrent glioblastoma. J Neurooncol, 2015. 121(3): p. 557-63.

244. Sulzmaier, F.J., C. Jean, and D.D. Schlaepfer, FAK in cancer: mechanistic findings and clinical applications. Nat Rev Cancer, 2014. 14(9): p. 598-610.

245. Jagadeeswaran, R., et al., Paxillin is a target for somatic mutations in lung cancer: implications for cell growth and invasion. Cancer Res, 2008. 68(1): p. 132-42.

246. Pascolini, D. and S.P. Mariotti, Global estimates of visual impairment: 2010 . Br J Ophthalmol, 2012. 96(5): p. 614-8.

247. Klein, R., et al., The 25-year incidence of visual impairment in type 1 diabetes mellitus the wisconsin epidemiologic study of diabetic retinopathy. Ophthalmology, 2010. 117(1): p. 63-70.

248. Klein, R., et al., Markers of inflammation, oxidative stress, and endothelial dysfunction and the 20-year cumulative incidence of early age-related macular degeneration: the Beaver Dam Eye Study. JAMA Ophthalmol, 2014. 132(4): p. 446-55.

249. Alon, T., et al., Vascular endothelial growth factor acts as a survival factor for newly formed retinal vessels and has implications for retinopathy of prematurity. Nat Med, 1995. 1(10): p. 1024-8.

250. Pierce, E.A., et al., Vascular endothelial growth factor/vascular permeability factor expression in a mouse model of retinal neovascularization. Proc Natl Acad Sci U S A, 1995. 92(3): p. 905-9.

251. Aiello, L.P., et al., Suppression of retinal neovascularization in vivo by inhibition of vascular endothelial growth factor (VEGF) using soluble VEGF-receptor chimeric proteins. Proc Natl Acad Sci U S A, 1995. 92(23): p. 10457-61.

252. Matuszewski, B.K., M.L. Constanzer, and C.M. Chavez-Eng, Strategies for the assessment of matrix effect in quantitative bioanalytical methods based on HPLCMS/MS. Anal Chem, 2003. 75(13): p. 3019-30.

253. Ramagiri, S., et al., Fast and sensitive liquid chromatography/electrospray mass spectrometry method to study ocular penetration of EDL-155, a novel antitumor agent for retinoblastoma in rats. J Mass Spectrom, 2009. 44(5): p. 786-93.

254. Song, P., et al., Plasma and cerebrospinal fluid pharmacokinetics of the novel tetrahydroisoquinoline EDL-155 in rats. Cancer Chemother Pharmacol, 2008. 61(6): p. 1037-44.

255. Bamborough, P., et al., Assessment of chemical coverage of kinome space and its implications for kinase drug discovery. J Med Chem, 2008. 51(24): p. 7898-914.

256. Mikacic, I. and D. Bosnar, Intravitreal Bevacizumab and Cardiovascular Risk in Patients with Age-Related Macular Degeneration: Systematic Review and MetaAnalysis of Randomized Controlled Trials and Observational Studies. Drug Saf, 2016.

257. Yu, J., et al., Anti-VEGF Therapy with Bevacizumab--limited cardiovascular toxicity. Asian Pac J Cancer Prev, 2014. 15(24): p. 10769-72.

258. Song, P., et al., Plasma and cerebrospinal fluid pharmacokinetics of the novel tetrahydroisoquinoline EDL-155 in rats. Cancer Chemother Pharmacol, 2007.

259. Schuster, D., C. Laggner, and T. Langer, Why drugs fail--a study on side effects in new chemical entities. Curr Pharm Des, 2005. 11(27): p. 3545-59. 
260. de Bodinat, C., et al., Agomelatine, the first melatonergic antidepressant: discovery, characterization and development. Nat Rev Drug Discov, 2010. 9(8): p. 628-42.

261. Ferracioli-Oda, E., A. Qawasmi, and M.H. Bloch, Meta-analysis: melatonin for the treatment of primary sleep disorders. PLoS One, 2013. 8(5): p. e63773.

262. Choueiri, T.K., et al., Congestive heart failure risk in patients with breast cancer treated with bevacizumab. J Clin Oncol, 2011. 29(6): p. 632-8. 


\section{VITA}

Jordan Javad Toutounchian was born in 1985 in Plano, Texas, USA. In 2007, he received his Bachelors of Science degree from the University of Texas at Austin with a major focus in neurobiology. In 2010, he began his graduate studies under the tutelage of Dr. Ryan Yates, professor in the Department of Pharmaceutical Sciences at the University of Tennessee Health Science Center. He received the Doctor of Philosophy degree in Pharmaceutical Sciences in the summer of 2016. 